\title{
DeVElopment of Th2 IMMUNE RESPONSE MODELS TO EVALUATE ALLERGENICITY OF MILK PROTEINS
}

by

Marcus James Robinson

A thesis submitted to the Victoria University of Wellington in fulfilment of the requirements for the degree of Doctor of Philosophy

Victoria University of Wellington 2013 


\section{Abstract}

Food allergy, defined as an adverse immune response to food, is increasing in prevalence. It can be broadly separated into phases of sensitization, in which allergytriggering Immunoglobulin $\mathrm{E}(\mathrm{IgE})$ is generated, and the post-sensitization allergic response, in which the allergic response is triggered by sensitizing allergen. While much is known about the specific mediators that cause allergies, the immune processes that underlie disease progression are less clear. This project has employed mouse models of Th2 immunity to clarify the factors involved in the initiation and maintenance of allergic disease.

At the centre of allergic disease is the Interleukin (IL)-4-producing CD4+ T helper type 2 (Th2) cell. One of the key inducers of Th2 cell development in vitro is IL-4, but its involvement in Th2 cell development in vivo is controversial. In our studies, we saw that Th2 cell development could be initiated in vivo by primary, adjuvant-free allergen immunisation in the absence of IL-4. However, Th2 cells were more frequent in IL-4-sufficient conditions. We also determined that genetic lesions that result in loss of one, or both, IL-4 alleles impaired the Th2 cell-mediated allergic process, such that IL-4-heterozygous mice can be considered haplo-insufficient for IL-4 in allergic disease contexts.

In addition to the generation of $\operatorname{IgE}$ antibody, Th2 cells are implicated in the postsensitization phase of allergy. Multiple oral challenges of sensitized mice induces elevations in Th2-associated cytokines and elevates intestinal mast cell frequencies. It was the second aim of this project to clarify the role of CD4+ $\mathrm{T}$ cells in the post- 
sensitization intestinal allergic process. We demonstrate a key role for CD4+ T cells in this jejunal mast cell recruitment, and identify that this is required in addition to their established contribution to IgE production. Our investigations also reveal a previously unappreciated role for the CD4+ T cell-derived cytokine IL-3 in oral food allergy. These findings suggest that intestinally localised mast cell-inducer Th2 $(\mathrm{Th} 2 \mathrm{~m})$ cells are required for allergic responses generated in the intestine.

We also investigated whether specific components of ruminant milks influence the allergic process. While goat and cow milks share significant protein homology, goat milk has lower sensitizing and response-evoking capacity, or allergenicity, than cow milk, in numerous experimental systems. In this project, we compared dominant allergens purified from cow and goat milks for their ability to initiate Th2 cell development. We also examined the ability of one of these allergens to initiate the intestinal allergic process. In these studies, we observed similar Th2 cell development and intestinal mast cell activity in response to both cow and goat milk proteins. These responses indicate that the intrinsic allergenicity of the proteins analysed is not sufficient to explain the differential allergenicity attributed to cow and goat milk.

These studies examine the endogenous and exogenous factors that contribute to the development of allergic disease. This project clarifies the role of IL-4 in in vivo Th2 cell development, identifies functional segregation of CD4+ Th2 cells in the intestinal allergic process and further illustrates some of the similarities in the allergenicity of isolated cow and goat milk proteins. Collectively, these studies uncover fundamental aspects of the allergic process which may be useful targets for disease intervention in both prophylactic and therapeutic settings. 


\section{Acknowledgements}

Graham, thank you for challenging me intellectually and helping me grow. Thank you for teaching me how to ask questions that can be tested quantitatively. Thank you for your optimism and enthusiasm. I know I have learned a lot, but more than anything I am glad to have learned how to think beyond the data that I see. It has been a privelege to study at the institute you, Franca, your board and colleagues have built over the last two decades.

Ali, thank you for all of your advice, visits and input. Thanks for giving me the time to learn about allergic immunity as I grappled with the features that make an allergen an allergen. Thanks for your help with proofing. Thank you for giving me the purified milk proteins and getting more purified when I requested them as well.

Liz, thank you for your stalwart support, for discussing ideas and bandying about some great hypotheses. I admire your altruism and dedication to your research. Thanks for being a good 'elder lab cousin'.

Sabine, Janice, Lindsay and Rene thanks for your help and advice during the write-up process. Thanks for your tips on style, structure and type-setting, too.

Ryan, Dave, Cat, Helen, and Sou, thank you for being a sounding board for some of my crazy concepts. It was really fun discussing ideas with you, even late at night.

Mali, thanks for teaching me about worms and for being so patient whenever I had 'a few' extra mice in stock. Similarly Mel, thanks for taking over a couple of poorly explained but well executed experiments. The two of you have magic hands and I 
thank you two, Mark, Evelyn, Fen and Api for always saying good morning with a smile. Jess and Shiau-Choot, thanks for organising things and keeping the lab in order, always.

Jane, thank you for doing the sectioning with a smile. Ariane, Marie and Irene, thanks for your encouragement.

To the other PhD club members: thanks for discussing projects and being so continually positive that it had to rub off eventually. Thanks, too, to those of you who have shared advice on techniques. To the Malaghan support staff: thanks for making things run smoothly. Thank you BRU team for looking after my mice.

To the collective group leaders at the Malaghan: Thank you for supporting students and maintaining an open environment for discussion. Thanks too for bearing with me, because you have all helped me at some point. Jacquie, thanks for asking how things are going from time-to-time too.

Anne La Flamme and Darren Day, thanks for encouraging me to apply for a $\mathrm{PhD}$ position.

Rebecca, you are beautiful, amazing and I love you. Thanks for standing by me, for making me laugh and encouraging me everyday. Thanks for helping me realise I can't be anyone but myself and encouraging me to be a better version of myself. I'm glad we get to walk together. Thanks for learning some key terms, like 'flow cytometer' and 'pipette' too!

God, thanks for showing me great things in the big and the small. Omniscience would be nice, so thanks for giving us the tools to make small steps towards understanding everyday. Sometimes it seems the finest technologies we have are equivalent to using 
a brick as a key, but still the answers seem to come. I'm reminded, of course, of Ecclesiastes 12:12, and of Psalm 127. I hope that you will help us find the answer soon.

Lastly, to the funding body, formerly MSI, formerly FoRST. Thank you for providing my supervisors with funding for this project. They have spent significant time developing me as a scientist and while the most important findings have not yet provided New Zealanders with a hypoallergenic food product, the questions addressed have made significant progress towards this goal. 


\section{Disclosure statement}

Histological sectioning and chloroacetate esterase staining were performed by Jane Anderson of the Wellington School of Medical Research. Elizabeth Forbes-Blom, Catherine Plunkett, Ryan Kyle and Mali Camberis have assisted with tissue isolation and processing in some CD4 depletion experiments. Elizabeth Forbes-Blom performed some intragastric challenges; Catherine Plunkett and Ian Saldahna acted as blinded observers in some cases of diarrhoea monitoring and helped with some photography. Milk proteins were purifed by Ali Hodgkinson at AgResearch Ruakura. The primary Th2 model described in the introduction was conceived and developed by Graham Le Gros, Mali Camberis and Melanie Prout. Differential allergenicity of dead $i$ L3 and HDM were first observed by them. One intradermal assay and one oral allergeninduced diarrhoea assay were undertaken by Melanie Prout. With Graham Le Gros, Helen Mearns originally conceived the studies examining the biallelic expression profile of IL-4 in vivo, from which the investigations in the KN2/G4 reporter system began. With these disclosures, I declare that the content of this document is my own work.

Marcus Robinson, 2012. 


\section{Table of Contents}

Abstract vii

Acknowledgements vii

Disclosure statement vii

Table of Contents vii

List of Figures xiii

Abbreviations xiii

1 General Introduction 1

1.1 Thesis overview . . . . . . . . . . . . . . . . . . . 1

1.2 Allergy and food induced allergic reactions . . . . . . . . . . . . . 3

1.2.1 What is allergy? . . . . . . . . . . . . 3

1.2.2 $\operatorname{IgE}$ is at the centre of allergic disease . . . . . . . . . . 3

1.2.3 Physiological changes that occur during systemic anaphylaxis . 5

1.2.4 Physiological processes during fasting and allergic intestinal responses ........................ 6

1.2.5 IgE and mast cells mediate intestinal allergen-induced allergic responses . . . . . . . . . . . . . . . . . 7

1.2.6 Management of food allergy . . . . . . . . . . . . . . 11

1.2.7 Identification of food allergy . . . . . . . . . . . . . . . . . 12

1.2.8 Hereditary risk factors for food allergy . . . . . . . . . . . . . 12

1.2.9 Diet and the intestinal microbiota influence disease risk . . . . . 13

1.2.10 Environmental exposure as a risk factor for allergy . . . . . . . 14

1.2 .11 Oral tolerance . . . . . . . . . . . . . . . . . 15

1.3 Allergenicity and the epidemiology of allergic disease . . . . . . . . . . 18

1.3.1 Allergy and allergenicity . . . . . . . . . . . . . . 18

1.3.2 Molecular properties of allergens, with specific reference to food allergens . . . . . . . . . . . . . . . . . . . . 19

1.3.3 The epidemiology of food allergy . . . . . . . . . . . . 21

1.3.4 Sensitization to multiple foods . . . . . . . . . . . . . 22

1.3.5 Atypical mechanisms of sensitisation . . . . . . . . . . . 23

1.3.6 Physical and immunological differences in cow and goat milks . 24

1.4 Predicting allergenicity . . . . . . . . . . . . . . 28 
1.4.1 In silico prediction . . . . . . . . . . . . . . . . . 28

1.4.2 Biological evaluation of allergenicity and cross-reactivity . . . . 29

1.4.3 Development of a novel assay of allergenicity and Th2 function . 31

1.5 The immunological mechanisms of allergic disease . . . . . . . . . . . 35

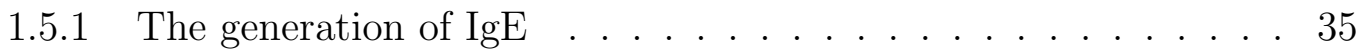

1.5.2 Additional roles of IL-4 . . . . . . . . . . . . . . . . . 36

1.5.3 Mice are rendered susceptible to intestinally-triggered allergic responses in a post-sensitization oral potentiation phase . . . . 36

1.5.4 CD4+ T cells are implicated in oral potentiation . . . . . . . . . 37

1.5.5 Cytokine requirements for intestinal mastocytosis . . . . . . . . 38

1.5.6 Intestinal mastocytosis heightens allergic disease susceptibility . 39

1.6 Th2 cells and the functional Th paradigm . . . . . . . . . . . . . . . 41

1.6.1 The early and late events specifying Th2 polarization in experimental systems . . . . . . . . . . . . . . . . . . . 42

1.6.2 Are there multiple populations of IL-4-producing CD4+ T cells? 44

1.6.3 Measuring IL-4 production using IL-4 reporter mice . . . . . . . 46

1.6.4 The regulation of IL-4 production . . . . . . . . . . . . . . . . 49

1.7 Themes of this thesis . . . . . . . . . . . . . . . . . 53

2 Methods $\quad 55$

2.1 Special reagents and miscellaneous procedures . . . . . . . . . . 55

2.1.1 Allergens . . . . . . . . . . . . . . . . . . 55

2.1 .2 Mice . . . . . . . . . . . . . . . . 56

2.1.3 Genotyping . . . . . . . . . . . . . 57

2.1.4 Protein quantification . . . . . . . . . . . . . . 58

2.1.5 Trypan blue exclusion test . . . . . . . . . . . . . . . . . 58

2.1.6 Prediction of sequence homology using DELTA BLAST . . . . . 58

2.1.7 Statistical analysis and graphics . . . . . . . . . . . . . . 59

2.2 Cell culture . . . . . . . . . . . . . . . . . . . . . . 60

2.2.1 Hybridoma culture . . . . . . . . . . . . . . . . . . 60

2.2.2 Bone marrow cell culture for IL-3- and TSLP-elicited basophils . 61

2.2.3 Th cell polarization . . . . . . . . . . . . . . . . . 61

2.2.4 In vitro restimulation of Th cells . . . . . . . . . . . . . 62

2.2.5 IgG1 spot assay . . . . . . . . . . . . . . . . . . . . 63 
2.3 Mouse manipulations . . . . . . . . . . . . . . . . . . . 63

2.3.1 Primary Th2 (pTh2) assay . . . . . . . . . . . . . 63

2.3.2 Oral allergen-induced experimental anaphylaxis (diarrhoea) model 64

2.3.3 Active systemic anaphylaxis . . . . . . . . . . . . . . . 65

2.3.4 Packed Cell Volume (PCV) _ . . . . . . . . . . . . . 65

2.3.5 Serum isolation . . . . . . . . . . . . . . . . 65

2.3 .6 Tissue isolation . . . . . . . . . . . . . . 65

2.3.7 Processing of jejunal tissue . . . . . . . . . . . . . . 66 66

2.3 .8 Bone marrow preparation . . . . . . . . . . . . 66

2.3 .9 Splenocytes . . . . . . . . . . . . . . . . . . 67

2.3 .10 Lymph node cells . . . . . . . . . . . . . . . . . . . . 67

2.3 .11 Jejunal mast cell quantification . . . . . . . . . . . . 6 67

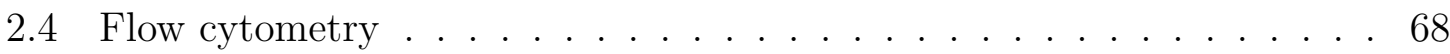

2.4.1 Flow Cytometry reagents . . . . . . . . . . . . . . 68

2.4 .2 Surface staining . . . . . . . . . . . . . . . . . . . 69

2.4 .3 Intracellular staining . . . . . . . . . . . . . . . . . . . 69

2.4 .4 Flow cytometers . . . . . . . . . . . . . . . . 69

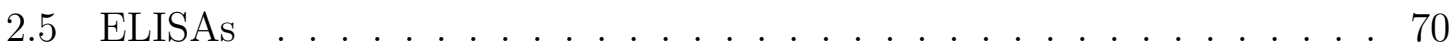

2.5.1 Antigen-specific IgG1, IgG2a and polyclonal Ig ELISA $\ldots . .70$

$2.5 .2 \quad \operatorname{IgE} \ldots \ldots \ldots \ldots \ldots \ldots \ldots$

2.5.3 Murine mast cell protease-1 (mMCP-1) . . . . . . . . . . . 71

2.5.4 Total IgG1 and Total IgG2a . . . . . . . . . . . . . . . . 72

3 Does the in vitro IL-4 allelic expression profile reflect functional segregation of IL-4-producing Th2 cell subsets?

3.1 Introduction . . . . . . . . . . . . . . . . . . 73

3.1.1 Monoallelic regulation of the IL-4 gene . . . . . . . . . 73

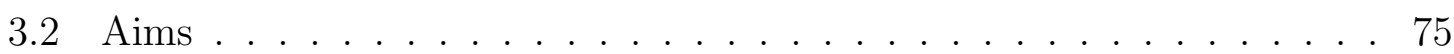

3.3 Results . . . . . . . . . . . . . . . . . . 76

3.3.1 Examining IL-4 reporter expression during primary culture . . . 76

3.3.2 Examining IL-4 production in differentiated Th2 cells . . . . . 80

3.3.3 Testing for interactions between IL-4, KN2 and G4 . . . . . 84

3.3.4 Comparison of IL-4 production in homozygous and heterozy-

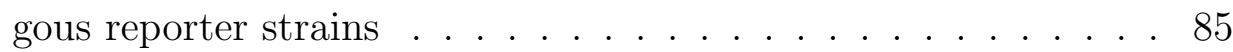


3.4 Discussion . . . . . . . . . . . . . . . . . . . . . . . . . 91

3.4.1 Probabilistic monoallelic regulation of IL-4 . . . . . . . . . . . . 91

3.5 Summary . . . . . . . . . . . . . . . . . . . . . . . 98

4 Evaluating the contribution of IL-4 to Th2 cell development and function in vivo $\quad 99$

4.1 Introduction . . . . . . . . . . . . . . . . . . . . . . 99

4.1.1 Establishing how genetic lesions in IL-4 affect allergic Th2 cell development and function in vivo . . . . . . . . . . . . . . . . . . 99

4.2 Aims . . . . . . . . . . . . . . . . . . . 101

4.3 Results . . . . . . . . . . . . . . . . . . . . 102

4.3.1 Testing whether IL-4 is required for in vivo Th2 cell development102

4.3.2 Evaluating the influence of IL-4 on primary, adjuvant-free in vivo Th2 cell development . . . . . . . . . . . . . . . . . . . . 104

4.3.3 Establishing the role of STAT-6 in primary, adjuvant-free in vivo Th2 cell development . . . . . . . . . . . . . . . . . . . 109

4.3.4 Examining the influence of IL-4 on Th2-dependent immune responses . . . . . . . . . . . . . . . . . 111

4.3.5 The influence of a single IL-4 allele on primary, adjuvant-free IgG1-class switch recombination . . . . . . . . . . . . . . . 122

4.3.6 The influence of IL-4 heterozygosity on the intestinal allergic response . . . . . . . . . . . . . . . . . 126

4.4 Discussion . . . . . . . . . . . . . . . . . . . . . . 131

4.4.1 The role of IL-4 in Th2 cell development . . . . . . . . . . . . . 131

4.4.2 Potential mechanisms that promote IL-4-independent Th2 cell development in vivo . . . . . . . . . . . . . . . . . . . . . . 134

4.4.3 Potential implications on IL-4-dependent Th2 cell expansion . . 135

4.4.4 A glimpse at the allelic expression of IL-4 in vivo . . . . . . . . 140

4.4.5 Modulating IL-4 levels in Th2 immunity . . . . . . . . . . . . . 141

4.4.6 IL-4 haplo-insufficiency in Th2 immunity . . . . . . . . . . . . . 142

4.4.7 Mechanisms that may underlie the haplo-insufficient phenotype of IL-4-heterozygous mice . . . . . . . . . . . . . . . . . . . 147

4.4.8 The effect of IL-4 heterozygosity on the intestinal allergic process 148

4.5 Summary . . . . . . . . . . . . . . . . . . . . . . 149 
5 Establishing an allergy-permissive environment through allergen exposure in the gut

5.1 Introduction . . . . . . . . . . . . . . . . . . . . . . 151

5.1.1 Assessing the role of CD4 $\mathrm{T}$ cells in the post-sensitization phase of food allergy . . . . . . . . . . . . . . 151

5.2 Aims . . . . . . . . . . . . . . . . . . . . . 153

5.3 Results . . . . . . . . . . . . . . . . . . . 154

5.3.1 The role of CD4+ T cells in oral potentiation . . . . . . . . . 154

5.3.2 Segregating CD4+ T cell-dependent mastocytosis and IgE pro-

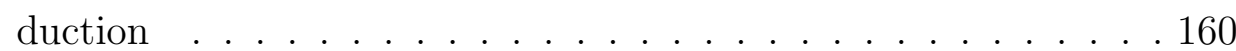

5.3.3 Examining the role of CD4+ T cells in disease maintenance . . 162

5.3.4 The role of CD4+ $\mathrm{T}$ cells in systemic responses after oral po-

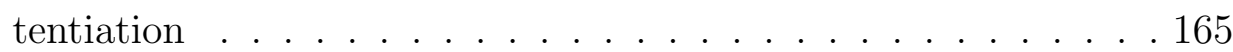

5.3.5 Influence of CD4+ T cells on plasma volume loss . . . . . . . 167

5.3.6 The cytokines that influence allergic mastocytosis . . . . . . . . 169

5.3.7 The relative influence of IL-3 and IL-9 on allergic mastocytosis . 176

5.3.8 Assessing the role of IL-3 in i.v.-induced intestinal allergy . . . . 181

5.4 Discussion . . . . . . . . . . . . . . . . . . . . . . . . . . . 184

5.4.1 The role of CD4+ T cells in oral potentiation . . . . . . . . . 184

5.4.2 Distinct roles for CD4+ T cells in intestinal mastocytosis and $\mathrm{IgE}$ generation . . . . . . . . . . . . . . . . . 185

5.4.3 The role of CD4+ T cells in disease maintenance . . . . . . . . 186

5.4.4 Clarifying the earliest events in the intiation of oral potentiation 188

5.4.5 Requirement for oral potentiation for diarrhoea in different contexts . . . . . . . . . . . . . . . . . 190

5.4.6 The role of IL-3 in mast cell-dependent Th2 immunity . . . . . 191

5.4 .7 The role of IL-3 in intestinal food allergy . . . . . . . . . . . . . 192

5.4.8 The source of IL-3 that drives allergic intestinal mastocytosis . . 193

5.4.9 Additional potential effects of IL-3 on intestinal mast cell function194

5.5 Summary . . . . . . . . . . . . . . . . . . . 194

6 Examining milk allergenicity in Th2 immune response models 195

6.1 Introduction . . . . . . . . . . . . . . . . . . . . . . . 195

6.1.1 Rationale for examining goat milk allergenicity . . . . . . . . 195 
6.2 Aims . . . . . . . . . . . . . . . . . . . . 197

6.3 Results . . . . . . . . . . . . . . . . . . . . . . 198

6.3.1 Examining pTh2 responses to $\beta$-lactoglobulin . . . . . . . . 198

6.3.2 Examining pTh2 responses to casein proteins. . . . . . . . . . 199

6.3.3 Examining the ability of goat milk to suppress Th2 responses . 207

6.3.4 The oral response to milk allergens . . . . . . . . . . . . . . 209

6.4 Discussion . . . . . . . . . . . . . . . . . . . . . . . 215

6.4.1 Evidence for differential allergenicity of goat and cow milk . . . 215

$6.4 .2 \alpha$ s1-casein as an allergen . . . . . . . . . . . . . . 216

6.4.3 The allergenicity of BLG . . . . . . . . . . . . . . . 218

6.4.4 Future investigations that could clarify goat milk protein allergenicity . . . . . . . . . . . . . . . . . 219

6.5 Summary . . . . . . . . . . . . . . . . . . . 220

7 General Discussion $\quad 221$

7.1 Summary of findings . . . . . . . . . . . . . . . . . . . . . 221

7.1.1 Checkpoints along the road to allergy . . . . . . . . . . . 221

7.1 .2 Future research directions . . . . . . . . . . . . . . . . . 223

7.1.3 Evaluating the success of this project . . . . . . . . . . . . 227

7.1.4 Final conclusions . . . . . . . . . . . . . . . . . 227

8 Appendices $\quad 278$

8.1 Supporting data . . . . . . . . . . . . . . . . . . . . . 278

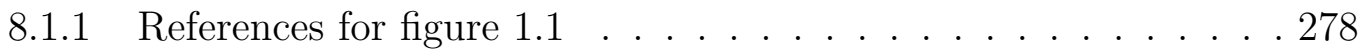

8.1.2 Genotyping of IL-3 and IL-9 transgene . . . . . . . . . . . . . . 279

8.1 .3 Flow Cytometry reagents . . . . . . . . . . . . . . . . 280

8.1 .4 Flow cytometers . . . . . . . . . . . . . . . . . 282

8.1.5 Supplementary data . . . . . . . . . . . . . . . . 284 


\section{List of Figures}

General Introduction $\quad 1$

1.1 Physiological changes induced by major mediators of anaphylaxis. . . . 10

1.2 The immune response is constrained to a single lymph node. . . . . . . 32

1.3 Hierarchical clustering of allergens by Th2 cell development. . . . . . . 33

1.4 Sensitive and consistent Th2 response to parasite and dust mite allergens. 34

1.5 Insertion sites of 4 get, KN2 and G4 reporter constructs. . . . . . . . . . 48

1.6 Probabilistic and deterministic mechanisms of gene regulation . . . . . 52

Does the in vitro IL-4 allelic expression profile reflect functional $\begin{array}{ll}\text { segregation of IL-4-producing Th2 cell subsets? } & \mathbf{7 3}\end{array}$

$3.1 \mathrm{KN} 2 / \mathrm{G} 4$ cells cultured with IL-4 express IL-4 reporters. . . . . . . . . . 78

3.2 Culture in IL-4 increases biallelic IL-4 production above predicted levels. 79

3.3 Culture in IL-4 potentiates cells to produce IL-4 biallelically upon secondary stimulation. . . . . . . . . . . . . . . . . . 82

3.4 Biallelic IL-4 production exceeds predicted frequencies in restimulated $\mathrm{KN} 2 /+$ and $\mathrm{G} 4 /+$ cells. . . . . . . . . . . . . . . . . 83

3.5 IL-4 production is equivalent in heterozygous reporter cells. . . . . . . . 87

3.6 Reporter expression by homozygous and heterozygous reporter cells cultured in anti-IL-4. . . . . . . . . . . . . . . . . 88

3.7 Reporter expression by homozygous and heterozygous reporter cells cultured in IL-4. . . . . . . . . . . . . . . . . . . . . . 89

3.8 hCD2 and GFP behave similarly in the absence of IL-4 signalling. . . . 90

3.9 Probabilistic regulation may provide tighter regulation of IL-4-dependent responses . . . . . . . . . . . . . . . . . . 997

Evaluating the contribution of IL-4 to Th2 cell development and function in vivo $\quad 99$

4.1 Th2 cells develop in the absence of IL-4 in vivo. . . . . . . . . . . . . 103

4.2 IL-4-independent and dependent phases of Th2 development revealed in IL-4 reporter mice. . . . . . . . . . . . . . . . . . . . . . 106 
4.3 IL-4-independent and dependent phases of Th2 development confirmed in alternate reporter mice. . . . . . . . . . . . . . . . . . . 107

4.4 IL-4 enhances Th2 response to parasite allergens. . . . . . . . . . . . . 108

4.5 IL-4-dependent Th2 response requires STAT-6. . . . . . . . . . . . . . . 110

4.6 Th2 cells exhibit an activated TFH cell phenotype. . . . . . . . . . . . 113

4.7 IL-4 production is common in TFH cells. . . . . . . . . . . . . . . . . . 114

4.8 IL-4 can derive from one or both alleles in TFH cells. . . . . . . . . . . 116

4.9 IL-4 is produced by GL-7+ (germinal centre) TFH cells. . . . . . . . . 118

$4.10 \mathrm{TFH}$ cell function is reported by IgG1+ B cell development. . . . . . . 120

4.11 IgG1 class-switch B cell response is antigen-specific. . . . . . . . . . . . 121

4.12 IgG1 class-switch B cell response requires STAT-6. . . . . . . . . . . . 123

4.13 The IgG1 class-switch response is IL-4 allele dose-dependent. . . . . . . . 125

$4.14 \mathrm{IgE}$ production is impaired in IL-4 heterozygous mice. . . . . . . . . . . 128

4.15 $\mathrm{IgE}$ production is impaired in hyper-primed, orally-challenged, IL-4 heterozygous mice. . . . . . . . . . . . . . . . . . . . . . . . . 129

4.16 IL-4 heterozygosity prevents oral allergen-induced intestinal anaphylaxis.130 4.17 Potential mechanisms of IL-4-dependent Th2 cell expansion. . . . . . . 139

\section{Establishing an allergy-permissive environment through allergen} exposure in the gut

5.1 CD4+ T cell depletion prevented diarrhoea and oral potentiation. . . . 156

5.2 CD4+ T cell depletion did not prevent systemic anaphylaxis. . . . . . . 157

5.3 Diarrhoea development correlated with the re-emergence of CD4+ $\mathrm{T}$ cells. . . . . . . . . . . . . . . . . . . . . 159

5.4 High-titre IgE can only confer disease susceptiblity in the presence of CD4+ T cells. . . . . . . . . . . . . . . . . . . . . . 161

5.5 Orally primed mice exhibited diarrhoea even in the absence of CD4+ T cells. . . . . . . . . . . . . . . . . . . . . 163

5.6 CD4+ T cell depletion diminished the Th2-associated antibody response. 164

5.7 Orally-primed mice developed i.v.-induced anaphylaxis with or without CD4+ T cells. . . . . . . . . . . . . . . . . . . . . . 166

5.8 Haematocrit increases in intestinally anaphylactic mice with or without CD4+ T cells. . . . . . . . . . . . . . . . . . . . . 168 
5.9 Th2 cells produced more IL-3 than Th1 cells. . . . . . . . . . . . . . . 170

5.10 IL-3 but not TSLP caused mast cells to develop in vitro. . . . . . . . . 172

5.11 IL-3-/- mice were protected from allergic diarrhoea. . . . . . . . . . . . 174

5.12 IL-3-/- mice were susceptible to i.v.-induced systemic anaphylaxis. . . . 175

5.13 The $i F A B P p$-IL-9 transgene enhances basal jejunal mast cell activity in IL-3-deficient conditions. . . . . . . . . . . . . . . . . 177

5.14 IL-3 is required for diarrhoea development in mice with high basal mast cell activity. . . . . . . . . . . . . . . . . . . . . 179

5.15 Lack of a single IL-3 allele causes a partial defect in allergen-induced intestinal mastocytosis. . . . . . . . . . . . . . . . . . . . . . . 181

5.16 Systemic anaphylaxis and fur matting in IL-3 sufficient and deficient mice. . . . . . . . . . . . . . . . . . . . 183

Examining milk allergenicity in Th2 immune response models 195

6.1 Dose-dependent Th2 response to cow $\beta$-lactoglobulin. . . . . . . . . . . 201

6.2 Similar Th2 response to cow and goat $\beta$-lactoglobulin. . . . . . . . . . 202

6.3 Comparison of cow and goat casein concentrations by BCA and nanodrop. 203

6.4 Similar Th2 response to cow and goat $\alpha$ s1-casein. . . . . . . . . . . . . 204

6.5 Th2 response to goat whole casein is stronger than to goat $\alpha$ s1-casein. . 205

6.6 Th2 responses to cow and goat whole caseins are similar. . . . . . . . . 206

6.7 Neither skim nor whole goat milk prevents Th2 responses. . . . . . . . 208

6.8 Goat $\beta$-lactoglobulin can induce intestinal Th2 responses. . . . . . . . . . 212

6.9 mMCP-1 levels are elevated in orally-challenged sensitized mice. . . . . 213

6.10 Cross-reactivity observed between cow and goat $\beta$-lactoglobulin but not other allergens in $i F A B P p$-IL-9 mice. . . . . . . . . . . . . . . . . . 214

\section{General Discussion $\quad 221$}

7.1 Processes leading to intestinal allergy. . . . . . . . . . . . . . . . 222

$\begin{array}{ll}\text { Appendices } & \mathbf{2 7 8}\end{array}$

8.1 References for figure 1.1. . . . . . . . . . . . . . . . . 278

8.2 Genotype testing of IL-3 and IL-9. . . . . . . . . . . . . . . . . 279

8.3 GFP and hCD2 medFI are lower in KN2/G4 cells than G4/+, KN2/+, G4/G4 and KN2/KN2 cells. . . . . . . . . . . . . . . . . . . 284

8.4 in vivo GK1.5 treatment ablates CD4 but not CD8+ T cell populations. 285 
8.5 Cytokine production by OVA or PBS-stimulated splenocytes from IL-3 sufficient and deficient mice. . . . . . . . . . . . . . . . . 286

8.6 Cytokine production by OVA or PBS-stimulated mesenteric lymph node cells from $\mathrm{Tg}+/+$, $\mathrm{Tg}-/-$ and $\mathrm{nTg}-/$ - mice. . . . . . . . . . . . 287

8.7 Mastocytosis in caprine BLG sensitized mice. . . . . . . . . . . . . . 288

8.8 Liquid stool at 60 mintes post-challenge three in intestinally atopic mice fed different allergens. . . . . . . . . . . . . . . . . . . . . . . 289 


\section{Abbreviations}

Standard International Unit abbreviations were used throughout this thesis.

$\begin{array}{ll}+/+ & \text { Wild-type mouse } \\ +/- & \text { IL-4 heterozygous mouse } \\ -/- & \text { IL-4-deficient mouse } \\ 4 \text { get } & \text { IL-4-GFP enhanced transcript } \\ 5 \mathrm{HT} & \text { 5-hydroxytryptamine; serotonin } \\ 7 \text {-AAD } & \text { 7-amino actinomycin D } \\ \text { AEC } & \text { 3-amino-9-ethylcarbazole } \\ \text { APC } & \text { Antigen presenting cell } \\ \text { ANOVA } & \text { Analysis of variance } \\ \text { anti-IFN } \gamma & \text { antibody against IFN } \gamma \\ \text { anti-IgD } & \text { antibody against murine IgD } \\ \text { anti-IL-4 } & \text { antibody against IL-4 } \\ \text { BAL } & \text { bronchoalveolar lavage } \\ \text { bBLG } & \text { cow } \beta \text {-lactoglobulin } \\ \text { BCA } & \text { Bicinchoninic acid } \\ \text { BCF-1 } & \text { IL-4 }\end{array}$

$\mathrm{BRU}$

Biomedical Research Unit of the Malaghan Institute of Medical Research

BSA Bovine serum albumin

BLG $\quad \beta$-lactoglobulin

CAE Chloroacetate esterase

cBLG (caprine) goat $\beta$-lactoglobulin

CD cluster of differentiation

CD40L CD154

cIMDM base culture medium

DAPI 4,6-diaminidino-2-phenylindole

DELTA BLAST Domain enhanced lookup time accelerated Basic local alignment search tool 


\begin{tabular}{|c|c|}
\hline EDTA & Ethylenediaminetetraacetic acid \\
\hline ELISA & Enzyme-linked Immunosorbant assay \\
\hline Flow buffer & $2 \%$ FCS, $0.01 \%$ Sodium azide in PBS \\
\hline FCS & Foetal Calf serum \\
\hline FPLC & Fast (low) pressure liquid chromatography \\
\hline FTY720 & Sphingosine-1-phosphate Receptor inhibitor \\
\hline G4 & $\begin{array}{l}\text { IL- } 4 \text { reporter allele in which GFP is produced under the control of } \\
\text { the IL- } 4 \text { promoter }\end{array}$ \\
\hline $\mathrm{G} 4 /+$ & G4 heterozygous mouse with one wild-type IL-4 allele \\
\hline $\mathrm{G} 4 / \mathrm{G} 4$ & IL-4-deficient dual G4 reporter mouse \\
\hline GFP & enhanced green fluorescent protein \\
\hline $\begin{array}{l}\text { haplo- } \\
\text { insufficiency }\end{array}$ & $\begin{array}{l}\text { Occurs when a genetic deficiency in one allele prevents development } \\
\text { of the normal wild-type phenotype }\end{array}$ \\
\hline hCD2 & $\begin{array}{l}\text { inactive human CD2 used to identify IL- } 4 \text {-producing cells in the } \\
\text { KN2 reporter }\end{array}$ \\
\hline HDM & Dermatophagoides pteronyssinus crushed house dust mite extract \\
\hline HPLC & High pressure liquid chromatography \\
\hline HS & hypersensitivity site \\
\hline ICOS & Inducible T cell co-stimulator; CD278 \\
\hline i.d. & intradermal \\
\hline i.g. & intragastric \\
\hline i.v. & intravenous \\
\hline$i$ FABP $p$-IL-9 & $\begin{array}{l}\text { IL-9 transgene inserted under the control of the Rat fatty acid bind- } \\
\text { ing protein }\end{array}$ \\
\hline $\mathrm{IFN} \gamma$ & Interferon- $\gamma$ \\
\hline $\operatorname{Ig}$ & Immunoglobulin \\
\hline Igs & polyclonal Immunoglobulins \\
\hline IL & Interleukin \\
\hline IMDM & Iscove's modified Dulbecco's medium \\
\hline IRES & Internal Ribosomal entry site \\
\hline i.p. & intraperitoneal \\
\hline $\mathrm{KLH}$ & Keyhole Limpet Hemocyanin \\
\hline KN2 & IL-4 gene knock-in human CD2 \\
\hline
\end{tabular}




\begin{tabular}{|c|c|}
\hline $\mathrm{KN} 2 /+$ & KN2 reporter mouce with one wild-type IL-4 allele \\
\hline $\mathrm{KN} 2 / \mathrm{KN} 2$ & IL-4-deficient dual KN2 reporter mouse \\
\hline $\mathrm{KN} 2 / \mathrm{G} 4$ & $\begin{array}{l}\text { IL-4-deficient reporter mouse carrying two separate IL-4 reporter } \\
\text { alleles }\end{array}$ \\
\hline $\mathrm{L}$ & Ligand \\
\hline LPS & Lippopolysaccharide \\
\hline major allergen & $\begin{array}{l}\text { protein against which more than } 50 \% \text { of food allergic individuals } \\
\text { demonstrate IgE-binding }\end{array}$ \\
\hline mMCP-1 & murine mast cell protease- 1 \\
\hline MWCO & molecular weight cut-off \\
\hline $\mathrm{Nb}$ & dead infective-phase $i$ L3 Nippostrongylus brasiliensis larvae \\
\hline $\mathrm{nTg}-/-$ & IL-3-deficient mouse sib-matched to $\mathrm{Tg}$-/- mice \\
\hline $\begin{array}{l}\text { Oral potentia- } \\
\text { tion }\end{array}$ & $\begin{array}{l}\text { post-sensitization phase of experimental allergy that precedes diar- } \\
\text { rhoea. Characterized by elevated Th2 profile, intestinal mast cell } \\
\text { infiltration and increased IgE levels. }\end{array}$ \\
\hline OVA & Ovalbumin \\
\hline PAF & Platelet activating factor \\
\hline PBS & Dulbecco's phosphate buffered saline \\
\hline PCR & polymerase chain reaction \\
\hline $\mathrm{PCV}$ & Packed cell volume; haematocrit \\
\hline PD-1 & Programmed cell death-1; CD279 \\
\hline pTh2 cell & $\begin{array}{l}\text { Th2 cell that develops in response to primary adjuvant free immu- } \\
\text { nisation }\end{array}$ \\
\hline $\mathrm{R}$ & receptor \\
\hline $\mathrm{r}$ & recombinant \\
\hline SEM & standard error of the mean \\
\hline $\mathrm{SCF}$ & Stem cell factor (cKit ligand) \\
\hline TCR & $\mathrm{T}$ cell receptor \\
\hline TFH cell & follicular T helper cell \\
\hline TGF- $\beta$ & Transforming growth factor- $\beta$ \\
\hline $\mathrm{Tg}+/+$ & $\begin{array}{l}\text { Mouse carrying the } i \text { FABP } p \text {-IL- } 9 \text { transgene and two wild-type IL-3 } \\
\text { allele }\end{array}$ \\
\hline
\end{tabular}


$\mathrm{Tg}+/-$

Tg-/-

Th cell

Th0 cell

Th1 cell

Th2 cell

Th2m cell

Th9 cell

Th17 cell

TCA

TMB substrate

Treg cell

TSLP

$\mathrm{U}$

WT
Mouse carrying the $i$ FABP $p$-IL-9 transgene and a single wild-type IL-3 allele

IL-3-deficient mouse carrying the $i$ FABP $p$-IL-9 transgene

$\mathrm{T}$ helper cell

Unpolarised activated Th cell

IFN $\gamma$ producing Th cell

IL-4, -5, or -13 producing Th cell. In this thesis, in vivo Th2 cells are considered reporter-expressing cells. in vitro Th2 cells are $\mathrm{CD} 4+\mathrm{T}$ cells that have been cultured for five or more days in the presence of IL-4.

Mast cell-inducer Th2 cell

IL-9-producing Th cell

IL-17-producing Th cell

Trichloroacetic acid

3,3',5,5'-Tetramethybenzidine substrate

Regulatory Th cell

Thymic Stromal Lymphopoeitin

Units

Wild-type 



\section{Chapter 1}

\section{General Introduction}

\subsection{Thesis overview}

Food allergy remains a life-threatening problem in society today. One in twenty children will have an allergic episode before they turn three, and the majority of these will be invoked by a common dietary protein. Over $90 \%$ of these will be induced by one of six foods: cows milk, egg, peanut, tree nuts, wheat or soy. It is still unclear why these six foods are common allergens, and further more, why only some people will develop an allergy at all. This project aimed to clarify the basic immune processes that result in allergic disease.

Research over the past hundred years has made great advances in the field of allergic disease research. The key mediators of the allergic response have been elucidated; the cell types involved in the response are known; and a number of risk factors have been identified. However, our current understanding of the allergic process is inadequate. We don't know the first steps that lead to allergic sensitization; we are not able to predict with reasonable certainty which children will become allergic; we don't know how to prevent an allergy from developing; and most critically, we don't know how to turn an established allergy off.

This thesis begins with a general introduction into the field of allergy. In this introduction, knowledge of the role of mast cells and the immunological processes that underlie allergic sensitivity are outlined. Some of the epidemiology of allergy is covered and is also used as evidence that supports the concept of intrinsic allergenicity, which is subsequently investigated for some common allergens. As interleukin(IL)-4 producing, CD4 + T helper type 2 (Th2) cells are heavily implicated in the allergic process and are a major focus of this thesis, their development and contribution to the allergic process are discussed in depth. Chapter two provides details of the meth- 
ods that were used during this project. Chapters 3-6 present the project findings regarding Th2 cell development and function in allergic immunity.

The focus on the Th2 cell arose from previous studies in our lab that broke the historically accepted view that IL-4 is required to initiate Th2 cell development[1]. From these earlier studies, questions pertaining to the true contribution of IL- 4 to Th2 cell development naturally evolved and were accordingly investigated. These studies, in turn, gave rise to the question of how genetic defects in IL-4 production affect not only Th2 cell development, but also their function. These concepts are the focus of Chapter four. Chapter five aims to clarify the role of CD4+ T cells in the post-sensitisation phase of food allergy, and in the final results chapter, a series of tests are performed that aim to clarify why goats milk is a less allergenic food than cows milk. Future directions and potential implications of our findings are covered at the end of each chapter, and a general overview of the concepts contained within are discussed in Chapter seven.

My dissertation covers the reasons that I think allergies are still problematic. It outlines our attempts to clarify unknown facets of Th2 cell development and function, and in this, specifically addresses some fundamental aspects of the allergic process. Understanding the immunological processes that lead to the physiological allergic response has great potential to reduce the burden of allergic disease in society today. 


\subsection{Allergy and food induced allergic reactions}

\subsubsection{What is allergy?}

Allergy, or type I hypersensitivity, is an acute adverse immune response to a generally innocuous protein or substance. The current paradigm of allergy links back to the $1800 \mathrm{~s}[2]$, with the first direct tests carried out 100 years ago in dogs[3]. Portier and Richet[3] determined that when dogs are immunised with two additively non-lethal doses of poisonous Physalia man o' war tentacle extract, if the second injection is given two weeks after the first, the dogs will collapse, develop cyanosis and in many cases, die. These studies gave rise to the concept that underpins allergy as it is understood today. More general allergic symptoms include vomiting, diarrhoea, wheal and flare, urticaria, wheezing and bronchospasm and some or all of these symptoms can be triggered simultaneously during an allergic response[4-6]. Fatal allergic anaphylaxis is usually attributed to complications involving the respiratory and circulatory system, caused by either hypovolaemic systemic shock and inadequate organ perfusion or difficulties with breathing[5-8]. Food allergy is defined as an adverse immune response to food $[9,10]$. To manage an allergy, people are advised to practise allergen avoidance[10, 11]. However, the rapid onset and potential severity of an allergic episode make it necessary to develop new strategies to deal with allergies. This is particularly important because one in four cases of fatal anaphylaxis is caused by unintended allergen ingestion, and immediate adrenalin administration, which is the best current intervention, does not prevent death in every case[7, 8]. There is currently no treatment available that is entirely sufficient for the prevention of anaphylactic death $[5,7,8]$. Food allergy is thought to be increasing in prevalence[9, 10, 12] and understanding ways to subvert the immune processes that precede allergic responses are key directions of current medical research.

\subsubsection{IgE is at the centre of allergic disease}

Food allergies are distinguished from food intolerances because an allergy is caused by an active immune response against a specific food[10]. Food intolerances can be 
caused by specific food components, but are not immune mediated. For example, an inability to absorb and breakdown fructose or lactose is associated with gastrointestinal discomfort and diarrhoea that disappears with elimination of the sugars from the diet[13-15]. Toxins in foods and pharmocologically active molecules like caffeine can also cause food allergy-like symptoms[5]. The food allergy response is also acute, which helps distinguish it from other chronic forms of gastrointestinal disorder, like inflammatory bowel disease or functional diarrhoea[16-18]. The current view of allergy is that on first allergen exposure, either in utero or soon after birth[19, 20], sensitization occurs such that subsequent exposures can trigger an allergic response. The culmination of the sensitization phase is the generation of allergen-specific Immunoglobulin E ( $\operatorname{Ig} \mathrm{E})$ antibody. Fifty-sixty percent of food allergies can be attributed to IgE dependent processes[11, 21, 22].

$\mathrm{IgE}$ was first discovered and designated as the reaginic antibody in a series of studies by Ishizaka and Ishizaka[23-25]. In their studies, serum from people with ragweed pollen allergy was depleted of the major known antibody isotypes (IgM, IgD, IgG and $\operatorname{IgA}$ ) and shown to retain its ability to passively sensitize skin[23, 25]. These investigations further determined that it was possible to remove the reaginic activity from $10 / 10$ studied sera by depleting a component that specifically recognised the ragweed allergen[25]. This component had detectable antibody light chains but none of the $\operatorname{IgM}, \operatorname{Ig} \mathrm{D}, \operatorname{IgG}$ or IgA isotypic regions, and had distinct electrophoretic properties to the other antibody isotypes[23, 24, 24]. The isotype class was proposed and accepted as $\gamma \mathrm{E}$ antibody, or $\operatorname{IgE}$.

Although the specificity of the allergic response is provided by $\operatorname{IgE}$, the allergic response is caused by vasoactive mediators released by IgE-bound mast cells and basophils. IgE is bound to the surface of mast cells and basophils by direct interactions with the alpha subunit of the high-affinity IgE receptor, FceRI[26-29]. When cell-bound IgE is ligated by allergen, the cells release granules containing vasoactive mediators that cause the allergic symptoms [5, 30]. Clinical allergic symptoms are generally more severe, and allergies are far less likely to resolve in individuals with high titre $\operatorname{IgE}[31-35]$. In experimental systems, the severity of the allergic response correlates with the amount of IgE and level of mastocytosis[36-39]. In some murine experimental systems, alternate cell types and an alternate antibody isotype, IgG1, 
are now known to play important roles in antigen-specific anaphylactic and allergic responses[40-42]. However, IgE-mediated experimental anaphylaxis initiated by the intravenous or the oral route is attributed to mast cell activity[37, 43, 44]. Therefore, $\mathrm{IgE}$ and mast cells are central to the allergic response.

\subsubsection{Physiological changes that occur during systemic ana- phylaxis}

The vasoactive mediators released by mast cells are diverse. These include prostaglandins, leukotrienes, mast cell proteases, histamine, serotonin and platelet-activating factor (PAF) $[5,30,37,45]$. Histamine and proteases are preformed mediators, whereas PAF, leukotrienes and prostaglandins are synthesised after activation[30]. Appropriately activated mast cells are also reported to produce a number of cytokines, including Interleukin (IL)-4 and IL-13[30]. The mediators generally act in a co-ordinated fashion, but some manifestations of allergy can be prevented by treatment with specific mediator antagonists and this has been examined in terms of the inhibition of the physiological allergic response.

Experimental systemic anaphylaxis induces measurable physiological changes mediated predominantly by histamine and PAF. IgE-mediated mast cell-dependent[43] systemic anaphylaxis causes smooth muscle contraction and vascular leak[46, 47], resulting in loss of plasma volume, loss of blood pressure and a drop in core body temperature[43, 48]. Isotonic fluid perfusion of anaphylactic mice is sufficient to prevent haemaconcentration, ameliorate temperature loss and prevent anaphylactic death[48], indicating that hypovolaemia is a major cause of anaphylactic death in mice. The systemic drop in temperature in mice can be prevented by administration of anti-histamines or combined histamine and PAF antagonism, as the temperature nadir is reduced and vascular leak is reduced when these pathways are inhibited[37, 48, 49]. Conversely, serotonin inhibitors, and 5-lipoxygenase inhibitors that prevent prostaglandin synthesis are unable to prevent allergen-specific systemic anaphylaxis in mice[37, 49]. This indicates that histamine and PAF mediate the systemic allergic reaction. Histamine- and PAF-induced anaphylaxis are also exacerbated by IL-4 and IL-13[48, 50]. Additionally, PAF-induced anaphylaxis causes more 
haemaconcentration when the amount of mediator introduced induces equivalent hypothermia, suggesting that distinct mechanisms may be involved in each part of the anaphylactic response[48]. However, both PAF and histamine can cause anaphylactic death and are important for mast cell- and IgE-mediated experimental systemic anaphylactic responses.

Currently, there is no universal clinical definition of anaphylaxis[4, 6]. In humans, anaphylaxis is generally considered severe when cyanosis is observed, when the individual collapses, or when soluble oxygen levels in the blood fall below 92 percent saturation[6]. More general changes include a drop in blood pressure, and a range of physical symptoms indicating respiratory or gastrointestinal involvement [4, 6]. Intramuscular adrenalin administration is the best recommended treatment for anaphylaxis[6], and its delayed or improper administration is a common cause of anaphylactic death $[7,8,10,11]$. One of the most frequent presentations of food allergy, and a secondary diagnostic feature of anaphylaxis, is diarrhoea[4] and some of the physiological mechanisms of allergic diarrhoea induced by intestinal allergen exposure have been investigated.

\subsubsection{Physiological processes during fasting and allergic in- testinal responses}

The normal physiological motility of the intestine is disrupted during an intestinal allergic response. In the fasted state, the major contractions of the intestine are periodic coordinated phasic contractions that begin in the stomach and pass along the length of the small intestine to maintain uni-directional gastric transit[51]. This process, called peristalsis, occurs in most mammals[51]. Introduction of allergen to the small intestine of sensitized rats disrupts fasting peristaltic movements and induces a rapid increase in phasic contractile frequency of both smooth muscle layers along the entire length of the small intestine, causing increased allergen transit through the small intestine $[52,53]$. Similarly, when specific allergen is deposited onto colonic sections from sensitized rats, a tonic contraction of both smooth muscle layers is induced and the colonic contents are transported more rapidly in vivo[44, 54]. Not only is motility increased, but net sodium, chloride and water absorption are impaired and 
concomitant active luminal secretion of water and ions increased following allergen exposure[55, 56]. Oral allergen exposure in sensitized mice also causes acute loss of blood plasma volume analogous to that occuring during systemic anaphylaxis in sensitized intravenously-challenged mice[48, 57], suggesting that the net change in intestinal fluid secretion can affect homeostatic blood volume as well. Collectively, these processes expedite allergen transit and ultimately result in profuse watery diarrhoea soon after intestinal allergen exposure[37, 44, 53, 58, 59].

The processes occurring in allergic humans are similar to those in experimental systems and have been concisely summarised by Hugh Sampson[60]. In the stomach of allergic individuals, allergen transit is slowed, but once in the small intestine, intestinal motility is enhanced, peristalsis is interrupted, intestinal vasodilatation and mucus hypersecretion occur[60]. Diarrhoea and sometimes vomiting follow[5, 60]. Mice and rats are non-emetic species[61] so diarrhoea is the major presentation of intestinal allergic responses in rats and mice. Much of the physiology of the intestinal allergic response is known.

\subsubsection{IgE and mast cells mediate intestinal allergen-induced allergic responses}

Investigations have demonstrated that intestinal allergic responses are IgE- and mast cell-dependent. Mast cell degranulation is observed upon intestinal allergen exposure[37, 54]. The response is mediated by IgE, as demonstrated by colonic contractile responses being specific for the sensitising antigen and passively transferrable to naive intestinal sections via immune sera, but like passive skin immunisations[62], this activity is lost if sera is first heat-inactivated[44, 54]. Similarly, treating mice with an antiIgE antibody, ablating mast cell (and the intestinal pace-maker interstitial cells of Cajal) populations with anti-cKit antibodies, cKit-inhibiting drugs[63] or monitoring allergic disease development in FceRI-deficient mice, all result in reduced diarrhoea incidence[37]. In humans, positive oral food challenges also cause intestinal mast cell degranulation and in greater than 95 percent of cases this correlates with antigenspecific skin prick sensitization[60], indicative of antigen-specific IgE sensitization. Collectively, the data provide strong evidence that oral allergen-induced diarrhoea is 
mast cell- and IgE-mediated.

The mast cell products that cause diarrhoea differ from those that cause systemic anaphylaxis. Colonic circular muscle contraction can be induced by exogenous administration of a range of mast cell vasoactive mediators: 5-hydroxytryptamine (5-HT; i.e., serotonin) and leukotriene D4 are particularly potent, and contractility can be partially induced by histamine, PAF or prostaglandin D2[44]. However, the antigenspecific response of both smooth muscle layers along the entire length of the intestine is prevented by mast cell stabilization with doxantrazole, and some responses with disodium cromoglycate, but histamine antagonists have no effect[44, 54, 64, 65]. Leukotriene D4 antagonists and PAF blockade prevent contraction of both layers of the colon in the rat $[44,54]$, whereas, 5-HT1 and 5-HT2 receptor blockade and indomethacin, which inhibits the cyclooxygenase pathway and inhibits prostaglandin synthesis, prevent allergen-induced jejunal smooth muscle contractility[64, 65]. Additionally, in live animals, prostaglandins and serotonin have been implicated in altering jejunal contractility by modifying neuronal innervation[52]. Concomitant blockade of multiple mediators is the best means to inhibit antigen-induced colonic contraction and diarrhoea[37, 54]. Specifically, concomitant blockade of PAF and serotonin can inhibit allergic diarrhoea in mice[37]. In addition to these vasoactive mediators, cytokines can also influence the intestinal allergic response: IL-4 and IL-13 directly enhance intestinal smooth muscle contractility[50, 66]. IL-4 can also affect intestinal ion flux and tissue responsiveness to mast cell vasoactive mediators[50]. Thus, while histamine is a key trigger for systemic anaphylaxis, histamine- and histamine receptor-antagonists do not prevent intestinal anaphylaxis, whereas, PAF, serotonin, leukotrienes and prostaglandins are implicated in the intestinal response[37, 47, 54].

In summary, while both intestinal and systemic allergic responses are mediated by mast cells, and systemic and intestinal anaphylactic responses are exacerbated by IL-4 and IL-13[48, 50, 66] when antigen is deposited in the intestine, distinct mediators are implicated in the response. The similarities and differences that are attributed to systemic and intestinal anaphylactic responses are summarised in figure 1.1. Histamine and PAF are key to the systemic anaphylactic response; PAF, serotonin, and potentially prostaglandins and leukotrienes are involved in intestinal responses[37, 52, 64]. 
There is also evidence that systemic and intestinal manifestations of disease can exist independently of each other, even when allergen is administered by the oral route $[37,67,68]$. Therefore, it is possible that the immunological mechanisms that permit allergic responses in the intestine are distinct from those that drive systemic anaphylaxis. 


\begin{tabular}{|c|c|c|}
\hline Anaphylaxis & Response: & Major Mediators \\
\hline Systemic & $\begin{array}{l}\text { Vascular smooth muscle contraction }{ }^{1} \\
\text { Vascular leak } \\
\text { Plasma volume } \downarrow^{3,4} \\
\text { Blood pressure } \downarrow^{4} \\
\text { hypoxia }^{5}\end{array}$ & Histamine + PAF \\
\hline Gastrointestinal & 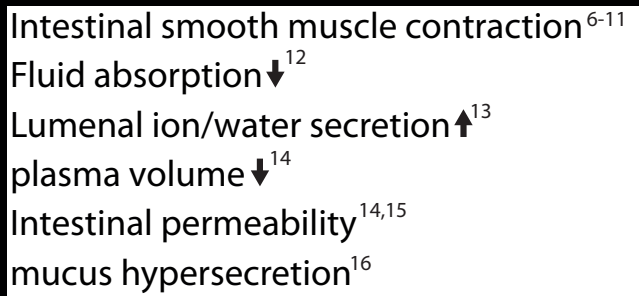 & $\begin{array}{c}\text { PAF + Serotonin } \\
\text { prostaglandins \& leukotrienes }\end{array}$ \\
\hline
\end{tabular}

Figure 1.1: Symptoms and mediators of systemic and intestinal anaphylaxis. Mast cell-dependent responses that result in systemic or intestinal (not including the stomach) allergic responses in rodents and/or humans. Superscript numbers denote references, which are listed in the table-specific bibliography in the appendices. 


\subsubsection{Management of food allergy}

The current advice given for the prevention of allergic responses in sensitized individuals is allergen avoidance[10,11], however, a number of alternative strategies are also used. Therapies can target end-stage effects, for example, interrupting the signaling of the products of mast cells and basophils such as the cysteinyl leukotriene and histamine antagonists monteleukast and loratidine[69] or aim to inhibit mast cell degranulation using local administration of disodium cromoglycate[70, 71]. Clinicians have also explored the use of allergen desensitization by giving escalating doses of allergen sublingually, orally or subcutaneously[72-74]. Desensitizing immunotherapy can take months to years to be effective and is generally incomplete, however, treatment usually increases the tolerated allergen dose and reduces the severity of the allergic symptoms evoked by chance or experimental allergen exposure[74, 75]. During the desensitization process, patients can experience mild allergic symptoms[75] and a rare but major risk of desensitization therapy is allergic anaphylaxis[76]. To limit the risk of anaphylaxis during immunotherapy, desensitization strategies have explored, with some success, the use of peptide fragments of allergens, rather than whole allergens for therapy[77]. One of the most promising therapies to date uses allergen non-specific anti-IgE antibodies. This approach has reduced the severity and incidence of allergic responses and allergic asthma in the clinic setting[78-81] and is also shedding light on the processes that permit and perpetuate allergic responses once established[82-85]. While allergic responses in the lung are not a focus of this thesis, allergic asthma also affects a number of New Zealanders, and thus its management deserves a brief mention. To manage allergic asthma, inhaled glucocorticosteroids and when necessary, leukotriene modifiers and $\beta 2$ adrenergic agonists are the most commonly prescribed preventative therapies, and $\beta 2$ adrenergic agonists are also used to reverse acute asthma attacks[81]. These, however, do not reverse sensitisation, and aim to limit the severity and frequency of allergic asthma. The major current therapies, therefore, try to reverse sensitization or diminish the effects of the mast cell products. To date no single treatment has had complete success and each has associated maladies and contraindications. Understanding the steps that lead to sensitization and perpetuate allergies is key to reducing the incidence of allergic disease. 


\subsubsection{Identification of food allergy}

Not only are food allergies difficult to manage, they are difficult to distinguish from other gastrointestinal disorders. Some diseases exist that present with similar symptoms to food allergies[86-89] and cases have been identified in which the response is likely to be IgE-mediated but systemic IgE and peripheral disease manifestations are not detected[90]. The identification of a specific causative food allergen is made even more difficult as the majority of allergic individuals are sensitized to multiple foods and often to multiple proteins contained in a single food[32, 33, 35, 91, 92]. This can make it difficult to appropriately exclude the allergen without unneccessarily restricting the diet, which is especially important for infants where dietary exclusion could significantly impact on normal development and growth[86, 93]. Thus, identifying the presence of a food allergy, particularly in the paediatric population, requires careful evaluation.

Standardised processes for food allergy determination are improving disease identification. IgE assays with high positive predictive values have now been established for some major allergens in the US, including milk and egg[31]. However, allergies to other foods still benefit from confirmation by the double-blind placebo controlled oral food challenge test. As self/parentally-reported food allergy incidence is inflated by several-fold[11], it is now advised that before allergen elimination is trialed, food allergies are diagnosed based on a combination of medical history, skin-prick testing, serum antigen-specific IgE titres and when it is not ill-advised, a double-blind placebo controlled oral food challenge[11, 94]. Thus, simple quantitative disease readouts enable rapid identification of potential allergens and support the qualitative observations used to identify a specific food allergy.

\subsubsection{Hereditary risk factors for food allergy}

Numerous risk factors for the development of allergic disease have been identified[19, 95]. Current reviews of risk factors[19, 95] and genes associated with allergy and asthma[96] are available.

First, there are hereditary and genetic predispositions. Allergies, asthma and eczema 
encompass the atopic disorders[97], and are associated with polymorphisms in some genes. Generally, the interaction of a number of genes with low penetrance are thought to compound to increase disease risk, and these are revealed in carefully controlled, genome-wide association studies[96]. For example, high frequencies of single nucleotide polymorphisms in the $I l 4$ or IL-4R $\alpha$ genes are associated with increased risk or severity of asthma and allergic disease[98-100]. There are also specific mutations that can be associated with disease phenotypes. For example, loss-of-function mutations in the filaggrin gene, which is associated with epidermal barrier defects, predisposes infants to eczema, and increases risk of asthma and allergic rhinitis[19, 101, 102]. People of certain ethnicities, like african americans, are more likely to develop allergies than caucasian americans[19, 103]. Collectively, allergies are considered hereditable[104], but there is significant gene-environment interaction that makes it difficult to predict disease development and the specific type of atopic disease that will present[96].

\subsubsection{Diet and the intestinal microbiota influence disease risk}

A risk factor that is not limited to the allergic disease setting, relates to the intestinal microbiota. A balanced and diverse intestinal microbial community protects from numerous diseases. Reduced intestinal microbial diversity in infants is associated with increased risk of allergic disease[105], and diet supplementation with selected probiotics has been associated with reduced incidence of allergies[106, 107]; similarly, drinking unpasteurized milk is associated with reduced skin prick test positivity in farm children[108] and this may relate to the effect of raw milk on intestinal microbiota composition. In terms of early-life exposure, birth by casearian section has, in some cases, been shown to be a risk factor for allergic sensitization[109, 110]. It has been suggested that this may be influenced by the type of microbes to which the infant would be exposed through the birth canal (i.e., vaginal and intestinal commensals), or without it[109-111]. Treating mice with antibiotics has been shown to alter the diversity and composition of the resident intestinal microbiota for long periods[112], and also to promote IgE production, basophil hyper-responsiveness and to increase allergic airways inflammation in mice[113]. The resident microbiota is now considered to significantly influence the intestinal immune system, and examining the changes in 
population dynamics that are observed, and potentially influence human health and disease will continue to be an important focus for balanced human health.

Nutrition is both affected by, and thought to affect allergic disease progression. Aside from the obvious, that exclusion of allergens from the diet improves infant growth[114], levels of essential dietary metabolites, like vitamin A and D are associated with allergic disease risk[115-118]. Food allergies are associated with numerous subsequent developmental problems, including rickets, failure-to-thrive and some psychosocial maladies[114, 119-121]. More recently, the links between diet, microbiota and allergictype immunity has been linked: Maslowski et al.[122] demonstrated that the shortchain fatty acids released by the break-down of dietary fibre by the intestinal microbiota limit allergic and enteric disease severity[122]. Thus, nutrition and commensal microbiota are inter-related disease risk factors.

\subsubsection{Environmental exposure as a risk factor for allergy}

There are also environmental risk factors involved in the development of food allergies. In a seminal study David Strachan showed that family position influenced the risk of developing an allergic disease and argued that this might relate to a 'dirty' environment[123]. Sensitization to environmental allergens has been associated with the levels of allergens found in the home[124]. Similarly, environmental sensitization has been proposed as a potential mechanism for sensitization to stable food allergens. This follows evidence that demonstrates an association between eczema, asthma, and allergic disease. Infants who develop atopic dermatitis at a young age are at increased risk of developing asthma and some forms of allergy in later life[97]. In a series of studies, Lack et al. examined the incidence of peanut allergies in infants in early life. In these studies, oral ingestion of peanuts was associated with protection from disease, whereas high household consumption in infants not fed peanuts, or exposed to peanut products by skin application through moisturisers of eczema creams, were associated with increased risk of peanut sensitization[125-127]. These data suggest that environmental, rather than intestinal exposure to some stable allergens may increase the risk of food sensitization; however, these studies are not all-encompasing. Maternal exposure to house dust mite allergens during pregnancy is associated with increased 
cord blood IgE levels[128], which indicates that sensitization may occur in utero. The range of potential mechanisms of sensitization have lead to the removal of the advice that allergens should be avoided, in both the USA and the UK[19, 129].

\subsubsection{Oral tolerance}

The default response to ingested food proteins is oral tolerance. Oral tolerance is an active regulatory immune process that acts to reduce subsequent immune responses to specific antigens[130]. Feeding mice with OVA prior to sensitisation, either by gavage or indirectly through the breastmilk prevents sensitization and the subsequent generation of allergic responses in the intestine or lung of mice[130-136]. In humans, similar observations have been made. In an oral feeding study, Husby et al.[137] immunised individuals subcutaneously with keyhole limpet hemocyanin (KLH), half of whom had previously been fed numerous low doses of KLH. When individuals were subsequently challenged with KLH, IgM, IgG and IgA antibodies and antibody-secreting cells were detected systemically, but individuals that had ingested KLH eventually demonstrated diminished $\mathrm{T}$ cell proliferative responses and reduced frequencies of delayed-type (type IV) hypersensitivity reactions[137]. KLH can also induce IgE-mediated responses in humans, because skin wheal-and-flare responses can be induced by intradermal KLH injection in healthy and atopic individuals, if they are first immunised with KLH and alum[138]. However, oral exposure in some circumstances can result in sensitization. If mice are fed with protein in the presence of cholera toxin, this will result in IgE generation, intestinal mast cell infiltration and allergic sensitization permissive of allergic responses[139, 140]; guinea pigs can be sensitized through oral exposure in the absence of adjuvant[141]. In mice and humans, ingestion of proteins usually induces active immune processes that suppress subsequent immune responses[130, 142]. Therefore, the development of an allergy involves not only sensitisation, but a corresponding break in oral tolerance.

Work has investigated the processes involved in oral tolerance. Treg cells (that suppress other $\mathrm{T}$ cell responses) are involved in oral tolerance in rodents[131, 134, 143]. Similarly, Grindebacke et al.[144] recently demonstrated that for the first 18 months of life, Treg cells express the intestinal homing molecule $\alpha 4 \beta 7[142]$ at in- 
creased frequencies[144], indicating that the infant immune system may be preconditioned to support oral tolerance. The uptake and processing of the food proteins is also thought to be important. Particulate antigens, like bacteria and caseins are taken up by specialised M (microfold) cells in the follicle-associated epithelium overlying Peyer's patches[130, 139, 142]. These cells take up antigen and deposit the particulate matter, undigested, in an invagination below the $\mathrm{M}$ cell for uptake and presentation by other antigen presenting cells. This processing is thought to contribute to the tolerance process to intestinal bacteria[130]. Dendritic cells in, and directly beneath, the intestinal epithelial layer can extend dendrites into the intestinal lumen to sample antigens[142, 145]. Tolerance to soluble antigens involves dendritic cell antigen carriage[130], their migration to the mesenteric lymph nodes and induction of Treg cells that could subsequently migrate into the intestinal lamina propria[130, 134, 142, 143]. IL-10 producing T cells in Peyer's patches are also implicated in oral tolerance[146, 147]. There are also some other potential mechanisms of tolerance generation. Epithelial cells in orally fed naive rats have also been shown to produce antigen-loaded MHC-II 'tolerosomes' that disperse systemically and inhibit delayed-type hypersensitivity reactions[130, 142, 148]. This tolerizing capacity was lost if it was first MHC-II-depleted[148], suggesting that the tolerosomes specifically were required for tolerance development. Further, depletion of antigen by removing antigen-bound MHC-II is not a sufficient explanation for the loss of activity. While allergen is absorbed systemically following oral ingestion[149], and can induce allergic responses in passively-sensitised skin[150], the tolerizing capacity of serum does not relate to the amount of antigen contained in the transferred serum[151]. Therefore, in addition to specialised antigen presenting cells that are implicated in the generation of Treg cells, systemically dispersed tolerosomes may also contribute to the oral tolerance process.

Collectively, while much is known about the disease phenotype, the underlying physiological mechanisms that cause the allergic response, and the factors that increase disease risk are being elucidated, current treatments are inadequate. Better understanding of the allergic disease process is required to improve quality-of-life for allergy sufferers. Many allergic responses require the coordinated interaction of $\operatorname{IgE}$, allergen and mast cells, so factors that promote allergic sensitization, beginning with the role 
of the allergen itself, in the allergic process, are important. 


\subsection{Allergenicity and the epidemiology of allergic disease}

\subsubsection{Allergy and allergenicity}

Proteins that have been identified as allergens in five or more people are now classified by the World Health Organization and International Union of Immunological Societies Allergen Nomenclature Sub-committee. Allergens are named based on the source species, and the order in which they were identified[152, 153]. For example, bovine serum albumin was the sixth identified allergen sourced from the common dairy cow Bos taurus, formerly Bos domesticus, and is referred to in the allergen nomenclature as Bos d 6. All classified allergens are listed at http://www.allergen.org[152]. This and similar allergen databases are proving useful in the identification of common properties of allergens[154].

In terms of what substances evoke allergic responses, not all proteins are equal. In 2008, Radauer et al.[155] observed that when allergens, including food, environmental and industrial allergens, are classified by structure only $2 \%$ of structural families contained allergens[155]. An equivalent distribution of randomly selected proteins from the same source taxa incorporated $5 \%$ of families[155]. The findings of Radauer et al. provide evidence that some allergens share common structural features. This was further evidenced in their analyses because specific protein families were enriched for allergens. Prolamins, profilins and EF hand domain-containing proteins were the most common plant allergens; tropomyosins, EF hand domain-containing proteins, lipocalins and PR-1 proteins were the most common animal-derived allergens[155]. With similar observations made by other groups[154], the findings of Radauer et $a l .[155]$ suggest that there is some intrinsic feature that makes a protein or food more likely to be an allergen.

This intrinsic property is not simply proportional to the amount of protein to which an individual is exposed. Small contaminants in highly purified proteins can provide the major sensitizing capacity of a food protein[156]; autoadjuvant properties of allergens can contribute to their sensitizing potential[157, 158], trace and non-protein antigens can evoke allergic responses [91, 158]; and cooking and sterilization processes can alter an allergen, rendering it innocuous or more hazardous[139, 159-162]. For example, 
pasteurizing purified milk proteins increases the severity of the allergic response they induce in mice[139], but baked products containing milks are often well tolerated[162]. It is important to keep in mind that while studies like those of Radauer et al.[155] indicate some protein structures are more likely to be found in allergens, every food has the potential to be an allergen[10]. Similarly, it is thought that certain protein folds or structures are more likely, rather than are the only folds that are immunogenic and/or allergenic[163]. The term 'allergenicity' was coined to describe the intrinsic capacity of a substance to sensitise and trigger an allergic response. Allergenicity conveys a sense of the 'relative allergic risk' of a given protein or food, indicating its likelihood of being problematic in allergy-susceptible individuals[164]. Unravelling what gives an allergen allergenic properties has been a key question for a number of years[104], and is likely to continue as an important question for a number of years to come.

\subsubsection{Molecular properties of allergens, with specific refer- ence to food allergens}

Numerous excellent reviews regarding the common features of allergens are available, and these identify some general properties common to food allergens (e.g., [153, 154, 164]). First, food allergens are usually small proteins, ranging in molecular weight from $10-70 \mathrm{kDa}[153,164,165]$. Bovine beta-lactoglobulin is an $18 \mathrm{kDa}$ protein[56]; the egg allergens OVA and ovomucoid are 45 and $28 \mathrm{kDa}$, respectively[156]. However, the almond storage protein amandin (Pru du 6), is a hexameric protein with molecular weight estimates of 200, 360 and $427 \mathrm{kDa}[166-168]$, and bovine immunoglobulin (Bos d 7) when fully assembled is $160 \mathrm{kDa}$ and is ingested in milk[169]. Thus, there are examples of large food allergens.

One often overlooked feature of food proteins in general, is that proteins generally evolve with a purpose. Many allergens have been characterized as possessing a specific biological function, such as proteolytic activity[154]. Taking the most common allergen structures determined by Radauer et al.[155] as examples, plant allergens were enriched in the profilins, EF hand domain and tropomyosin families[155]. Profilins are involved in the regulation of signal transduction and actin polymerisation, and some 
of these are food allergens[154]; polcalins are two EF hand-containing calcium-binding proteins that can be aeroallergens[154, 170, 171]; Tropomyosins are common food allergens, and are muscle proteins found in invertebrates[154]. Some food allergens are energy storage proteins, like albumins[154] and the almond allergen amandin (Pru du 6) $[166,168]$. Nonspecific lipid transfer proteins cause cross-reactive fruit allergies to peach and apple[154, 172, 173] and were identified[155], and the lipocalin BLG binds a number of molecules, including fatty acids, cholesterol and vitamin D2[174-177]. Whether fatty acid transport is its natural function is not established[174, 175]. However, proteins with protease and enzymatic function, calcium, ligand- or metal-binding activity are enriched in allergens and this is also true of food allergens[154, 155].

The third property common to many food allergens is that they are often resistant to proteolytic digestion at acidic $\mathrm{pH}[153,178]$. Proteolysis-resistant proteins are typical class 1 food allergens[153], and can trigger responses in the intestine. When incubated in simulated gastric fluid at low pH (less than 3), ovalbumin (Gal d 2), beta-lactoglobulin (Bos d 5) and the apple allergen Mal d 2 all remain intact for at least sixty minutes[178-180]. It has been shown that the IgE binding capacity of BLG can be removed if it is de-stabilised and rendered susceptible to digestion[181]. The apple allergen Mal d 3 that is stable to proteolytic digestion causes cross-reactive fruit allergies[154, 172, 173]. Many response-evoking food allergens are therefore resistant to digestion.

Again, while resistance to digestion is a property of some food allergens, digestibility does not preclude a food from being an allergen. Food proteins that are digested by pepsin in less than two minutes, like caseins[178], can be major allergens in humans[91, 182, 183]. After extensive incubation of protein in digestive enzymes, antigenic epitopes can remain intact[184]. Foods can also contain both digestible and undigestible allergens[180, 185] and cross-reactive allergens from different food sources can be differentially sensitive to gastric digestion[186]. Class 2 food allergens are allergens that do not have sensitizing capacity, but are cross-reactive with aeroallergens[153]; the class 2 apple allergen Mal d 1 is readily degraded by pepsin at gastric $\mathrm{pH}[186]$, but can still trigger allergic responses in the upper gastrointestinal tract, skin and face[187]. Additionally, gastric transit time and $\mathrm{pH}$ are not always slow and low, and can also be compromised. For example, in newborns, the average gastric 
transit time is short (around 25 minutes[188]) and their gastric $\mathrm{pH}$ is generally higher than in adults (pH 5 or more[189]). Proton pump inhibitors that prevent gastric acid secretion and raise the stomach $\mathrm{pH}$ are also prescribed to some infants[114], which could limit the opportunity for gastric enzymes to cleave ingested proteins. In terms of the allergic response, these processes could result in 'digestible' allergens passing through to the small intestine intact. However, not all individuals have allergic responses; clearly, exposure to a highly allergenic, stable protein does not automatically render an individual allergic. Understanding not only the features of the allergen that permit allergies, but also elucidating why only some individuals become sensitized requires further investigation.

The many exceptions to the general features of a food protein that can make it allergenic suggest that we do not fully comprehend what contributes to allergenicity. In part, I think that under the appropriate inflammatory conditions, any protein could be rendered allergenic Adjuvants are employed with the intention of increasing the immunogenicity of antigens used to immunize and similar adjuvanticity effects could be argued to increase allergenicity. Even in these contexts, however, I would expect the longevity of the induced allergy-type immune response to differ between common and uncommon allergens I think the immune system can sometimes work out when its response is misdirected. As such, rather than discrete populations of allergens and non-allergens, food proteins are likely to comprise those that are high-risk for allergy and those that are of lower risk. As multiple factors contribute to the development and maintenance of allergic sensitization (e.g., exposure frequency, exposure mass, route of first exposure, age of first exposure, inflammatory environment surrounding first exposure, route of second exposure), examining the ability of foods to induce allergic immunity, as well as the host-derived and environmental factors that increase the likelihood of sensitization and/or an allergic response need to be explored synchronously and considered together.

\subsubsection{The epidemiology of food allergy}

The allergenicity of food proteins has largely been established from epidemiological data. Similar to the restricted set of protein families that contain most allergens, most 
food allergies can be attributed to a small number of allergens[60]. Food proteins have different likelihoods of sensitising infants, and have different resolution rates as children age $[9,33,35,190]$. During infancy, milk allergies account for half of all food allergies, occurring in $2.5 \%$ of children, while the next most frequent allergen, egg, causes a response in $1.5 \%$ of children[9]. For milk and egg allergies, 70 to $80 \%$ resolve by the age of sixteen[4,33,35]. While less frequent[9], soy allergies follow a similar prognosis, with $70 \%$ resolving by age ten[32]. In contrast, although allergies to peanuts, tree nuts, fish and shellfish are also less frequent in infants[9], they are unlikely to resolve[4]. Allergies that persist into adulthood are associated with high $\operatorname{IgE}$ levels$[32,34]$ and for milk, peanuts and eggs, allergen-specific IgE titres with greater than $95 \%$ positive predictive value for an oral response have been determined empirically in some clinics[31].

\subsubsection{Sensitization to multiple foods}

An allergy to one protein is also highly likely to co-present with allergies to other foods[191] and this is a particular problem in milk allergic infants. While most milk allergies resolve, one major complication for milk allergic infants that cannot be breastfed, is that there are few alternatives for feeding. In cow milk allergic individuals, cross-sensitization to other milks is common[169, 191]. Greater than $90 \%$ of cow milk allergic infants are cross-sensitized to goat milk[192], 40\% of milk allergics are cosensitized to soy[35], and $80 \%$ of soy allergic infants are also allergic to cow milk[32]. There are also rare cases of IgE cross-reactivity to homologous human milk proteins in cows milk-allergic infants[193-197]. Often, the best nutritional source for milk allergic infants is an extensively hydrolysed milk-based formula or a synthetic single amino-acid formula[114, 120]. Although they have poor palatability and different physico-chemical composition to normal milks[93], they are considered nutritionally complete. Further, the makeup of milks from different species is also different[169]. Food allergies, particularly milk allergies, during infancy may put infants at risk of nutritive deficiencies[121], failure to thrive[60, 93, 120] and impact the quality of life of allergic individuals and their families $[97,119]$. Elucidating the molecular changes that result in sensitization, particularly milk protein sensitization, may provide novel 
ways to interrupt the allergic process.

\subsubsection{Atypical mechanisms of sensitisation}

While epidemiological data shed some light on the 'relative allergic risk' of a number of proteins, defining an all-encompasing allergenicity hierarchy is not straightforward because sensitization to specific allergens can be contextual. During the first three years of life, most allergies that develop are to food proteins, whereas the prevalence of environmental allergen sensitization increases after this time[97, 198]. However, this does not preclude allergies to foods developing later in life, as they can form part of a normal diet during infancy but become problematic after cross-sensitization to an environmental allergen. This frequently occurs with class 2 fruit allergens, to which a large number of people are only sensitised after allergies develop to birch pollen during the teen years[187]. For example, exposure to Bet v 1 from birch can sensitize individuals to Mal d 1 in apple[186, 187, 199, 200]. Chronic workplace allergen exposure can also result in adult sensitisation, for example, in woodmill workers, fish packers, and bakers continually exposed to wheat flour[201-203]. Healthcare workers that regularly use latex gloves are similarly at increased risk of developing cross-reactive latex allergies than the general populace[76, 204].

Recently a unique subtype of IgE-mediated allergy has also been identified. The carbohydrate antigen galactose-alpha-1,3-galactose induces allergic responses to meats in adults[205]. This carbohydrate antigen is present on a number of mammalian meats, and results in an allergic phenotype presenting three to six hours after eating meat. This is thought to be because the meat needs to be digested to trigger mast cell degranulation. The symptoms of this type of allergy, contrasting with many allergies, involve the organ systems of the intestine, skin and sometimes systemic anaphylactic responses, but are not associated with allergic asthma[206]. This adult-onset allergy has also been shown to be caused by IgE cross-reactivity with Amblyomma americanum ticks, and to occur almost exclusively in tick-sensitized individuals[207]. Galactose-alpha-1,3-galactose also causes cross-reactions with a monoclonal antibody (the specificity of which is epidermal growth factor receptor[208], but this is irrelevant for the reaction) that contains the galactose-alpha-1,3-galactose sugar moeity in its 
heavy chain[209, 210]. While these findings also suggest that tolerance can be broken in special circumstances, it is clear that the sensitising capacity and aetiology of allergy is different for different proteins and foods. The mechanisms that explain these differences, and why only some people develop allergies to allergenic substances are only now becoming apparent.

\subsubsection{Physical and immunological differences in cow and goat milks}

Whole milk is a complex mixture of protein, lactose, oligosaccharides, a range of fatty acids, lipids, non-protein nitrogen, vitamins and minerals[211-218]. The protein comprises of two main fractions: The casein fraction and the whey fraction. Casein was originally defined by its precipitation at $\mathrm{pH}=4.6$ at $20^{\circ} \mathrm{C}[211]$, whereas the whey proteins remain soluble. There is significant species-to-species variation in the absolute composition of milk (Table 2). Different to other mammals, humans consume the milk of other species. For example, cow, goat and sheep milks are frequently included as part of the normal infant diet in different parts of the world[219, 220]. However, the consumption of milk from other species allows the possibility of allergy development to other species' milks. Allergies to cow, goat and sheep milk have all been reported, and milk allergies are not specific to western countries[34, 219, 221].

Breast milk is considered the gold standard in infant nutrition[222]. It is also important for the physiological development and maturation of the infant immune system (for review and discussion see[223, 224]). The main proteins in human milk are $\beta$-casein, $\kappa$-casein, $\alpha$-lactalbumin, lactoferrin, immunoglobulins, serum albumin and secretory component $[193,225,226]$. Approximately $60 \%$ of the total protein content of human milk comprises the more soluble whey proteins[227]. $\beta$-casein is the dominant human milk protein, followed by $\alpha$-lactalbumin[193]. The protein $\alpha$ s1-casein is present at low-to-undetectable concentrations[193, 228]. There are also a number of other trace proteins[229].

There are some significant differences in the composition of human and cow milks. In contrast to human milk, $80 \%$ of the protein in cow milk is casein[169, 230]. The 
casein fraction in cow milk comprises $40-50 \%$ s 1 casein plus three other casein proteins: $\alpha$ s-casein, $\kappa$-casein and $\beta$-casein $[169,211]$. The other $20 \%$ of cow milk protein is the more soluble whey fraction. The predominant whey protein is BLG (60 - 80\% of whey), with lower amounts of $\alpha$-lactalbumin, immunoglobulins, serum albumin and traces of lactoferrin[169, 211, 231]. In comparison to human milk, cow milk has additional proteins ( $\alpha$ s2-casein and $\beta$-lactoglobulin[193, 228]); a different whey to casein ratio; less non-protein nitrogen[223] and more concentrated protein, at $3 \mathrm{~g} / 100 \mathrm{~mL}[169,211]$ compared to approximately $1.6 \mathrm{~g}$ in colostrum, and $\approx 1 \mathrm{~g} / 100$ $\mathrm{mL}$ in at later times for human milk[223, 225].

For cow and goat milk, there are some differences and similarites in their composition. Cow and goat milks have similar total protein content, similar $\beta$-lactoglobulin content, and similar casein:whey balances[141, 169, 211, 231-235]. However, goat milk has lower levels of $\alpha$ s1-casein and higher $\beta$-casein levels[232, 234-236]. Like human milk, $\beta$-casein is the dominant protein in goat milk[233, 237]. One of the other differences between cows and goats, is that some breeds of goat produce milk with higher total fat content, and goat milk invariably has higher medium-chain fatty acid content than cow milk[235, 237]. The increased medium-chain fatty acid content renders goat milk fats more digestible by lipase activity[237]. Goat milk proteins are also more heatlabile[238] and more digestible by human- and simulated-gastric fluids than those of cow milk[185]. There are also differences in non-protein nitrogen content and composition between the two milks[215]. Mineral and vitamin content can differ between the species, but there is significant seasonal and study-to-study variation (c.f.[217, 235, 239]). The reported differences between cow and goat milks relate primarily to protein homology, $\alpha$ s1 casein content and the amount of medium-chain fatty acids present in the milks.

When individual proteins are examined, goat and cow milk proteins are more similar than cow and human homologues. Any given goat milk protein shares 85-95\% homology with its cow milk counterpart[169]. In humans, the most conserved protein, $\alpha$-lactalbumin, shares $76-78 \%$ sequence identity and almost $90 \%$ homology with cow $\alpha$-lactalbumin $[169,193,240]$, but other proteins are less homologous. Bovine and human $\beta$-casein are $47-57 \%$ homologous $[169,196]$. Human $\alpha$-s1 casein shares only partial $(\approx 32 \%)$ sequence homology with cow $\alpha$-s1 casein[241, 242]. While it is absent 
from human milk[228], BLG shares structural homology with serum retinol binding protein[177, 243]. It is also related to other human lipocalins, the glycodelin precursor protein[244], and human placental protein-14[245](44\% by DELTA-BLAST; see methods section). Allergens often share sequence similarity with host proteins[246], and the similarities in cow and human milk proteins may contribute to the frequent incidence of cow milk allergy in humans[246]. 
Table 2: Composition and homology of human, goat and cow milk

\begin{tabular}{|l|l|l|l|}
\hline & Human & Goat & Cow \\
\hline Protein $(\%)$ & $0.8-1.25$ & 4.3 & $3.2-3.6$ \\
\hline Caseins $(\%)$ & $\approx 40$ & $\approx 80$ & $\approx 80$ \\
\hline Whey $(\%)$ & $\approx 60$ & $\approx 20$ & $\approx 20$ \\
\hline Dominant milk protein & $\beta$-casein & beta-casein & $\alpha$ s1-casein \\
\hline$\alpha$ s1-casein & $0.8 \mathrm{~g} / \mathrm{L}$ & $0-3.5 \mathrm{~g} / \mathrm{L}$ & $12-15 \mathrm{~g} / \mathrm{L}$ \\
\hline$\alpha$ s2-casein & Not present & $3.8-3.9 \mathrm{~g} / \mathrm{L}$ & $3-4 \mathrm{~g} / \mathrm{L}$ \\
\hline$\beta$-lactoglobulin & Not present & $2.9-3.4 \mathrm{~g} / \mathrm{L}$ & $2-4 \mathrm{~g} / \mathrm{L}$ \\
\hline Homology $(\%$ to cow) & & & \\
\hline$\alpha$ s1-casein & 31.9 & 87.9 & 100 \\
\hline$\alpha$ s2-casein & N/A & 88.3 & 100 \\
\hline$\beta$-casein & 56.5 & 91.1 & 100 \\
\hline$\beta$-lactoglobulin & N/A & 94.4 & 100 \\
\hline
\end{tabular}

Proportions and concentrations are compiled from [141, 169, 193, 211, 225, 227, 228, 233, 235-237]; Homology data are taken from [169]. 
Evidence suggests that while goat and cow milk proteins are largely homologous, they have distinct properties as allergens. In one study, some cow milk allergic individuals tolerated goat milk, and in those that did not, as much as five times more goat milk was required to cause an allergic response compared with cow milk[192]. Further, the IgE-binding repertoire of sera from cow milk-allergic individuals generally binds to a comparably reduced set of goat milk proteins[192, 247, 248]. In terms of its ability to sensitize and evoke an allergic response in orally-sensitized mice, goat milk is less allergenic than cow milk[234]. Oral feeding of goat milk to guinea pigs results in less frequent challenge-induced anaphylaxis than cow milk[141]. Further, the allergenicity of ruminant milk correlates closely with its $\alpha$ s1 casein content, both in terms of IgE cross-reactivity in sera from cow milk allergic individuals, and in terms of its sensitizing and allergic response-evoking capacity in experimental systems[141, 232, 234, 236, 249]. Low $\alpha$ s1-casein levels are more typical of goat milks and demonstrate reduced allergenicity in mice and guinea pigs[233, 236, 249, 250]. Evaluating whether these differences can be attributed to differences in intrinsic allergenicity, or more predominantly to digestibility of the milk, remains to be addressed.

\section{$1.4 \quad$ Predicting allergenicity}

\subsubsection{In silico prediction}

As well as understanding the epidemiology of food allergy, predicting allergenicity and cross-reactivity are becoming more important in the modern world. Novel proteins are being introduced into the food chain, such as those from genetically-modified crops, and these need to be assessed for their allergic potential. Generally, crossreactive allergens share more than $70 \%$ sequence homology with each other[154], so in silico comparisons to known allergens can be used as an early step in the screening process (e.g., [251, 252]). In fact, the second step in the World Health Organisation decision tree for the evaluation of allergic potential, after assessing whether a protein is derived from an allergenic source, is to examine sequence homology using protein databases[253, 254] . However, in silico analyses are only a start point. Even if allergenic potential is not identified in structural screens, serum screens, animal models 
and screening for pepsin resistance are recommended by the guidelines[253, 254]. If a protein is able to sensitize animals, this is considered a significant risk factor for it to be a sensitizing allergen in humans[253, 254]. In silico screens also have the potential to reveal features of allergens of biological importance that may be missed in specific bioassays[152]. Allergenicity screening is beginning to incorporate in silico methodologies as an adjunct to biological testing (e.g., [255]).

\subsubsection{Biological evaluation of allergenicity and cross-reactivity}

Examining allergenicity in biological systems utilises a variety of approaches. Classical analyses have examined peptide recognition of sequential epitopes, and in some cases these have identified certain B cell epitopes that correlate with allergies that persist (e.g., $[256,257])$. Other approaches use surface plasmon resonance to examine the affinity and/or avidity of antibody for antigen, and this has been used to analyse $\operatorname{IgE}$ affinity distributions in polyclonal sera in some cases[258, 259]. Linear epitope analysis can be informative, particularly for $\mathrm{T}$ cell epitope detection, because $\mathrm{T}$ cells recognise short linear epitopes (generally 8-20mers); there are a significant number of allergens for which discontinuous B cell epitopes are thought to be key[260]. This is exemplified nicely in the study by Clement et al.[259], in which only 1 of more than 50 monoclonal antibodies recognised denatured BLG, but epitope masking was able to identify 12 antigenic clusters on the surface of native BLG[259]. When the first crystal structure of an IgE-bound allergen was solved, specific surface-exposed residues identified in the screen by Clement et al.[259] were also involved in IgE-binding to BLG[261]. Similarly, the majority of IgE binding to recombinant $\alpha$-lactalbumin is predicted to bind to a cluster of surface-exposed residues[262]. Combining structural data with epitope analysis has been useful in the indentification of discontinuous epitopes that would not necessarily be observed if the full structure of the protein was not examined.

A range of novel in vitro and in vivo animal methods for testing changes in the allergic potential of foods are being trialed. These include oral sensitisation using allergen in the presence or cholera toxin, followed by analysis of the T cell, B cell and allergen-induced mast cell response[263]. Measuring degranulation products of mast 
cells, the antibody response and the impact of feeding on basophil populations has been examined in this system to evaluate changes in the allergenicity of hydrolysates in mice[264]. It has also been used to compare the epitope specificity of $\operatorname{IgE}$ generated in mice to the epitopes involved in human $\operatorname{IgE}$ binding[265], and similarly to examine specific allergens contained in whole foods against which antibody responses are directed[263]. Similar examinations have examined the proteins to which antibody binds in guinea pigs sensitized to whole milks[232, 249]. There are numerous mouse models that examine a range of different aspects of the allergic process. I site some examples for further reading[136, 266-269]. In a similar attempt to clarify what makes chemical a (type IV) contact allergen, Kimber et al.[270] examine the response in an isolated lymph node assay[270]. In an independently derived, but similar assay, our lab has developed a novel mouse assay of allergenicity (see next section).

Collectively, a range of methods have been, or are being applied to the evaluation of the allergenicity of foods. These assays are also used to improve our understanding of the properties of allergens that render them allergenic and/or cross-reactive. Further, these models can provide information about the basic mechanisms that control the allergic process. Employing multiple biological approaches, in addition to in silico prediction methodologies will continue to be key for the determination of the allergenic potential of foods. 


\subsubsection{Development of a novel assay of allergenicity and Th2 function}

In addition to the various tools that have been developed to examine allergenicity, our lab has used the G4/+ reporter in an assay that measures allergenicity in direct proportion with Th2 cell development. In early studies by Belkaid et al.[271], intradermal administration with $L$. major promastigotes induced local inflammation isolated to the ear[271]. Our lab examined Th2 cell development induced by dead parasite antigens, known allergens, or bacterial products in the auricular (superficial parotid) lymph node[ $[1,57,272]$. In experiments using indian violet ink, our lab determined that this is the only lymph node to which antigen administered to the ear is drained (figure 1.2; photo and observation made by Graham Le Gros). By combining this sensitive, single lymph node assay with an IL-4-reporter mouse[273] IL-4-producing CD4+ T cells can be sensitively identified and counted. The inflammation can be used to examine immunogenicity and GFP $+\mathrm{CD} 4+\mathrm{Th} 2$ cell numbers can be used to compare allergenicity. Numerous immunogens have been compared in this system (figure 1.3; data generated by Mali Camberis and Melanie Prout). The most potent Th2-polarising immunogen that has been observed in this system, so far, is antigen derived from dead $i$ L3 N. brasiliensis larvae (figure 1.3). Dermatophagoides pteronyssinus house dust mite extract (HDM), also induces strong Th2 cell development (figure 1.3). The expansion of IL-4-producing Th2 cells, as reported by GFP expression in G4/+ mice is reproducible. In addition to the data shown (figure 1.3), my studies have recapitulated these original findings: Immunisation with dead parasite antigens, caused a 200-fold increase in Th2 cell numbers, and HDM generated four-fold fewer Th2 cells than dead $N$. brasiliensis larvae (figure 1.4B). In this manner, in vivo Th2 cell development can be examined and has the potential to be applied in the analysis of intrinsic allergenicity. 


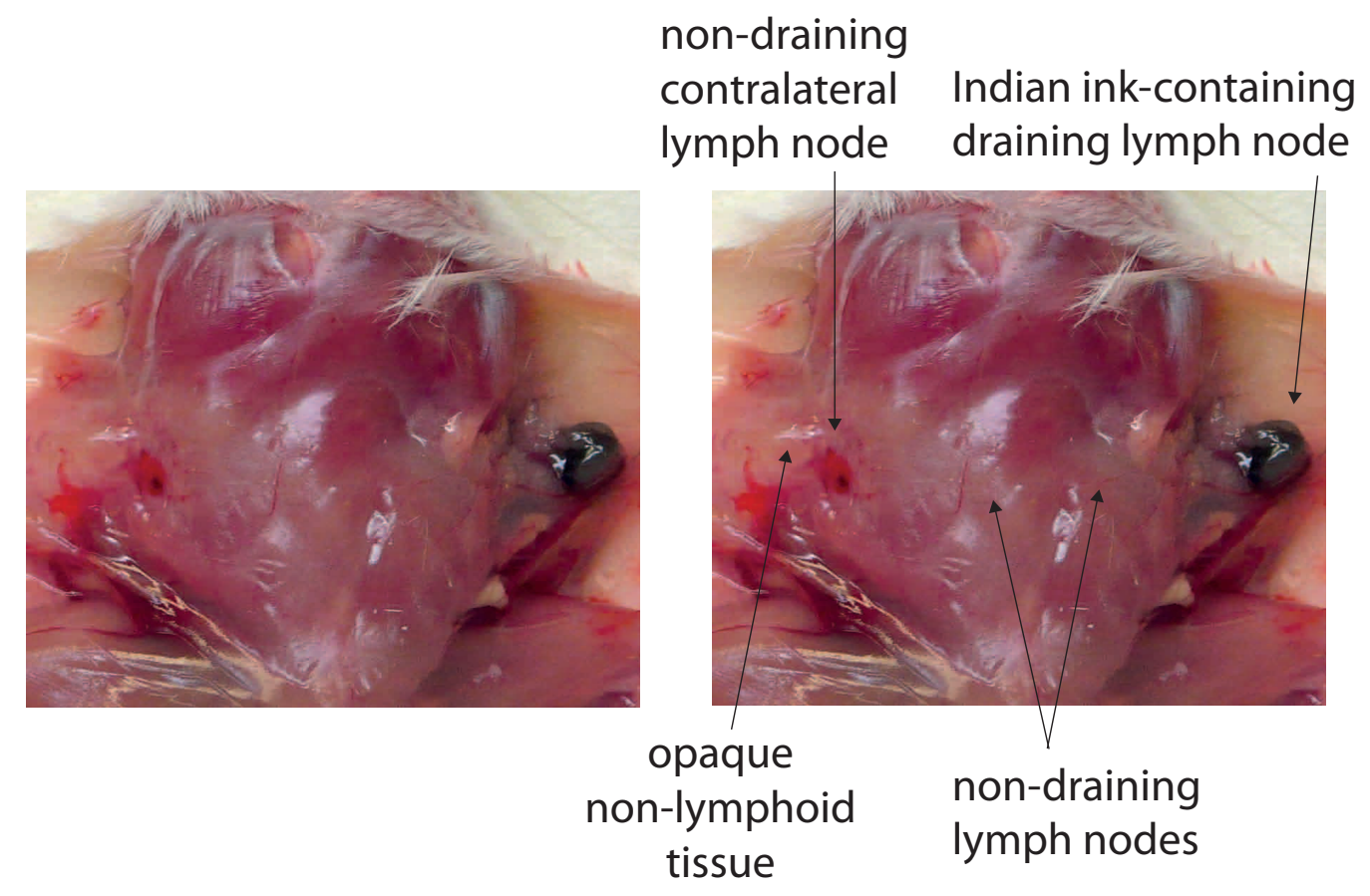

Figure 1.2: Indian violet ink drains to a single lymph node. Seven days after intradermal injection of 600 dead $i$ L3 N. brasiliensis larvae mixed with indian violet ink, the tissue architecture around the head of a mouse was photographed. The same picture is shown twice, and in the right-hand photo the location of the auricular draining (superficial parotid[272]) and non-draining (mandibular and accessory mandibular[272]) lymph nodes are indicated. In all other experiments, indian ink was not included in the injection. A non-lymphoid, likely adipose, tissue deposit is also indicated for disambiguation. The contralateral auricular lymph node can be seen at the arrow tip just above the ensanguined area at the junction of the superficial temporal and maxillary veins. 


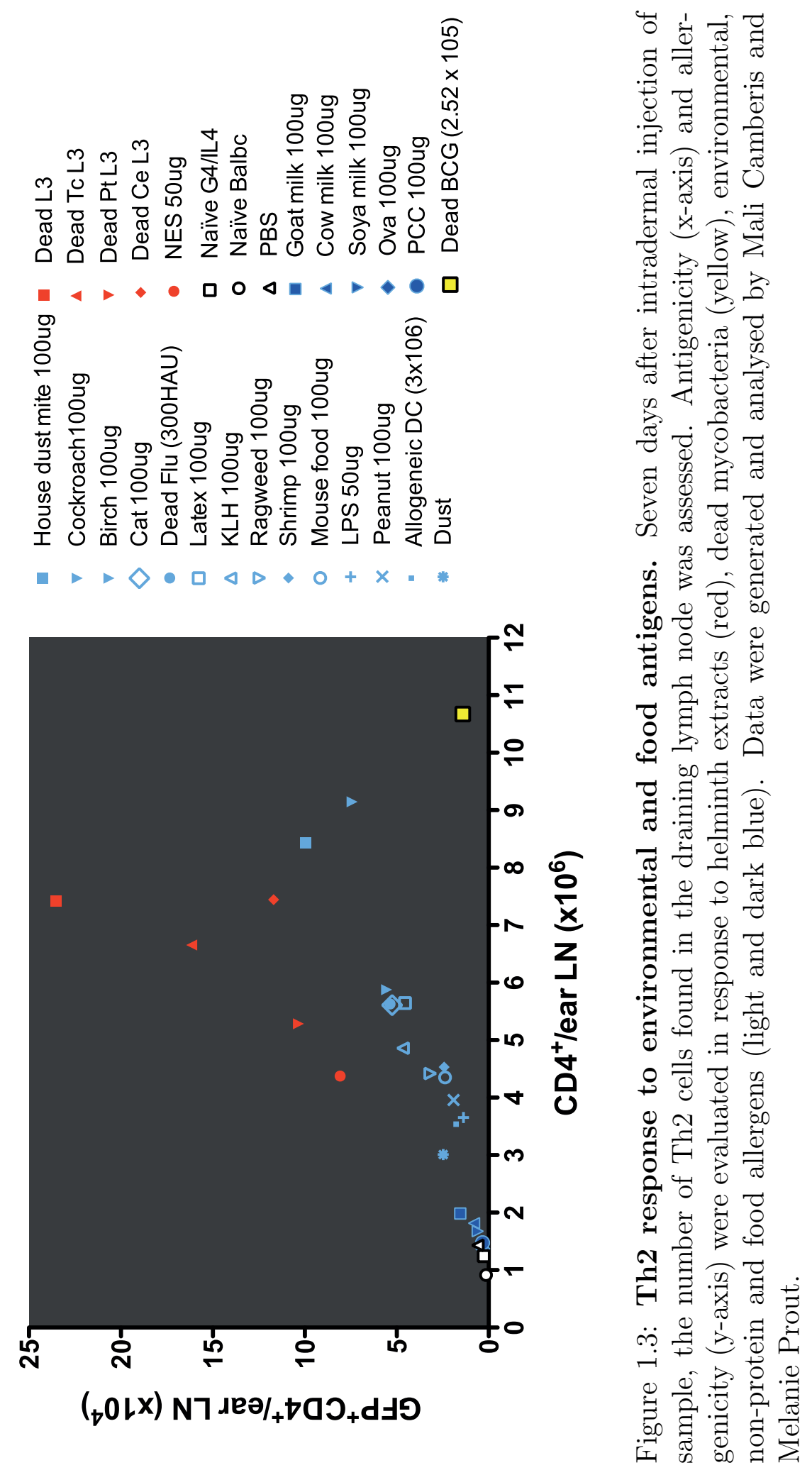



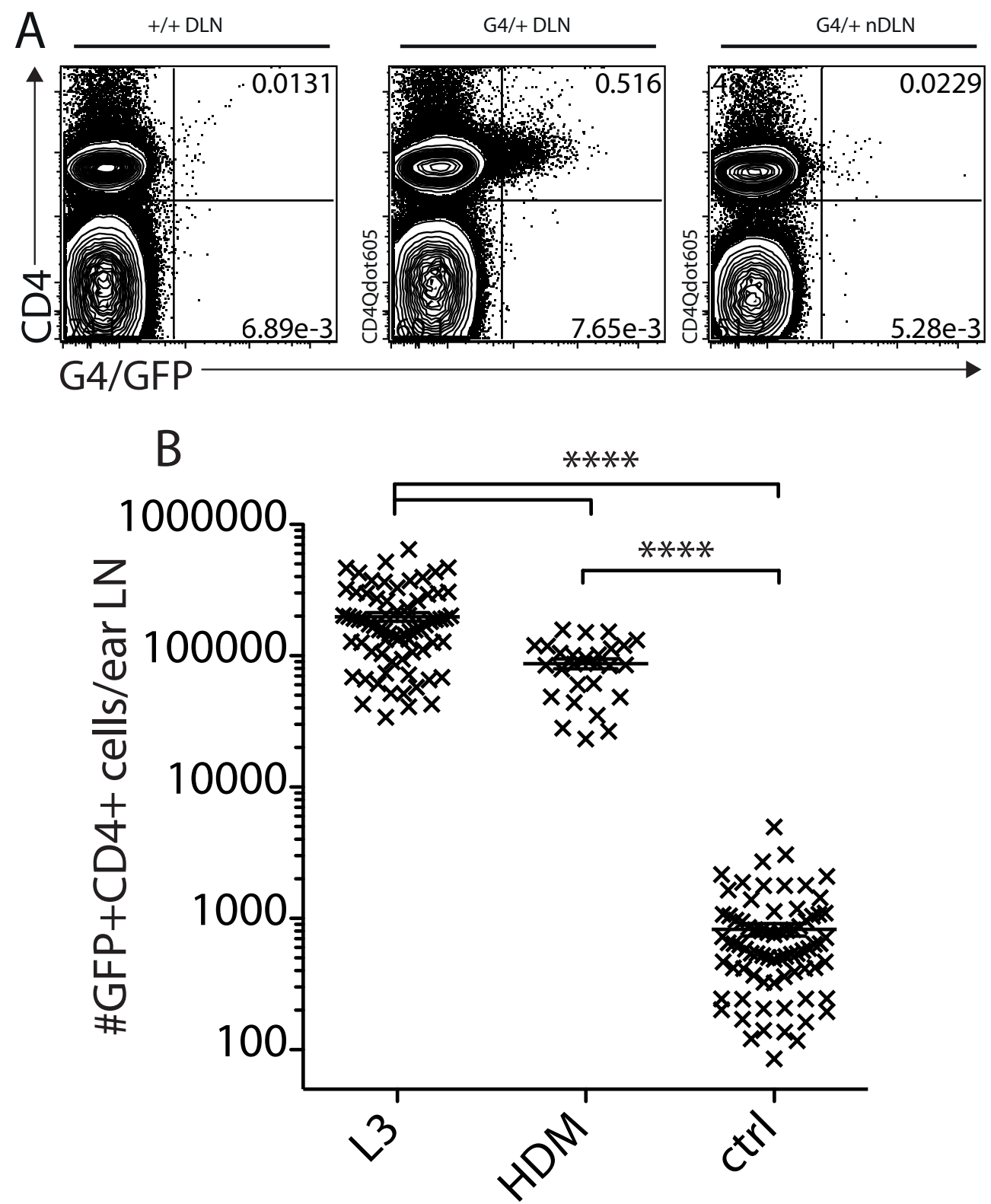

Figure 1.4: Th2 response to parasite and dust mite antigens. (A) Total live cells were examined by flow cytometry for CD4 and GFP expression in draining (DLN) and non-draining $(\mathrm{nDLN})$ lymph nodes of $+/+$ and $\mathrm{G} 4 /+$ mice. (B) The total number of GFP $+\mathrm{CD} 4+\mathrm{T}$ cells induced by immunization with 600 dead $i$ L3 N. brasiliensis larvae (L3) or $100 \mu \mathrm{g}$ HDM (HDM) in G4/+ mice were combined from all experiments described in this thesis hereafter. The control (ctrl) group includes naive, PBS-injected and non-draining nodes of G4/+ mice. Symbols show individual responses of $(n=72)$ N.b.-immunised, $(\mathrm{n}=27)$ HDM-immunised, and $(\mathrm{n}=76)$ control lymph nodes. Data were analysed by One-way ANOVA with Tukey's post-test. ${ }^{* * * *} \mathrm{p}<0.0001$. 


\subsection{The immunological mechanisms of allergic disease}

\subsubsection{The generation of IgE}

$\operatorname{IgE}$ is central to the allergic response, therefore, the processes that lead to IgE production are a key process in the allergen-directed immune response. IgE class switching is CD4+ T cell dependent[274, 275]. With some exceptions, isotype switching occurs in lymph nodes concomittant with affinity maturation[276-280]. The enzyme Activation-Induced Cytidine Deaminase is central to both processes[281]. Prior to isotype class switching, naive $\mathrm{B}$ cells excise the $\mu$ and $\delta$ heavy chains and begin to transcribe downstream heavy chain transcripts $(\gamma, \epsilon$ or $\alpha)[282,283]$. This means that naive $\mathrm{B}$ cells are $\operatorname{IgM}+\operatorname{IgD}+$, while isotype class-switched B cells will lose IgM and $\operatorname{Ig} \mathrm{D}$ expression and express $\operatorname{IgG}, \operatorname{Ig} \mathrm{E}$ or $\operatorname{Ig} \mathrm{A}$. When B cells mature into plasma cells, the class-switched B cell receptor is secreted as antibody of the class-switched isotype (for an example see [284]).

The cues required for B cell isotype switching to IgE have been determined and IL-4 is the most critical factor. In the 1980s it was discovered that when the protein BCF1, now known as IL-4, was added to cultures of mitogen-activated B cells or human peripheral blood B cells they class-switched to produce antibodies of the IgE and IgG1 isotypes (or IgE and IgG4 in humans)[285, 286]. In mouse studies, it was shown that blocking IL-4 signaling by sequestering secreted IL-4[287] or by blocking IL-4 receptor signaling[288], IgE production was impaired by greater than 95 percent. The advent of IL-4 knockout mice confirmed the central role of IL-4 in IgE production[289]. In response to infection with the rodent helminth $N$. brasiliensis that usually generates high titres of $\operatorname{IgE}$ and IgG1, IL-4-deficient mice generated no detectable IgE and exhibited an 80 percent defect in IgG1 production[289]. The cellular source of IL-4 that provokes IgE production is known to be the CD4+ T cell[274, 275] and CD4+ $\mathrm{T}$ cell expression of CD40-ligand (L) is also critical for the CD4-dependent isotype class switch[290-292]. Thus, by producing IL-4 and expressing CD40L, CD4+ T cells promote IgE class switching. 


\subsubsection{Additional roles of IL-4}

IL-4 influences not only IgE production, but also a number of other aspects of the allergic process. IL-4 increases mast cell FceRI-expression[293] and potentiates nonhaematopoeitic cells to respond to vasoactive mediators of allergy[48, 50]. IL-4 signaling is required for experimental allergic airways disease[294] and for oral allergeninduced mastocytosis that follows the first few oral challenges of sensitized mice[295]. Clinically, IL-4 and T helper type 2(Th2)-associated cytokine mRNA trancript numbers are elevated in bronchoalveolar lavage lymphocytes of asthmatics and in the jejunal tissue of food allergic individuals[90, 296] indicating that IL-4 may evoke local tissue effects during allergic and asthmatic responses. These Th2-associated cytokines include IL-4, and also IL-5 and IL-13. All three of these cytokines are observed at increased levels following oral allergen challenge in sensitized mice[37, 58].

\subsubsection{Mice are rendered susceptible to intestinally-triggered allergic responses in a post-sensitization oral potentia- tion phase}

The elevation in Th2-associated cytokines is an important aspect of the intestinal allergic process. While experimental intestinal allergic responses are IgE- and mast cell-dependent, after sensitization, multiple oral exposures are usually required before severe diarrhoea is observed; it is in this phase that precedes disease in which Th2 cytokines first become elevated[37]. In sensitized wild-type BALB/c mice, multiple oral challenges are required to generate sufficient intestinal mast cell infiltration to permit oral allergen-induced diarrhoea development[36, 37, 67]. Further, passive sensitization does not permit diarrhoea without multiple previous oral challenges[67]. It has, however, been demonstrated that once mice have been orally challenged multiple times with OVA, passive sensitization with IgE against the trinitrophenyl (TNP) hapten permits diarrhoea development upon the first feeding of TNPylated-bovine serum albumin[67]. This suggests that both peripheral sensitization and intestinal mast cell infiltration are required, but these are discrete and likely independent processes that permit allergic diarrhea to be triggered by oral allergen challenge. As this local Th2- 
type inflammatory process precedes observable diarrhea, I will refer to this process as oral potentiation.

IL-4 is key to oral potentiation, but other Th2-associated cytokines also become elevated during oral potentiation. In orally-challenged mice, IL-5 levels become elevated and increased intestinal eosinophil influx is observed[37, 58, 297]. There is no role for eosinophils in IgE-mediated diarrhoea[37], but they are implicated in villus shortening in oral-allergen challenged mice[42]. While beyond the scope of this thesis, eosinophils are also involved in other IgE-associated diseases, like eosinophilic esophagitis[11]. In terms of disease-relevant Th2 cytokines, IL-13 levels also increase in orally challenged mice[295] and blockade of IL-13 signaling decreases disease incidence in mice without significantly impairing mast cell infiltration[295]. This may be due in part to the effects of IL-13 on the smooth muscle tissue itself[50, 66]. The CD4+ T cell-derived cytokine, IL-9, is also implicated in the expansion of intestinal mast cells[57]. IL-4, IL-5 and IL-13 can all be produced by CD4+ T cells, so in addition to their role in IgE generation, there are a number of additional allergic processes that are associated with CD4+ T cell activity, including the oral potentiation that permits triggering of intestinal anaphylaxis.

\subsubsection{CD4 $+\mathrm{T}$ cells are implicated in oral potentiation}

Studies in three similar systems implicate intestinally-localised CD4+ T cells in the oral potentiation process[37, 57, 58, 298]. Prior to diarrhoea onset, sequential intragastric OVA challenges induces proliferation of- and $\alpha 4 \beta 7$ expression on- OVA-specific CD4 + T cells[38], IL-4 production by intestinal CD4+ T cells[57, 58], and intestinal transcription of CCL20[299]. Additionally, adoptive transfer of in vitro restimulated OVA-specific CD4+ T cells from diarrhetic mice transfers disease susceptibility to naive mice[38]. As outlined previously, this oral potentiation period is also associated with increases in intestinal mastocytosis. The involvement of the IL-4/-13 STAT-6 signaling pathway in experimental food allergy is unequivocal[37, 57, 58, 295], however the distinct effects of CD4-derived IL-4 on IgE production and tissue-specific mast cell activity remain unknown. There is also evidence that after sensitization, CD4 $+\mathrm{T}$ cell recirculation is not required for disease to develop[299]. This indicates 
that either peripheral processes (like IgE generation), or intestinally-localised CD4+ $\mathrm{T}$ cells are sufficient for oral potentiation. It has also been demonstrated that after oral potentiation, disease ensues when IL-4 is neutralised[295]. The processes involved in oral potentiation and those that maintain disease susceptibility may therefore be mediated by distinct cytokines and different cell types. In a similar vein, cells other than CD4+ T cells, namely basophils, eosinophils, $i \mathrm{NK} \mathrm{T}$ cells, mast cells and $\mathrm{B}$ cells have been shown to produce IL-4, and could therefore contribute to the oral potentiation process[300-305]. The correlation between Th2 cell infiltration and IL-4 production implicate CD4+ Th2 cells in the allergic process, but whether they are required, or simply sufficient, for the induction of intestinal anaphylaxis in mice requires clarification. The involvement of $\mathrm{CD} 4+\mathrm{T}$ cells in disease maintenance is also unclear: addressing the role of CD4+ T cells in the maintenance of disease is required. Close inspection of the role of CD4+ $\mathrm{T}$ cells in the post-sensitization phase of food allergy may establish unknown subtleties of the allergic process that facilitate better long-term disease alleviation.

\subsubsection{Cytokine requirements for intestinal mastocytosis}

Mast cells are key effector cells in IgE-mediated allergies. However, there is evidence that allergic responses that are induced by challenge in different tissues are mediated by different effector molecules. For some responses, passive sensitization through the transfer of IgE-containing serum is sufficient to permit immediate, local, type I hypersensitivity reactions. For example, injection of purified antigen-specific IgE, or antigen-specific IgE-containing serum into the ears of mice is sufficient to permit acute mast cell-dependent type I responses in the ears of mice[43, 306]. Passive sensitization can also permit systemic anaphylaxis in mice[41, 48]. Passive sensitization is possible in humans, too. Intradermal injection of reaginic serum to non-sensitized humans, followed by oral allergen ingestion, induces wheal-and-flare reactions in the skin without intestinal disease manifestations[150]. In mice, passive sensitization alone does not permit severe intestinal allergic responses without the oral potentiation phase[67]. Therefore, while $\operatorname{IgE}$ alone is sufficient to permit cutaneous and systemic disease, additional cues are required to promote intestinal mast-cell dependent allergic ana- 
phylaxis.

There are a number of mast cell-active cytokines, and three of these are critically involved in inflammatory mast cell responses in mice. It is known that stem cell factor $(\mathrm{SCF})$ is required for the presence of mast cells in the intestine at baseline[307]. Mutations in the gene encoding cKit render mice mast cell-deficient, in intestinal, peritoneal, and cutaneous tissues[43, 307, 308]. In contrast, while mast cells and their activity can be observed in mice deficient in IL-3, IL-4, or IL-9[50, 57, 309, 310], these three cytokines amplify intestinal mast cell infiltration caused by parasite infection[311-313]. These are the three cytokines that are most important for inflammatory mast cell responses. Exogenous administration of these cytokines can also expand intestinal mast cell populations $[309,314]$. IL-3, is a particularly potent mastcell active cytokine, and can increase intestinal mast cell progenitor numbers in the presence of SCF to a greater extent than IL-4 and IL-9 combined[314]. In vitro, IL-6 and IL-10 can also contribute to mast cell development[314]; however, each of IL-3, IL-4 and IL-9, has been demonstrated to expedite parasite expulsion and are therefore important in intestinal Th2 responses[50, 309, 311-313, 315]. Further, production of IL-3, IL-4 and IL-9 can be attributed, at least in part, to CD4+ T cell activity[316318]. Roles for IL-4 and IL-9 in experimental oral allergen-induced mastocytosis and diarrhoea have been demonstrated $[57,295]$ but the potential involvement of IL-3 in the intestinal allergic process is currently unknown.

\subsubsection{Intestinal mastocytosis heightens allergic disease sus- ceptibility}

Intestinal mast cells also contribute to additional aspects of the allergic disease process. High basal mast cell activity increases gut permeability, and this renders mice more susceptible to allergic sensitization and anaphylaxis[57, 67]. This is associated with a corollary decrease in blood plasma volume and impaired basal intestinal barrier function at baseline[57, 67]. Individuals with food allergies also have increased intestinal permeability, or a leaky gut[319]. Long-term changes in permeability are also observed in orally-primed wild-type mice[37, 57, 149]. It has also been demonstrated that hypothermia and shock can be induced by oral feeding of allergen, but only if 
the allergen can cross the intestinal epithelial barrier and get into the blood[67, 68]. Correlations have also been drawn between mast cell numbers and orally-induced anaphylactic severity[36, 39]. Intestinal uptake of allergen is expedited by sensitization in a mast-cell dependent manner[308, 320], and the allergic leaky gut results in a 200-fold increase in allergen levels in the blood of allergic mice fed allergen compared to non-allergic mice[149]. Oral allergen challenge has also been shown to enhance systemic anaphylactic responses[37] and exacerbate lung inflammation to unrelated allergens[321]. These findings collectively suggest that intestinal mast cell activity can exacerbate not only intestinal allergic responses, but can also permit allergen dissemination and amplify systemic allergic responses. Processes that increase intestinal mast cell activity therefore have the potential to promote allergic responses, and this may be an important aspect of the clinical allergic process. 


\subsection{Th2 cells and the functional Th paradigm}

While the full role of CD4+ T cells in allergic disease requires continued investigation, impetus already exists to investigate the signals that promote their development into Th2 polarised cells. Since 1986, IL-4 production by a CD4+ T cell has identified it as part of the Th2 subset, and much is now known about Th2 cell development in vitro; much still remains to be determined regarding their development in in vivo allergic disease contexts. In order to understand the early events that induce naive CD4+ T cells to attain Th2/IL-4-producing function the host-derived signals that lead to Th2 development have been investigated in detail.

The designation of the Th2 cell came, originally, from studies by Coffman and colleagues[322] in which CD4+ T cells were shown to develop into two mutually exclusive subsets based on function and their cytokine production profile. Long-term $\mathrm{T}$ cell clones that produced IL-2 and IFN-g were termed Th1 clones, whereas those that produced IL4 (then called BCF-1), IL-5 and IL-10, were designated Th2. Th2 clones were the only clones capable of enhancing IgE and IgG1 switching during polyclonal B cell stimulation[322] and Th2 clones also permitted mast cell generation (both of these responses were identified as IL-4-dependent processes[322]). Further, irrespective of the strain of the mouse used, immunization with chicken red blood cells gave rise only to Th1 clones; in contrast, Th2 clones exclusively were generated by fowl gammaglobulin immunization. Thus, from the first demonstration of Th subset polarization, the concept was inherently tied to that of protein allergenicity. While Th2 cells are implicated in allergic disease and anti-parasite responses[38, 57, 58, 323], Th1 cells are effector cells that are important for the control of intracellular pathogens[324]. There are now a number of proposed Th subsets, including Th9, Th17, Treg (regulatory) and TFH (follicular helper) cells, and multifunctional populations that do not segregate clearly into any one particular subset[324, 325], however, Th2 cells remain an important allergy-associated cell. The involvement of Th17 cells in some allergic processes have been gaining interest[158], and in terms of the oral potentiation process, IL-9 derived from Th9 cells may also play an important role[57, 326]. However, the central role of IL-4 in the allergic process means that IL-4-producing Th2 cells remain the focus of this discussion. 
If Th2 cells promote allergy, and allergenic immunizations promote their development, the question arises, what are the immunological changes triggered by an immunization that promote the differentiation of a CD4+ $\mathrm{T}$ cell toward any specific, and particularly a Th2 pathway? Commitment to a given Th subset is determined by lineage-specifying gene activation, and silencing of lineage-inappropriate cytokine genes[324, 327]. The master regulator of Th2 commitment, GATA-3[328] acts in conjuction with STAT-6 for the full elicitation of Th2 development and function in vivo[329-331], and simultaneously silences Th1 commitment[332]. T-bet is the master Th1 specifying transcription factor[333], and acts in collaboration with STAT-1[334]. Th17 cells are under the control of the master transcription factor ROR $\gamma \mathrm{t}[335]$, and are cross-antagonised by Foxp3 which specifies Treg development[336]. TFH cells, which are specialized B helper cells[275, 337, 338], are now known to develop under control of BCL-6[339-341]. In allergic disease IL-4, IL-5 and IL-13 producing Th2 cells are the most important Th subset and understanding the events that initiate and enhance their development are key current investigations.

\subsubsection{The early and late events specifying Th2 polarization in experimental systems}

The correlation between Th2 cells and allergic disease is clear, however, the events that permit their development remain incompletely defined. In addition to the role of the allergen in the induction of the allergic process, significant effort has gone into investigating the host-derived proteins that permit the acquisition of polarised $\mathrm{Th}$ function. Some work has been done in vivo, but the majority of understanding comes from in vitro studies.

In vitro studies have been important in elucidating the factors that promote Th2 cell development. Early studies identified IL-2 and IL-4 as important factors for in vitro Th2 cell differentiation[342-344]. Studies have now established that early IL-4 transcription is triggered by TCR ligation, CD4 co-receptor stimulation and IL-2Rsignaling, and only later is IL-4R signaling required for continued Th2 development [345349]. When naive CD4+ T cells are stimulated by plate-bound anti-CD3/CD28 or antigen presenting cells loaded with cognate antigen, increases in IL-4 mRNA can be 
detected as early as one hour after stimulation, but in the absence of IL-4R signaling this is not maintained, and Th2 cell development thereafter is impaired[347-349]. Similarly, studies using an IL-4-linked conditional toxic gene construct identified that naive CD4+ T cells will die upon initial stimulation, but if they are first polarized to the Th1 subset they will not die[327, 350]. This indicated that the IL-4 gene is transiently expressed by naive $\mathrm{T}$ cells, but an important part of differentiation is silencing of alternate lineage cytokines thereafter[327, 347, 350]. These studies identified that Th2 development and IL-4 expression in vitro begins in an IL-4-independent manner, and this is followed by a period in which IL-4 specifies Th2 lineage commitment[347]. The importance of this IL-4-independent phase of in vitro Th2 cell development is only beginning to be appreciated, but has lead to a number of investigations into the Th2-initiating processes. More recently, tissue-derived cytokines have been demonstrated to promote Th2 cell development by modulating dendritic cell function in vitro [351]. In addition to the cytokine milieu and involvement of IL-4 in Th2 development, TCR affinity also influences Th polarization[349] and increased cellular stress is associated with Th2 polarization[352].

The conditions that lead to Th2 cell development in vivo are more complex. There is evidence that demonstrates Th2 immunity is amplified by the innate-type cytokines IL-25, IL-33 and TSLP[353-356], but these cytokines are not required for the development or function of CD4+ Th2 cells[357-359]. The role of IL-4 in Th2 development in vivo shares similarities with the in vitro data but is less clearly understood and far less rigorously validated. While IL-4 can maintain the population of cells transcribing IL-4 in vivo[360-362], IL-4 signaling is not required for early Th2 generation $[1,360,362,363]$. Studies over the last 15 years have investigated the role of IL-4 in Th2 development in vivo and determined that the early generation of Th2 cells is equivalent in IL-4Ra-/- and WT mice[362]; memory Th2 numbers are similar in doubly IL-4 and STAT-6 deficient mice[1]; IL-4 mRNA accumulation is equivalent in early immune responses[360]; and naive IL-4Ra deficient CD4+ T cells that have received TCR ligation will produce IL-4 on ex vivo TCR stimulation[363]. However, when IL-4Ra deficient mice are injected in the hind footpad with OVA plus alum adjuvant, draining lymph node IL-4 transcription is induced, but transcript levels decay relative to wild-type mice concomitant with lymph node atrophy as the im- 
mune response progresses[360, 361]. Thus, IL-4 can influence the amount of IL-4 transcribed in an isolated lymph node in vivo, at least in an adjuvant based immunization regimen. Further, when IL-4 signaling is interrupted in mice responding to helminth infection, full Th2 effector function is diminished, as bronchoalveolar lavage (BAL) eosinophilia, which is a normal attribute of the anti-helminth response, and IgE switching are lost[1, 289], ex vivo T cell IL-4 production is curtailed[363], and the maintenance of Th2 cell and B cell populations is impaired[362]. It is becoming apparent that IL-4 is not required for Th2 development in vivo, but ascertaining the influence, rather than dependence, of IL-4 on Th2 cell development in primary Th2 contexts is becoming an increasingly important direction of current allergy research.

As such, studies investigating the role of IL-4 in allergen-induced Th2 development require further investigation. The in vivo studies that demonstrate a potential role for IL-4/STAT-6 signaling in Th2 development have examined mRNA transcript levels[1, $360,361]$ and more recently an mRNA-tagged IL-4 reporter[362] either in response to live parasite infection[1, 362] or following adjuvant-based immunization[360, 361]. Other studies of allergenicity in an adjuvant-free context have had to rely on the $\mathrm{B}$ cell response as the primary readout of Th2 development[268]. Understanding the involvement of IL-4 in in vivo Th2 development as a function of allergenicity is an important, but unexplored area. In particular, close dissection of the role of IL-4 in Th2 development, and the relationship between Th2 cells and the other identified in vivo IL-4-producing cell, the TFH cell, induced in an adjuvant-free allergic context may provide new insight into the aetiology and initiation of the allergic disease process.

\subsubsection{Are there multiple populations of IL-4-producing CD4+ $\mathrm{T}$ cells?}

In 2009, Reinhardt et al.[275] along with two other groups[362, 364], provided evidence that lymph node IL-4 derives predominantly from CD4+CXCR5+PD-1-hi TFH cells located in follicular regions of lymph nodes. These works followed the identification of TFH cells that have transcribed IL-4 mRNA during T-dependent B cell 
responses[337]. Subsequently it was shown that the molecule GL-7, which is expressed on activated T cells[365] and germinal centre B cells[338], demarcates germinal centre TFH that contain particularly high levels of IL-4 mRNA[366]. IL-4 producing Th2 cells that do not have the TFH phenotype or produce the hallmark TFH cytokine, IL21 , can also be found in the tissues[275, 317]. It is possible that these tissue-localised Th2 cells have come from the TFH cell pool[367], however, evidence is accumulating that lymph node versus tissue effector fate can be dictated early during lymph-node $\mathrm{T}$ cell activation[368, 369]. Additional data shows that IL-4 production by TFH is not strictly limited to Th2 immunisation, because IL-4 production by TFH cells is observed not only in response to Th2 stimuli such as live $N$. brasiliensis, H. polygyrus bacheri, L. major and S. mansoni infection[275, 362, 364], but also following lymocytic choriomeningitis virus (LCMV) infection[366] and in lymph node- but not lung-localised CD4+ T cells responding to experimental influenza virus infection[370]. Thus, while IL-4 production is typically associated with Th2 immunity and allergy, understanding the function and eventual fate of the developing IL-4-producing CD4+ $\mathrm{T}$ cell may have implications for the potential disease phenotype of a given immunisation strategy. 


\subsubsection{Measuring IL-4 production using IL-4 reporter mice}

One of the difficulties in monitoring Th2 development and IL-4 production in vivo is the short half-life of IL-4[371]. To facilitate better detection of IL-4 production, four different IL-4 reporter constructs have been reported in the literature[273, 372-374], and the use of three of these is still commonplace. The three widely used reporters are the 4get, the KN2 and the G4 reporter constructs (Figure 1.5). Each differs slightly in the way it has been inserted into the IL4 gene and they provide complementary, but context specific, information about the regulation of IL-4 production.

First, the 4get construct is an IL-4 mRNA tagged enhanced green fluorescent protein (GFP) construct that adds the sequence for GFP protein with an internal ribosomal entry site (IRES) construct to the 3' region of the gene encoding IL-4[372]. Thus, the 4get construct remains IL-4 sufficient, has the 5' untranscribed region (UTR) intact and at least $5 \mathrm{~kb}$ of the 3' UTR of the IL-4 gene remaining as part of the replaced gene segment[372]. The 4get construct remains IL-4 sufficient because the eGFP gene is placed directly downstream of the full-length IL-4 sequence. However, it has been shown that GFP expression in 4get cells does not demarcate cells actively producing IL-4, but rather identifies cells with the potential to produce IL-4[373], such that $\mathrm{GFP}+$ 4get cells are considered 'Th2 competent' cells[275]. The 4get construct is generally used in conjunction with the IL-4 knockin human CD2 (hCD2) construct, abbreviated to 'KN2'[373].

The KN2 reporter replaces the first two exons, plus the intronic region between exons 1 and 2, of the murine IL-4 gene with the gene encoding hCD2 without disrupting other IL-4 promoter/enhancer regions (see supplementary data of[373]). The murine CD4 enhancer is included in the construct to amplify the hCD2 signal to detectable levels[373, 375]. KN2/KN2 mice, homozygous for the KN2 allele, are IL-4 deficient[373]. However, when KN2 cells are provided with Th2 stimulation, cells will express hCD2 on the surface, and this can be detected by flow cytometry[352, 373]. The advantage of the KN2 reporter is that in in vitro $\mathrm{KN} 2 /+$ heterozygote Th2 cultures up to 80 percent of IL-4-producing cells express hCD2[373]. Further, Th2 immunizations that induce IL-4 production in vivo also induce hCD2 expression, and the longer half-life of hCD2 makes it simpler to detect IL-4 producing cells than using 
conventional methods[373]. However, not all hCD2+ cells express IL-4, so while the $\mathrm{KN} 2$ construct is a useful tool for studying IL-4 synthesis in vivo, there remain some nuances of this reporter construct that will need clarification in the future.

The G4 construct inserts the gene encoding (enhanced) GFP into the IL-4 gene, replacing exon 1 and 178 nucleotides of the first intronic region of $I L 4$ [273]. The position of the insertion renders the G4 allele IL-4 null, like the KN2 construct, such that G4/G4 homozygous mice lack IL-4-dependent features of Th2 immune responses[1]. The reporter mouse developed by Littman and colleagues[374] was similar to the KN2 reporter, but is no longer used.

The methods used to generate the knock-ins of all three reporters were similar. The Littman group originally designed the hCD2 construct for insertion into the CD4 gene[375], but by altering the target site they were able to insert the construct into the IL-4 gene but with low detection levels in Th2 cells[374], so they included the murine CD4 enhancer sequence with the hCD2 knockin, such that higher levels of hCD2 were observed in conditions under which IL-4 would normally be expressed[374]. The hCD2 protein was modified to render it non-functional when expressed, and is therefore considered to operate solely as a 'reporter' [375]. The construct also included a neomycin resistance cassette which was used to identify successfully incorporated transgenes. The KN2 construct used the same reporter construct, but with an additional lox-cre site included to remove the neomycin resistance cassette after positive selection. The G4 construct also contained a lox-cre neomycin resistance cassette but does not include the CD4 enhancer site. Mohrs et al. used transient cre recombinase electroporation[373], and $\mathrm{Hu}-\mathrm{Li}$ et al. crossed reporter mice to C57BL/6-cre mice in the F1[273] to remove the neomycin resistance cassette. The stem cells used for electroporation for the constructs were from strain 129, strain 129/SvJ, and E14 embryonic stem cells for the G4, KN2 and Littman constructs, respectively. All three were transferred to C57BL/6 blastocysts to yield chimeric mice, and then outbred for ten generations. Lastly, the G4 construct additionally contains the herpes simplex virus thymidine kinase gene 5' of the reporter, which allowed gancyclovir sensitivity screening assays to be used in the selection process. Both the G4 and KN2 reporters therefore were introduced in slightly different manners, and disrupt the IL-4 gene at different sites. 


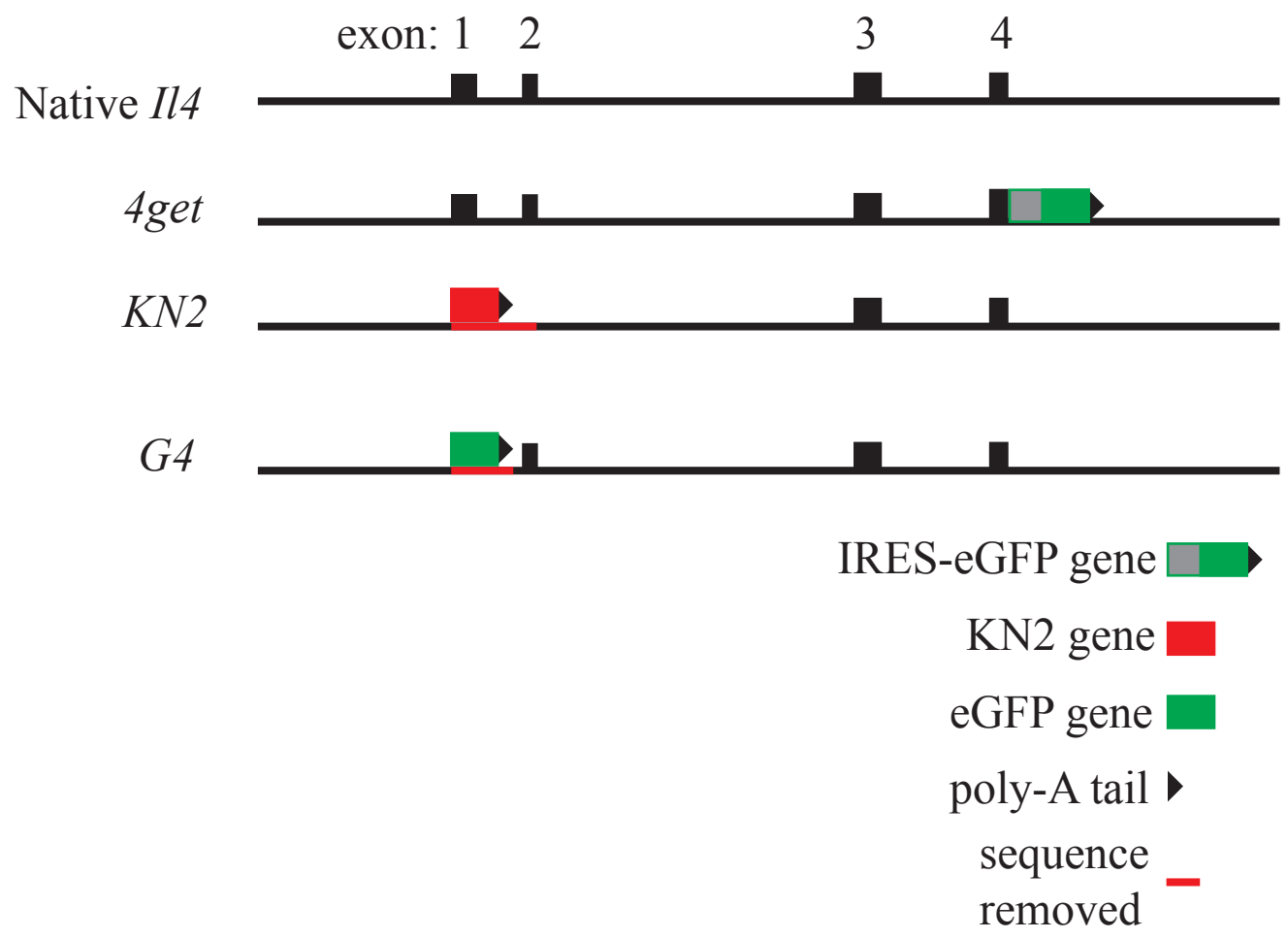

Figure 1.5: Insertion sites of 4 get, KN2 and G4 reporter constructs. (A) Wildtype $I l_{4}$, (B) Addition of the eGFP gene with an internal ribosomal entry site was inserted upstream of the polyadenylation site of the full-length IL-4 cDNA for the 4get construct. (C) The KN2 construct replaces exon 1, intron 1 and exon 2 of the murine Il4 gene. (D) The G4 construct replaces exon 1 and the first 178 nucleotides of intron 1 of the Il/4 gene. IL-4 protein is not produced from either the KN2 or the G4 allele. Data are modifed from [273, 372, 373, 375]. 


\subsubsection{The regulation of IL-4 production}

\section{Epigenetic changes influence cytokine gene accessibility}

The development of the polarised IL-4-producing Th2 cell phenotype is now established to involve many regulatory processes. With increasing proliferative cycles, Th2 cells become more likely to produce IL-4 and loose their capacity to produce IFN$\gamma[347,376]$. During this process, the chromatin structure of the Th1 genes becomes less accessible, and that around the Th2 locus becomes more accessible[347, 377]. This process involves changes to the chromatin structure that are the result of epigenetic modification.

Epigenetic modification is important in Th2 cell development. This is exemplified clearly in an early study by Bird et al.[378]. In this work, STAT-6-deficient CD4+ T cells cultured in IL-4 did not subsequently produce IL-4; however, if the cellular methylation/acetylation apparatus were interrupted, 16\% of STAT-6-deficient CD4+ T cells produced IL-4 upon restimulation[378]. Numerous studies have confirmed the importance of changes in gene access and chromatin modification to the development of the Th2 phenotype[377, 379-384]. Regulation of gene access is now recognised as an important part of Th2 regulation (For review, see[324, 385]).

\section{Probabilistic processes in biology}

In addition to epigenetic regulation, some biological processes occur through stochastic, or probabilistic mechanisms. The immune system is no exception. T cells activated under Th2-polarising conditions become increasingly likely to produce IL-4 with increased proliferative cycles[376] and are progressively more likely to inactivate Th1 genes with sequential divisions[347]. However, synchronously stimulated T cells do not divide synchronously: probabilistic regulation influences their rate of division and dictates the eventual Th cell phenotype that arises[376]. Probabilistic mechanisms have also been shown to underlie B cell population expansion, by balancing proliferation, isotype class-switching and death[386]. In this manner, 'chance' processes can 
affect the eventual biological response.

\section{Probabilistic gene expression}

Probabilistic regulation can account for the expression of some genes. Probabilistic regulation was proposed, based on the observation that in response to a given stimulus, many cells will produce a large amount of a gene product, and others will remain inactive[387]. A good example of this is seen in cells retrovirally transfected to express Lacz, rather than produce retrovirus: At baseline, very few cells will express $\beta$-galactosidase, reflecting Lacz activity, but those that do, exhibit significant activity[387]. The proportion of cells that exhibit activity increases when the 'signal strength', or exogenous stimuli that promote transcription increases. This is argued to be an attribute of an error-prone process, the success of which was increased by the multiplicative success of activation of enhancers[387]. In cases like this, the amount of a gene product produced by a clonal cell population will be controlled in an on/off manner, with cells producing either a lot, or no, product (figure 1.6B). This contrasts with the conventional, deterministic view, in which the 'signal strength' influences the transcriptional activity or amount of a gene product from each cell (figure 1.6A). In this manner, stochastic processes can regulate gene expression.

\section{Probabilistic regulation of IL-4 gene expression}

Probabilistic regulation of the Th2 locus has been proposed based on numerous studies identifying monoallelic expression of IL-4. When Th2 cells are activated in vitro, or expanded from clones, in a substantial proportion of cells, only one IL-4 allele will be detected. In the G4 reporter system, when in vitro-generated G4/+ Th cells are stimulated with antigen-loaded APCs in the presence of IL-4, approximately $7 \%$ will produce IL-4, approximately $7 \%$ will express GFP and less than $1 \%$ of cells will produce both IL-4 and GFP[1, 273, 388]. In other studies, IL-4 mRNA from only one allele was detected in approximately half of cloned Th2 cells[389]. It has also been observed that differences in IL-4 production between different strains of mice relates to the frequency of CD4 $+\mathrm{T}$ cells that produce IL-4, rather than regulation based on 
the amount produced by each cell[390]. These observations have lead to the proposal of probabilistic monoallelic regulation as an advanageous means by which to regulate IL-4 production[381].

However, some equivocality exists in the predominant expression profile and definitive determination of whether cytokine genes, including IL-4, are regulated at the single allele level[352, 373, 391]. While induced by simular stimuli as IL-4 protein and the G4 allele, the KN2 reporter operates differently to the G4 construct in one key aspect - Where G4 GFP expression occurs mutually exculsive with IL-4 protein in $80-90 \%$ of in vitro Th2 cells[1, 273, 388], hCD2 expression occurs in $80 \%$ of IL-4 producing KN2/4get Th2 cells in vitro[373]. Further, where approximately equivalent proportions of GFP and IL-4-producing cells have been observed soon after TCR restimulation of G4/+ cells $[1,273]$ and also at times when IL-4 mRNA levels are at their peak[388], in the KN2/4get system a greater proportion of Th2 cells express hCD2 than IL-4, such that $60 \%$ of hCD2+ cells do not produce IL-4 protein[373]. More recent studies have, however, shown a less complete dominance of hCD2 over IL-4 expression in KN2/+ cultures[392]. Together, these data indicate that our understanding of the regulation of IL-4 production is incomplete and requires further clarification, both in vitro and in vivo. 


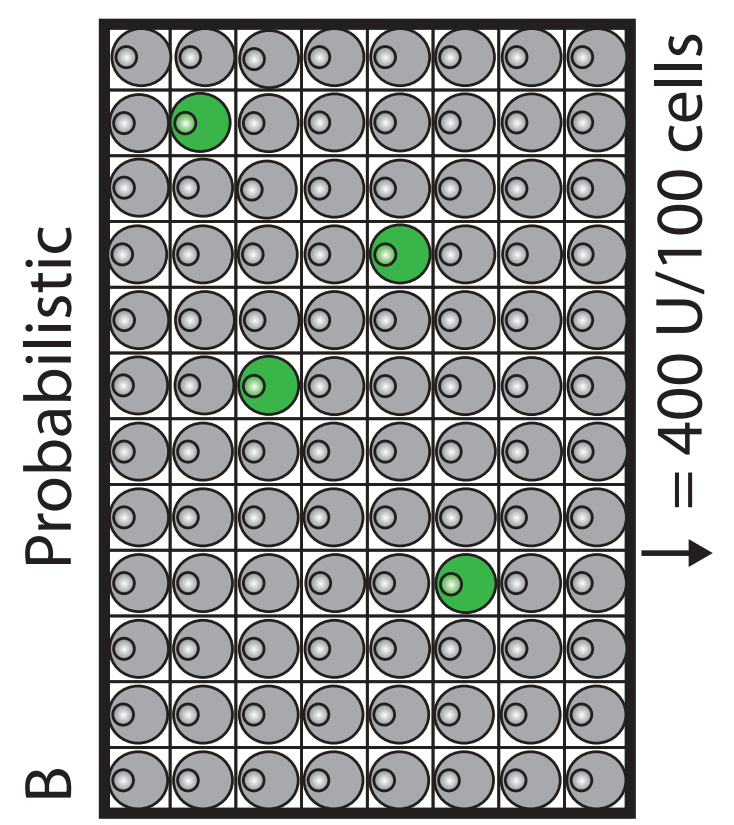

.ี 幽品

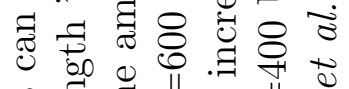

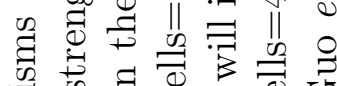

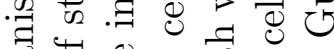

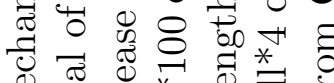
塻

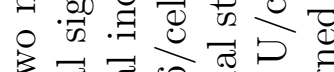
㻤预 ¿ี

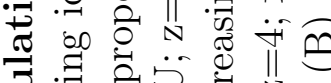

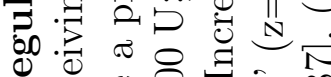

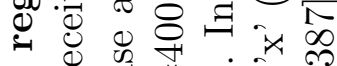

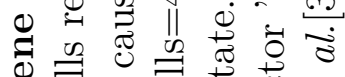

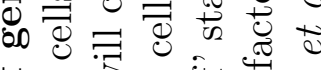
फै

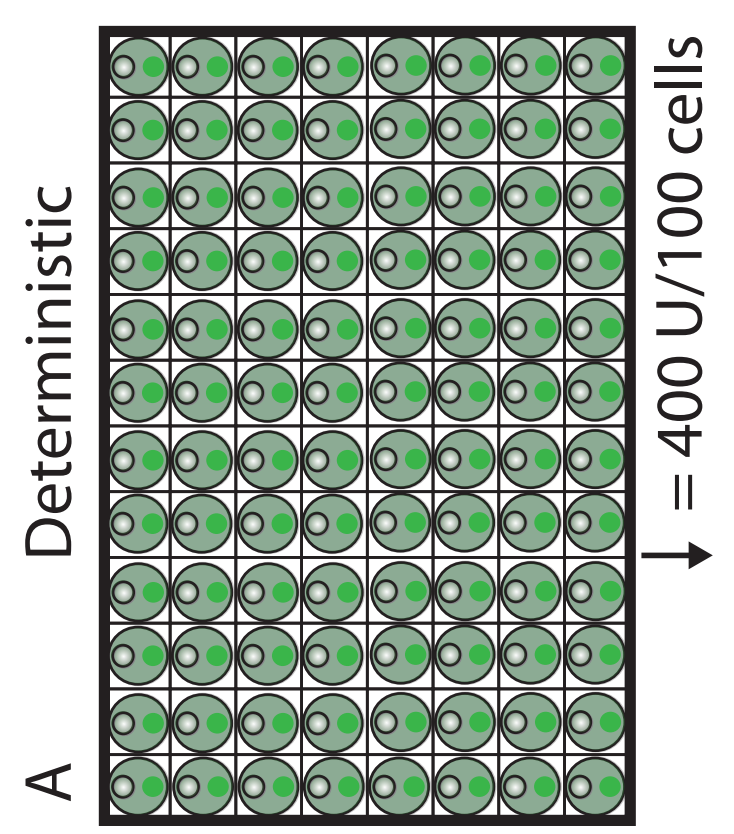

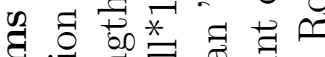

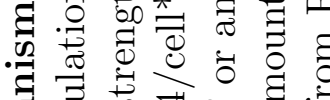

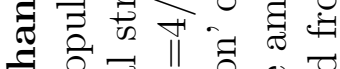

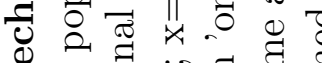

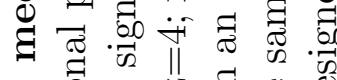

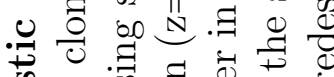
.0.0

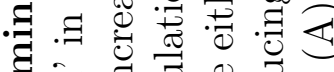

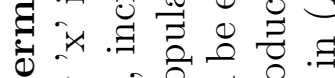

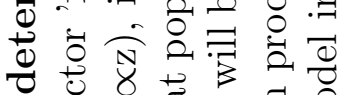

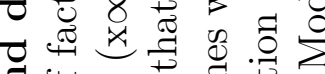
ส . :

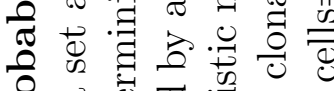

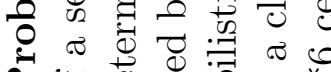

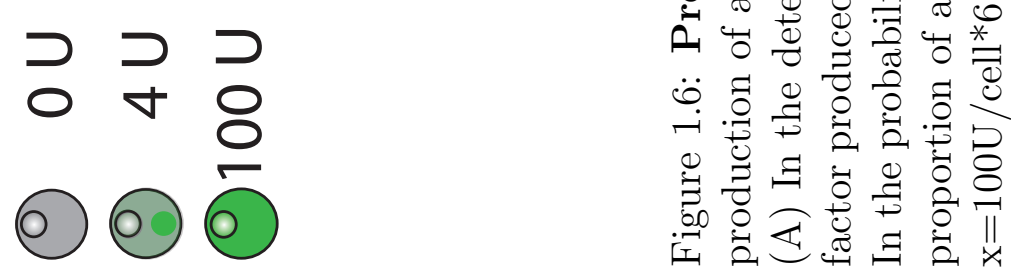




\subsection{Themes of this thesis}

The primary goal of this thesis was to clarify the key factors involved in the allergic process. This resulted in targeted investigations into the development and function of IL-4-producing CD4+ Th2 cells in allergic Th2 immune response models. Three questions pertaining to Th2 cell function and development were examined during this work, and relate, first to the controversial role of IL-4 in Th2 cell development in vivo; second, to their involvement in tissue-specific intestinal allergies; and third, to the way allergens initiate Th2 cell development and can be compared allergenically. After a short investigation characterising a novel reporter mouse used in our in vivo studies, these concepts form the focuses of chapters 4-6. The relevant background, and an overview of the concepts tested in each section are introduced at the beginning of each chapter.

The over-arching aims of this thesis are:

1) To clarify whether IL-4 alleles are regulated in an allele-specific, probabilistic monoallelic process in vitro;

2) To determine the role of IL-4 in in vivo allergen-induced primary Th2 cell development;

3) To examine the influence of IL-4 heterozygosity on Th2-dependent allergic immunity;

4) To clarify the role of CD4+ T cells in the oral potentiation of allergic disease;

5) To determine whether there is an immunological basis to the differential allergenicity of cow and goat milk components. 



\section{Chapter 2}

\section{Methods}

\subsection{Special reagents and miscellaneous procedures}

\subsubsection{Allergens}

House dust mite (HDM) was purchased from Greer laboratories (Lenoir, NC, USA). Albumin from chicken egg white (OVA) was purchased from Sigma (St Louis, MO, USA). Bovine serum albumin (BSA) was purchased from Sigma. iL3 Nippostrongylus brasiliensis larvae were cultured in house according to established protocols[393] and were killed by three freeze-thaw cycles at $-80^{\circ} \mathrm{C}$. The whole casein fraction from cow and goat milks were prepared as described[394] and were provided by Ali Hodgkinson, AgResearch, Ruakura. Briefly, milk from a Hamilton dairy goat farm was heated to $45^{\circ} \mathrm{C}$ and centrifuged at $1650 \mathrm{~g}$ for 30 minutes and then had the fat removed. The milk was reheated to $45^{\circ} \mathrm{C}$, the $\mathrm{pH}$ was adjusted to 4.2 using $2 \mathrm{M} \mathrm{HCl}$, stirred for 30 minutes and centrifuged at $1650 \mathrm{~g}$ for 15 minutes to recover the precipitated caseins. These were resuspended four times in 10 times the original milk volume and stirred for 60 minutes before recentrifugation at $1650 \mathrm{~g}$ for 15 minutes. After the washes, the casein was freeze-dried, vacuum packed and stored at $4{ }^{\circ} \mathrm{C}$ until further use. Upon use, the caseins were dissolved in Dulbecco's phosphate buffered saline (PBS; Invitrogen, Auckland, New Zealand) at $37^{\circ} \mathrm{C}$ for 60 minutes and then sonicated to further solubilise them. The final LPS concentrations were in the range 150-300 EU/mg for each. $\alpha$ s1 casein was purified from milk from the same source by high pressure liquid chromatography. Bovine $\beta$-lactoglobulin and caprine $\beta$-lactoglobulin were also provided by Ali Hodgkinson, and were purified by Trichloroacetic acid (TCA) precipitation. 


\subsubsection{Mice}

Mice were aged 6-12 weeks at the start of experiments. BALB/c mice were from two sources. Early experiments in BALB/c mice used mice originally sourced from the Wellington School of Medicine maintained at the Biomedical Research Unit (BRU). The BALB/c breeding colony was replaced with BALB/c-ByJ mice sourced from The Jackson Laboratory (Bar Harbor, ME) in 2011 and these were used in later experiments. KN2 mice[373] were donated by Richard Locksley (University of California at San Francisco, CA). G4 mice[273] were donated by William Paul (National Institutes of Health, Bethesda, MD). IL-3-/- mice have been described[309, 395, 396] and were donated by Booki Min (The Cleveland Clinic Foundation Biological Resources Unit, Cleveland, OH). Mice with an IL-9 transgene under the control of the intestinal rat fatty acid binding protein promoter (iFABPp-IL-9)[57] were a gift from Klaus Matthaei (John Curtin School of Medical Research, Australian National University, Canberra, Australia). All strains were backcrossed to a BALB/c background prior to arrival at the BRU, except $i F A B P p$-IL-9 mice which were generated using BALB/c blastocysts[57]. KN2/KN2 and G4/G4 homozygotes were crossed to BALB/c-ByJ mice to generate $\mathrm{KN} 2 /+$ and $\mathrm{G} 4 /+$ mice, respectively. $\mathrm{KN} 2 / \mathrm{G} 4$ mice were the $\mathrm{F} 1$ progeny of a KN2/KN2 by G4/G4 cross. To generate $i F A B P p$-IL-9-IL-3-/- mice, IL3-/- mice were first crossed with an $i F A B P p$-IL-9 homozygote. F1 mice were crossed to IL-3-/- and F2 pups that had no detectable IL-3 gene and carried the transgene were used as $i F A B P p$-IL-9-IL-3-/- parental mice. These F2 mice interbred with IL3-/- mice were used as the source of $i F A B P p$-IL-9-IL-3-/- and IL-3-/- sib-matched controls in the F3. P1 iFABPp-IL-9 mice were used as $i F A B P p$-IL-9-IL-3+/+ mice in these experiments. Experimental mice were maintained in a 14/10 hour light-dark cycle at the BRU at the Malaghan Institute of Medical Research, Victoria University of Wellington. Mice were euthanised by either cervical dislocation or carbon dioxide asphyxiation. Mice were used in accordance with the permissions of the Victoria University of Wellington Animal Ethics Committee. 


\subsubsection{Genotyping}

Tail tips or ear clips of mice were lysed in $75 \mu \mathrm{L}$ lysis buffer $(25 \mathrm{mM}$ sodium hydroxide, $0.2 \mathrm{mM}$ Ethylenediaminetetraacetic acid (EDTA; Invitrogen) in deionised $\mathrm{H}_{2} \mathrm{O}$ for $60-120$ minutes at $95^{\circ} \mathrm{C}$. Lysis was stopped by addition of $75 \mu \mathrm{L} 40 \mathrm{mM}$ Tris-HCl. Debris was removed with a quickspin in a benchtop centrifuge and genomic (g)DNA was quantified by nanodrop (A260/280) and stored at $4{ }^{\circ} \mathrm{C}$ until being run through the polymerase chain reaction (PCR). Primers against wild-type Il9 and the $i F A B P p$-IL-9 transgene were purchased from Sigma and were as follows: iFABP (forward) construct: GTGGACAGGACCGAATCTCTGC. Il9 gene (forward): CAGGTTTGAGGGAAGCTGGTCC; 3' IL9 reverse primer: GCCTTTGCATCTCTGTCTTCTGG. PCR amplification was performed using the iTaq ${ }^{\mathrm{TM}} \mathrm{DNA}$ polymerase pre-mix kit (BioRad, Hercules, CA, USA) . A master reaction mix consisting of 1x Magnesium-free master mix buffered with $2 \mathrm{mM} \mathrm{MgCl}_{2}, 400 \mathrm{nM}$ iFABP (forward), $70 \mathrm{nM}$ Il9 gene (forward), $400 \mathrm{nM}$ Il9 reverse primer, $200 \mu \mathrm{M}$ dNTPs, 0.2 $\mu \mathrm{L}$ iTaq ${ }^{\mathrm{TM}}$ DNA polymerase to which $300 \mathrm{ng}$ template DNA were added to a total volume of $25 \mu \mathrm{L}$. Primer aliquots were stored as $100 \mu \mathrm{M}$ stocks and pre-diluted such that $0.5 \mu \mathrm{L}$ would give the appropriate concentration $(20 \mu \mathrm{M}, 3.5 \mu \mathrm{M}$ and $20 \mu \mathrm{M}$ respectively). The PCR cycle was as follows: Stage 1: $94^{\circ} \mathrm{C} 3$ minutes; Stage 2: 40x $94{ }^{\circ} \mathrm{C} 20 \mathrm{~s} 64^{\circ} \mathrm{C} 20 \mathrm{~s}, 72^{\circ} \mathrm{C} 20 \mathrm{~s}$; Stage $3: 72^{\circ} \mathrm{C} 3$ minutes followed by an infinite hold at $4{ }^{\circ} \mathrm{C}$. Amplified products were run on a gel made from 1-1.5\% agarose (Invitrogen) and the presence of the $i F A B P p$-IL-9 transgene was detected as an approximately 400 bp product. PCR success was evaluated by the presence of a 200 bp WT band.

The same lysis conditions were used for the $I l 3$ gene. Primers (Sigma) against the gene encoding murine IL-3 amplified a were as follows: Forward primer: 5'GAACCTGAACTCAAAACTGAT3' Reverse primer: 5'CTGAAGATTGGACTTGATAAC.

A 1x master mix was made to give a final reaction volume of $20 \mu \mathrm{L}$ containing $500 \mathrm{nM}$ of the forward and reverse primer and $0.2 \mu \mathrm{L} \mathrm{iTaq}^{\mathrm{TM}} .200-500 \mathrm{ng}$ DNA were added to each tube and the amplification cycle was as follows: Stage 1: $94{ }^{\circ} \mathrm{C} 2$ minutes; Stage 2: $94.0^{\circ} \mathrm{C} 20 \mathrm{~s}, 56.1^{\circ} \mathrm{C} 10 \mathrm{~s}, 72.0^{\circ} \mathrm{C} 30 \mathrm{~s}$; stage $3: 72^{\circ} \mathrm{C} 5$ minutes followed by an infinite hold at $4{ }^{\circ} \mathrm{C}$. The reaction was predicted to yield an $1131 \mathrm{bp}$ fragment and IL3-/- mice were identified as those in which the wild-type IL-3 band was not detected. 
The success of the PCR in IL-3-/- mice was further confirmed by the presence of the wild-type IL-9 gene. In the F2, IL-3+/- and IL-3-/- mice were born in approximate Mendelian ratios.

\subsubsection{Protein quantification}

Protein was quantified by nanodrop or using the Bicinchoninic acid $\mathrm{BCA}^{\mathrm{TM}}$ protein assay kit (Thermoscientific, Rockford, IL, USA) according to the manufacturers recommendations with the following modifications. Samples, standards and reagents were added in $30 \mu \mathrm{L}$ volumes. After 30 minutes incubation at $37^{\circ} \mathrm{C}$ plates were removed from the incubator and read at $562 \mathrm{~nm}$ on a Versamax plate reader.

\subsubsection{Trypan blue exclusion test}

Samples were diluted 1:1 with 0.4\% trypan blue (Invitrogen). Between 30 and 200 cells were counted and these were back-calculated to the total volume for numbers.

\subsubsection{Prediction of sequence homology using DELTA BLAST}

The sequence for cow $\beta$-lactoglobulin was taken from Alexander et al.[397]. A proteinprotein DELTA-BLAST search[398] was performed using the http : //blast.ncbi.nlm.nih.gov/ search engine and search species were Homo sapiens (taxid:9606), Mus musculus (taxid:10090) and Bos taurus (taxid:9913). Similar homologies to those reported by Restani et al. [169] were observed for $\alpha \mathrm{s} 1$ and $\alpha_{\mathrm{s}} 2$ caseins (not shown). The query sequence for BLG was ALTCGAQALIVTQTMKGLDIQKVAGTWYSLAMAASDISLLDAQSAPLRVY VEELKPTPEGDLEILLQKWENDECAQKKIIAEKTKIPAVFKIDALNENKVLV LDTDYKKYLLVCMENSAEPEQSLVCQCLVRTPEVDDEALEKFDKALKALPMHIRLSFNPTQLEEQCHI. The glycodelin[244] and human placental precursor protein-14[245] proteins, both of which have been previously identified as $\beta$ lactoglobulin-related proteins[244, 245], each returned 44\% homology. 


\subsubsection{Statistical analysis and graphics}

Data were graphed and analysed in Graphpad Prism 5.0. Statistical analyses used are stated in figure legends and $\mathrm{p}<0.05$ was considered as significant. When multiple individual group comparisons yielded similar significance values (i.e., were commonly $<0.05,<0.01$ etc.), the significance value is indicated for all connected lines. In comparisons between two similarly-sized groups, Student's $t$-test was used. Analyses examining a single parameter of three or more groups were compared with One-way ANOVA with Tukey's post-test. For experiments examining two or more groups, Twoway ANOVA with Bonferroni's post-test were used. Repeated measures tests were used in cases in which the same biological sample was compared. Figures showing data combined from multiple experiments were first normalised such that baseline values were similar across combined experiments. Unless otherwise stated, symbols represent individual mice and error bars represent mean \pm the standard error of the mean (SEM).

pTh2 assays were designed to detect 2 -3-fold differences with $80 \%$ power $(1-\beta ; \beta=0.2)$ at $\alpha=0.05$ in experiments using one or more experiments including groups of $5-10$ mice. The calculation employed was:

$\mathrm{n}=(\mathrm{Z} 2 \alpha+\mathrm{Z} \beta) \mathrm{x}(\mathrm{Z} 2 \alpha+\mathrm{Z} \beta) \mathrm{x}((\mathrm{SD} 1) \mathrm{x}(\mathrm{SD} 1)+(\operatorname{sd} 2) \mathrm{x}(\operatorname{sd} 2)) /(\mathrm{ED})$

Where, ' $\mathrm{n}$ ' is the number of mice required per group, 'ED' is the expected difference $\left(\mu_{1}-\mu_{2}\right)$, 'SD1' is the empirical SD for the responding immunogen, and 'sd2' is the sd of the group for comparison. For experimental groups, 'sd2' was approximated as half the expected $\mu_{2}$. Empirical values attained using mice $(\mathrm{n}=298)$ immunised intradermally with 600 dead $i$ L3 $N$. brasiliensis larvae give $\mu=226379 \pm 133931$ Th2 cells; ( $\mathrm{n}=27$ ) HDM-immunised G4/ nodes $\mu=87021 \pm 39275$; control lymph nodes $(\mathrm{n}=43) \mu=2018 \pm 1444$. To fit with historical data and reduce the occurrence of type-1 errors, pTh2 data were not log-transformed prior to analysis. An example derivation for HDM power is shown:

To detect an $x$-fold difference to an allergen response on a similar scale to HDM immunization in 80/100 experiments $(\beta=0.2)$ :

$(x=1.2, \mathrm{n}=71.8)$; 
$(x=2, \mathrm{n}=8.4)$;

$(x=3, \mathrm{n}=4.1)$;

$(x=4, \mathrm{n}=3.1)$;

$(x=5, \mathrm{n}=2.6)$;

$(x=10, \mathrm{n}=2.0)$ mice/group.

Figures were prepared in AdobeßIllustrator@version 5.1.

\subsection{Cell culture}

During preparation, cells were handled in Iscove's modified Dulbecco's medium + Glutamax (IMDM; Invitrogen). Base culture medium consisted of $5 \%$ or $10 \%$ foetal calf serum (Sigma), $100 \mathrm{U} / \mathrm{mL}$ Penicillin/100 $\mu \mathrm{g} / \mathrm{mL}$ Streptomycin (Invitrogen), $55 \mu \mathrm{M}$ $\beta$-mercaptoethanol (Invitrogen), in IMDM (cIMDM). Standard incubation conditions of $37^{\circ} \mathrm{C}$ humidified in $5 \% \mathrm{CO}_{2}$ in air were used.

\subsubsection{Hybridoma culture}

GK1.5 (process similar for other antibodies made in house) anti-CD4 monoclonal antibody was prepared in house from the culturesupernatant of the GK1.5 hybridoma[399]. The GK1.5 hybridoma was grown by culture in 10\% cIMDM and antibody was isolated from the supernatant by fast pressure liquid chromatography using Hitrap protein G affinity columns (GE Healthcare) used according to the manufacturer's instructions. After isolation into neutralising buffer, antibody was dialysed for 2-4 hours in 2L PBS using 10000 molecular weight cut-off (MWCO) cassettes (Pierce). The PBS was changed and antibody was dialysed overnight. The next day, dialysed protein was extracted from the cassette and concentration was determined by nanodrop absorption at $280 \mathrm{~nm}$. Antibody was concentrated to $2.5 \mathrm{mg} / \mathrm{mL}$ in $10000 \mathrm{MWCO}$ or 30000 MWCO vivaspin microtubes (GE Healthcare). Heavy and light chain expression was confirmed by sodium dodecyl sulfate gel electrophoresis, and in some cases, in vivo depleting capacity was assessed. Mice received $0.5 \mathrm{mg}(200 \mu \mathrm{L})$ antibody i.p. with each injection according to standard protocols[400], and in maintenance experiments, antibody was readministered every four days. On days of challenge plus monitoring 
for peripheral CD4+ T cell content, monitoring was done prior to gavage and prior to GK1.5 administration. Mock-depleted mice received $0.5 \mathrm{mg}$ polyclonal Rat IgG purified from naive rat sera purified in house by Hitrap protein $G$ affinity or was purchased from Sigma.

\subsubsection{Bone marrow cell culture for IL-3- and TSLP-elicited basophils}

Bone marrow cells were isolated as described in mouse manipulations. Bone marrow cells were seeded at $1 \times 10^{6} / \mathrm{mL}$ in 24 -well plates. Cells were cultured in the presence of $10 \mathrm{ng} / \mathrm{mL}$ IL-3 (Peprotech, Rocky Hill, NJ, USA), $1 \mu \mathrm{g} / \mathrm{mL}$ TSLP (R\&D Systems, Minneapolis, MN, USA), $10 \mathrm{ng} / \mathrm{mL}$ IL-3 plus $1 \mu \mathrm{g} / \mathrm{mL}$ TSLP, or an equivalent volume of $1 \% \mathrm{BSA} / \mathrm{PBS}$ as medium alone controls. After three days, half of the medium was removed without disrupting the cells and replaced with fresh medium containing $10 \mathrm{ng}$ IL-3, $1 \mu \mathrm{g}$ TSLP, $10 \mathrm{ng}$ IL-3 plus $1 \mu \mathrm{g}$ TSLP or an equivalent amount of

$1 \% \mathrm{BSA} / \mathrm{PBS}$ as seeded. On day five, non-adherent cells were harvested, counted by trypan blue exclusion, and analysed by flow cytometry. By day five, adherent cells in IL-3-containing cultures had reached confluence and the medium had changed colour. TSLP containing cultures were predominantly non-adherent, however, some sparse adherent cells were observed extending pseudopods; these were also observed occassionally in medium-only cultures. FceRI+cKit- cells comprised approximately $0.8 \%$ of bone marrow cells on day 0 , and at seeding of G4/ + cultures, $60 \%$ of these cells were $\mathrm{GFP}+$.

\subsubsection{Th cell polarization}

Twenty-four-well plates (BD) were coated overnight at $4{ }^{\circ} \mathrm{C}$, or for 2 hours at $37^{\circ} \mathrm{C}$ with anti-CD3 in PBS (2C11, $1 \mu \mathrm{g} / \mathrm{mL}, 300 \mu \mathrm{L} /$ well; in house). On the day of the assay, plates were washed twice with $500 \mu \mathrm{L}$ IMDM and $1 \times 10^{6}$ splenocytes from naive mice of the specified genotype were added to each well in a $1 \mathrm{~mL}$ culture volume. Cultures were left untouched for three days, at which point wells were resuspended and half of the cells were removed and discarded and $500 \mu \mathrm{L}$ of $2 \mathrm{x}$ conditioning medium was 
again added to wells. On day 4, $500 \mu \mathrm{L}$ was removed without resuspension and cells were fed as on day 3. For restimulation assays, cells were removed from wells on day 5 , washed twice in IMDM and replated in wells containing $100 \mathrm{U} / \mathrm{mL}$ IL-2 and anti-IL-4 (11B11, $10 \mu \mathrm{g} / \mathrm{mL}$; prepared in house) as the final culture conditions. During the rest period, half of the medium was replaced every day and if the medium reached pH7.0 or less in any culture, determined by a change in phenol red colouring, the contents of the wells were split in two. Separate wells were maintained for individual mice from the start of culture throughout the process, such that data points represent biological replicates unless explicitly stated. For initial conditioning, Th0 medium contained 100 $\mathrm{U} / \mathrm{mL}$ IL-2, $10 \mu \mathrm{g} / \mathrm{mL}$ anti-IL-4 and $10 \mu \mathrm{g} / \mathrm{mL}$ anti-IFN- $\gamma$ (AN.18; prepared in house). Th1 medium contained $100 \mathrm{U} / \mathrm{mL}$ recombinant (r)human IL-2, $10 \mathrm{ng} / \mathrm{mL}$ rIL-12p40 and $10 \mu \mathrm{g} / \mathrm{mL}$ anti-IL-4. Th2 medium contained $100 \mathrm{U} / \mathrm{mL}$ IL-2 and $1000 \mathrm{U} / \mathrm{mL}$ chinese hamseter ovary (CHO)-mIL-4 (IL-4 activity was compared by ELISA using standards originally calibrated using the CT.4S cell line as described[342]. One unit is equivalent to approximately $0.5 \mathrm{pg}$ IL-4/mL[342]). During primary stimulation, all cultures contained $20 \mu \mathrm{L} / \mathrm{mL}$ anti-CD28 (37.51; grown and titrated in house without purification) that was not replaced during subsequent feeds.

\subsubsection{In vitro restimulation of Th cells}

After conditioning, Th cells were transferred to wells coated with anti-CD3 at 10 $\mu \mathrm{g} / \mathrm{mL}$ as per vanPanhuys et al.[1]. Restimulation medium included $100 \mathrm{U} / \mathrm{mL}$ IL-2 and $20 \mu \mathrm{L} / \mathrm{mL}$ anti-CD28. In experiments examining IL-4 protein production, antiIL-4 was also included in the restimulation medium. For the detection of intracellular cytokines, $4 \mu \mathrm{L}$ Golgistop ${ }^{\mathrm{TM}}$ containing monensin (BD) was combined with $16 \mu \mathrm{L}$ IMDM and $20 \mu \mathrm{L}$ was added to each well two hours before the cells were harvested. In experiments that simultaneously examined cytokine production, parallel cultures to which monensin was not added were used. 


\subsubsection{IgG1 spot assay}

MAIPSWU10 $0.45 \mu \mathrm{m}$ hydrophobic Immobilon P memprane 96-well ELISpot filter plates (Millipore, Billerica, MA) were pre-soaked for 90 seconds with $50 \mu \mathrm{L} 70 \%$ ethanol. After removing the ethanol, plates were washed five times with $200 \mu \mathrm{L}$ PBS and incubated overnight at $4{ }^{\circ} \mathrm{C}$ with $10 \mu \mathrm{g} / \mathrm{mL}$ of the coating antigen. The following day plates were washed five times with $200 \mu \mathrm{L}$ PBS and were left at room temperature with 10\% cIMDM to block unbound sites on the plate. After processing, spleen and/or bone marrow cells were resuspended in 10\% cIMDM, and were serially diluted and transferred to spot assay plates. Plates were given a soft spin (50 g for $60 \mathrm{~s}$ ) and overlaid with a further $50 \mu \mathrm{L}$ assay medium and incubated for 4 hours at $37^{\circ} \mathrm{C}$. After incubation, plates were washed five times with $200 \mu \mathrm{L}$ PBS and then incubated with $0.91 \mu \mathrm{g} / \mathrm{mL}$ biotinylated rat anti-mouse IgG1 (A85-1; BD) overnight at $4{ }^{\circ} \mathrm{C}$. Plates were washed a further four times with PBS and incubated with $1 / 1000$ SPX for 1 hour at room temperature in the dark and detected with the 3-amino-9ethylcarbazole AEC Chromogen staining kit (Sigma) resuspended in deionized water according to the manufacturers instructions and $40 \mu \mathrm{L}$ was added to each well. Fortyfive minutes later, plates were washed with tap water and put at $37^{\circ} \mathrm{C}$ in air until dry. Spots formed a red precipitate and were enumerated by eye and validated using images acquired on an ELIspot reader. Concentrations at which densities were to numerous to count were not included in quantitations; otherwise all dilutions were included in frequency calculations.

\subsection{Mouse manipulations}

\subsubsection{Primary Th2 (pTh2) assay}

After anaesthesia with $200 \mu \mathrm{L}$ anaesthetic solution $(100 \mathrm{mg} / \mathrm{Kg}$ ketamine, $3 \mathrm{mg} / \mathrm{Kg}$ Xylazine), mice were intradermally injected in the outer ear pinna (generally the left ear) with $30 \mu \mathrm{L}$ of immunogen in PBS. The draining auricular lymph node was later excised and examined for live cells by trypan blue exclusion and for proportional GFP expression in CD4+ T cells by flow cytometry. Generally, analyses were performed 
seven days after innoculation. When data were compiled from multiple experiments the proportion of GFP + cells or hCD2+ cells were normalised to $0.04<\mathrm{x}<0.05 \%$ of CD4+ T cells in wild-type $(+/+)$ mice immunized with either dead $i$ L3 N. brasiliensis larvae or HDM. In cow and goat milk comparisons, casein samples had low solubility in PBS, therefore three separate injections were required and administered in the centre of the pinna, then on then in two additional injections perpendicular to the centre of the pinna. Controls also received $3 \times 30 \mu \mathrm{L}$ injections in these experiments. In pTh2 assays that also examined B cell responses, the total number of IgG1+IgD-B220+ cells was determined by multiplying the proportion of live cells that were IgG1+IgDB220+, as determined by flow cytometry, by the total number of live cells found in lymph nodes.

\subsubsection{Oral allergen-induced experimental anaphylaxis (diar- rhoea) model}

A model of oral allergen induced diarrhoea was employed as established[37]. Mice were sensitized intraperitoneally (ip) twice, two weeks apart with $50 \mu \mathrm{g}$ ovalbumin (OVA; Sigma) + 1 mg Alu-gel-S (Serva Electrophoresis GmbH, Heidelberg, Germany) in $200 \mu \mathrm{L}$ using a $1 \mathrm{~mL}$ tuberculin syringe (BD) and 25-gauge needle (BD). Thriceprimed mice received an additional ip injection on day minus 14. From day 28, mice were fasted for four hours prior to gavage and subsequently challenged intragastrically three times a week with $50 \mathrm{mg}$ OVA $(200 \mathrm{mg} / \mathrm{mL})$ in PBS. Mice were monitored for diarrhoea development 60 minutes later. For serum analyses, mice were bled two hours after challenge. Mice were bled by the submandibular route using a $4 \mathrm{~mm}$ lancet (Ables scientific, Welshpool, Western Australia) or were pre-heated under a heat lamp followed by lateral tail vein bleeding using a \#10 carbon steel surgical blade (Swann-Morton, Sheffield, England). When applicable, CD4+ T cells were depleted with ip injections of $0.5 \mathrm{mg}$ GK1.5 on days 25, 26 and 27, or the three days following challenge ten. Mock-depleted mice received polyclonal IgG purified from Hooded Lister rat serum. For long-term depletions, booster injections were administered every four days. 


\subsubsection{Active systemic anaphylaxis}

Mice were warmed with a heatlamp for 3 minutes and then received $100 \mu \mathrm{g}$ OVA in PBS intravenously through a 29-gauge needle (BD). Intravenous challenges were done two or three days after mice received their final gavage challenge. Temperatures were monitored using a RET-3 rectal temperature probe attached to a digital thermometer (Coherent Scientific, Hilton, South Australia).

\subsubsection{Packed Cell Volume (PCV)}

Haematocrit was taken directly from a tail nick and was most pronounced 45-60 minutes after challenge. $75 \mathrm{~mm}$ heparinized capillary tubes (Drummond scientific, Broomall, PA) were used to take haematocrit and were plugged with plasticine. Tubes were spun at $8000 \mathrm{rpm}$ for 5 minutes on a benchtop centrifuge. PCV was assessed using a sliding scale and represents proportion of the total volume that were red blood cells.

\subsubsection{Serum isolation}

Blood was left to clot overnight at $4{ }^{\circ} \mathrm{C}$. Blood was spun for 3 minutes at 4 degrees $\left(\mathrm{RCF}_{\max }=1523\right)$, serum removed and frozen at $-20^{\circ} \mathrm{C}$ until analysed by ELISA.

\subsubsection{Tissue isolation}

After euthanasia, mice were rinsed with $70 \%$ ethanol. Depending on the requirements, the spleen, mesenteric and auricular lymph nodes were isolated by blunt dissection with forceps. Bone marrow was removed as outlined below. Colons and caecae were excised afterwards and the jejunal tissue was removed by cutting away the mesenteries. The first $1.5 \mathrm{~cm}$ of the intestine proximal to the stomach was deemed the duodenum. The next 1-1.5 cm of jejunal tissue was used for histological sections, and for cell isolation the next $5 \mathrm{~cm}$ section of jejunum was used. Jejunal tissue for 
cell analyses were flushed with $5 \mathrm{~mL} \mathrm{Ca}{ }^{2+}$ and $\mathrm{Mg}^{2+}$-free Hanks' Balanced Salt Solution (HBSS; Invitrogen) using an 18-gauge needle (BD) and were stored in $\mathrm{Ca}^{2+}$ and $\mathrm{Mg}^{2+}$ free HBSS on ice. All other tissues were stored in IMDM on ice until subsequent processing.

\subsubsection{Processing of jejunal tissue}

After vigorous shaking with forceps to dislodge mucus and remaining gut content, Peyer's patches were excised and put on ice. Jejunal sections were transferred to 6-well plates (BD) containing $5 \mathrm{~mL}$ stripping buffer (5 mM EDTA), $25 \mathrm{mM}$ HEPES (Sigma), $10 \%$ FCS in $\mathrm{Ca}^{2+}$ and $\mathrm{Mg}^{2+}$ free HBSS) pre-heated to $37^{\circ} \mathrm{C}$. Tissues were incubated in air at $37^{\circ} \mathrm{C}$ with shaking $(100 \mathrm{rpm})$ for five minutes. Tissues were vigorously shaken with forceps to remove stripped epithelial cells. The process was repeated two more times for a total of 15 minutes stripping. Tissues were rinsed in 5 $\mathrm{mL} 4{ }^{\circ} \mathrm{C} \mathrm{Ca}^{2+}$ and $\mathrm{Mg}^{2+}$ free HBSS and placed on tissue paper to remove moisture. Jejunal tissue was chopped into pieces small enough to pass through a $1 \mathrm{~mL}$ pipette tip and incubated with shaking for 30 minutes in $5 \mathrm{~mL} 2.4 \mathrm{mg} / \mathrm{mL}$ collagenase A from Clostridium histolyticum (Roche Diagnostics Gmbh, Mannheim, Germany) in IMDM. The solution was subsequently passed through a 21-gauge needle (BD) with pressure on the bottom of the plate and incubated for a further 15 minutes at $37^{\circ} \mathrm{C}$ with shaking. Debris was removed by passing through two layers of cotton gauze (Sefar Filter Specialists Ltd., Te Papapa, Auckland, NZ) and rinsed with $10 \mathrm{~mL}$ cIMDM or flow buffer and then through a $70 \mu \mathrm{m}$ cell strainer (BD). Cells were then processed as per standard flow cytometric procedures, but all isolated cells were stained for flow cytometry.

\subsubsection{Bone marrow preparation}

The left femur was used for bone marrow isolation. The leg was washed with $70 \%$ ethanol and the surrounding muscle was removed. The tibia was disconnected by dislocating the knee and then using forceps, the bone was removed by cutting under the hip socket. Bones were cleaned and stored in IMDM on ice until processing. To 
process bones, both ends were cut and a 22-gauge needle (BD) was inserted into the bone tip. Five mL IMDM was flushed through the bone to isolate marrow, which was spun down and resuspended in red blood cell lysis buffer (Sigma) for 2 minutes at room temperature. Lysis was stopped by addition of $13 \mathrm{~mL} \mathrm{5 \%} \mathrm{cIMDM.} \mathrm{Cells} \mathrm{were}$ again spun and then resuspended in 10\% cIMDM for the spot assay, 5\% cIMDM for mast cell culture, and were subsequently transferred to flow buffer ( $2 \%$ FCS, $0.01 \%$ w/v Sodium Azide (Sigma) in dPBS) for staining.

\subsubsection{Splenocytes}

After excision from the mouse, spleens were placed on a $70 \mu \mathrm{m}$ cell strainer and dissociated with pressure against the strainer using the rubber end of a $1 \mathrm{~mL}$ tuberculin syringe (BD) and rinsed with IMDM. Cells were spun down and resuspended in 2 $\mathrm{mL}$ red blood cell lysis buffer for 2 minutes at room temperature. The reaction was quenched with $13 \mathrm{~mL} 5 \%$ cIMDM or flow buffer and then tubes were spun down for 4 minutes at $1400 \mathrm{rpm}(419 \mathrm{x} \mathrm{g})$. Spleen cells were then resuspended in $10 \mathrm{~mL}$ buffer for counting. Cells were respun and resuspended for future use.

\subsubsection{Lymph node cells}

Mesenteric, auricular lymph nodes and Peyer's patch cells were liberated by crushing lymph nodes through a $70 \mu \mathrm{m}$ cell strainer with the rubber end of a $1 \mathrm{~mL}$ tuberculin syringe (BD) and rinsed with flow buffer for flow cytometry. Cells were spun and resuspended in $4 \mathrm{~mL}$ buffer for counting. Red blood cells were ignored during counting and were identified as small, dense, spherical cells with a darker hue than lymphocytes. For flow staining, cells were transferred through $70 \mu \mathrm{m}$ gauze and stained for flow cytometry.

\subsubsection{Jejunal mast cell quantification}

Jejunal tissue sections were removed from mice and put in $10 \%$ formalin $(4 \% \mathrm{w} / \mathrm{v}$; Sigma) and stored at $4{ }^{\circ} \mathrm{C}$ until embedded in paraffin. Tissues were processed into 4 
$\mu \mathrm{m}$ sections using standard histological techniques by Jane Anderson of the Wellington School of Medicine. Slides were stained with chloroacetate esterase (CAE) stain and lightly counterstained with hematoxylin as described[37, 57]. Mast cells were identified as CAE positive cells, which were easily identified as bright red cells as previously described[37, 57]. Four randomly selected high power fields $(550 \mu \mathrm{m} /$ field; $400 \mathrm{x}$ magnification using $0.22 \mathrm{~mm}$ Field Number 10x ocular and 40x objective lens) were examined from each mouse.

\subsection{Flow cytometry}

\subsubsection{Flow Cytometry reagents}

A full list of the flow cytometry reagents is included in the appendices. The following viability dyes were used: 4,6-diaminidino-2-phenylindole (DAPI; Molecular Probes); 7-Amino Actinomycin D (7-AAD; BD Pharmingen). For intracellular staining, Live/Dead@ fixable blue (Invitrogen) was used in place of DAPI.

Antibodies against the following molecules, and secondary reagents were purchased from BD Pharmingen, unless otherwise specified (clone): B220-V450 (RA3-6B2); B220-PE-Texas Red@(Ra3-6B2); CD3-AF488 (500A2; Invitrogen Molecular Probes); CD4-APC (RM4.5); CD4-APC-H7 (GK1.5); CD4-FITC (GK1.5; Prepared in house); CD4-PE (GK1.5); CD4-Percp (RM4.5); CD4-Qdot605@ (RM4.5; Invitrogen Molecular Probes); CD8a-AF700(536.7; Biolegend); CD8a-PE/Cy7 (53-6.7); CD19-APCH7 (ID3); CD44-PE (IM7); CD45-Pacific blue ${ }^{\mathrm{TM}}$ (30F11; Biolegend); CD62L-Biotin (MEL-14); CD62L-Pacific blue (MEL-14; Biolegend); CD117(cKit)-APC (2B8); FceRIPE (Mar-1; eBioscience); FcgRII/III-FITC (2.4G2); human CD2-APC (RPA2.10); Ia/Ie-PE/Cy7 (M51114.15.2; Biolegend); IL-3-PE (MP2-8F8); IL-4-APC (11B11); IL4-PE (11B11); IL-5-PE (TRFK5); IL-10-PE (JES5-16E3); IL-13-PE (eBio13A; eBioscience); IgD-PE (11-26c.2a); IgE-biotin (R35-118); IgG1-biotin (A85-1); Pan NKPE/Cy7 (DX5; eBioscience); Streptavidin-APC; Streptavidin-FITC; Streptavidin-PE; Streptavidin-PE-TexasRed@. 


\subsubsection{Surface staining}

After tryan blue exclusion, $1.25 \times 10^{6}$ cells were transferred to $5 \mathrm{~mL}$ flow-compatible tubes through $70 \mu \mathrm{m}$ gauze. Flow buffer containing $50 \mu \mathrm{L}$ anti-CD16/32 antibody (24G2) made in house was added to each tube for five minutes which was then incubated with $50 \mu \mathrm{L}$ containing the primary antibody mix. Samples were incubated for 30 minutes at $4{ }^{\circ} \mathrm{C}$ and then washed with $4 \mathrm{~mL}$ flow buffer $(2 \%$ FCS, $0.01 \%$ sodium azide in PBS) and resuspended for flow. For secondary antibody incubation, cells were instead resuspended in $100 \mu \mathrm{L}$ containing optimally diluted secondary reagent.

\subsubsection{Intracellular staining}

Cells were harvested and transferred to flow-compatible tubes containing $3 \mathrm{~mL} 4{ }^{\circ} \mathrm{C}$ PBS. Tubes were spun and resuspended in $1 \mathrm{~mL}$ PBS for counting. Cells were respun and incubated with $1 \mathrm{~mL} \mathrm{1/1000} \mathrm{Live/dead} \mathrm{fixable} \mathrm{blue} \mathrm{in} \mathrm{PBS} \mathrm{for} 30$ minutes at $4{ }^{\circ} \mathrm{C}$ in the dark. Cells were washed with $3 \mathrm{~mL}$ flow buffer and were subjected to normal surface staining methods. After staining, cells were fixed and permeabilized using the Fix \& Perm® cell permeabilization kit (Invitrogen) according to the manufacturers instructions with the following modifications. Cells were fixed for 15 minutes using 'Reagent A' at room temperature, followed by quenching with $4 \mathrm{~mL}$ room temperature flow buffer. Samples were split in two at this stage and spun down at $1400 \mathrm{rpm}$ for 4 minutes at room temperature. Intracellular stains for analysis and in some cases, isotype controls, were added diluted to $100 \mu \mathrm{L}$ volumes of reagent B. After spinning down the cells, Intracellular stains or isotypes were added to samples and incubated for 20 minutes at room temperature. The staining was stopped by addition of flow buffer and samples were spun down then resuspended for flow cytometry.

\subsubsection{Flow cytometers}

A table of cytometers, lasers and filters used to detect specific fluorophores is included in the appendices. 
Samples were analysed on an LSR-II special order product (BD) equipped with the following lasers. Fluorophores were detected as specified. Numbers indicate filter and the letter denotes the position of the detector in the flow cube. Ultraviolet laser (355 nm): DAPI 450/50B, Live/Dead fixable blue 450/50B. Violet laser (405

nm): Brilliant Violet 421 450/50F, Pacific Blue 450/50F, Horizon V450 450/50F, Qdot-605 605/40C. Blue laser (488 nm): PerCp 705/70A, GFP B515/20B, FITC B515/20B, Alexafluor488 B515/20B, Side Scatter 488/10C. Green laser (532 nm): PECy7 780/60A, 7-AAD 660/40C, PE-Texas Red@ 610/20D, PE G575/26E. Red laser (633/640 nm): APC-Cy7 780/60A, APC-H7 780/60A, Alexafluor-700 710/50B, APC $670 / 14 \mathrm{C}$. In some experiments a BD FACSCalibur was used. For these experiments 7 -AAD was used as the live/dead stain in place of DAPI. The configuration for these experiments was as follows: Blue laser (488 nm): One of FITC, Alexafluor-488 or GFP on the 530/30 FL1; PE 585/42 FL2; 7-AAD 670LP FL3; APC 661/16 FL4. Sorting was done on a FACSDiva equipped witha Blue (488 nm) laser with a 530/30 FL1 filter; 585/42 FL2 filter and an FL3 670LP filter. Red laser (633 nm): 661/16 FL4, 780/60 FL5. UV laser (355 nm) FL6 450/50, FL8 620LP. Sorting used DAPI in FL6 PE from FL2, and APC in FL4.

\subsection{ELISAs}

\subsubsection{Antigen-specific IgG1, IgG2a and polyclonal Ig ELISA}

Ninety-six well Immunosorp Maxi plates (Nunc) were coated overnight with 100 $\mu \mathrm{L} /$ well, $10 \mu \mathrm{g} / \mathrm{mL} \mathrm{HDM}$ (Greer laboratories), OVA, bovine or caprine BLG in $\mathrm{pH}=9.60 .1 \mathrm{M}$ carbonate buffer. The next day, plates were washed with $0.05 \%$ Tween20 (Sigma) in PBS $(\mathrm{pH}=7.4)$ on an automated plate washer (Skatron) and blocked for one hour with $200 \mu \mathrm{L} /$ well 10\% FCS/PBS (blocking buffer). Blocking buffer was removed and replaced with $100 \mu \mathrm{L}$ sera diluted in blocking buffer and incubated for two hours at room temperature in the dark. Wells were washed then incubated for 60 minutes with $100 \mu \mathrm{L} /$ well of $91 \mathrm{ng} / \mathrm{mL}$ biotinylated anti-mouse IgG1 (A85-1; BD) or $91 \mathrm{ng} / \mathrm{mL}$ biotinylated anti-mouse IgG2a (R19-15; BD) or $33.3 \mu \mathrm{g} / \mathrm{mL}$ biotinylated polyclonal goat anti-mouse Igs (cat \#553999; BD) diluted in blocking buffer, followed 
by a wash and incubation with streptavidin-conjugated horse radish peroxidase (GE Healthcare, Auckland, New Zealand) for 60 minutes. After a final wash step, 100 $\mu \mathrm{L}$ TMB substrate (BD Pharmingen) was added and 5-20 minutes later the reaction was stopped with $50 \mu \mathrm{L} 0.5 \mathrm{M}$ sulphuric acid. The absorbance was read at $450 \mathrm{~nm}$ on a Versamax plate reader. Serum titres were calculated in Graphpad's Prism 5.0 and were defined as the EC50 O.D. of serum dilution curves, constrained to plateau at 0.0, and a shared group maxima based on the average highest O.D. value in the series. EC50s were calculated for each mouse and their reciprocal value is shown as the titre.

\subsubsection{IgE}

For total and OVA-specific IgE, plates were coated overnight with $100 \mu \mathrm{L} /$ well 0.5 $\mu \mathrm{g} / \mathrm{mL}$ anti-mouse IgE (6HD5; in house). Plates were washed with $0.05 \%$ Tween 20 in PBS $(\mathrm{pH}=7.4)$ and then incubated with $10 \%$ foetal calf serum (FCS) in PBS. Plates were washed and samples and standards were incubated for two hours at room temperature in the dark. In some experiments plates were instead left overnight at $4{ }^{\circ} \mathrm{C}$. Plates were washed and then incubated with $100 \mu \mathrm{L} /$ well $3 \mu \mathrm{g} / \mathrm{mL}$ biotinylated Rat anti-mouse IgE (R1E4; in house; total $\operatorname{IgE}$ ) or $100 \mu \mathrm{L} 20 \mu \mathrm{g} / \mathrm{mL}$ biotinylated ovalbumin for 90 minutes at room temperature in the dark. Plates were subsequently washed and incubated with $100 \mu \mathrm{L}$ streptavidin horse radish peroxidase for 60 minutes. After a final wash step, $100 \mu \mathrm{L}$ TMB substrate was added and 10-20 minutes later the reaction was stopped with $50 \mu \mathrm{L} 0.5 \mathrm{M}$ sulphuric acid. The absorbance was read at $450 \mathrm{~nm}$ on a Versamax plate reader.

\subsubsection{Murine mast cell protease-1 (mMCP-1).}

mMCP-1 was analysed according to the manufacturers instructions. Initially kits were purchased from Moredun Scientic (Midlothian, Scotland) but became unavailable in 2011. mMCP-1 kits were subsequently purchased from eBioscience (San Diego, Ca) and again used according to the manufacturers instructions. Similar absolute values in the range of $10-40 \mathrm{ng} / \mathrm{mL}$ for naive mice and 5000-10000-fold increases in mice with 
active diarrhoea were attained using both kits.

\subsubsection{Total IgG1 and Total IgG2a}

Total IgG1 and IgG2a reagents were purchased (Bethyl Laboratories, Montgomery, Texas) and analysed according to the manufacturers instructions with some modifications to the recommended buffers. Plates were coated overnight with $100 \mu \mathrm{L}$ of a 1/100 dilution of antibody in $\mathrm{pH}=9.60 .1 \mathrm{M}$ carbonate buffer. The next day plates were washed with $0.05 \%$ Tween 20 in PBS ( $\mathrm{pH}=7.4)$ using an automated plate washer and then incubated with $10 \%$ foetal calf serum (FCS) in PBS. Plates were washed and samples and standards were incubated for two hours at room temperature in the dark. Plates were washed and then incubated with $100 \mu \mathrm{L} /$ well horse radish peroxidase-conjugated goat anti-mouse IgG1 or IgG2a diluted as recommended. Af-

ter a final wash step, $100 \mu \mathrm{L}$ TMB substrate was added and 10-20 minutes later the reaction was stopped with $50 \mu \mathrm{L} 0.5 \mathrm{M}$ sulphuric acid. The absorbance was read at $450 \mathrm{~nm}$ on a Versamax plate reader. 


\section{Chapter 3}

\section{Does the in vitro IL-4 allelic expression profile reflect functional segregation of IL-4-producing Th2 cell subsets?}

\subsection{Introduction}

\subsubsection{Monoallelic regulation of the IL-4 gene}

Studies over the past fifteen years have aimed to establish the molecular events that generate stable IL-4-producing CD4 + T cells. During these studies, the observation was made that many cells produce IL-4 from a single allele (between 52 and 85\%)[273, 374, 389]. To explain the observations, in 2005, Guo, Hu-Li and Paul argued for potential advantage of a probabilistic regulation mechanism, opperating at the single allele-level for the IL-4 gene[401]. However, these investigations have been undertaken exclusively in an in vitro context, and will benefit from elaboration in vivo.

In order to evaluate IL-4 production in vivo, IL-4 reporter systems have been developed. In our lab, we have recently combined two of these, the KN2 reporter[373] and the G4 reporter[273]. This combined reporter has the potential to record expression of each IL-4 allele individually, through the expression of two distinct reporters. In this chapter, we sought to characterise the behaviour of the KN2/G4 reporter in vitro to clarify its potential utility for in vivo investigations into the role of IL-4 in Th2 immunity. In this chapter, we used established in vitro culture methods, and IL-4 reporter systems to determine the allelic expression profile of IL-4 in vitro. We further 
test whether conditions that lead to IL-4-producing Th2 cell development in vitro also induce reporter expression in KN2/G4 cells. 


\subsection{Aims}

The experiments in this chapter were designed to evaluate the allelic expression profile of IL-4 in KN2/G4 cells in vitro.

Our specific aims were:

1) To test whether hCD2 and GFP are expressed in conditions known to promote IL-4 production;

2) To assess if IL-4 is produced from one or both alleles in KN2/G4 mice;

3) To evaluate whether IL-4 alleles are regulated independently in KN2/G4 reporter mice. 


\subsection{Results}

\subsubsection{Examining IL-4 reporter expression during primary cul- ture}

Our first experiments were designed to establish whether conditions that promote IL4 protein production also result in hCD2 and GFP expression in KN2/G4 cells. IL-4 promotes IL-4-producing Th2 cell development in vitro[342], so we began by culturing CD4+ T splenocytes on anti-CD3-coated plates in titrated doses of IL-4. Three days after seeding we used flow cytometry to examine the expression of hCD2 and GFP in KN2/G4 reporter cultures. KN2/G4 cells are IL-4-deficient, so in these experiments, the only potential source of IL-4 is exogenous.

When KN2/G4 cells were seeded in wells without any IL-4, expression of hCD2 and GFP were low (Figure 3.1A and C). CD4+ T cell numbers were similar at all concentrations of IL-4 (Figure 3.1B), but the number of cells expressing hCD2 and/or GFP increased in an IL-4 dose-dependent manner above $10 \mathrm{U} / \mathrm{mL}$ (Figure 3.1C). Therefore, IL-4 promoted reporter expression by KN2/G4 CD4+ T cells.

Our next step was to evaluate the relative expression of each allele. When we examined the allelic expression of IL-4, we observed that the majority of reporter was expressed from a single allele at all IL-4 concentrations assessed (Figure 3.2E). However, while monoallelism predominated, we also observed disparity between the absolute expression of hCD2 and GFP, because hCD2 was expressed on up to $60 \%$ of $\mathrm{CD} 4+\mathrm{T}$ cells, whereas GFP was detected in a maximum of $30 \%$ of KN2/G4 cells (Figure $3.2 \mathrm{G}$ ). While having different absolute frequencies does not preclude monoallelic regulation, to conform to an independent probabilistic regulatory mechanism, acquisition of biallelism would need to be a function of the likelihood of the expression of each individual allele. Based on this premise, independent probabilistic regulation would result in $\mathrm{P}_{\text {biallelic }}=\left(\% \mathrm{hCD} 2^{+*} \% \mathrm{GFP}^{+}\right) * 100$, where $\% \mathrm{hCD} 2^{+}$is the total percent of $\mathrm{CD} 4+\mathrm{T}$ cells expressing hCD2 (Q1 + Q2, depicted in figure 3.2C) and \%GFP ${ }^{+}$ is the total percent of CD4+ T cells expressing GFP (Q2 + Q3, depicted in figure 3.2D). By comparing the actual proportion of cells that were biallelic (Q2, depicted in figure $3.2 \mathrm{C}$ ), to the predicted proportion based on the expression of the individual 
reporters, we observed that at IL-4 concentrations above $25 \mathrm{U} / \mathrm{mL}$, biallelism was observed more frequently than would be expected if the two alleles were expressed entirely independently (Figure 3.2F). Therefore, while monoallelic expression of IL-4 predominated the response, biallelic expression was more frequent than was expected for independently-expressed alleles. Therefore, in the presence of 25 or more IL-4 $\mathrm{U} / \mathrm{mL}$, the expression of one reporter was associated with an increased likelihood of co-expressing the alternate reporter allele during primary stimulation. 


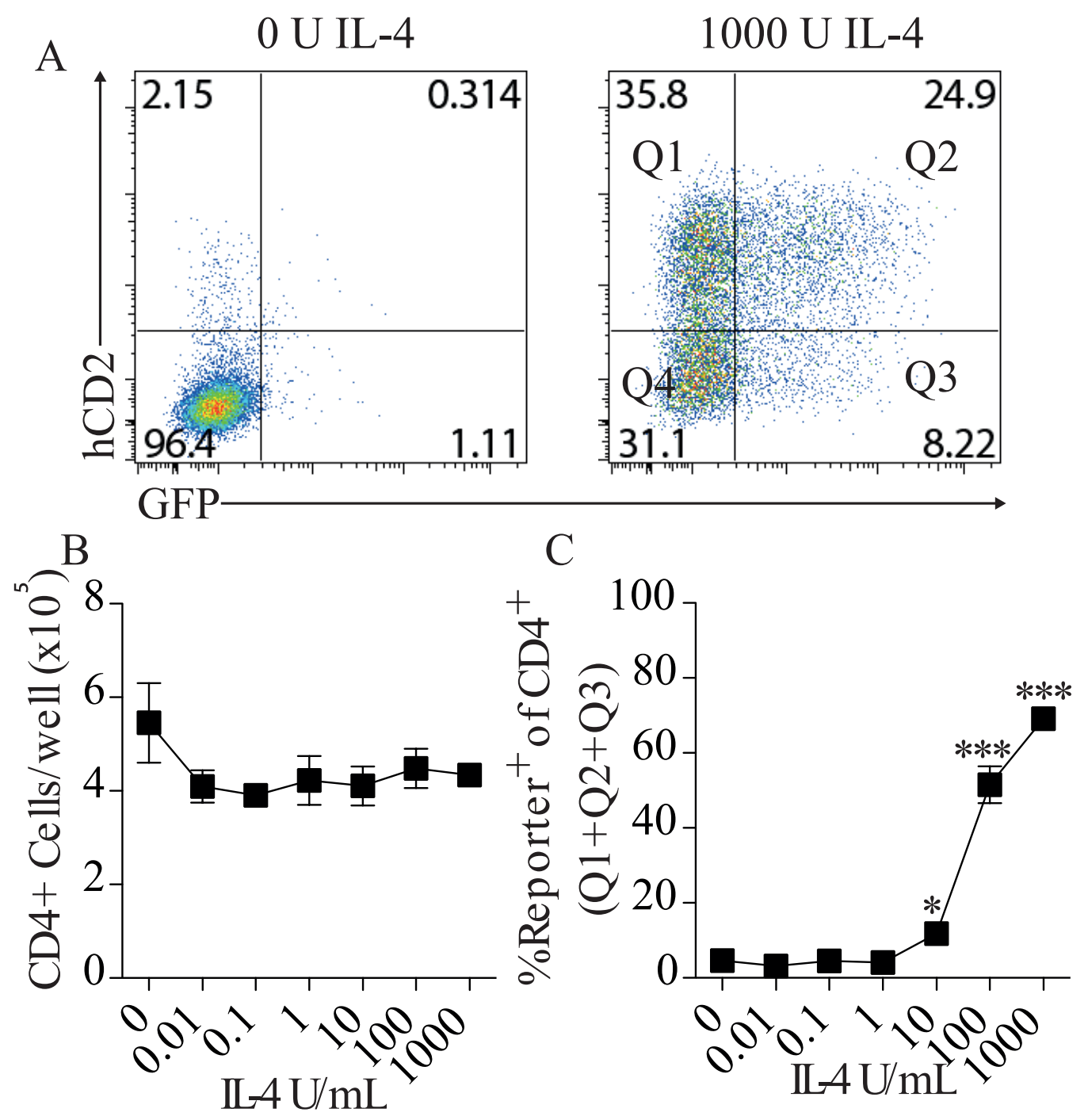

Figure 3.1: KN2/G4 cells cultured in IL-4 express IL-4 reporters. Splenocytes from KN2/G4 mice were stimulated on anti-CD3 coated plates for three days in the presence of $100 \mathrm{U} / \mathrm{mL}$ IL-2, anti-CD28 and titrating amounts of IL-4. (A) shows representative plots of cells cultured in the presence of 0 or 1000 units (U) of IL-4 and identifies quadrants 1-3 (Q1-3) used to define reporter-expressing cells. (B) The total number of live CD4+ cells and (C) proportion that were GFP+ and/or hCD2+ (Q1+Q2+Q3), were determined by trypan blue exclusion and flow cytometry. Data points show mean \pm SEM of $(n=3)$ individually cultured spleens from one of three similar experiments. Data were compared using one-way ANOVA with Bonferroni's post-test for increases above wells that did not contain IL-4; ${ }^{*} \mathrm{p}<0.05,{ }^{* * *} \mathrm{p}<0.001$. 


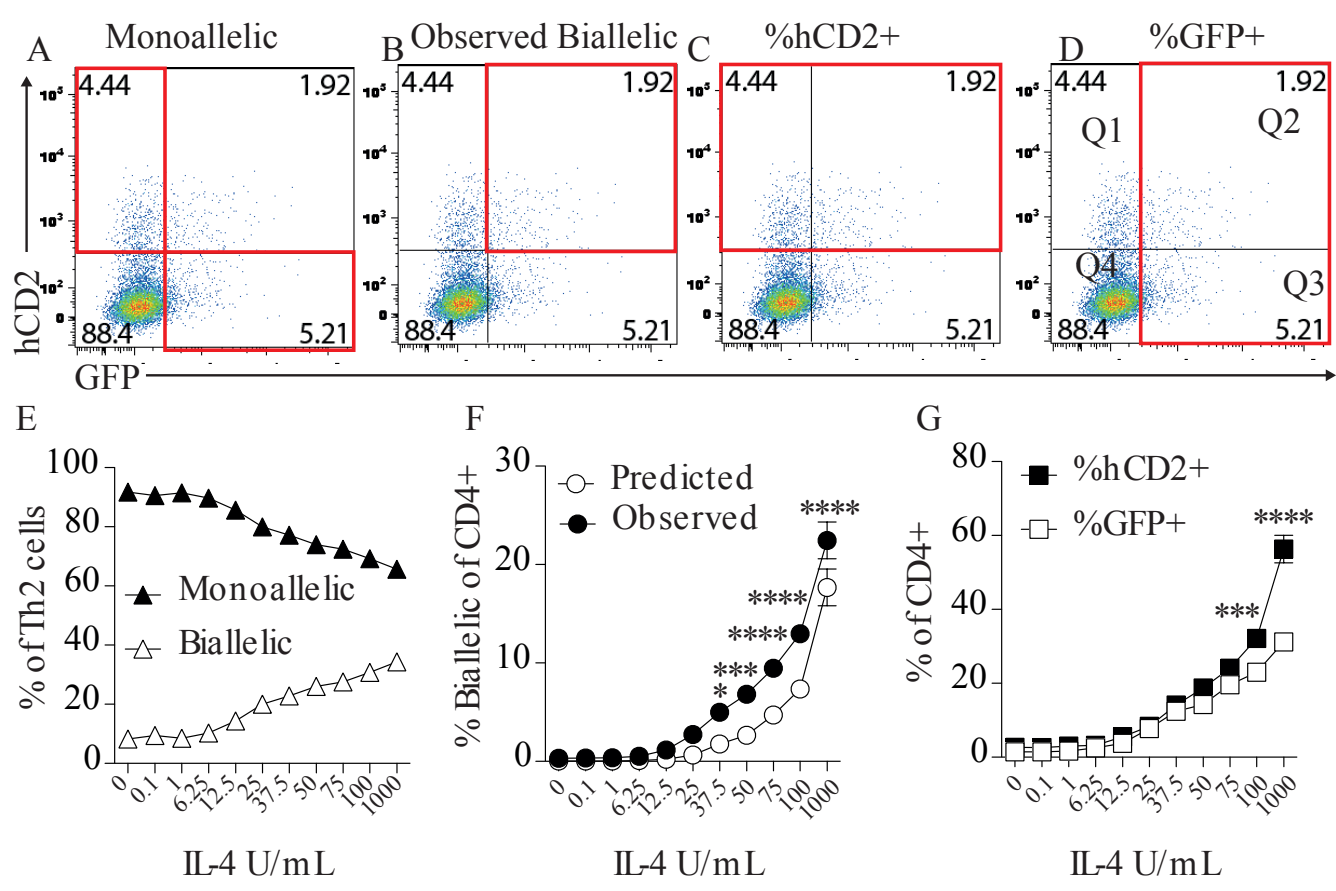

Figure 3.2: Culture in IL-4 increases biallelic IL-4 production above predicted levels. Cells were stimulated as in figure 3.1 for three days. The red boxes show the quadrants used to identify the total proportion of CD4+ T cells expressing (A) either hCD2 or GFP (monoallelic) (B) both hCD2 and GFP (biallelic), (C) hCD2, or (D) GFP. The same plot is shown in (A-D) and was generated in a culture containing $50 \mathrm{U} / \mathrm{mL}$ IL-4. (E) The total proportion of Th2 cells expressing IL-4 from one (monoallelic as in $(\mathrm{A})$ ) or both (biallelic as in (B)) alleles were compared; Data points were compared by two-way repeated measures ANOVA with Bonferroni's posttest and all data points were significant to $\mathrm{p}<0.0001$. (F) The proportion of CD4+ T cells expressing both hCD2 and GFP observed experimentally (black circles; as in (B)), or predicted by $\mathrm{P}_{\text {biallelic }}=\left(\% \mathrm{hCD} 2_{\text {total }}^{+} * \% \mathrm{GFP}_{\text {total }}^{+}\right) * 100$ (white circles, predicted), were compared. (G) The total proportion of CD4+ T cells expressing hCD2 (black; as in (C)) or GFP (white; as in (D)) were determined by flow cytometry. Data points show mean+SEM of $(n=3)$ individually cultured spleens from one of three similar experiments. Across experiments, both female and male mice were used and mice born to G4/G4 and KN2/KN2 mothers were assessed with similar differential expression profiles and biallelism responses irrespective of mouse gender or paternity. (F,G) Data were compared by Two-way repeated measures ANOVA with Bonferroni's post-test; ${ }^{*} \mathrm{p}<0.05,{ }^{* * *} \mathrm{p}<0.001,{ }^{* * * *} \mathrm{p}<0.0001$ at the same dose of IL-4. 


\subsubsection{Examining IL-4 production in differentiated Th2 cells}

We observed that primary culture in the presence of more than $25 \mathrm{U} / \mathrm{mL}$ IL- 4 resulted in an increased proportion of cells expressing hCD2 and GFP biallelically (Figure $3.2 \mathrm{~F}$ ) and were interested to determine if this biasing was maintained in imprinted Th2 cells. Th2 cells can remain stably polarised to the Th2 subset after the removal of IL-4 and TCR stimuli for at least 3-7 days[1, 343, 388]. Therefore, in the next set of experiments, our aim was to determine the allelic expression profile of rested Th2 cells upon reactivation.

In this experiment, KN2/G4 and +/+ cells were stimulated with anti-CD3/anti-CD28 in the presence of anti-IL-4 or 1000 U IL-4 for five days, and then transferred to uncoated culture wells containing anti-IL-4 and $100 \mathrm{U} / \mathrm{mL}$ IL-2. Three to four days later, cells were restimulated on anti-CD3-coated plates in the presence of anti-CD28 and IL-2 for 5-6 hours. While the frequency of reporter positive cells under Th0 conditions was low and these assays may have limited statistical reliability, when we examined the proportion of cells that expressed both hCD2 and GFP prior to restimulation, less than $10 \%$ of reporter+ cells expressed both alleles, under both anti-IL-4 and plus IL-4 culture conditions (Figure 3.3A and 3.3C). When cells were restimulated, we observed proportional increases in hCD2 and GFP expressing cells from cells cultured under both anti-IL-4 and plus IL-4 culture conditions (c.f. figure 3.3A and 3.3B). In cells cultured with anti-IL-4, while biallelic expression was four-fold higher than prior to stimulation, the ratio of mono:biallelic expression was unaltered (Figure 3.3C). In contrast, in IL-4-conditioned cells we observed a more pronounced 100-fold increase in biallelic expression, and an altered mono:biallelic expression ratio (Figure 3.3C). While the low frequencies of biallelic cells under anti-IL-4 culture conditions may be statistically limiting and will require follow-up validation, these data indicate that enrichment in biallelic expression was observed in restimulated IL-4-conditioned cells.

In an additional effort to examine the allelic expression profile of Th2-conditioned cells, we asked whether monoallelic expression predominated only in KN2/G4 cultures. Therefore, we examined the frequency differential of single or dual IL-4 allele expression in KN2/+ and G4/+ cultures (Figure 3.4). In these experiments, spleno- 
cytes were activated in the presence of IL- 4 for five days, subsequently rested for 5-6 days in the presence of anti-IL-4 and restimulated in the presence of anti-IL-4. Monensin was added two hours prior to harvest at each timepoint. At each timepoint, cultures were normalised such that hCD2 and GFP proportions were less than $0.1 \%$ in $+/+$ cultures, and the IL- $4+$ pouplation was less than $0.1 \%$ in $\mathrm{KN} 2 / \mathrm{G} 4$ cultures. This is why the gates in the figures differ at the two timepoints.

When we examined expression of hCD2 and GFP after three hours, the majority of Th2 cells expressed a single IL-4 allele in KN2/G4, KN2/+ and G4/+ cultures (Figure 3.4A-F). By six hours, the proportion of Th2 cells that were biallelic had increased in all three strains (Figure 3.4D-F). The biallelic population in KN2/G4 cultures plateaued at its maxima (Figure 3.4D); in KN2/+ and G4/+ cultures, IL-4 production was not maintained and resulted in a diminution of the biallelic population concomitant with the loss of IL-4 (Figure 3.4E and 3.4F). We compared the observed frequency of biallelism to the expected frequency as calculated previously (Figure $3.2 \mathrm{~F}$ ). In all three strains, statistically significantly for the KN2/+ and G4/+ strains at the six hour timepoint (peak of the response), the proportion of cells detected as biallelic was greater than predicted (Figure 3.4G-I). These studies indicate that while IL-4 production derived predominantly from a single allele, biallelism was more frequent than predicted. This was a feature of both primary and secondary Th2 cell stimulation. 


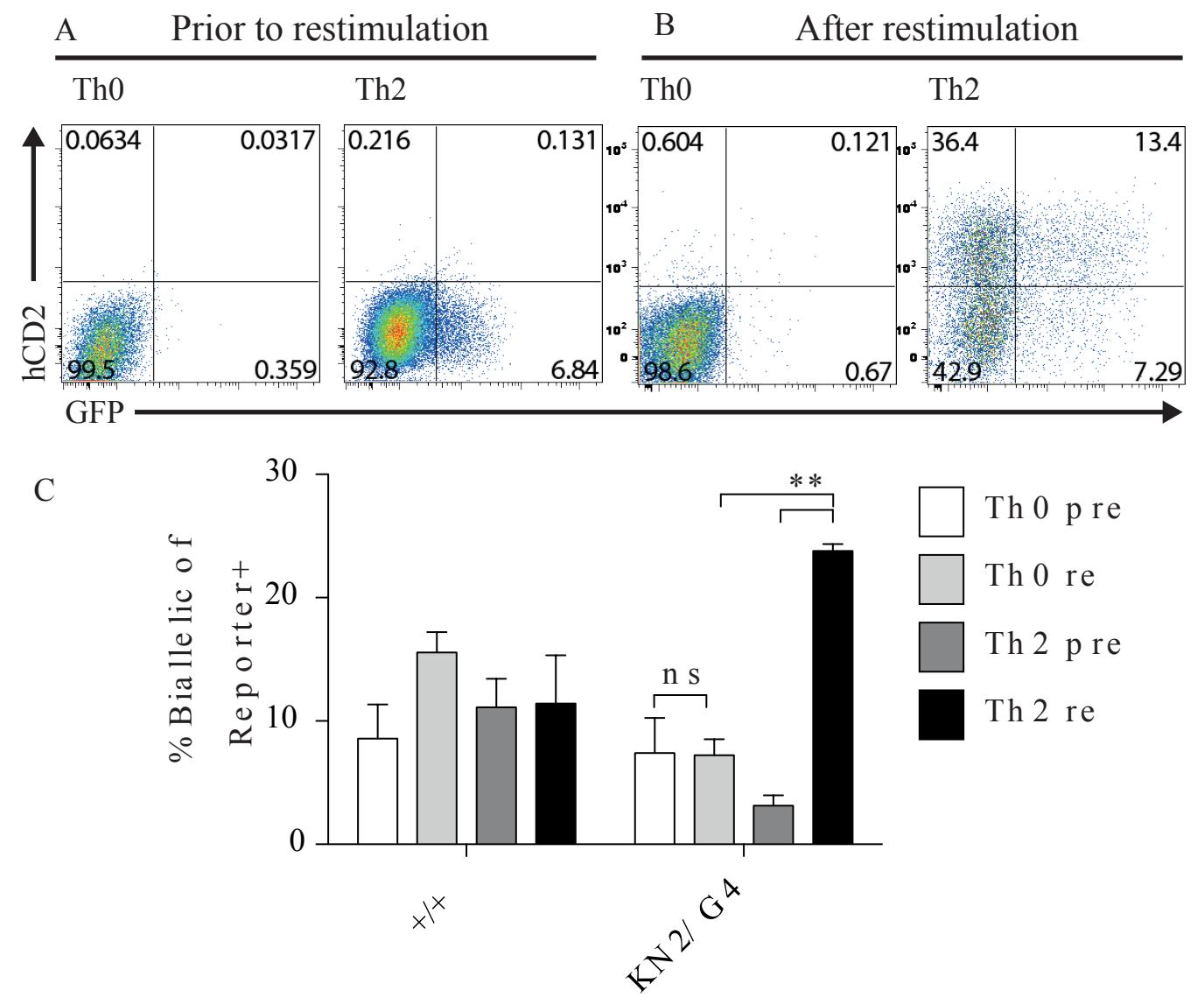

Figure 3.3: Culture in IL-4 potentiates cells to produce IL-4 biallelically upon secondary stimulation. After culture on anti-CD3-coated plates for five days in the presence of $100 \mathrm{U} / \mathrm{mL}$ IL-2, anti-CD28 and (Th0) anti-IL-4 or (Th2) $1000 \mathrm{U} / \mathrm{mL}$ IL-4, spleen cells were rested for three to four days in uncoated plates containing $100 \mathrm{U} / \mathrm{mL}$ IL-2 and then restimulated with anti-CD3 in the presence of anti-CD28 and $100 \mathrm{U} / \mathrm{mL}$ IL-2. (A) Cells were examined prior to or (B) 6 hours after restimulation. (C) The proportion of cells that expressed both hCD2 and GFP were compared in cells prior to (pre) and after (re) restimulation. Bars show mean \pm SEM of single spleens cultured in triplicate from one of two similar experiments. Data were analysed by Two-way ANOVA with Bonferroni's post-test; ${ }^{* *} \mathrm{p}<0.01$. 

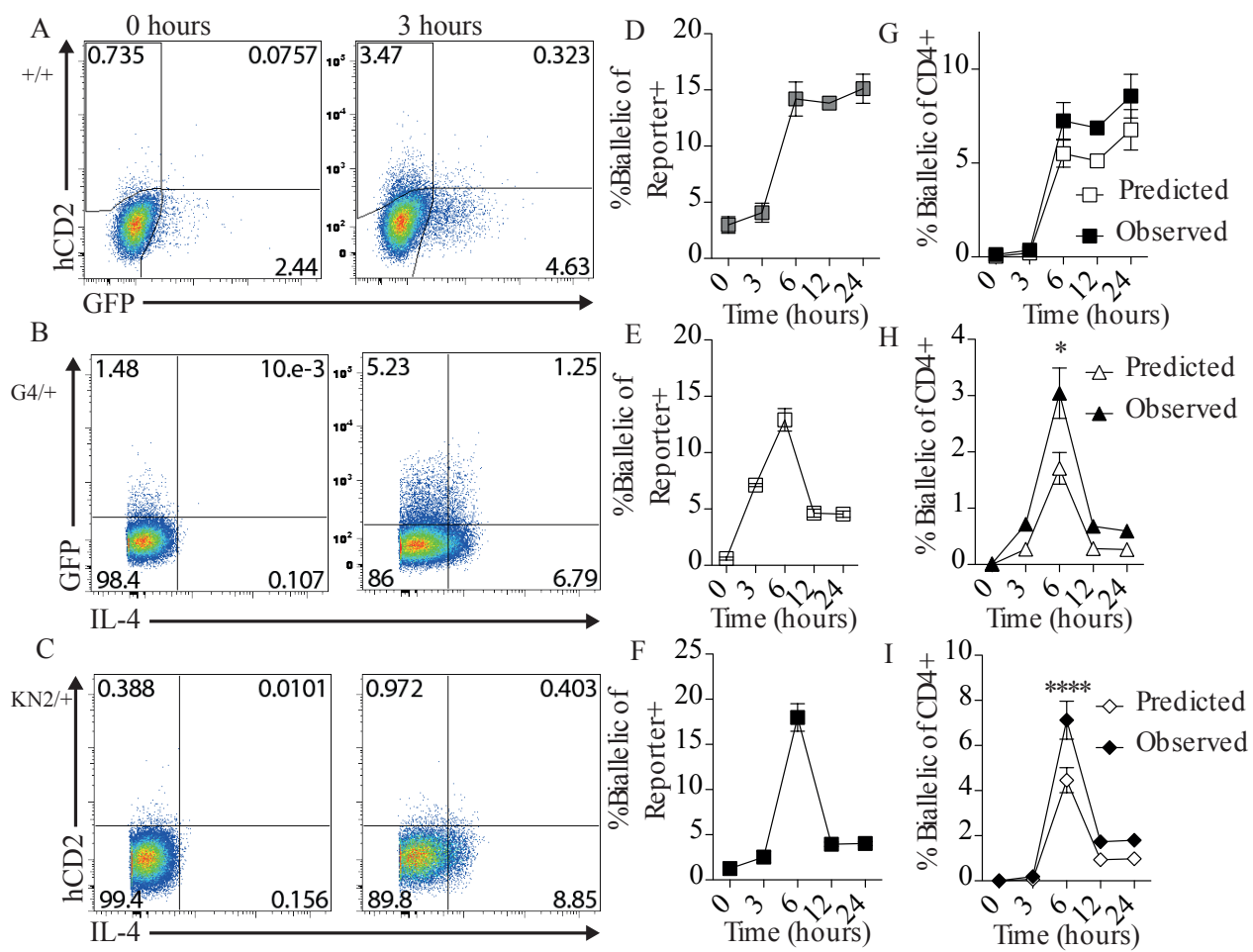

Figure 3.4: Biallelic IL-4 production exceeds predicted frequencies in restimulated KN2/+ and G4/+ cells. Splenocytes culture in the presence of 1000 $\mathrm{U} / \mathrm{mL}$ IL-4 as in Figure 3.3 were rested for five days. Cells were then left unstimulated or restimulated on anti-CD3 in the presence of $100 \mathrm{U} / \mathrm{mL}$ IL-2, anti-CD28 and anti-IL-4 and examined for hCD2, GFP and IL-4 production. Unstimulated cells were cultured with monensin for 2 hours and then stained, and all other cultures had monensin added for the last two hours of culture. (A) KN2/G4 (B) G4/+ and (C) $\mathrm{KN} 2 /+$ cells were examined for GFP, hCD2 and intracellular IL-4 without stimulation (0 hours) or after 3 hours ( 3 hours). The proportion of Th2 cells that were biallelic in (D) KN2/G4, (E) G4/+ and (F) KN2/+ cultures was determined. The predicted (determined as in Figure 3.2) and observed frequencies of CD4+ T cells that were biallelic were compared in $(\mathrm{G}) \mathrm{KN} 2 / \mathrm{G} 4,(\mathrm{H}) \mathrm{G} 4 /+$ and (I) KN2/+ cultures. Data show mean \pm SEM of $(n=2-3)$ individually cultured spleens from one of three similar experiments. (G-I) Data were compared using Two-way repeated measures ANOVA with Bonferroni's post-test; ${ }^{*} \mathrm{p}<0.05,{ }^{* * * *} \mathrm{P}<0.0001$. 


\subsubsection{Testing for interactions between IL-4, KN2 and G4}

In our studies, hCD2 expression was more frequent than GFP expression, and up to $75 \%$ of GFP + cells co-expressed hCD2 (Figure 3.1A). Therefore, we were interested to determine if hCD2 was out-competing the G4 allele for transcription factors, resulting in a skewed hCD2:GFP ratio as we had observed (Figure 3.2G). If increases in the expression from one allele were an impediment to the expression of the other, we predicted that IL-4 production by G4/+ and KN2/+ cultures would differ; similarly, GFP expression in KN2/G4 cultures would be different from G4/+ cultures, and likewise for hCD2 expression in KN2/G4 and KN2/+ cultures. We used Th2 conditioned spleen cells rested for 5-6 days, and then restimulated in the presence of anti-IL-4 to compare hCD2, GFP and IL-4 production (by intracellular cytokine staining) in the three different strains of mice. Monensin was added for the final two hours of culture. We also included wild-type $(+/+)$ mice in the experiments.

When we restimulated the cells, the proportion of cells producing IL-4 was similar in $\mathrm{KN} 2 /+$ and G4/+ cultures, and 50-65 percent of $+/+$ cultures (Figure 3.5A). Up to $30 \%$ of $+/+$ cultures produced IL- 4 , whereas the two heterozygous cultures peaked at $\approx 20 \%$. We also observed similar expression of hCD2 in KN2/G4 and KN2/+ cultures, and similar expression of GFP in KN2/G4 and G4/+ cultures (Figure 3.5B and C). These relationships were maintained at all timepoints examined after restimulation. The kinetics of IL-4 production varied between experiments, but in all cases, intracellular IL-4 expression was maximal between 6 and 12 hours, as shown (Figure 3.5A). Proportional hCD2 and GFP expression were maintained for much longer periods than intracellular IL-4, such that the proportion of cells expressing hCD2 or GFP plateaued and were maintained out to 24 hours, and this occurred similarly in IL4-sufficient and IL-4-deficient heterozygotes (Figure 3.5B and C). Thus, we conclude that while the absolute levels of hCD2 and GFP expression were different, this was not a result of transcription factor competition between the constructs. Therefore, while GFP and hCD2 expression were not independent events in IL-4-conditioned cells, neither were they cross-antagonistic. 


\subsubsection{Comparison of IL-4 production in homozygous and het- erozygous reporter strains}

Previous investigations examining the allelic expression profile of IL-2 in vitro compared IL-2+/+ and IL-2+/- cultures, and observed a 50-65\% differential between the wild-type and heterozygous cultures[402]. Our observation of a 50-65\% differential between $+/+$ and the two heterozygous strains (Figure 3.5A) lead us to ask if, despite being expressed to different absolute maxima, similar differentials may be observed between reporter heterozygotes and homozygotes. In the next experiments, we cultured cells in the presence of IL-4 or anti-IL-4, and examined hCD2 and GFP expression three days later. In these experiments, we used spleen cells from $+/+, \mathrm{KN} 2 /+, \mathrm{KN} 2 / \mathrm{KN} 2, \mathrm{KN} 2 / \mathrm{G} 4, \mathrm{G} 4 /+$ and G4/G4 mice. Quadrants for hCD2 and GFP were positioned to below $0.1 \%$ in $+/+$ cultures. Representative plots from which data were generated are shown in figure 3.6 and figure 3.7.

We began by examining hCD2 and GFP expression in cells cultured in anti-IL-4. After three days of primary culture in medium containing anti-IL-4, we observed that the proportion of KN2/G4 CD4+ T cells that expressed hCD2 was 50-65\% the frequency of KN2/KN2 cultures (Figure 3.8A). Similarly, when we examined GFP expression, we observed a similar proportional reduction in KN2/G4 cells compared to G4/G4 cells (Figure 3.8B). G4/+ and KN2/+ cultures demonstrated similar reductions, although these differences were not stastically significant. We conclude that similar relative decreases in hCD2 and GFP expression in heterozygous versus homozygous reporters occurred in the absence of IL-4 signalling.

When we examined hCD2 and GFP expression in our IL-4-containing cultures, the relationship was different. GFP expression in KN2/G4 cells remained close to the predicted range differential (50-65\% of G4/G4 mice; figure $3.8 \mathrm{D}$ ), but the addition of IL-4 induced almost equivalent frequencies of hCD2+ cells in KN2/G4 and KN2/KN2 cultures (Figure 3.8C). KN2/+ cultures expressed hCD2 at $80 \%$ the level of KN2/KN2 cultures and G4/+ cultures expressed GFP at $\approx 70 \%$ of G4/G4 cultures (Figure 3.8C and $3.8 \mathrm{D}$ ). These data may indicate that the KN2 allele is more responsive to IL-4 than the G4 construct, but this will require more investigation at a molecular level. Our studies from these experiments indicate that the behaviour of the KN2 and the 
G4 allele were similar under anti-IL-4 conditions, but may differ following culture in the presence of IL-4.

Collectively our experiments suggest that monoallelic expression of IL-4 predominates in KN2/G4 cultures. Our data also indicate that hCD2 and GFP reflect the production of IL-4 in vitro. We identify that the KN2 and G4 alleles are not crossantagonistic, and in the absence of IL-4, behave similarly to the wild-type IL-4 gene. Additionally, we observed biallelism more frequently than was predicted, which may indicate that biallelic IL-4 expression is a unique feature of a specialised population of Th2 cells. 

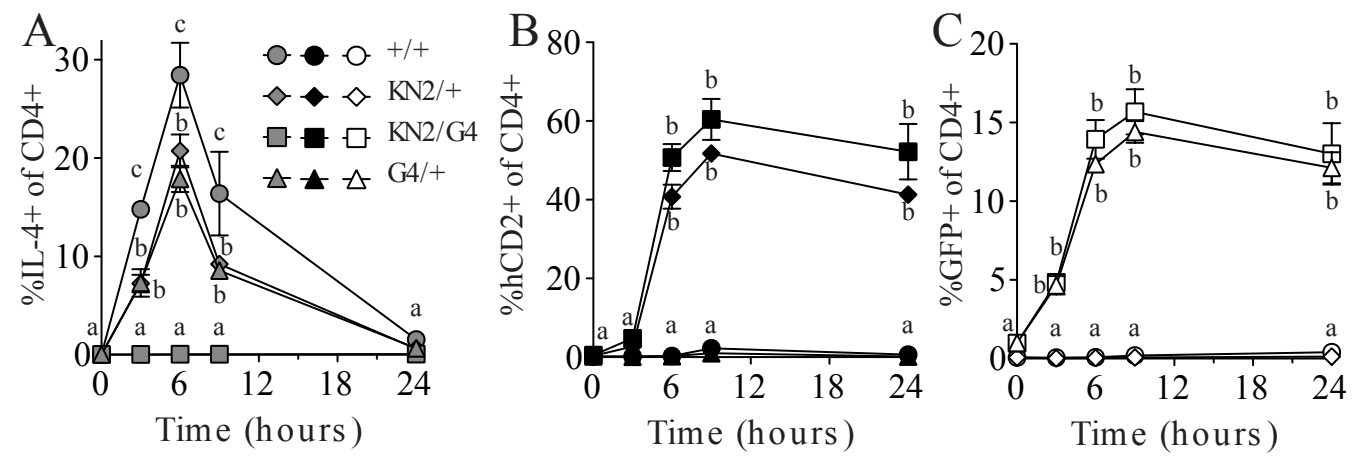

Figure 3.5: IL-4 production is equivalent in heterozygous reporter cells. Cells were treated as in Figure 3.4 and restimulated on anti-CD3-coated plates in medium containing anti-IL-4, anti-CD28 and $100 \mathrm{U} / \mathrm{mL}$ IL-2. Time zero wells were transferred to uncoated wells in the same medium and all wells had monensin added 2 hours before harvest, including the unstimulated (time zero) culture. Cells were stained intracelluarly and analysed by flow cytometry to determine live CD4+ T cell (A) IL-4 production, (B) hCD2 expression or (C) GFP expression after the stated times. Data points represent mean \pm SEM of $(n=3)$ individually cultured spleens from one of three similar experiments. Data were analysed by Two-way repeated measures ANOVA with Bonferroni's post-test. Different lowercase letters indicate statistically significant differences comparing groups at the same time points; $\mathrm{p}<0.05$. 


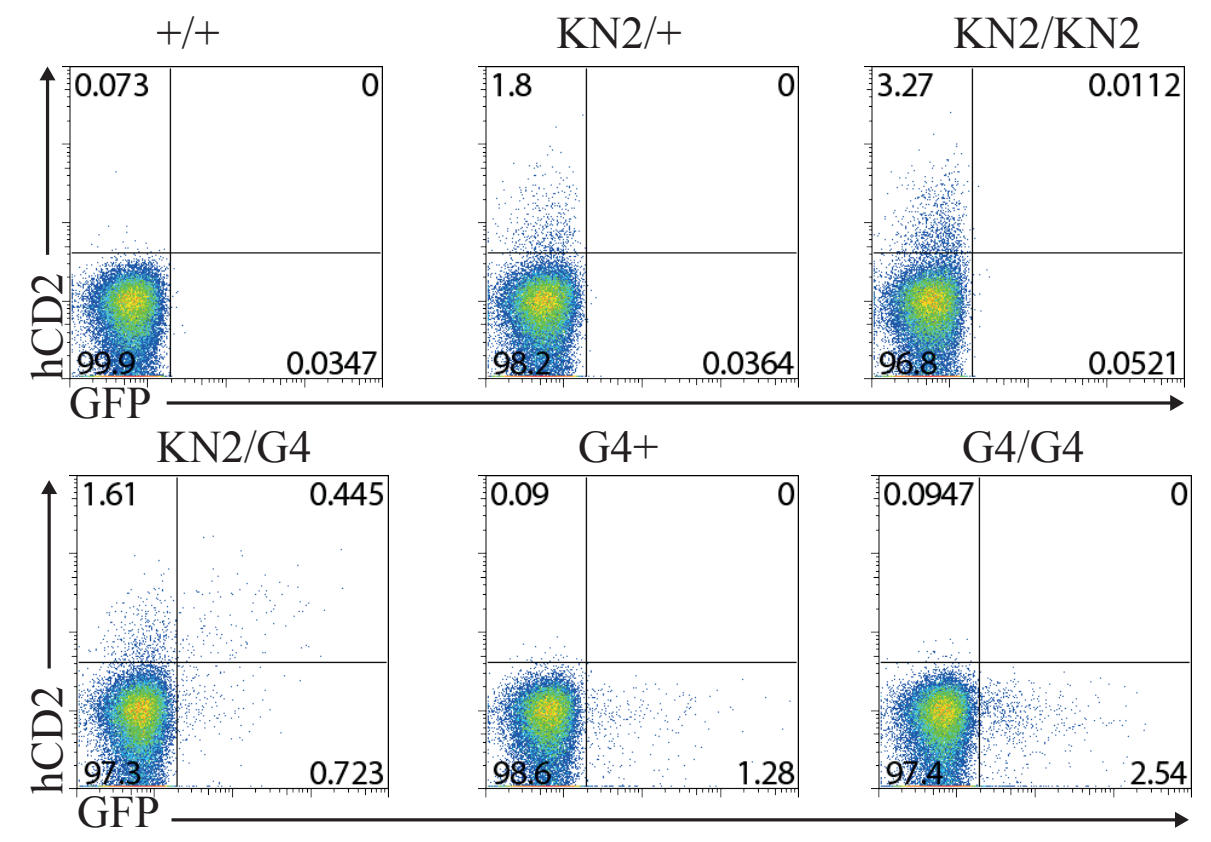

Figure 3.6: Reporter expression by homozygous and heterozygous reporter cells cultured in anti-IL-4. Cells from mice of the indicated genotype were cultured on anti-CD3-coated plates in medium containing $10 \mu \mathrm{g} / \mathrm{mL}$ anti-IL-4, anti-CD28 and $100 \mathrm{U} / \mathrm{mL}$ IL-2 for three days and examined for reporter expression by flow cytometry. Plots are shown from one experiment used to generate data in Figure 3.8. 


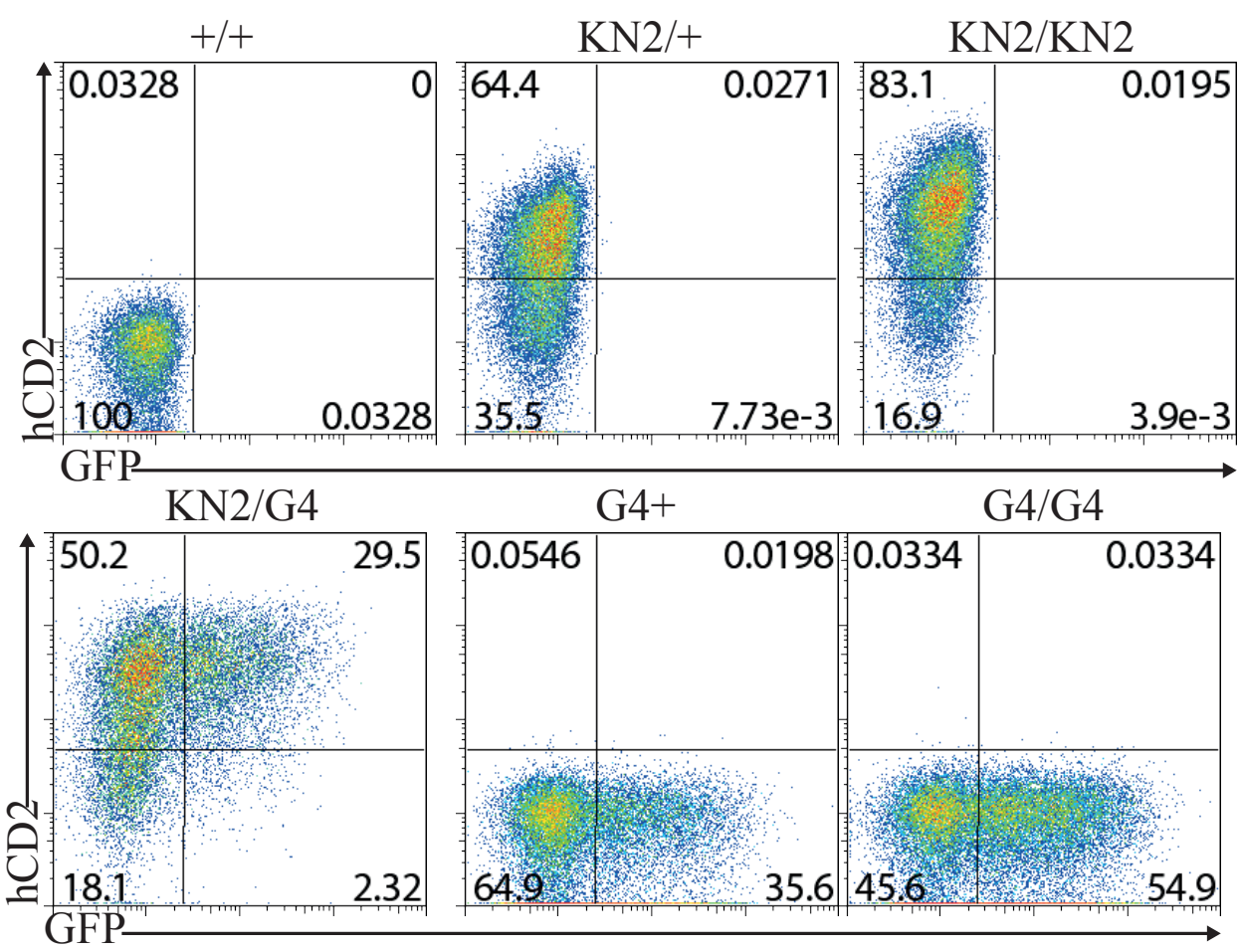

Figure 3.7: Reporter expression by homozygous and heterozygous reporter cells cultured in IL-4. Cells from mice of the indicated genotype were cultured on anti-CD3-coated plates in medium containing $1000 \mathrm{U} / \mathrm{mL}$ IL-4, anti-CD28 and 100 $\mathrm{U} / \mathrm{mL}$ IL-2 for three days and examined for reporter expression by flow cytometry. Plots are shown from one experiment used to generate data in Figure 3.8. 

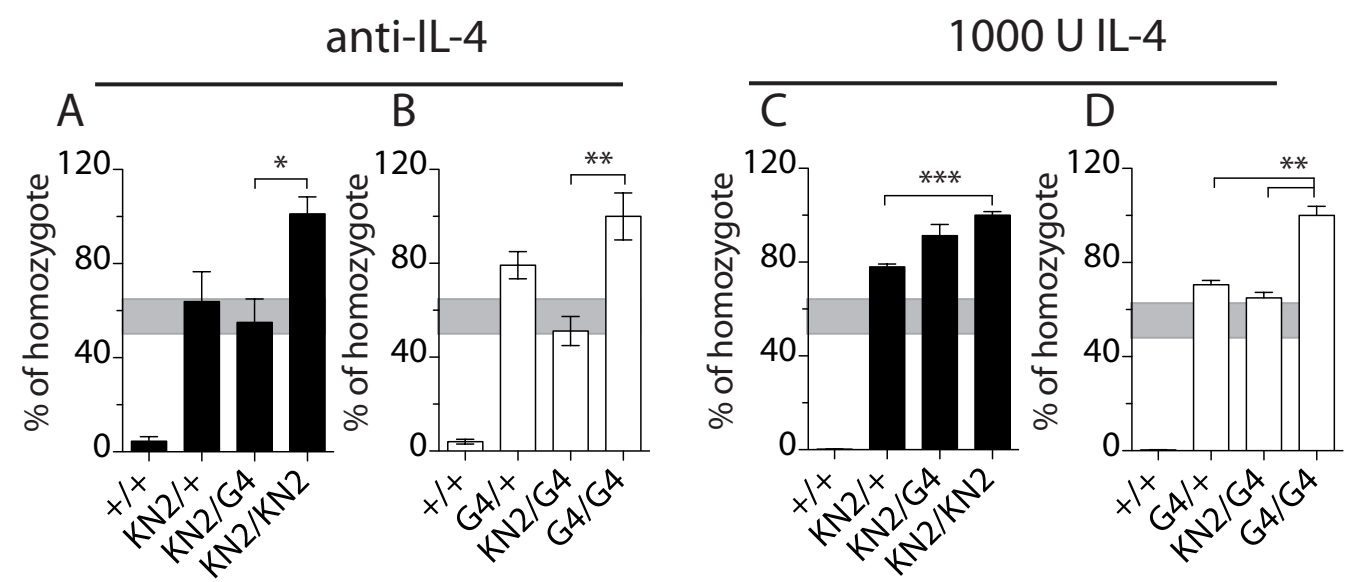

Figure 3.8: hCD2 and GFP behave similarly in the absence of IL-4 signalling. Total splenocytes received three days of primary anti-CD3/anti-CD28 stimulation in medium containing $10 \mu \mathrm{g} / \mathrm{mL}$ anti-IL-4, or medium containing $1000 \mathrm{U} / \mathrm{mL}$ IL-4 as indicated. CD4 + T cells were examined for hCD2 (black bars) or GFP (white bars) as gated in Figure 3.2C and 3.2D. Data were normalised relative to expression in $\mathrm{KN} 2 / \mathrm{KN} 2$ homozygotes $(\mathrm{A}, \mathrm{C})$ or $\mathrm{G} 4 / \mathrm{G} 4$ homozygotes (B, D). Data represent mean+SEM of $(n=7)$ anti-IL-4 and $(n=8)$ plus IL-4 individual spleen replicates combined from three (anti-IL-4) or four (plus IL-4) experiments. In each experiment anti-IL-4 cultured homozygote reporter expression was between 2 and 10\%, and IL-4 cultured homozygote reporter expression was 30 to $90 \%$ of total CD4+ T cells. The area between 50 and $65 \%$ is shaded in grey to reflect the predicted difference based on the difference in IL-4 production in restimulated IL-4-conditioned cells shown in figure 3.5. Data were analysed by Kruskall-Wallis with Dunn's post-test. ${ }^{*} \mathrm{p}<0.05$, ${ }^{* *} \mathrm{p}<0.01,{ }^{* * *} \mathrm{p}<0.001$. 


\subsection{Discussion}

The manner in which IL-4 production is regulated is an important focus of allergic disease biology. Over the past 15 years considerable advances in understanding have been made regarding the mechanisms that control its expression, conditions under which it is upregulated, and the epigenetic changes that stably maintain access to the Th2 locus to permit its production. One of the observations, now made by numerous groups, is that many IL-4-producing cells transcribe only one of their two IL-4 alleles. We have added to these studies and monitored allelic IL-4 production in primary and secondary in vitro culture settings in two reporter systems. We found that in KN2/G4 cells, most reporter-expressing cells expressed only one of the two reporter constructs. We observed that biallelism was more frequent, as a proportion of the Th2 cell population, in cells cultured in the presence of IL-4 than in its absence, and was also more frequent than we predicted for a gene with two independently regulated alleles. We further observed that, compared to wild-type, or reporter homozygote cultures, heterozygous cultures produced only 50-65\% as much IL-4. Taken together, these studies support the concept that monoallelic IL4 gene expression is a frequent feature of Th2 cells.

\subsubsection{Probabilistic monoallelic regulation of IL-4}

Monoallelic IL-4 production has been observed numerous times in the literature. It was first reported by Bix and Locksley[389]. These authors demonstrated that approximately $50 \%$ of Th2 clones had transcripts that were detectable from only one of two alleles; this was a stable, heritable phenotype and could be transcribed from either allele[389]. These were followed by studies from the same group demonstrating that the proportion of CD4+ $\mathrm{T}$ cells producing IL-4, rather than the amount of IL-4 produced per cell resulted in biased IL-4 production by BALB/c compared with C57BL/6 CD4+ T cells[390]. Subsequently, studies in two separate reporter mice demonstrated that IL-4 was expressed predominantly monoallelically. Rivière et al.[374] used a precursor reporter to the KN2 system, and observed, for the most part, two distinct populations of hCD2-expressing or IL-4-producing cells[374]. How- 
ever, they also demonstrated that the strength of TCR mediated signalling could increase the proportion of cells that were biallelic, under both anti-IL-4 and plus IL-4 conditions[374]. These early findings indicated that the strength of stimulus, in this case TCR stimulation, could enhance biallelic expression of IL-4. Hu-Li et al.[273] added to these works and investigated the longeivity of monoallelic expression. By cloning individual $\mathrm{T}$ cells, they found that irrespective of the mono- or bi-allelic status of a given cell, when passaged multiple times, the allelic expression profile of a population of daughter progeny would recapitulate the original profile[273]. These findings indicated that while there was clone-to-clone variation in the likelihood of expressing one, other or both alleles, for a given outgrown clone, the probability of expression was a predictable event.

The clearest illustration of monoallelic IL-4 production came from subsequent work by Guo, Hu-Li \& Paul[381]. Guo et al.[381] used RNA fluorescence in situ hybridisation to analyse IL-4 expression on a per-allele basis[381]. In these studies, 538 cells expressing IL- 4 were examined, and only $15.4 \%$ of these cells were expressing IL-4 from both alleles[381]. When cells expressing only one of the two IL-4 alleles were examined, there was a strong change in histone acetylation at an IL-4 enhancer, but only on the favoured allele[381]. There is an overwhelming body of literature that establishes the potential for monoallelic expression of IL-4 in vitro, and strong arguments that attribute this to a probabilistic regulatory process[273, 381, 388-390, 403].

\section{Advantages of allele-specific IL-4 production}

The next point to consider is why such a process would evolve. Generally, natural selection results in the accumulation of beneficial traits. IL-4 can have major deleterious effects if it is produced inappropriately[404, 405]; control of IL-4 production through probabilistic regulation has been argued as a potentially advantageous regulatory mechanism[381, 390, 401]. In this respect, probabilistic rather than deterministic regulation could provide tighter control of IL-4-dependent events; the example of $B$ cell IgE class-switch recombination and macrophage type 2 activation were proposed by Guo et al., because controlling the number of IL-4-producing cells, rather than the amount produced by an individual cell, would make it easier to balance the strength- 
of-stimulus with an appropriate strength of outcome ([381, 401]; reproduced in figure 3.9). In light of recent data indicating that in vivo, IL-4 can induce STAT-6 phosphorylation in all non-Th2 cells in an inflamed lymph node[334], one additional context in which probabilistic regulation could be conceivably advantageous is in limiting the frequency with which IL-4-exposed T cells begin to produce IL-4. The germinal centre of the lymph node contains high frequencies of IL-4-producing TFH cells[275, 362], which means germinal centre TFH cells are likely to be surrounded by IL-4 producing cells in high frequency. Plausibly, a probabilistic regulatory mechanism could prevent exponential amplification of IL-4 production through a positive feedback loop acting on the IL-4 producing cells in close proximity to each other. This does, however, require that IL-4 influences Th2 cell development in vivo, which is currently a controversial topic. However, there are potential advantages in regulating IL-4 production in a probabilistic process that can result in the expression of protein from only a single allele.

In this context, the question arises, why do we see biallelic cells? In our studies we saw that there was variability in the expression profile of each allele because some cells in the KN2/G4 system expressed only one allele, but a reasonable proportion expressed both alleles (Figure 3.6 and 3.8). As IL-4 expression is thought to result from probabilistic regulation in which access to each allele is conferred independently[401] it is reasonable to infer that biallelic cells arose from two independently regulated, but not mutually exclusive, individually expressed alleles. In part, biallelic expression supports the concept of a probabilistic monoallelic regulation mechanism. One of the attributes of probabilistic regulation is that it can result in a bimodal expression pattern[387, 388]: Genes are either expressed or they are not. For hCD2 and GFP we observed reasonably bimodal populations (Figure 3.6 and 3.8). In earlier work, bimodal expression of IL-4 was observed[388] and in some of our experiments we observed sufficient separation of the IL-4+ and IL-4- populations to suggest that IL-4, too, was expressed in a similar manner. Thus the presence of the biallelic population does not preclude a probabilistic regulatory mechanism that acts at the allele-specific level from operating for IL-4. However, we also observed that biallelic IL-4 expression occurred at higher-than-expected frequencies (Figures 3.2 and 3.4), which may indicate that biallelic expression reflects a small predisposition among the 
Th2 cell population to express both IL-4 containing chromosomes simultaneously and this point deserves some specific discussion, as follows.

\section{Is there evidence of functional subtypes of Th2 cells based on their allelic expression profile?}

It is important to consider potential reasons that underlie the higher-than-expected occurrence of biallelic IL-4-producing cells. In our studies, we observed that biallelism was increased by exposure to IL-4; previous studies have demonstrated that biallelic IL-4 expression can be increased if the level of TCR stimulation is increased[374]. It may be that once signal strength reaches a certain threshold, biallelism becomes more likely, which could explain the data and relate our findings to those of Rivière et al.[374]. This may provide the cells with a particular niche, and provides the exciting possibilty that biallelic cells are a specific functional subset of Th2 cells. An alternative explanation relates to the differentiation of the Th2 population. As a population, Th2 cells that have divided more than four times produce on average more IL-4[347, 376]. Biallelism may be enriched in cells at a certain stage of development, or in cells that have proliferated a set number of times. This could be addressed by examining biallelism in cells labelled with proliferation dyes at the start of the experiment. STAT6-deficient cells have also been shown to develop IL-4-producing capacity and this is associated with eventual accumulation of increased levels of GATA-3[348]. Examining GATA-3 levels in KN2/G4 cells may be a simple means to identify a signal that is associated with biallelic expression. It may also relate to pre-exisiting Th2 cells that were present in our culture at the start. Our experiments used whole spleen cells from naive mice, but cells with mixed surface phenotypes were present in these cultures; it is possible that the higher-than-expected biallelic frequency is due to a small number of in vivo-generated pre-existing Th2 cells in which IL-4 production is regulated in a different manner to the bulk cultured population. Using sorted naive populations in future experiments could be used to address this appropriately. Further, in this light, investigation of the biallelic potential of Th2 cells in vivo may identify novel aspects of Th2 cell development that are important for allergic responses and protection from parasites. 


\section{Reporter independence}

The independent regulation of each reporter observed in our in vitro experiments (figure 3.5) suggests that cross-antagonism of reporters is unlikely to occur. When we examined KN2/+ and G4/+ cultures, we observed that these cells produced equivalent amounts of IL-4 (Figure 3.5). These findings indicate that G4 and KN2 were equally permissive of IL-4 production. Further, the behaviour of each reporter, relative to homozygotes was similar under anti-IL-4 conditions (Figure 3.8). Comparing the absolute frequencies of hCD2 or GFP+ Th2 cells in KN2/G4, KN2/KN2 and G4/G4 mice may allow us to gain a preliminary view of the influence of a single allele on IL-4 production in vivo. The disparity in absolute expression, however, requires further discussion.

The insertion sites of the KN2 and G4 reporter constructs were similar, but not identical. The KN2 reporter allele replaces the first two exons, and the intronic region between the two genes. Similarly, the G4 allele replaces the first intron and 178 base pairs of the subsequent intronic region of the gene[273]. Differences in these knockin sites may inadvertently influence the success of their transcription. Second, the KN2 reporter included a CD4 enhancer cassette, which was inserted to amplify its expression and improve detection[373-375]. This may improve the likelihood of KN2 transcription without interfering with the counterpart allele. Examining similarly sized mRNA (cDNA) products for KN2 and G4 may allow us to determine whether there are differences in the amount of transcription, that could contribute to the differential reporter expression. Similarly, examining mRNA stability with the actinomycin D stability assay[406] may allow us to compare the two constructs and see if they are equivalently stable, which could also influence their absolute expression. These or similar investigations will be informative in future studies to explain the differences in absolute expression of the two constructs.

Collectively, our investigations have established the behaviour of the KN2/G4 reporter system in vitro. These data provide evidence of the utility of the mouse for in vivo studies of Th2 immunity. Our studies indicate that the majority of IL-4 is derived from a single allele in vitro, and provide some novel directions for future work. Future experiments will be required to determine how IL-4 expression is regulated in 
vivo. 

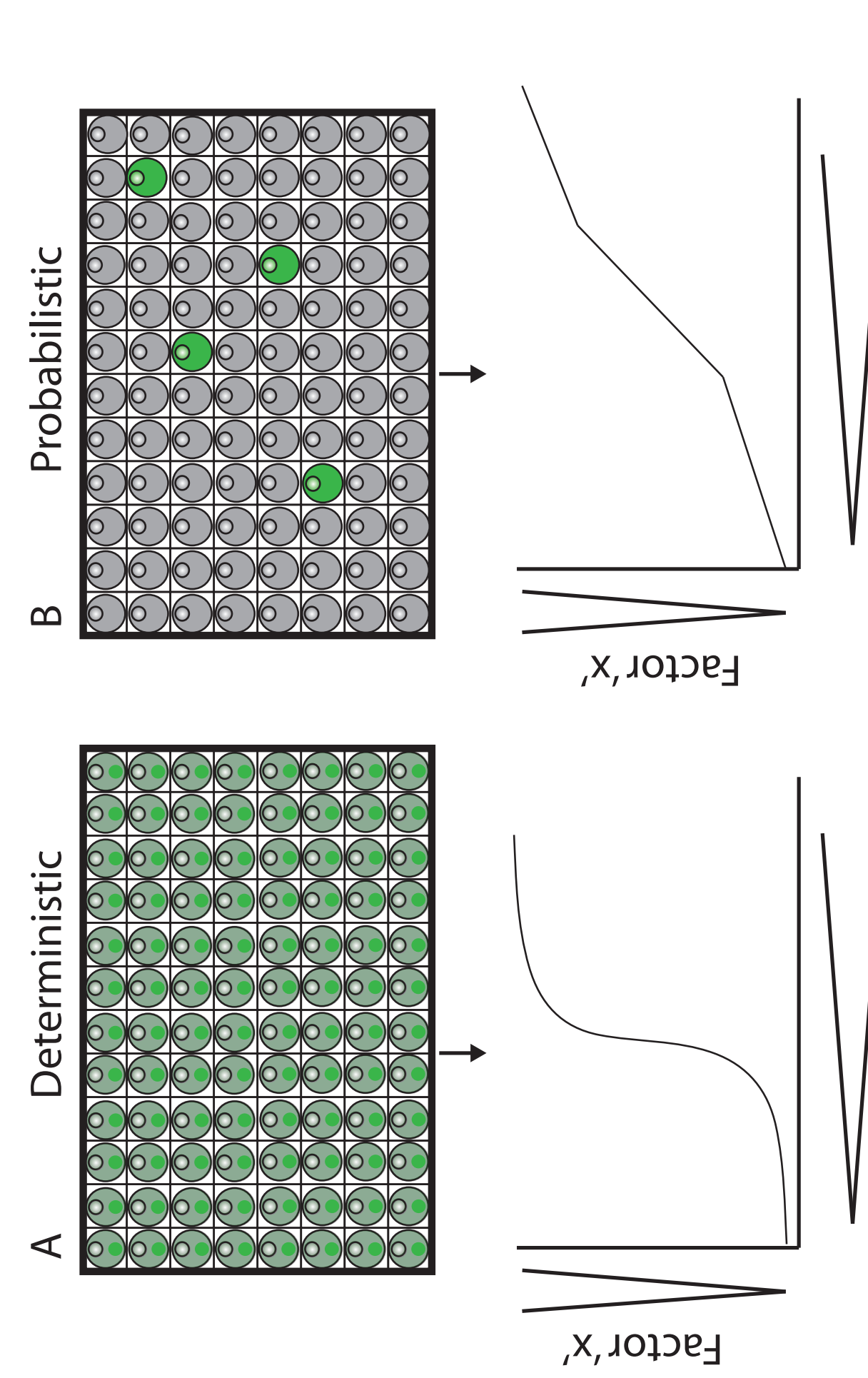

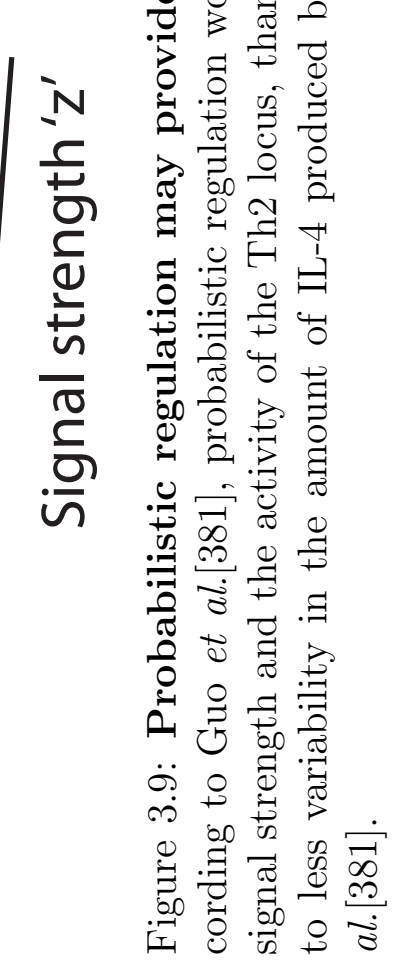




\subsection{Summary}

We find that the majority of IL-4-producing cells in vitro express only a single IL-4 allele. Biallelic IL-4 production was more frequent in IL-4-conditioned cells. Higherthan-expected frequencies of cells producing IL-4 biallelically than is predicted for independently regulated alleles were observed. Expression from one IL-4 allele does not influence expression from the counterpart allele; these events are independent events, but are induced by the same stimuli. 


\section{Chapter 4}

\section{Evaluating the contribution of IL-4 to Th2 cell development and function in vivo}

\subsection{Introduction}

\subsubsection{Establishing how genetic lesions in IL-4 affect allergic Th2 cell development and function in vivo}

The development of IL-4-producing CD4+ Th2 cells is a critical aspect of the allergic process. One of the key factors that promotes Th2 cell development in vitro is exposure to IL-4. Studies have investigated the role of IL-4 in in vitro Th2 cell development in great depth, however, there is limited information addressing whether, and to what extent, IL-4 is required for in vivo Th2 cell development. Understanding the cascade of events that leads to the development of an allergen-responsive Th2 cell in vivo may identify new ways to subvert the allergic process before it can manifest as disease.

There is evidence that IL-4 is not required for in vivo Th2 cell development. Prior to the advent of IL-4 reporter mice, it was shown that if anti-IL-4 antibody was injected before a primary $N$. brasiliensis infection, IgE production was impaired by greater than 99\%[288]. However, if IL-4 production was not blocked during a secondary infection of these same mice, $\mathrm{IgE}$ was produced faster and with greater magnitude than was observed during a primary infection[288]. As IgE production induced by secondary $N$. brasiliensis infection is CD4 $+\mathrm{T}$ cell dependent[407] and IL-4 dependent[289], these early findings suggested that Th2 cells can develop without IL-4 signaling. 
Previous studies in our lab investigated the role of IL-4 signaling in in vivo Th2 cell development more directly[1]. These studies employed an IL-4 reporter mouse, in which the gene encoding GFP is inserted under the control of the IL-4 promoter[273]. This is useful because IL-4 has a very short half-life and cannot be detected directly ex vivo. In this manner, IL-4-producing CD4+ T cells, which are typically classified as 'Th2 cells' $[324,325]$, can be identified directly ex vivo[1]. In the work done by our group, G4/+ mice, which remain IL-4-sufficient through the '+' allele, were compared to IL-4-deficient G4/G4 double reporter mice, or to G4/+ mice deficient in STAT6 signalling. This was done because STAT-6 is key for IL-4R $\alpha$ signalling[329-331], and is known to be important for optimal Th2 cell development in vitro[329]. The observations are outlined briefly below.

In response to live parasite infection, $\operatorname{IgE}$ generation and bronchoalveolar eosinophil influx were absolutely dependent on IL-4 and STAT-6, but no requirement for IL-4 or STAT-6 in the generation of Th2 cells in vivo was identified. In G4/+ and G4/G4STAT-6-/- mice, Th2 cells developed with similar kinetics; the induction of IL-5 and IL-13 mRNA in Th2 cells was similar in IL-4 deficient and sufficient mice; and memory Th2 cell expansion was similar in IL-4 sufficient and deficient mice[1]. These, and other findings, provide evidence that Th2 cells can develop in the absence of IL-4. A small number of other studies have assessed the role of IL-4 in Th2 cell development in vivo, however, none have examined the role of IL-4 in Th2 cell development to allergen in an adjuvant free context[1,360-363, 408]. Additionally, a limited number of studies have attempted to modulate the level of IL-4. Instead, they generally examine Th2 cell function in IL-4-deficient mice. Therefore, we were interested to closely evaluate the involvement of IL-4 in allergen-induced Th2 cell development and function in vivo.

In addition to the G4 reporter, an additional system that has been used to record Th2 cells by marking IL-4 producing cells, is the KN2 reporter mouse[373]. In this reporter, the gene encoding a non-functional version of the human CD2 gene replaces IL-4 but remains under the control of the IL-4 promoter[373]. In this reporter mouse, Th2 cells have also been observed to develop in vivo in the absence of IL-4[275]. In the studies described in this chapter, we have used both reporters, and additionally crossed homozygous KN2/KN2 and G4/G4 mice to generate KN2/G4 reporter mice. These 
KN2/G4 mice are IL-4 deficient, and can be used to examine Th2 cells expressing a single allele of IL-4. In the previous chapter we characterised the behaviour of the KN2/G4 mouse in vitro. In this chapter, we employ IL-4 reporter systems, including the KN2/G4 mouse, to ascertain how IL-4 contributes to Th2 cell development, and subsequently, Th2 cell function.

\subsection{Aims}

Our specific aims were:

1) To test whether IL-4 contributes to primary Th2 cell generation following adjuvantfree allergen immunization;

2) To determine how the lack of one IL-4 allele affects allergen-specific Th2 celldependent responses. 


\subsection{Results}

\subsubsection{Testing whether IL-4 is required for in vivo Th2 cell development}

The study by van Panhuys et al.[1] and other studies[275, 360] indicate that in vivo Th2 cell development does not require IL-4. Our first aim was to confirm these previous findings. We immunised $\mathrm{G} 4 /+$ and G4/G4 mice with 600 dead $i$ L3 $N$. brasiliensis larvae. Seven days after intradermal immunization, we observed similar proportional shifts, and similar 1000-fold increases in the numbers of Th2 cells in immunized G4/+ and G4/G4 lymph nodes (Figure 4.1B and C). Therefore Th2 cells indeed developed in the absence of IL-4 and to similar levels in G4/+ and G4/G4 mice. These data recapitulate previous findings[1] and indicate that there is no absolute requirement for IL-4 in Th2 cell development in vivo.

In this experiment, we observed that similar numbers of Th2 cells developed in G4/G4 mice and G4/+ mice (Figure 4.1C). G4/G4 mice have two reporter alleles, but G4/+ mice have only one. If IL-4 does not contribute to Th2 cell generation, Th2 cell numbers should have been more frequent in G4/G4 mice compared to G4/+ mice. Therefore, it is possible that there is a partial effect of IL-4 on Th2 cell development. Further, this experiment may have been underpowered to reveal a difference. However, it is not possible to distinguish between IL-4-dependent monoallelic IL-4 production and IL-4-independent biallelic production by comparing these mice. Therefore, an alternate system was required to distinguish between these two mechanisms.

In our in vitro studies, we determined that each allele in a KN2/G4 mouse was activated independently. The two alleles were induced by the same stimulus, but neither inhibited the level of expression from the counterpart IL-4 allele (Figure 3.5AC). Therefore, the KN2/G4 mouse provides an IL-4-deficient, single-allele reporter mouse, which would allow us to distinguish between IL-4-dependent and -independent Th2 cell development. 
A

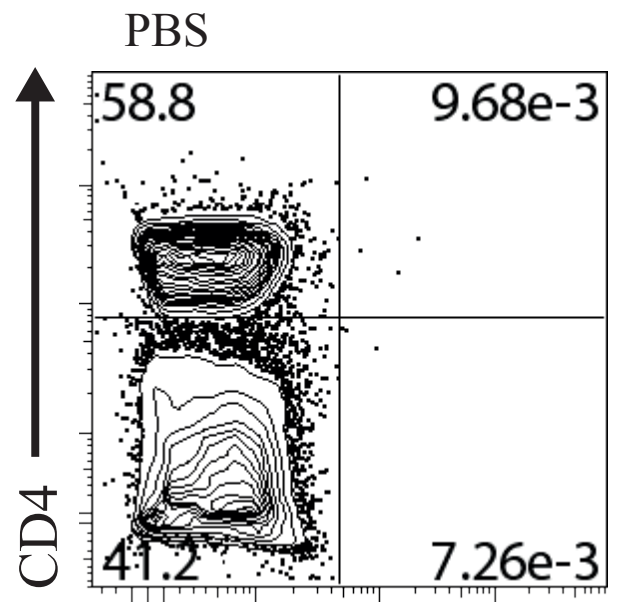

N.brasiliensis

\section{G4/GFP}

B
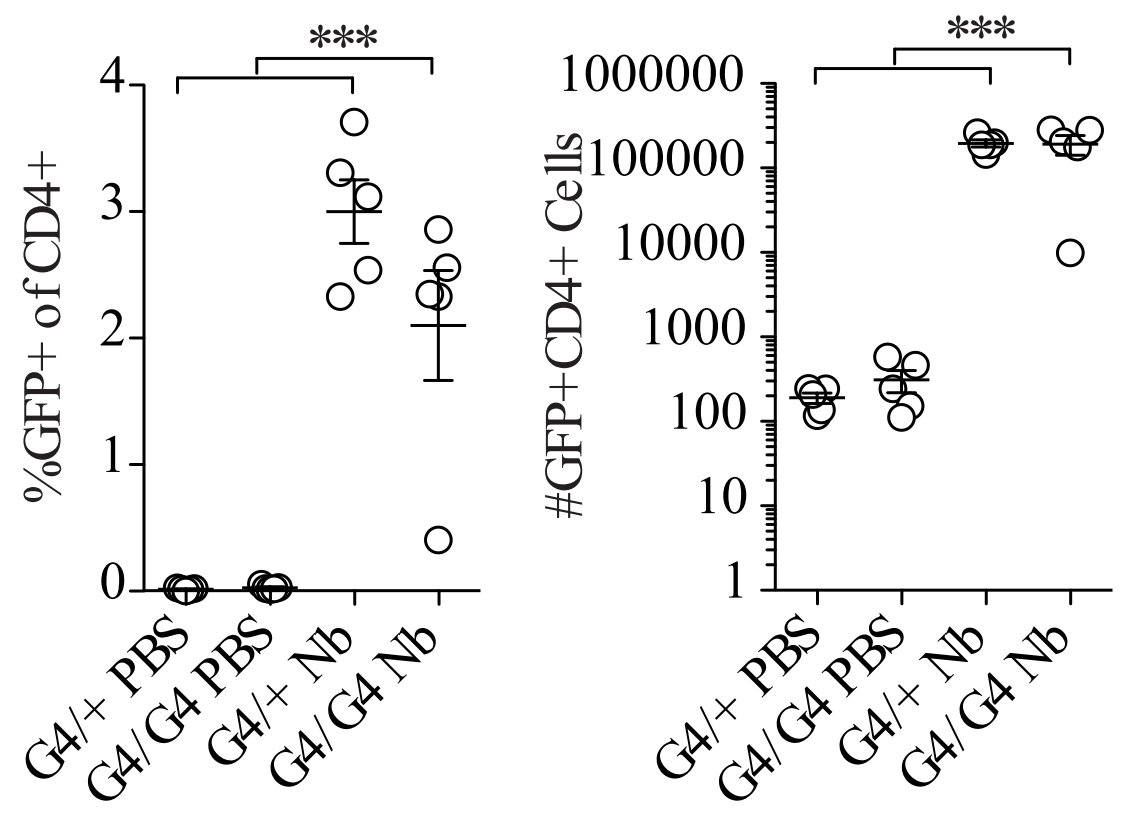

Figure 4.1: Th2 cells develop in the absence of IL-4 in vivo. G4/G4 or G4/+ mice ( $\mathrm{n}=5$ /group) were injected intradermally with 600 dead $i$ L3 $N$. brasiliensis larvae or $30 \mu \mathrm{L}$ PBS. Seven days later draining lymph nodes were excised and live cells were examined for GFP expression by flow cytometry. (A) Flow plots show CD4 and GFP expression in live-gated cells from PBS- or dead N. brasiliensis-immunized G4/G4 mice. (B) The proportion of CD4+ T cells expressing GFP was evaluated by flow cytometry. (C) Total GFP+CD4+ numbers were calculated by multiplying the proportion of live cells that were GFP $+\mathrm{CD} 4+$ by the total live cell count, as determined by trypan blue exclusion. Data points represent individual mice, and lines represent group mean \pm SEM, from one of four similar experiments. Data were compared using One-Way ANOVA with Tukey's post-test; *** $\mathrm{p}<0.001$. 


\subsubsection{Evaluating the influence of IL-4 on primary, adjuvant- free in vivo Th2 cell development}

To use the KN2/G4 reporter in our investigations, we next examined the Th2 response of single-allele reporter mice, that were either IL-4-sufficient or IL-4-deficient. In these studies, we used GFP+CD4+ T cell numbers to identify Th2 cells when comparing G4/+ and KN2/G4 mice; we used hCD2+CD4+ T cell numbers to identify Th2 cells when comparing $\mathrm{KN} 2 /+$ and $\mathrm{G} 4 /+$ mice.

We immunised G4/+, KN2/G4 and G4/G4 mice with $100 \mu \mathrm{g}$ HDM and examined the development of Th2 cells 3, 7 and 10 days later. We also examined Th2 cell numbers in naive mice on day 0 . Prior to injection on day 0 , we observed similar, low level, baseline numbers of Th2 cells in G4/+, KN2/G4 and G4/G4 strains (Figure 4.2B and $4.2 \mathrm{C}$ ). Three days after intradermal injection of $100 \mathrm{ug}$ HDM, we observed that the proportion and number of GFP $+\mathrm{CD} 4+\mathrm{T}$ cells in $\mathrm{G} 4 /+$ and KN2/G4 mice was similar, and approximately 50-60 percent of that observed in G4/G4 mice (Figure 4.2B and $4.2 \mathrm{C}$ ). When we examined the response seven and ten days after immunization, the proportion and number of GFP+CD4+ cells in G4/+ mice was higher than in KN2/G4 mice (Figure 4.2B). Therefore, in the presence of IL-4, Th2 cell development and/or survival was increased. We also noted that the proportional difference in Th2 cell numbers between G4/G4 and KN2/G4 mice was maintained on day seven, and the same trend held for day ten (Figure 4.2B and 4.2C). Further, while there was some variability at early timepoints, it may be important to note that the GFP median fluorescence intensity generally correlated with the relative proportion of GFP + cells observed. In all cases, on day 10 the median fluorescence intensity of GFP in the $\mathrm{KN} 2 / \mathrm{G} 4$ cells trended toward being lower than those from the respective G4/+ mice (Appendix Figure 8.3).

We next examined the response of $\mathrm{KN} 2 /+, \mathrm{KN} 2 / \mathrm{G} 4$ and $\mathrm{KN} 2 / \mathrm{KN} 2$ mice. Similar, almost identical observations were made in the KN2 reporter system. We observed that KN2/+ lymph nodes contained more hCD2+CD4+ T cells than KN2/G4 nodes on day ten (Figure 4.3B and 4.3C), and the average numbers of hCD2+CD4+ T cells detected in KN2/G4 lymph nodes were lower than numbers detected in KN2/KN2 nodes at all timepoints. We found that these differences were statistically significant 
on days three and seven (Figure 4.3B and 4.3C). The median fluorescence intensity of hCD2 also trended toward being lower on KN2/G4 cells in all day 10 experiments (Appendix Figure 8.3). Therefore, in both the G4 and KN2 reporter settings, IL-4 sufficiency increased the number of detectable Th2 cells induced by HDM after day three.

We considered it important to clarify the role of IL-4 in the Th2 response to parasite antigens. We intradermally immunised $\mathrm{G} 4 /+, \mathrm{KN} 2 / \mathrm{G} 4$ and $\mathrm{KN} 2 /+$ mice with 600 $i$ L3 $N$. brasiliensis larvae and examined the draining lymph node response 7 days later. When we compared hCD2 expression in $\mathrm{KN} 2 / \mathrm{G} 4$ and $\mathrm{KN} 2 /+$ mice, hCD2+ cells were more frequent in $\mathrm{KN} 2 /+$ lymph nodes (Figure $4.4 \mathrm{D}$ and $4.4 \mathrm{E}$, middle panels). We observed that GFP+ cells were more frequent in $\mathrm{G} 4 /+$ than $\mathrm{KN} 2 / \mathrm{G} 4$ mice (Figure 4.4D and 4.4E, left panels). When we determined the total number of Th2 cells expressing one, other, or both IL-4 reporter alleles, both the proportion and number of Th2 cells present in KN2/G4 lymph nodes was lower than in G4/+ or KN2/+ heterozygotes (Figure 4.4D and 4.4E, far right panels). Therefore, Th2 cell development was increased in the presence of IL-4 when parasite antigens were used as the immunogen. Further, when we examined the allelic expression profile of IL-4 in KN2/G4 mice, we observed that approximately 25 percent of Th2 cells expressed both hCD2 and GFP (Figure 4.4A). Therefore, Th2 cells in KN2/G4 mice were able to simultaneously express both IL-4 reporter alleles. 


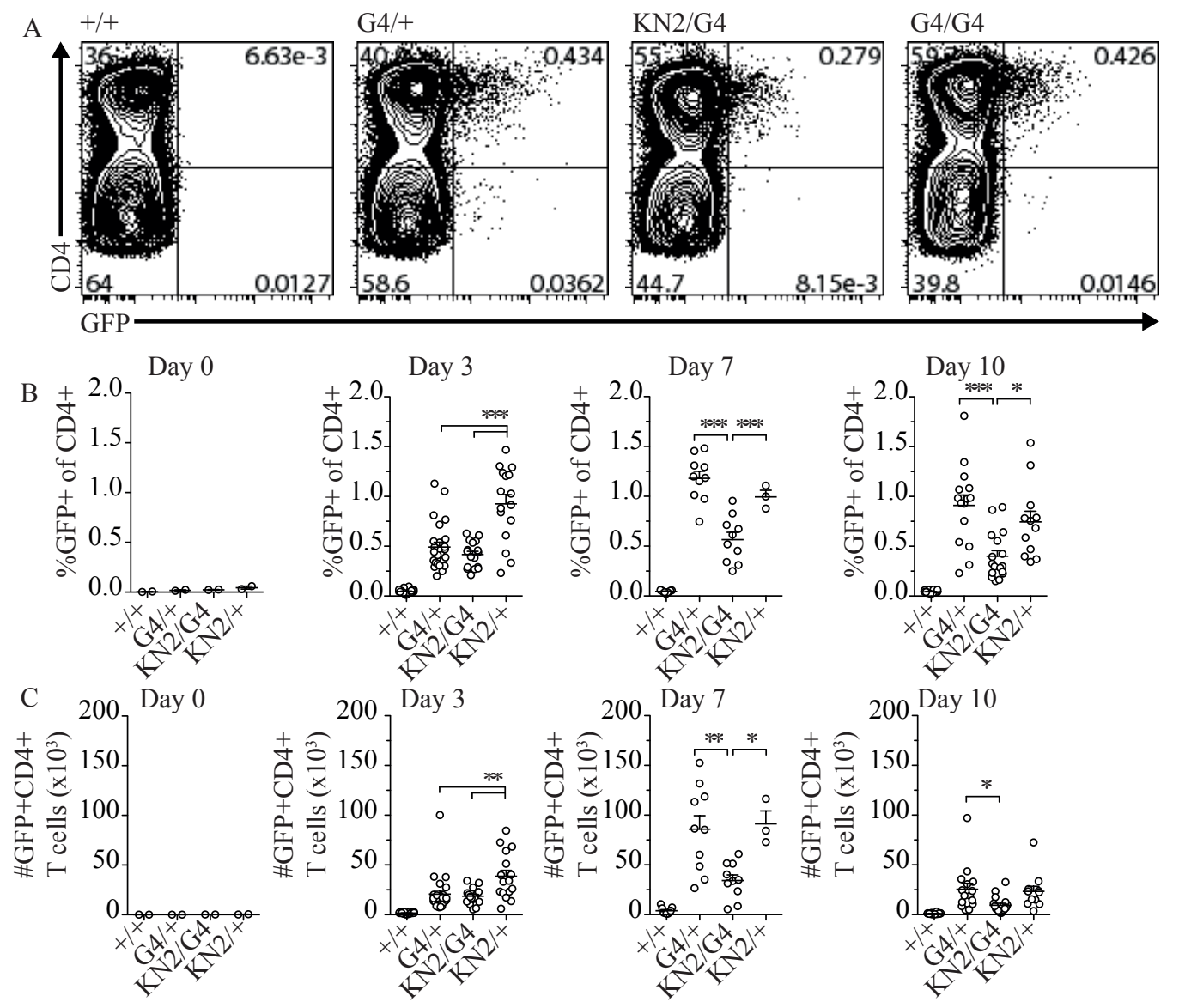

Figure 4.2: IL-4-independent and dependent phases of Th2 development revealed in IL-4 reporter mice. Mice were intradermally immunized with $100 \mathrm{ug}$ HDM and examined for CD4 and GFP expression by flow cytometry 3, 7 or 10 days later. (A) Shows representative plots of total live cell CD4 and GFP expression from mice of each indicated strain. (B) The proportion of CD4+ T cells expressing GFP and $(\mathrm{C})$ total number of $\mathrm{GFP}+\mathrm{CD} 4+\mathrm{T}$ cells in draining lymph nodes were compared. Data points represent individual mice, and lines represent group mean $\pm \mathrm{SEM}$; for Day 3 and 10, of $n=11-25$ mice combined from 3-5 experiments; for Day seven $n=9$ 10 mice combined from two experiments, with the exception of G4/G4 mice $(n=3$, one experiment). Data were normalised such that the GFP + population was $<0.05$ percent of total CD4 $+\mathrm{T}$ cells in $+/+$ mice at each timepoint. Data were compared using One-way ANOVA with Tukey's post-test; ${ }^{*} \mathrm{p}<0.05,{ }^{* *} \mathrm{p}<0.01,{ }^{*} *{ }^{*} \mathrm{p}<0.001$. 


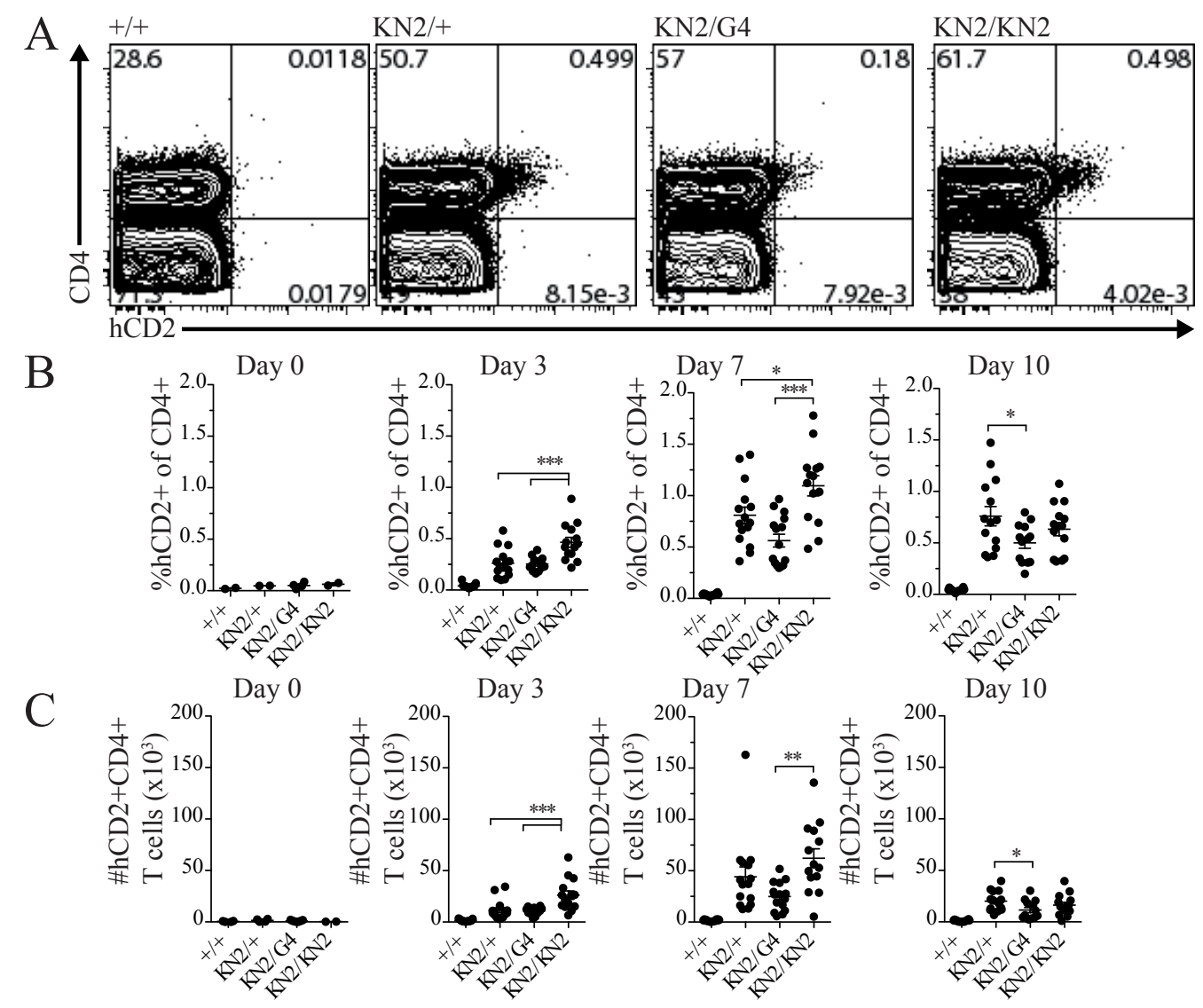

Figure 4.3: IL-4-independent and dependent phases of Th2 development confirmed in alternate reporter mice. Mice were intradermally immunized with 100 ug HDM and examined for CD4 and hCD2 expression 3, 7 or 10 days later. (A) Shows representative plots of total live cell CD4 and hCD2 expression from mice of each indicated strain. (B) The proportion of CD4+ T cells expressing hCD2 and (C) total number of hCD2+CD4+ T cells in draining lymph nodes were compared. Data points represent individual mice, and lines represent group mean \pm SEM; $n=9-15$ mice combined from 2 to 3 experiments at each timepoint. Data were normalised such that the hCD2 + population was $<0.05$ percent of total CD4 $+\mathrm{T}$ cells in $+/+$ mice at each timepoint. Data were compared using One-way ANOVA with Tukey's post-test; ${ }^{*} \mathrm{p}<0.05,{ }^{* *} \mathrm{p}<0.01,{ }^{* * *} \mathrm{p}<0.001$. 

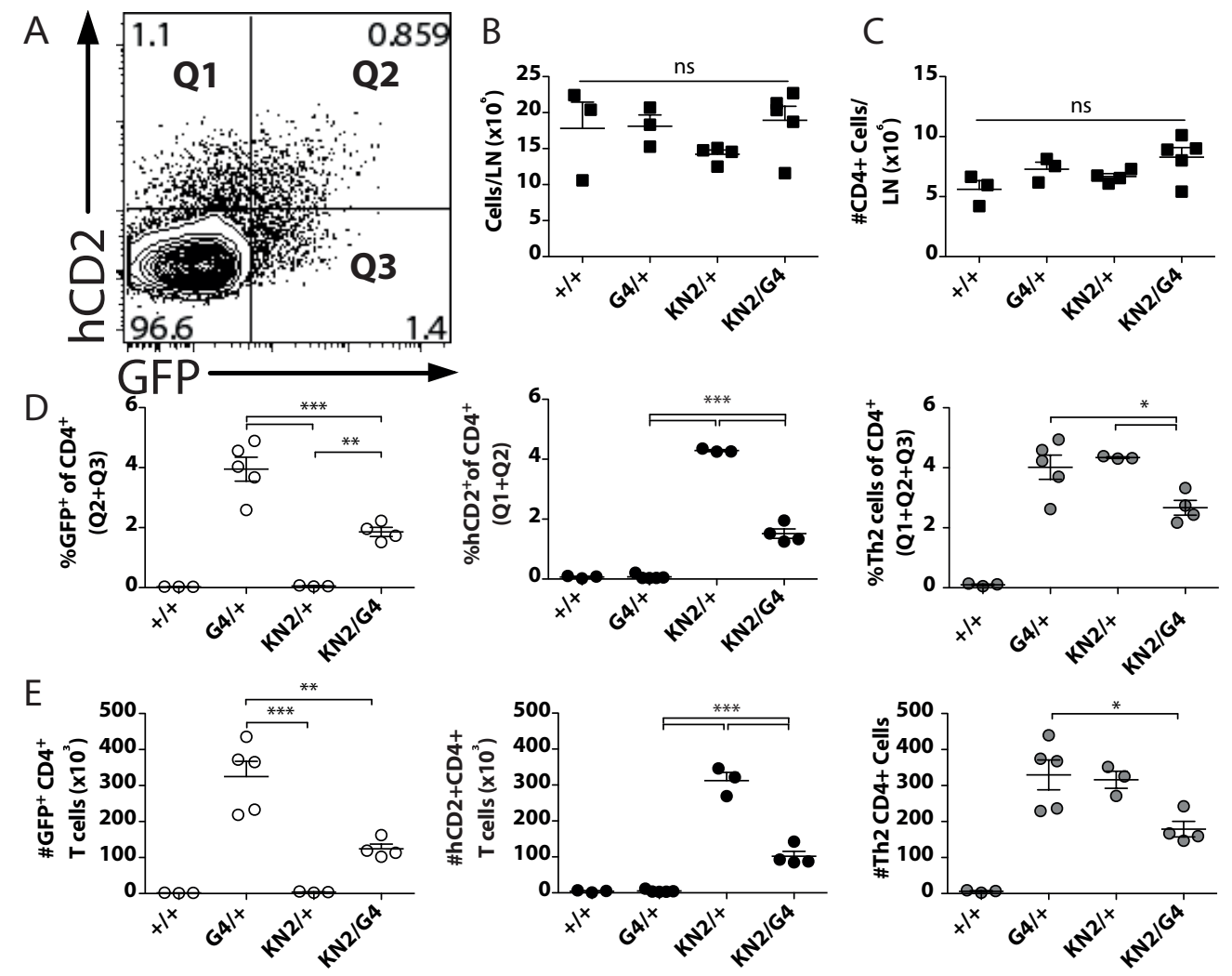

Figure 4.4: IL-4 enhances Th2 response to parasite allergens. Six hundred dead $i$ L3 $N$. brasiliensis larvae were injected into the dermal ear flap of mice of the indicated genotype and 6 days later hCD2 and GFP expression in live CD4+ T cells was examined by flow cytometry. (A) For each genotype, the proportion of cells in each quadrant was used to generate proportions and numbers shown in (D) and (E). (B) Total and (C) CD4+ T cell numbers isolated from draining lymph nodes of mice of the indicated genotype were quantified. (D) The proportion and (E) number of CD4+ cells expressing GFP (left), hCD2 (middle) and GFP and/or hCD2 (right) was assessed. Data points represent individual mice and lines represent group mean \pm SEM, $\mathrm{n}=3-5$ mice from one of two similar experiments. Data were compared using One-Way ANOVA with Tukey's post-test; ${ }^{*} \mathrm{p}<0.05,{ }^{* *} \mathrm{p}, 0.01,{ }^{* * *} \mathrm{p}<0.001$. 


\subsubsection{Establishing the role of STAT-6 in primary, adjuvant- free in vivo Th2 cell development}

STAT-6 is important for IL-4R signaling[329-331, 347]. As a final means by which to test the influence of IL-4 on Th2 cell development, we compared Th2 cell development in G4/+ and G4/+-STAT-6-/- mice. Based on the impairment we observed in our comparisons of KN2/G4 mice and IL-4 sufficient heterozygotes, we predicted that Th2 cells would be less frequent in G4/+-STAT-6-/- than in G4/+ lymph nodes. In this experiment, we immunised $\mathrm{G} 4 /+$ and G4/+-STAT-6-/- mice intradermally with $100 \mu \mathrm{g}$ HDM and examined Th2 cell development in the draining lymph node 7 days later. When we compared Th2 cell development in G4/+ and G4/+-STAT-6-/mice, we observed similar numbers of CD4+ T cells (Figure 4.5A), but Th2 cells were less frequent in the G4/+-STAT-6-/- mice (Figure 4.5B and 4.5C). Naive G4/+ and G4/+-STAT-6-/- lymph nodes contained similar numbers of CD4+ T cells and Th2 cells (Figure 4.5D-F). Therefore, Th2 cell development in response to primary HDM immunization was impaired in the absence of STAT-6 signaling.

Taken together, these data suggest that IL-4 signaling was not required for early Th2 cell development, but IL-4 signaling through STAT-6 amplified in vivo Th2 cell development. IL-4, therefore, contributed to Th2 cell generation over and above the ability of antigens to initiate Th2 cell development. Furthermore, the proportional reduction in hCD2-expressing Th2 cells in KN2/G4 mice compared to KN2/KN2 mice, in GFP-expressing Th2 cells in KN2/G4 mice versus G4/G4 mice and the observation that approximately 75 percent of cells expressed only one of the two reporters in KN2/G4 mice, also suggests that biallelic IL-4 expression in vivo occurs, but is rare. 

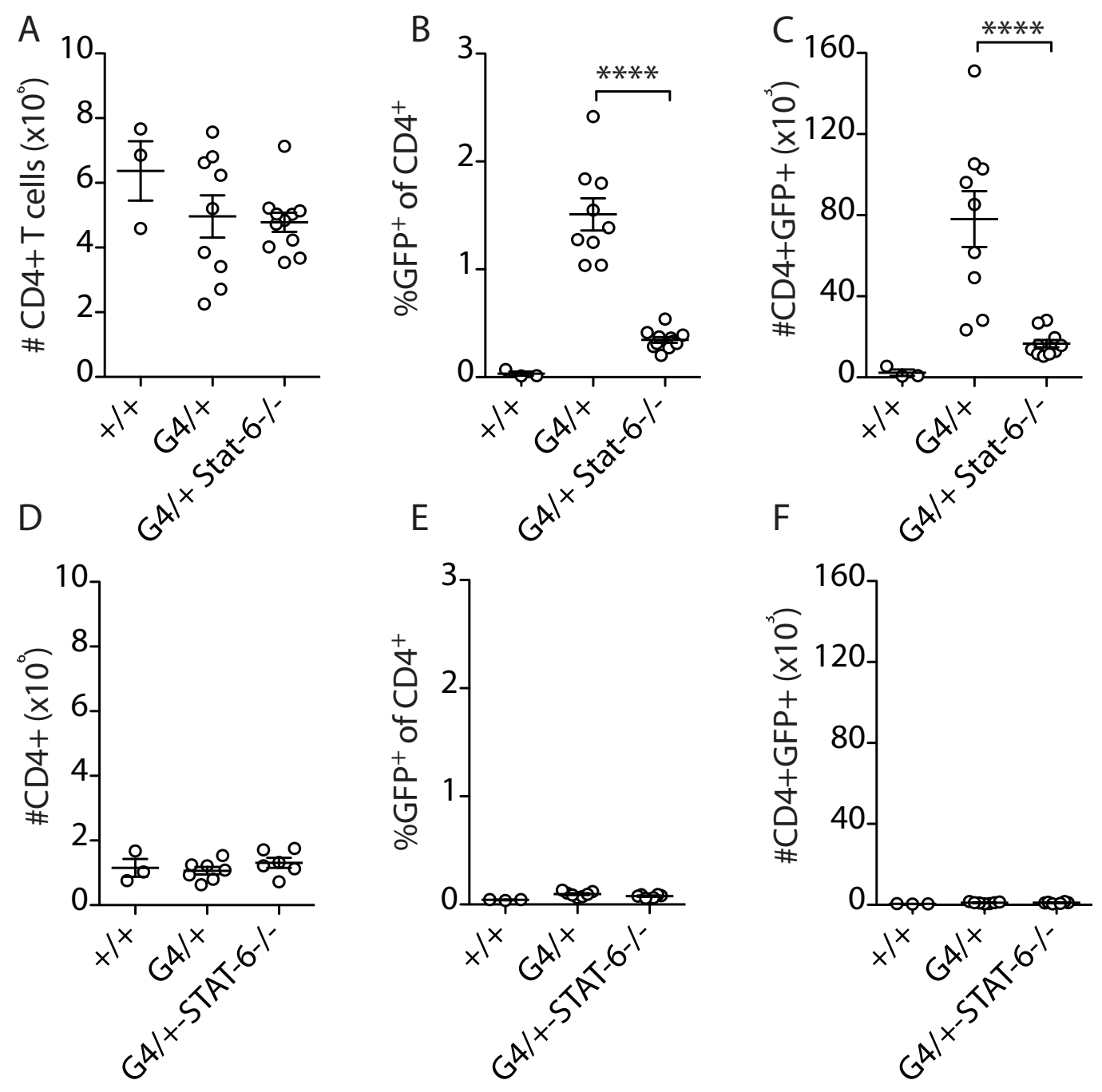

Figure 4.5: IL-4-dependent Th2 response requires STAT-6. Mice of the specified genotype were injected intradermally with $100 \mathrm{ug}$ HDM and seven days later assessed for (A) Total CD4+ T cell generation, (B) GFP expression by CD4+ T cells and $(\mathrm{C})$ the total number of Th2 cells generated. In a separate experiment naive mice of the specified genotype were euthanised and the number of (D) CD4+ $\mathrm{T}$ cells, (E) proportion of CD4+ T cells that expressed GFP and (F) total number of $\mathrm{CD} 4+\mathrm{GFP}+\mathrm{T}$ cells were examined. Data points represent individual mice and lines represent group mean \pm SEM. Data in $(A-C)$ and $(D-F)$ are from separate single experiments. Data were compared using Student's $t$-test; $* * * * \mathrm{p} \leq 0.0001$. 


\subsubsection{Examining the influence of IL-4 on Th2-dependent im- mune responses}

\section{Investigating the function of Th2 cells in B cell switch re- sponses in vivo.}

We observed reduced Th2 cell generation in IL-4-deficient mice relative to IL-4heterozygous mice. To more fully understand the effect of IL- 4 on Th2 cell development, we were next interested to determine whether genetic deletion of one IL-4 allele, in IL-4-heterozygous mice, affected Th2 cell development relative to wild-type mice. However, with no current access to an IL-4 reporter mouse with two intact IL-4 alleles $(\mathrm{IL}-4+/+)$, we cannot directly examine Th2 cell development directly using our reporter systems. Rather, we sought to investigate the influence that the loss of one IL-4 allele provides to the Th2 response by examining known IL-4-dependent responses in allergic disease contexts. The B cell class-switch response to IgG1 is an IL-4 dependent process[289], and intestinal anaphylaxis is an IL-4-dependent model of egg allergy[295]; therefore, we next sought to determine if IL-4-heterozygosity impairs either of these two processes. The experiments in this section of the chapter investigated how the absence of one IL-4 allele affects Th2 cell function. These experiments also sought to clarify the phenotype and function of Th2 cells in the pTh2 assay.

\section{Assessing the phenotype of Th2 CD4+ T cells induced by intradermal immunization}

Th2 cells have been shown to function in different capacities in lymph nodes and tissues[275, 317]. TFH cells support IgG1 antibody class-switch recombination[275], however TFH function has not yet been evaluated in the pTh2 assay. We began this set of investigations by examining whether pTh2 cells had the same phenotype as the B cell-helper TFH cell. We examined Th2 cells for expression of molecules associated with TFH cell function (CXCR5, PD-1 and ICOS[337, 338, 368, 409-411]) and for activation molecules previously observed on $N$. brasiliensis induced mediastinal 
lymph node Th2 cells (CD44, CD62L, and CD69)[1]. Our objective was to determine whether auricular lymph node Th2 cells were likely to support B cell class-switch responses.

Initially, we immunized G4/+ mice with HDM and examined expression of the early $\mathrm{T}$ cell activation molecule, CD69, three days later. In a separate group of mice, we examined CD44 and CD62L expression seven days after HDM immunization. Lastly, we determined levels of molecules expressed by TFH cells, CXCR5, PD-1 and ICOS seven days after injection of 600 dead $i$ L3 N. brasiliensis larvae. As established previously [1], Th2 cells upregulated CD44, decreased expression of CD62L and had variable, but generally elevated CD69 expression (Figure 4.6A and 4.6B). Consistent with previous reports[275, 362, 364], we observed that PD-1 and ICOS were expressed by almost all Th2 cells, and the Median fluorescence intensity of CXCR5 on the total Th2 cell population was increased over total CD4+ T cells (Figure 4.6C).

We next examined the development of $\mathrm{TFH}$ cells. In this experiment, mice were immunized intradermally with $600 i$ L3 N. brasiliensis larvae. Mock-challenged controls received $30 \mu \mathrm{L}$ PBS. Fourteen days later, we examined draining lymph node CD4+B220- T cells for the expression of CXCR5 and PD-1. When we examined wild-type mice after intradermal immunization, we observed TFH cell development in immunized, but not mock-immunized lymph nodes (Figure 4.7A and B). We also observed that TFH cells developed in G4/+ mice (Figure 4.7A and 4.7B) and were substantially enriched for IL-4 producing Th2 cells (Figure 4.7C). In all experiments, between 10 and 60 percent of GFP-expressing Th2 cells in G4/+ mice induced by intradermal immunization expressed PD-1 and CXCR5 and fell in the TFH cell gate ([275, 337]; Figure 4.7D). While the difference between TFH frequency between G4/+ and $+/+$ mice was not statistically significant (by Mann-Whitney U-test) this trend was reproducible in multiple experiments. This may relate to the role of IL- 4 in the expansion or maintenance of the Th2 population (covered earlier in this chapter and alluded to in [362]), or could also be a result of a decrease in IL-4-dependent B cell responses that contribute to TFH cell selection, as B cells have been shown to support TFH cell expansion in some studies[364]. Irrespective of the influence of IL-4 on the TFH population expansion, these results demonstrate that a substantial proportion of Th2 cells were TFH cells. 

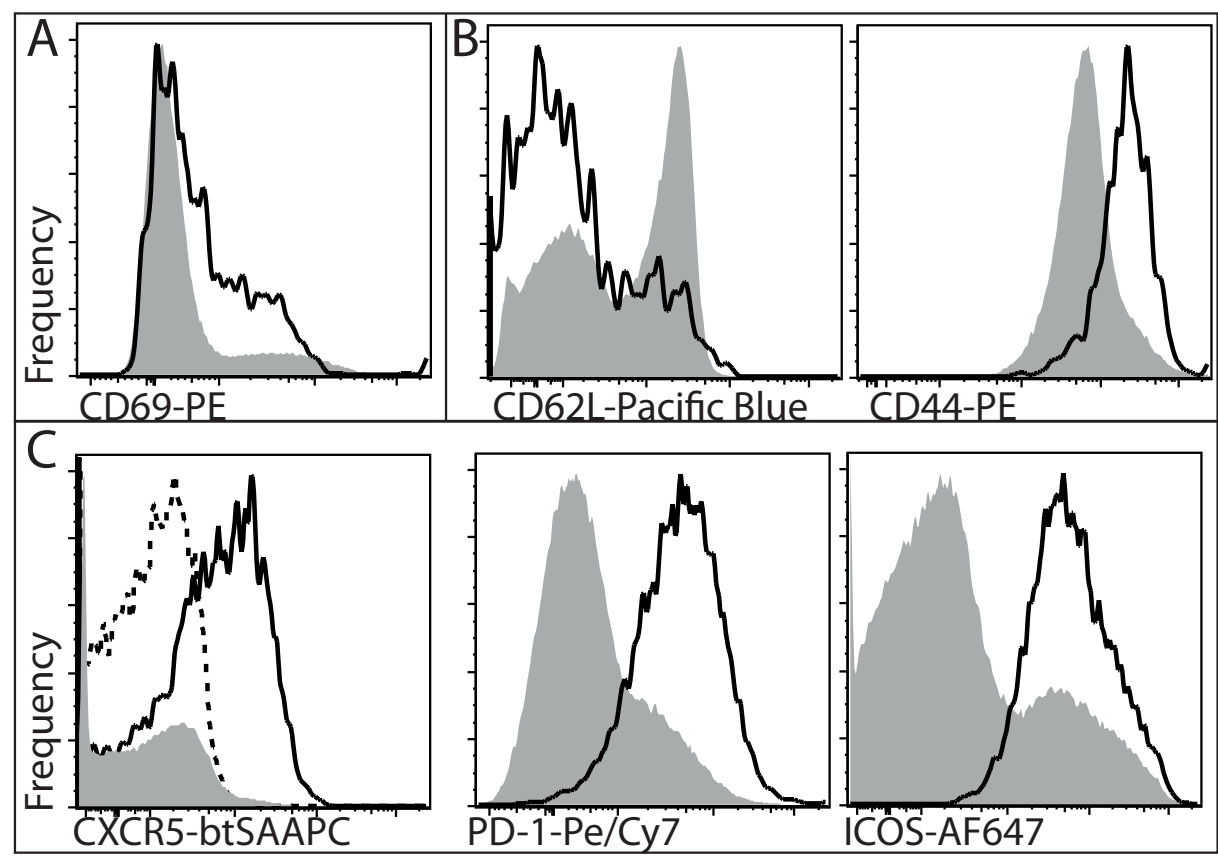

Figure 4.6: Th2 cells exhibit an activated TFH cell phenotype. Following intradermal challenge of G4/ + mice $(n=5-6)$, GFP + CD $4+$ T cells (solid black lines) and GFP- CD4+ T cells (grey histograms) were examined for surface phenotype. Isotype staining on $\mathrm{GFP}+\mathrm{CD} 4+\mathrm{T}$ cells for CXCR5 is represented by the dashed black line. Molecules associated with (A) early $\mathrm{T}$ cell activation, (B) effector $\mathrm{T}$ cell activation, and (C) TFH cells were examined. A single representative plot is shown from (A) one of two experiments done three days after HDM injection, (B) one experiment seven days after HDM injection and $(\mathrm{C})$ three or more experiments seven days after dead $i$ L3 N. brasiliensis larvae injection. Each experiment included 5-6 G4/+ mice. 

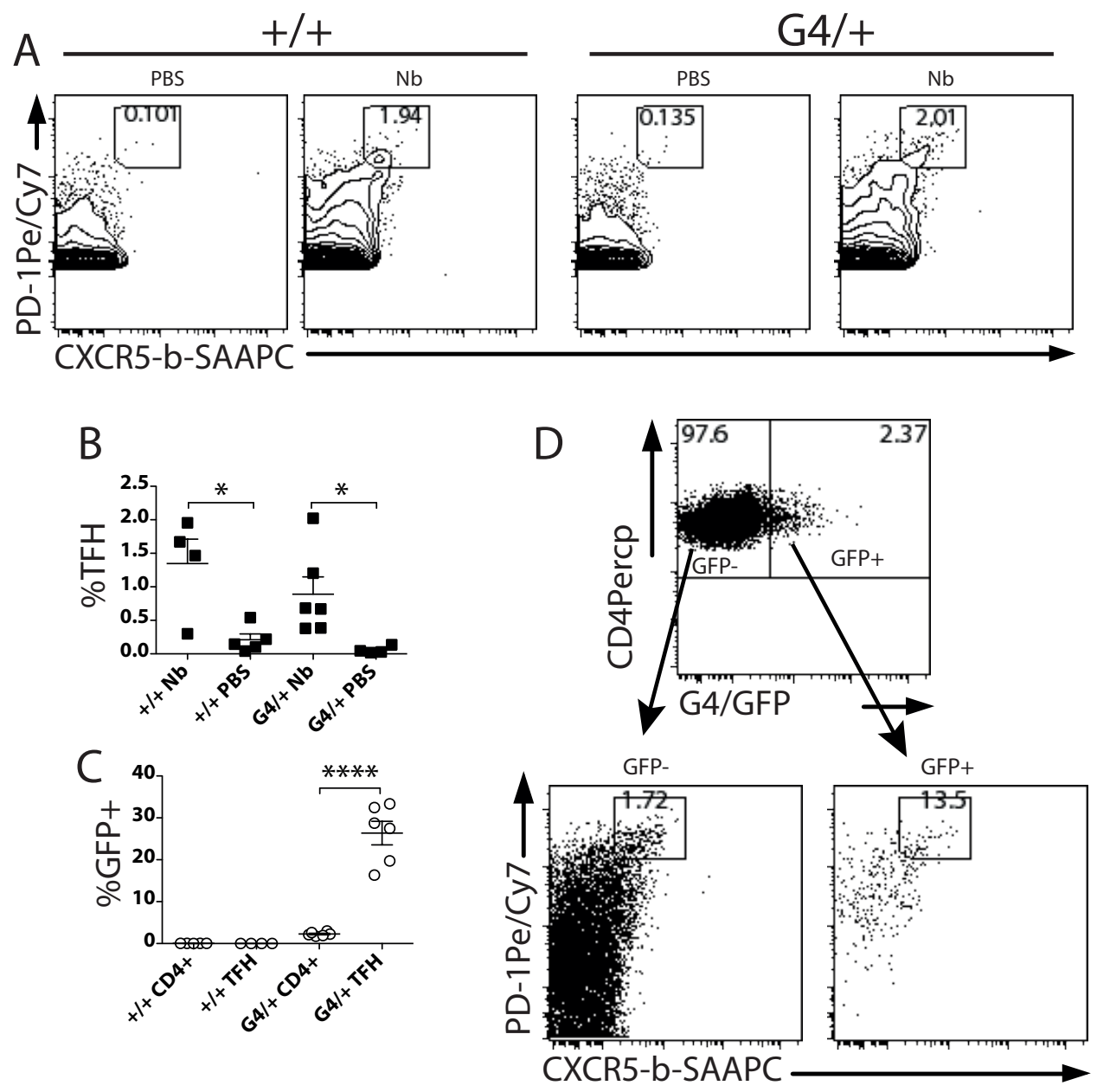

Figure 4.7: IL-4 production is common in TFH cells. Mice were intradermally challenged with 600 dead $i$ L3 $N$. brasiliensis larvae (Nb) or PBS and assessed for TFH cell development. (A) Representative plots of CXCR5 and PD-1 expression in live CD4+B220- cells. (B) Shows the proportion of CD4+ cells that fell in the canonical TFH cell gate. (C) Shows the proportion of CD4+ T cells and TFH cells that expressed GFP. (D) Shows representative CXCR5 and PD-1 expression in GFP+ and GFP-CD4+ T cells. Data shown are from one of three similar experiments. Data were compared using Student's $t$-test; ${ }^{*} \mathrm{p}<0.05,{ }^{*} * * * \mathrm{p}<0.0001$. 


\section{Investigating biallelism as a specific feature of Th2 TFH cells}

Reinhardt et al.[275] took advantage of reporter mice with one KN2 allele and the IL-4-sufficient GFP-tagged 4get mRNA reporter on the other, and observed that hCD2+4get + cells were enriched in the TFH population. In the KN2/4get mouse, transcriptional access to the IL- 4 gene is reported by 4 get expression and IL-4 production occurs predominantly in 4get+ cells co-expressing hCD2[352, 373]. As the B cell switch-inducing Th2 TFH cells were recorded as hCD2+/4get+ TFH cells, this also means that the doublet population expressed, at least at the transcriptional level, both alleles of IL-4 simultaneously. This led us to consider the possibility that biallelism is a unique property of TFH cells, and the small population of cells we observed expressing both IL-4 alleles in KN2/G4 mice (Figure 4.4) were exclusively TFH cells.

To evaluate whether biallelic cells were specifically TFH cells, we intradermally immunized KN2/G4 and +/+ mice with $100 \mu \mathrm{g}$ HDM extract. Ten days later we examined the expression of CXCR5 and PD-1 in draining lymph node CD4 $+\mathrm{T}$ cells. When we examined TFH cell development in $\mathrm{KN} 2 / \mathrm{G} 4$ mice, we found that all Th2 cell populations (hCD2+GFP-, hCD2+GFP+ and hCD2-GFP+) contained TFH (Figure $4.8 \mathrm{~B})$. Further, we also observed that hCD2 expression, rather than biallelism was the strongest predictor of TFH cell status (Figure 4.8B). Therefore, TFH cells were not exclusively biallelic. The difference in TFH frequency in hCD2+ and GFP+ cells may also indicate that the G4 and KN2 reporters demarcate T cells with separate functions, at different stages of development, or with different fates. Future experiments will be required to determine the relevance of the differential population frequencies. 

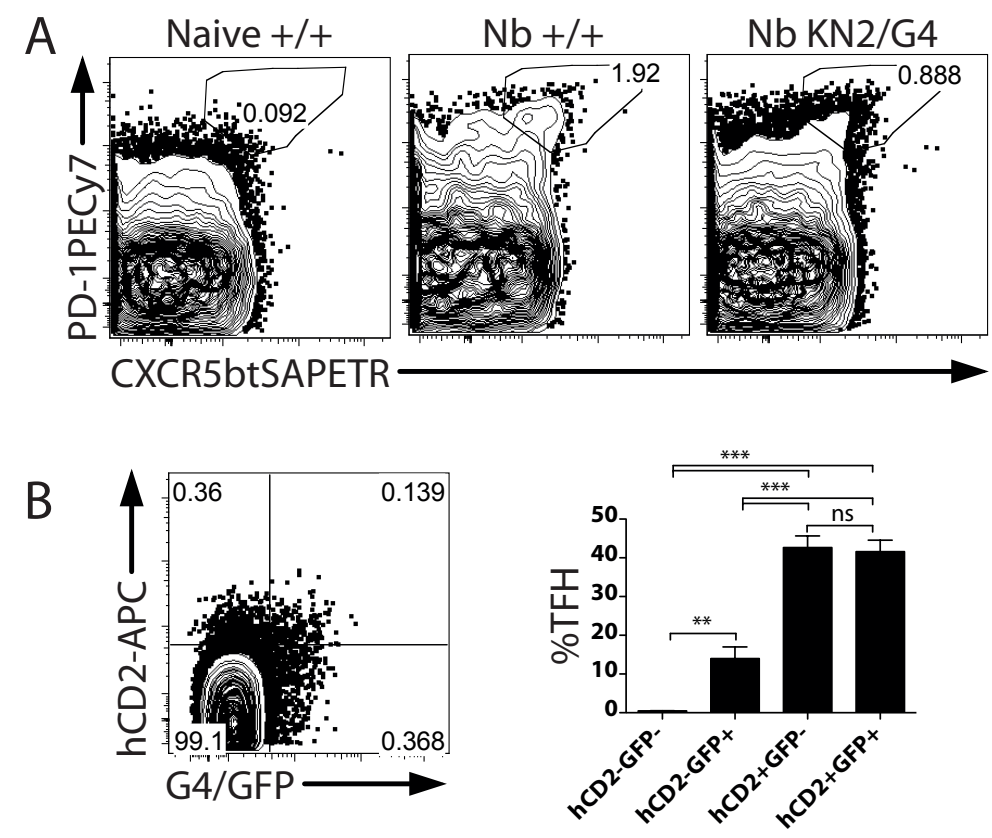

Figure 4.8: IL-4 can derive from one or both alleles in TFH cells. (A) Lymph nodes from mice of the indicated genotype were examined for CXCR5+PD-1hi TFH cells after immunization with 600 dead $i$ L3 $N$. brasiliensis larvae. (B) Ten days after immunization with $100 \mathrm{ug} \mathrm{HDM}$, CD4+ T cells from KN2/G4 mice expressing neither hCD2 nor GFP (hCD2-GFP-), only GFP (hCD2-GFP+), only hCD2 (hCD2+GFP-) or both hCD2 and GFP (hCD2+GFP+) were assessed for the proportion of cells in that quadrant that fell in the CXCR5+PD-1Hi TFH gate. A representative plot is shown on the left, and coordinates were set to $<0.05$ percent in each quadrant in CD4 $+\mathrm{T}$ cells in similarly immunized $+/+$ mice. Bars in the graph shown in $(\mathrm{B})$ represent mean \pm SEM of $n=12$ mice combined from three experiments. 


\section{The GL-7+ germinal centre TFH population includes a signif- icant number of IL-4-producing cells}

We reasoned that if the pTh2 response initiated IL-4-dependent B cell responses, we would observe germinal centre formation in immunized lymph nodes. GL-7 is expressed by both germinal centre B cells[338] and germinal centre TFH cells that contain high levels of IL-4 mRNA[366]. We next used GL-7 expression to determine if GC B and Th2 TFH cells are present in draining auricular lymph nodes.

In these experiments, we intradermally immunized G4/ + and $+/+$ mice with $100 \mu \mathrm{g}$ HDM antigen. Seven days later, we harvested draining lymph nodes, and non-draining contralateral lymph nodes and examined GL-7 expression on B cells and CD4+ TFH cells. We found that the proportion of GL-7-expressing B cells in the lymph node was increased in draining lymph nodes (Figure 4.9B). Therefore, GL-7-expressing B cells developed in response to immunization. Similarly, GL-7 expression by CD4+ T cells was predominantly by PD-1-expressing cells (Figure 4.9C), and we observed that a large proportion of TFH cells expressed GL-7 (Figure 4.9D and 4.9E). We observed only minor differences in the proportion of GL-7+ and GL-7- TFH cells that expressed GFP (Figure 4.9F). This suggests that GL-7- TFH have also been induced to produce IL-4. These findings indicate that auricular pTh2 cells have the potential to support IL-4-dependent B cell isotype class-switching. 

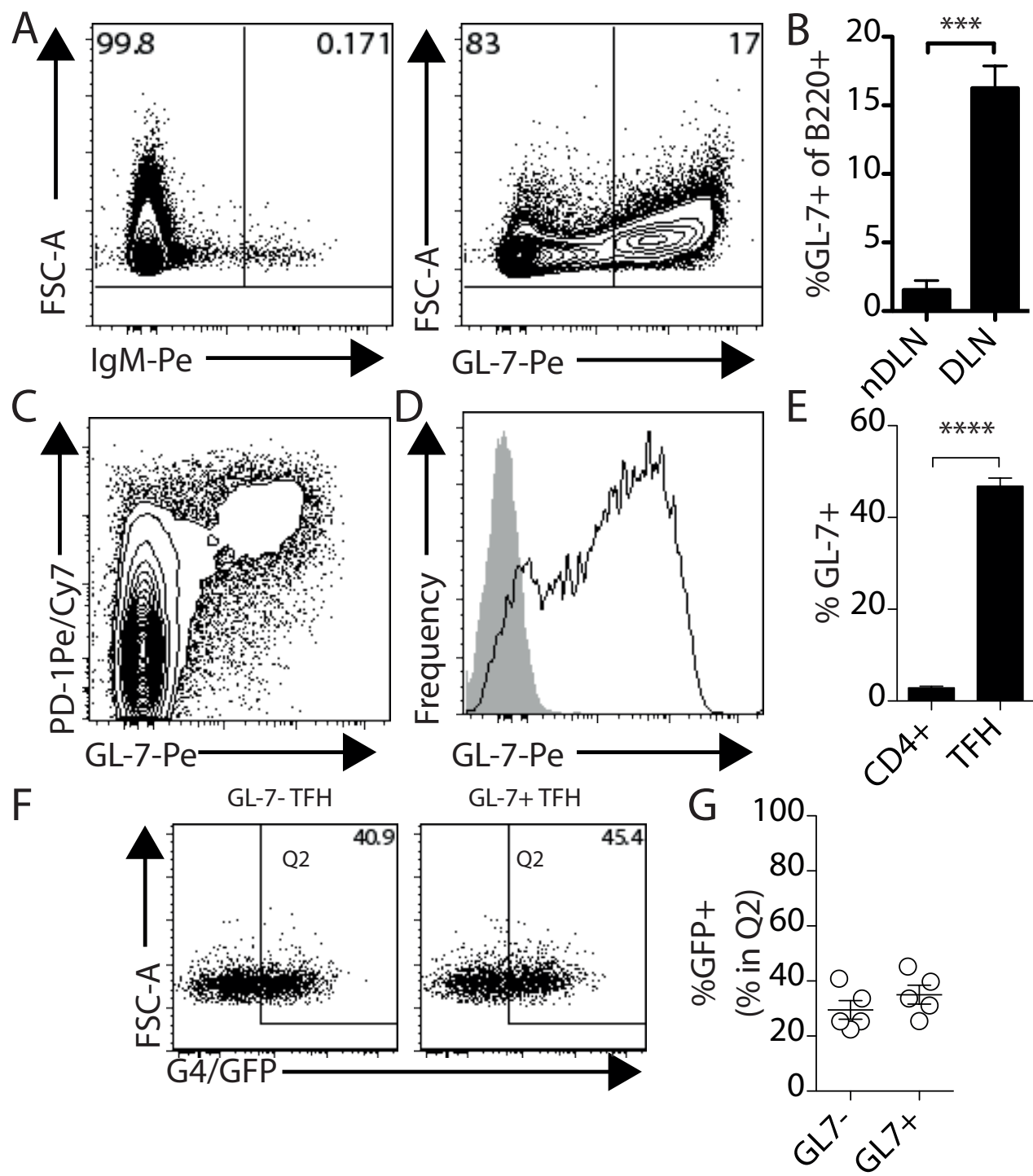

Figure 4.9: IL-4 is produced by GL-7+ (germinal centre) TFH cells. (A) Seven days after intradermal immunization with $100 \mu \mathrm{g}$ HDM, B cells were stained with an IgM isotype (left) or anti-GL-7 antibody (right) to assess germinal centre B cell development. The gates shown on the plots define GL-7+ and GL-7- cells analyzed in (B) and (E-G). (B) The proportion of live B220+ cells expressing GL7 was compared in non-draining contralateral (nDLN) and draining (DLN) lymph nodes. (C) CD4+ T cells were examined for PD-1 and GL-7 expression by flow cytometry. (D) GL-7 expression was examined in TFH cells (black line; gated as shown in Figure 4.8) and compared to isotype stained TFH (grey histogram). (E) The proportion of CD4+ T cells and TFH cells expressing GL-7 was examined. (F) Representative flow plots of GFP expression in GL-7- and GL-7+ TFH cells(left), (G) in which the proportion of cells expressing GFP (percent in Q2) were compared. Data points represent individual mice; bars and lines represent group mean \pm SEM. Data are shown from one ( $\mathrm{n}=3-5$ mice) of four similar experiments, two using HDM and two using dead $i$ L3 $N$. brasiliensis larvae as the immunogen. Data were compared using Student's $t$-test; ${ }^{* * *} \mathrm{p}<0.001,{ }^{* * * *} \mathrm{p}<0.0001$. 


\section{Intradermal immunization induces IgG1 isotype class switch- ing}

Germinal centre Th2 TFH cells developed in mice immunized with HDM in the absence of exogenous adjuvant. Our next objective was to examine whether IgG1 isotype class-switching could be observed without adjuvant.

In these experiments, we intradermally immunized $+/+$ mice with either $100 \mu \mathrm{g}$ HDM antigen or 600 dead $i$ L3 $N$. brasiliensis larvae. Sixteen days later, we examined IgG1 and $\mathrm{IgD}$ expression on B220+ B cells in draining and contralateral non-draining lymph nodes. We observed a distinct population of IgG1+IgD-B220+ cells in draining, but not non-draining lymph nodes of both immunized groups (Figure 4.10A - C). When we compared the HDM and parasite antigens, we observed that twice as many IgG1+IgDB220+ cells developed in response to the parasite antigens (Figure 4.10C). Overall, these data suggest that parasite antigens and HDM both induce IgG1 class switching in the absence of exogenous adjuvant.

In a separate experiment, we examined the kinetics of IgG1+IgD-B220+ cell development. After performing optimisation experiments with a new batch of HDM antigen (data not shown), in draining lymph nodes of mice immunized with $200 \mu \mathrm{g}$ HDM, the number of $\operatorname{IgG1}+\operatorname{IgD}$-B220+ cells increased with time and became significantly elevated on day 16 (Figure 4.10D). These data indicate that IgG1+IgD- B cells develop with the kinetics expected of an affinity matured, CD4 $+\mathrm{T}$ cell-dependent germinal centre response.

We were also interested to determine if we could observe systemic antibody production following intradermal immunization. In parallel with the assays described (Figure 4.10), we bled mice and froze serum samples prior to, seven and sixteen days after intradermal immunization. We then tested for HDM-specific IgG1 antibody in HDMand parasite antigen-immunized mice. We detected HDM-specific IgG1 in day 16 sera, but not in day 0 or 7 sera (Figure 4.11A). We did not detect HDM-specific IgG1 in sera from dead $i$ L3 N. brasiliensis-immunized mice (Figure 4.11B). These data suggest that the intradermally-induced auricular lymph node IgG1 response is antigen-specific. 

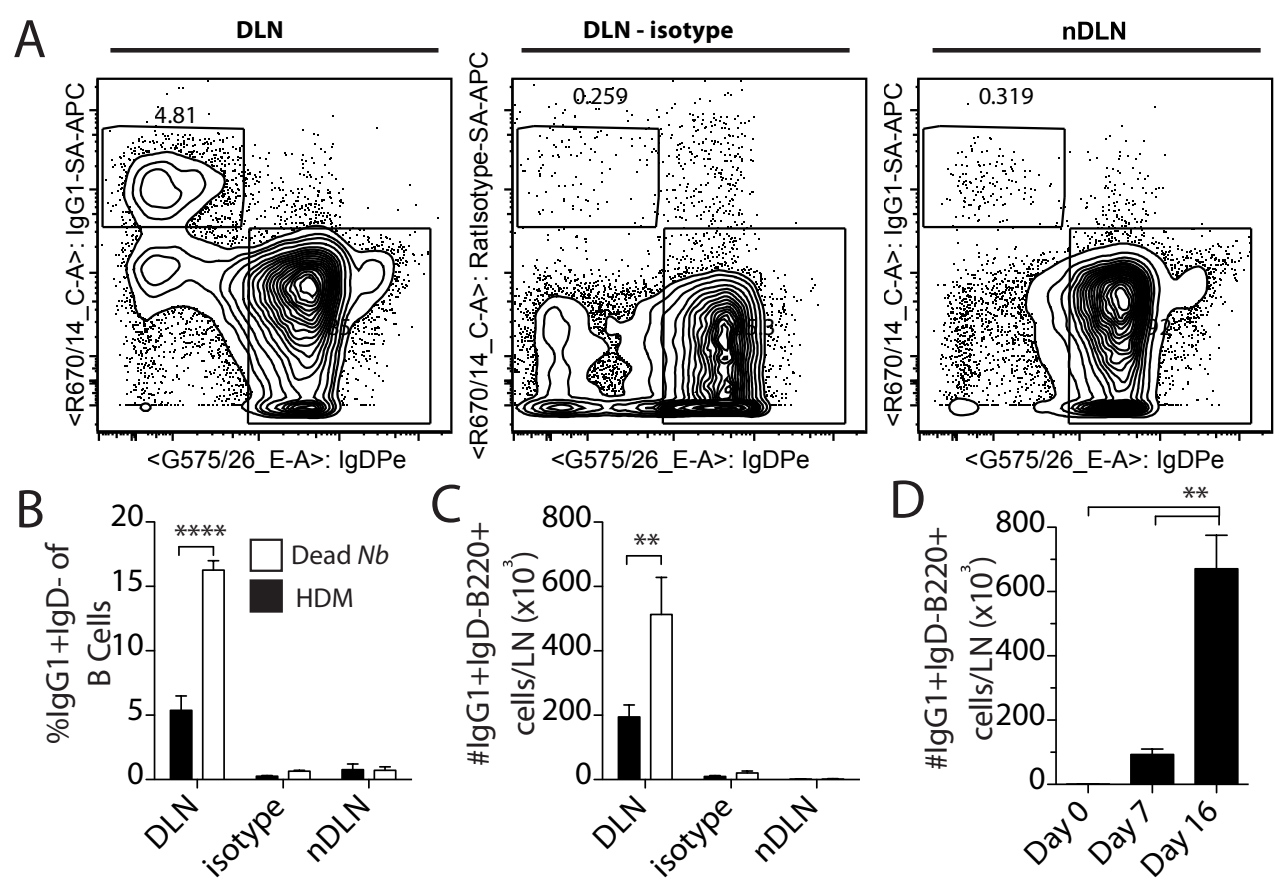

Figure 4.10: TFH cell function is reported by IgG1 + B cell development. (A) Sixteen days after intradermal immunization with 100 ug HDM live auricular lymph node B220+ cells were examined for IgD and IgG1 expression by flow cytometry in draining (DLN) and non-draining (nDLN) lymph nodes. The proportion (B) and total number $(\mathrm{C})$ of B220+ cells that stained for surface IgG1 and not IgD was compared in mice immunized with 100 ug HDM (black bars) or 600 dead $i$ L3 N. brasiliensis larvae (white bars) ( $\mathrm{n}=3$ /group). (D) The kinetic generation of IgG1+IgD-B220+ cells was measured in mice immunized with $200 \mathrm{ug}$ HDM antigen in a separate experiment. Data shown in (A-C) are from one of three similar experiments. The data shown in (D) was performed and analysed in a single experiment. Data were compared by One-Way ANOVA with Tukey's post-test; ${ }^{* *} \mathrm{p}<0.01,{ }^{* * * *} \mathrm{p}<0.0001$. 

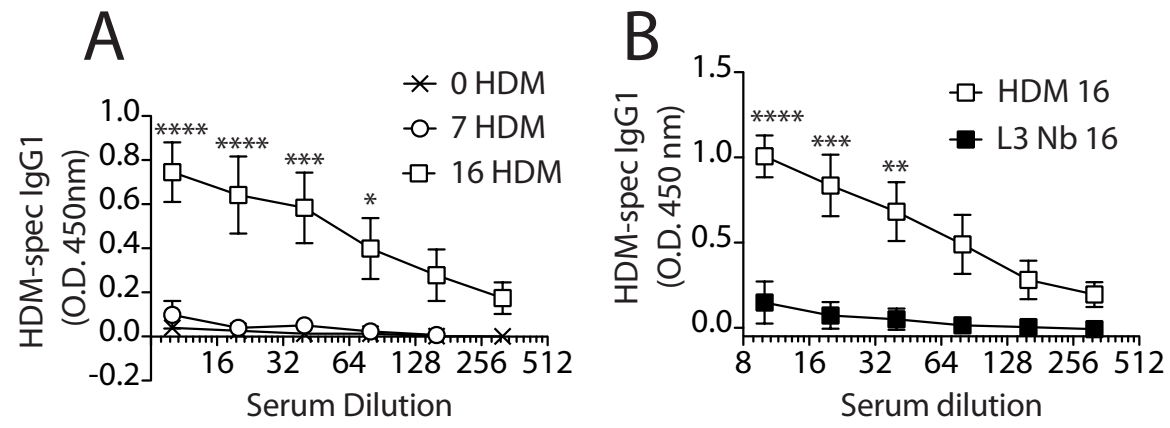

Figure 4.11: IgG1 class-switch B cell response is antigen-specific. (A) Prior to (crosses) and seven (circles) and sixteen (squares) days after intradermal injection of $100 \mathrm{ug}$ HDM, mouse serum was analysed by ELISA for HDM-specific IgG1. (B) Sixteen days after intradermal challenge with $100 \mathrm{ug}$ HDM (white squares) or 600 dead $i$ L3 $N$. brasiliensis larvae (black squares), mouse serum was analysed by ELISA for HDM-specific IgG1. Data points show mean \pm SEM of groups of $(n=3)$ mice from one of three similar experiments. Data were analysed using (A) Two-way repeated measures ANOVA with Bonferroni's post-test versus day 0 and day 7 sera, or (B) Two-way ANOVA with Bonferroni's post-test versus L3 Nb; ${ }^{*} \mathrm{p}<0.05,{ }^{* *} \mathrm{p}<0.01,{ }^{* * *} \mathrm{p}<0.001$, $* * * * \mathrm{p}<0.0001$. 


\subsubsection{The influence of a single IL-4 allele on primary, adjuvant- free IgG1-class switch recombination}

Parasite-induced IgG1 class-switching is reduced by approximately 80 percent in IL-4 deficient mice[289] and also requires STAT-6[330], however, signals other than IL4[274, 287, 288], specifically CD40:CD40 ligand (L) interaction[291, 292, 412-414], contribute to isotype class-switching[274, 415]. Further, specific types of immunization induce STAT-6- and IL-4-independent IgG1 production[274, 329], and in special circumstances, even IgE can be generated in the absence of IL- 4 or CD4+ T cell help[416-418]. Therefore, it was possible that we were observing IL-4-independent IgG1 class-switching in the auricular lymph node. We next designed experiments to assess the involvement of IL-4 in the B cell IgG1 class-switch response. To do this we used mice deficient in STAT-6 or IL-4 and examined the influence that the loss of a single IL-4 allele has on the B cell class-switch response.

In these experiments, we immunized +/+, STAT-6-/-, G4/+ or G4/G4 mice with 100 $\mu \mathrm{g}$ HDM. Fourteen days later, we tested draining and non-draining lymph nodes for IgG1+ B cells. Further, because MHC-II is upregulated on activated B cells in an IL-4- and STAT-6-dependent manner[329, 330, 419], we also examined the expression of MHC-II on the B cells.

As expected, STAT-6-deficient mice exhibited impaired IgG1 switching (Figure 4.12A). Correspondingly, B cells in these draining lymph nodes of STAT-6-deficient mice did not respond to IL-4, because expression of MHC-II was lower than observed in draining lymph nodes of IL-4-sufficient mice (Figure 4.12B). When we examined Th2 cell development in G4/G4-STAT-6-/- mice, we observed a minor elevation in the proportion of $\mathrm{GFP}+\mathrm{CD} 4+\mathrm{T}$ cells present in the draining, but not the non-draining lymph node (Figure $4.12 \mathrm{C}$ ). This indicates that Th2 cells developed, but were not able to support IgG1 switching in STAT-6-deficient conditions. While the MedFI of I-A/I-E on the B cells in the non-draining $+/+$ lymph node was higher than others, comparison to naive lymph nodes (which were not included) would be required to determine whether this reflects dissemination of the $\mathrm{B}$ cell response or not. Therefore, while Th2 cells developed without either IL-4 or STAT-6, IgG1-switched B cell hyperplasia induced by intradermal immunization was STAT-6 dependent. 

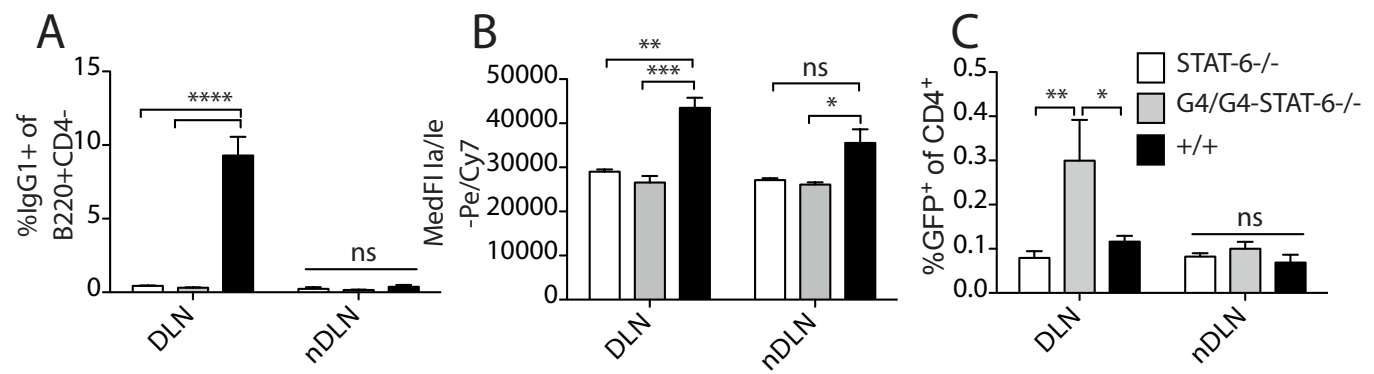

Figure 4.12: IgG1 class-switch B cell response requires STAT-6. Mice were intradermally immunized with $100 \mathrm{ug}$ HDM and fourteen days later draining (black bars/symbols) and non-draining (white bars) lymph nodes were excised and analysed by flow cytometry for IgG1 expression by B220+ cells and GFP expression by CD4+ cells. (A) The proportion of B220+ cells expressing IgG1 was examined. (B) the expression of MHC-II (I-A/I-E) on the B220+ population was compared in mice of different genotypes. (C) The proportion of CD4+ T cells that expressed GFP was evaluated in the different groups. Data are shown from a single experiment $(\mathrm{n}=3-5$ mice/group) and were analysed by Two-way repeated measures ANOVA with Bonferroni's post-test. ${ }^{*} \mathrm{p}<0.05,{ }^{* *} \mathrm{p}<0.01,{ }^{* * *} \mathrm{p}<0.001,{ }^{* * * *} \mathrm{p}<0.0001$. 
While STAT-6 was required for the adjuvant-free IgG1 class-switch response, low levels of IgG1 and IgE have been observed in IL-4- and IL-4R $\alpha$-deficient mice[418]. To examine the influence of each IL-4 allele on IgG1 class-switching, we examined class-switching in IL-4-deficient G4/G4 and IL-4-heterozygous G4/+ mice. Fourteen days later, we examined mice for IgG1+ B cells, the expression of MHC-II, and Th2 cell development.

We observed that Th2 cells developed in G4/G4 and G4/G4-STAT-6-/- mice (Figure 4.13C). This indicates that Th2 cells were able to develop in IL-4-deficient and IL-4 heterozygous mice. However, we observed that $\operatorname{IgG} 1+\operatorname{IgD}-\mathrm{B}$ cells did not develop in G4/G4 mice (Figure 4.13A). Additionally, we observed that MHC-II expression was lower in G4/G4 and G4/G4-STAT-6-/- IgG1+ cells than in IL-4 sufficient G4/+ and $+/+$ draining lymph node IgG1+ B cells (Figure 4.13E). Therefore while Th2 cells developed in the absence of IL-4 (Figure 4.13) IL-4 was required for adjuvant-free B cell IgG1 class-switch recombination. Similarly in G4/+ mice, an increase in Th2 cell number was observed in draining lymph nodes but not non-draining lymph nodes (Figure 4.13C). When we examined the B cell response, we observed that a single IL-4sufficient allele was sufficient to permit B cell MHC-II upregulation (Figure 4.13B), but was not sufficient to permit normal IgG1 induction, because these mice demonstrated a two-fold reduction in proportional switching relative to $+/+$ mice (Figure 4.13A). Thus, a $50 \%$ decrease in the number of functional IL-4-producing alleles impaired the IL-4-dependent B cell class-switch response. Taken together, these data indicate that the loss of a single IL-4 allele in mice impairs either Th2 cell development or TFH cell function relative to wild-type mice. 

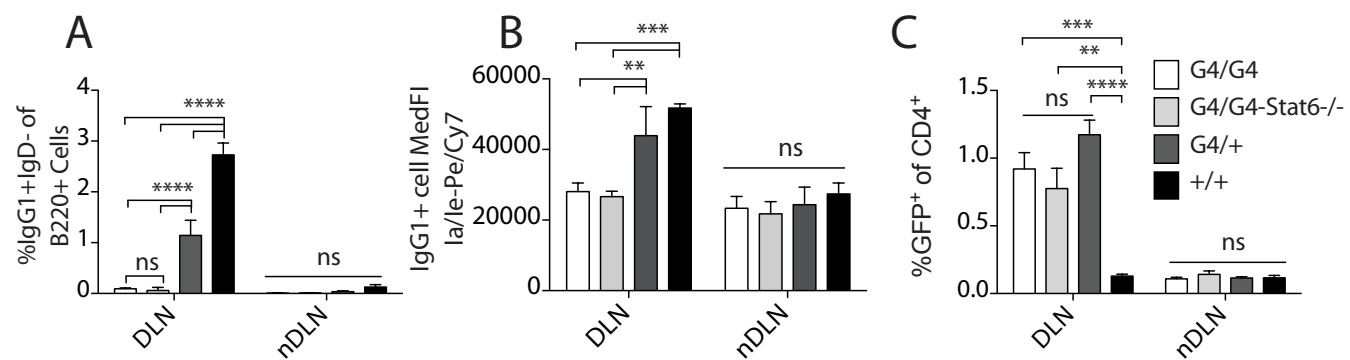

Figure 4.13: The IgG1 class-switch response is IL-4 allele dose-dependent. Fourteen days after intradermal injection of $0.2 \mathrm{mg}$ HDM antigen, draining (DLN) and non-draining (nDLN) auricular lymph node B cells were examined for IgG1 and $\mathrm{IgD}$ expression (A) and CD4+ cells were examined for GFP expression (C). The expression of MHC-II (I-A/I-E) on IgG1+B220+ LN cells was compared (B). Data are shown from one of two similar experiments $(\mathrm{n}=2-3$ mice/group). (A-C) Data were analysed by Two-way repeated measures ANOVA with Bonferroni's post-test; ${ }^{* *} \mathrm{p}<0.01,{ }^{* * *} \mathrm{p}<0.001,{ }^{* * * *} \mathrm{p}<0.0001$. 


\subsubsection{The influence of IL-4 heterozygosity on the intestinal allergic response}

As a final investigation of the effect of IL-4 heterozygosity on Th2-type allergic immune responses, we examined the development of oral allergen-induced intestinal anaphylaxis in IL-4-heterozygous mice.

In these experiments, mice were intraperitoneally immunized with $50 \mu \mathrm{g}$ OVA in the presence of $1 \mathrm{mg}$ alum adjuvant, twice, two weeks apart. Mice were then bled, and examined for serum IgE levels. In one experiment, mice received two further fortnightly intraperitoneal immunizations. Following this sensitization phase, mice were orally gavaged with $50 \mathrm{mg}$ OVA, and monitored for diarrhoea development. Mice were bled after the $9^{\text {th }}$ challenge to measure haematocrit, and were bled after the $10^{\text {th }}$ challenge to examine murine mast cell protease-1 (mMCP-1) and antibody titres in the serum. In these comparisons the phenotype of $\mathrm{G} 4 /+$ and $\mathrm{KN} 2 /+$ mice were essentially the same, so I will discuss these mice collectively as $+/-$ mice. In figures the response of mice from each genotype are shown.

When we examined IgE generation in mice immunized twice with OVA and alum, we observed $90 \%$ less IgE in +/- mice than in wild-type $+/+$ mice (Figure 4.14A). When we examined IgE levels in mice that had received a total of four fortnightly i.p. sensitizations, and then ten oral OVA challenges, we still observed a 90\% decrease in serum $\operatorname{IgE}$ levels (Figure 4.15A), and also lower levels of OVA-specific IgE in +/compared with $+/+$ mice (Figure 4.15B). These data indicate that allergen-specific $\mathrm{IgE}$ production is impaired in IL-4-heterozygous mice.

When we challenged mice orally with OVA, only $+/+$ mice exhibited diarrhoea (Figure 4.16A). Blood haematocrit was higher in diarrhoetic $+/+$ mice than $+/$ - mice (Figure 4.16B). These data demonstrate that $+/$ - mice did not develop an intestinal allergic response. When we examined mast cell activity in these mice, we observed that mMCP-1 titres were lower, and jejunal mast cells were less frequent in OVA challenged $+/$ - mice than $+/+$ mice (Figure 4.16C and 4.16D). While we did not examined basal mMCP-1 levels in IL-4 +/- mice and will require confirmation, basal levels in wildtype mice are usually approximately $20-40 \mathrm{ng} / \mathrm{mL}$, indicating some increase in intestinal 
mast cell activity may have occurred in the orally challenged $+/-$ mice. These data indicate that both IL-4 alleles are required for normal oral allergen-induced mast cell activity.

Taken together, these data suggest that both IL-4 alleles contribute to IgE development. Mice deficient in a single IL-4 allele exhibited deficient IgE generation, impaired intestinal mast cell activity and were protected from allergic diarrhoea. These data suggest that both IL-4 alleles are required for normal allergen-specific Th2 immune responses. 


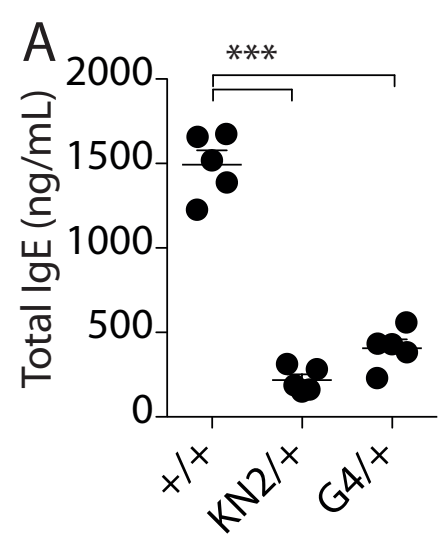

Figure 4.14: IgE production is impaired in IL-4 heterozygous mice. (A) Mice were sensitized intraperitoneally with $50 \mu \mathrm{g}$ OVA plus $1 \mathrm{mg}$ alum and 28 days later were bled and serum was analysed for Total IgE. Data are shown from one of four separate experiments in $+/+$ and $\mathrm{G} 4 /+$ mice $(\mathrm{n}=3-5$ mice). KN2 $/+$ mice $(\mathrm{n}=5)$ were analysed at this time-point once. Data were analysed by One-way ANOVA with Tukey's post-test; ${ }^{* * *} \mathrm{p}<0.001$. 


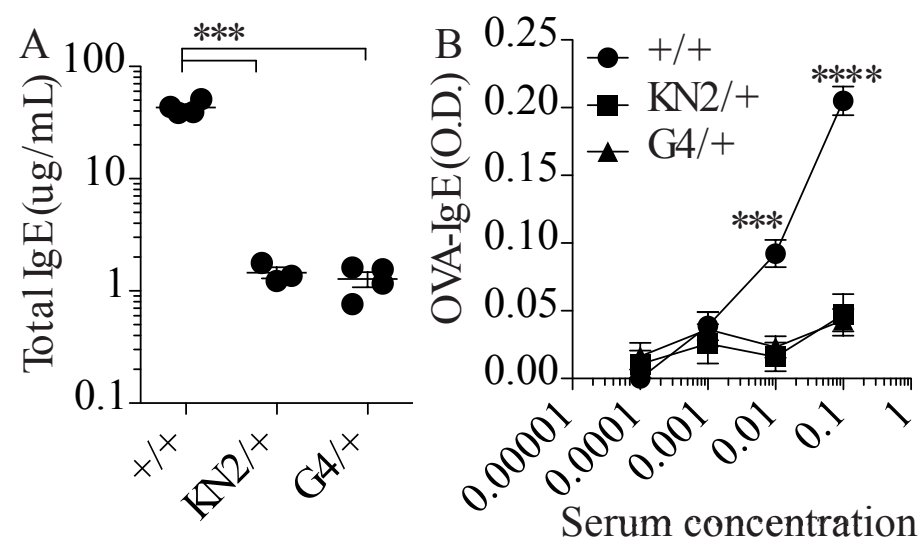

Figure 4.15: IgE production is impaired in hyper-primed, orally-challenged, IL-4 heterozygous mice. Mice were sensitized intraperitoneally four times, every two weeks with $50 \mu \mathrm{g}$ OVA and $1 \mathrm{mg}$ alum. Two weeks after the fourth i.p. prime, mice were then orally challenged with $50 \mathrm{mg}$ OVA ten times as per the standard regimen. Sixty minutes after the final challenge, mice were bled for (A) Total $\operatorname{IgE}$ and (B) OVA-specific IgE. Data points in (A) show individual mice and in (B) data points represent mean \pm SEM of these same $(n=3-5)$ mice from a single experiment. Data were analysed by (A) One-Way ANOVA with Tukey's post-test, or (B) Two-way ANOVA with Bonferroni's post-test; ${ }^{* * *} \mathrm{p}<0.001,{ }^{* * * *} \mathrm{p}<0.0001,+/+$ versus KN2/+ or $\mathrm{G} 4 /+$. 

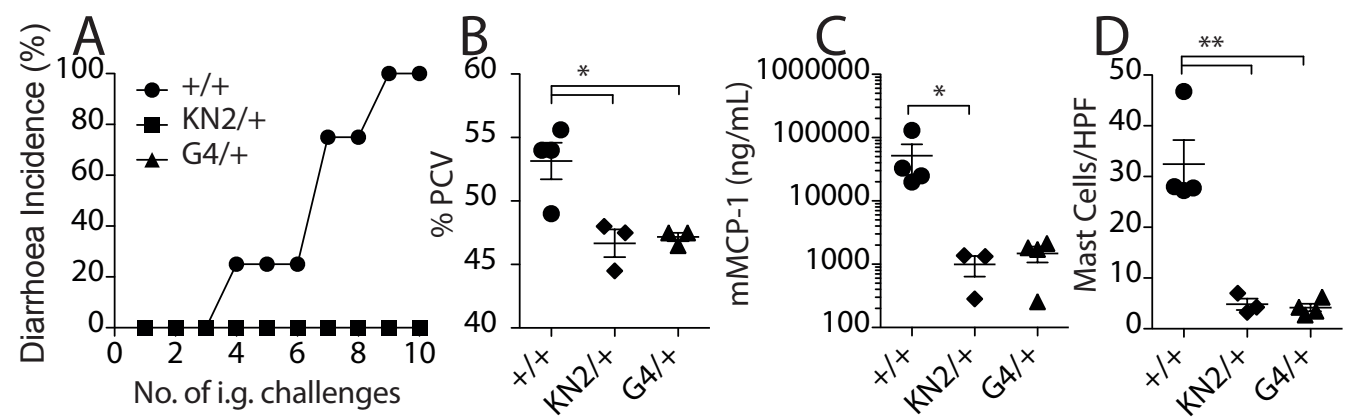

Figure 4.16: IL-4 heterozygosity prevents oral-allergen induced intestinal anaphylaxis. Mice shown in Figure 4.15 were (A) monitored for diarrhoea development following challenges and (B) haematocrit (\%PCV) 60 minutes after the ninth challenge. (C) mMCP-1 was measured from sera taken 120 minutes after the final gavage challenge. (D) Intestinal mast cell infiltration at the experimental end-point. Data are shown from a single experiment and were analysed using One-way ANOVA with Tukey's post-test; ${ }^{*} \mathrm{p}<0.05,{ }^{*} \mathrm{p}<0.01$. mMCP-1 data were log-transformed prior to analysis. In two additional experiments, $\mathrm{G} 4 /+$ mice $(\mathrm{n}=5 /$ experiment) that received two ip primes did not exhibit diarrhea with ten $50 \mathrm{mg}$ OVA challenges. 


\subsection{Discussion}

The aim of this chapter was to evaluate the role of IL-4 in Th2 cell development and function in vivo. In these studies, we employed a primary Th2 response assay to examine the involvement of IL-4 in Th2 cell development. We subsequently examined the phenotype of Th2 cells responding to intradermal allergen immunisation, and then examined their ability to support IgG1 class-switch responses. We observed significant impairments in Th2 cell development in IL-4-deficient conditions, and demonstrated that both IL-4 alleles were required for the generation of allergen-specific IgG1, IgE and for intestinal allergic responses. These studies illustrate the importance of IL-4 in the co-ordination of allergic Th2 immune responses.

\subsubsection{The role of IL-4 in Th2 cell development}

Th2 cell development was observed to occur in two phases in our experiments. We observed that similar numbers of GFP $+\mathrm{CD} 4+\mathrm{T}$ cells developed in G4/ + and KN2/G4 mice three days after adjuvant-free HDM immunization, but after this time G4/+ lymph nodes contained approximately $50 \%$ more GFP+CD4+ T cells than KN2/G4 lymph nodes (Figure 4.2). Thus, there was an IL-4-independent initiation phase, followed by an IL-4-dependent amplification phase. Similar IL-4-independent and dependent phases were observed when we compared hCD2+CD4+ T cell development in $\mathrm{KN} 2 /+$ and KN2/G4 mice (Figure 4.3). We also observed a requirement for STAT6 in Th2 cell expansion, because GFP+CD4+ T cells were fewer in G4/+-STAT-6-/mice than $\mathrm{G} 4 /+$ mice seven days after immunisation (Figure 4.5). Collectively, these data indicated that Th2 cell development was initially IL-4-independent, but was then amplified in the presence of IL-4. These data support the accumulating body of literature that indicates that Th2 cell development can be initiated in an IL-4-independent manner.

Our studies examined adjuvant-free allergen-induced Th2 cell development, but similar conclusions have been reached using adjuvant-based protein and hapten immunisation regimens. Cunningham et al. examined the requirement for IL-4 signaling using mice deficient in IL-4R $\alpha[360]$. In their studies, mice were immunised with 
haptenated fowl gamma globulin, alum and heat-killed Bordetella pertussis. To measure Th2 development, IL-4 mRNA transcript frequencies were compared in isolated draining lymph nodes of IL-4R $\alpha^{+/+}$and IL- $4 \mathrm{R} \alpha^{-/-}$mice. They observed similar increases in IL-4 mRNA transcript levels and lymph node expansion in both strains for the first three days[361]. After this time, in IL-4R $\alpha$-deficient mice, lymph nodes atrophied and $\epsilon$ - and $\gamma 1$-switch responses were diminished[361]. IL-4 mRNA transcripts were, however, still detected[361]. Thus, the induction of IL-4 production occurred normally without IL-4R $\alpha$, but IL-4-dependent B cell responses required IL4 signaling. Similarly, Reinhardt et al. examined Th2 cell development in response to haptenated-bovine serum albumin adsorbed to alum[275]. They observed that Th2 cells developed in both KN2/+ and KN2/KN2 mice, but hapten-specific IgG1 production was decreased in KN2/KN2 mice. van Panhuys et al. immunised mice with keyhole limpet hemocyanin and alum, and then gave them an airways challenge with keyhole limpet hemocyanin[1]. They observed that mediastinal Th2 cell numbers expanded similarly in G4/+, G4/G4 and G4/G4-STAT-6-/- mice, and airways eosinophilia was unimpaired, but IgE production was ablated[1]. Our studies add to these findings and demonstrate that adjuvant-free Th2 cell development also does not require IL-4. Collectively these data indicate that there is no absolute requirement for IL-4 in antigen-induced Th2 cell development, but IL-4 remains an important molecule in Th2-mediated B cell responses.

Studies have also examined the role of IL- 4 in other Th2 response models. van Panhuys, King, Noben-Trauth, Mohrs et al.[1, 362, 363, 420] examined the development of the Th2 cell phenotype in parasite-infected mice. Seven days after $H$. polygyrus bakeri infection, Th2 cell numbers were similar in IL-4Ra-/-4get and IL-4Ra+/+4get reporter mice, but by day 14, IL-4Ra-/- lymph nodes contained fewer 4get+ cells and fewer B cells than IL-4Ra+/+ counterparts[362]. Similarly, when $N$. brasiliensis was used to infect G4/+ and G4/G4-STAT-6-/- mice, Th2 cells developed with similar kinetics during primary infection, and were even observed to expand to higher absolute frequencies following a secondary challenge[1]. T cells from $N$. brasiliensis- or Leishmania major-infected IL-4R $\alpha$-deficient mice have been shown to produce measurable amounts of IL-4 upon ex vivo restimulation[363, 420]. Th2 cell development has also been demonstrated to be unimpaired in anti- $\operatorname{IgD}$ treated mice deficient in 
IL-4, IL-4R $\alpha$ or STAT-6[1, 421]. Additionally, it has also been demonstrated that dead parasite antigens induce Th2 cell development in intradermally immunised IL-4 and STAT-6 doubly deficient mice[1]. Collectively, there are numerous studies that indicate that Th2 cell development can occur in the absence of IL-4 in vivo.

IL-4-independent and -dependent phases of Th2 cell development have been reported not only in vivo, but also in vitro. While IL-4 enhances Th2 cell committment, naive CD4+ T cells lack repressive epigenetic marks associated with the IL4 locus[324, 347, 377-380, 385], and contain low levels of GATA-3 that are thought to be sufficient for the early initiation of IL-4 production[325, 422]. In vitro studies have identified that within the first few hours of TCR ligation, IL-4 and GATA-3 mRNA transcription are observed, even in STAT-6-deficient cells[327, 347, 348, 350]. Multiple rounds of culture in Th0 conditions can permit 10-fold increases in IL-4 protein production by antigen-stimulated IL-4R $\alpha$-/- lymph node cells[421]. Similarly, STAT-6-deficient $\mathrm{T}$ cells can be induced to produce IL- 4 and stably polarise to the Th2 subset for a number of weeks[348]. These findings illustrate that in the absence of IL-4R $\alpha$ or STAT-6, naive CD4+ T cells can begin to develop Th2 function in vitro.

Some factors that contribute to this IL4-independent expression of Th2 genes have been identified. Weak TCR ligation in the presence of IL-2 triggers the phosphorylation of STAT-5, which induces transcription of IL-4 mRNA and production of IL4[325, 349, 422]. GATA-3 remains important for IL-4-independent Th2 development in vitro because in its absence STAT-5-induced IL-4 production is defective[423]. Additionally, while STAT-6-deficient cells can become Th2 polarised, the Th2 cells that do develop Th2 function accumulate high levels of GATA-3 mRNA[348]. Therefore, STAT-5 promotes IL-4-independent IL-4 production in the presence of GATA-3. The ability of low-level TCR stimulation to promote CD4+ T cell IL-4 production is also observed in polyclonal systems, because when $\mathrm{T}$ cells with high-affinity for antigen are removed from culture, IL-4 production induced by high-dose antigen stimulation is increased[424]. Therefore, IL-2 and weak TCR stimulation induce IL-4-independent Th2 cell development in vitro.

During primary in vitro stimulation, the IL-4-independent phase of Th2 cell development is short. After the first 24 hours of TCR stimulation, exogenous IL- 4 is required 
to increase and maintain both IL-4 mRNA and GATA-3 mRNA transcript levels in vitro[349]. Additionally, while STAT-6-deficient cells can produce IL-4, and stably polarise to the Th2 subset, they do not reach the levels of polarisation that can be observed in STAT-6-sufficient Th2 cells[1,348]. These in vitro studies indicate that while IL-4 is not necessary during the initial activation phase in vitro, it remains a potent Th2-polarising cytokine. Thus, the initial transcription of IL-4 mRNA that occurs in vitro occurs in an IL-4-independent manner, and is followed by an IL-4-dependent phase that amplifies in vitro Th2 polarisation.

\subsubsection{Potential mechanisms that promote IL-4-independent Th2 cell development in vivo}

Understanding the initial events in IL-4-independent Th2 cell development in vivo is only beginning to be investigated. For a long time, it was thought that IL-4 was required for the initiation of Th2 cell development in vivo. When naive CD4+ $\mathrm{T}$ cells are initially activated, they can produce minute amounts of IL-4[421]. Similarly, NK-T cells and basophils have been shown to produce IL-4 in an IL-4- and STAT6-independent manner[357, 363, 421, 425]. Either of these cell populations could have been a source of early IL-4 that promoted Th2 cell development. However, numerous studies, including the current investigation, indicate that the initiation of Th2 cell development in vivo cannot be attributed to IL-4 alone. Therefore, rather than initiating Th2 cell development, these sources may instead contribute to the expansion of Th2 cells.

A number of potential Th2-initiating pathways have been proposed. Basophils have been implicated in the inititation of Th2 responses, both through their production of IL-4 and by presenting low levels of antigen-MHC-II[353, 354, 426, 427]. TSLP, IL-25 and IL-33 have also been shown to promote Th2 immunity through the development of previously uncharacterised basophil-like and lineage-negative Th2-cytokine producing populations[355, 428-433]. However, Th2 cells develop to normal levels in the absence of basophils[357-359] and the tissue-derived cytokines still enhance Th2 cytokine production in the absence of CD4+ T cells[432]. Epithelial cell production of IL-25 exacerbates allergic airways disease in mice and also promotes Th2 cell develop- 
ment in vitro; however, the effect of IL-25 specifically on Th2 cells requires IL-4[356]. Therefore, further investigations are required to determine the role of tissue-derived cytokines and basophils in the initiation of Th2 cell development. Specialised dendritic cell subtypes, namely those expressing FceRI or OX40L, have also been associated with the initiation of Th2 cell development[351, 359, 434]. In in vitro studies, OX40L also promotes CD4+ T cell IL-4 production[435, 436]. These processes may not require IL-4 to initiate Th2 cell development; this also requires further investigation. Investigating the earliest events that promote Th2 cell development may provide novel ways to interfere with the allergic process.

\subsubsection{Potential implications on IL-4-dependent Th2 cell ex- pansion}

Understanding the manner in which IL-4 increases the number of GFP+ or hCD2+ cells may have important implications for the aetiology of allergic disease. IL-4 may act to increase the proportion of committed Th2 cells that produce IL-4; IL-4 may induce the expansion of the committed Th2 cells that developed in an IL-4-independent manner; or IL-4 may promote polarisation of uncommitted antigen-specific Th cells towards the Th2 phenotype.

If IL-4 increases the proportion of committed Th2 cells that produce IL-4, the composition of the Th2 population would not be altered. According to the probabilistic regulation theory, the relative 'strength' of the Th2-inducing stimulus will cause more frequent IL-4-production by cells in a Th2 cell population[273, 401]. In this case, it is possible that the presence of IL-4 increases the Th2-activating 'strength' of an immunogen, and thus causes more committed Th2 cells to produce IL-4. Therefore, the number of committed Th2 cells will not be altered, but our ability to detect them will (Figure 4.17A). Thus, the composition of the antigen-specific population may not be altered, and their polarisation toward the Th2 phenotype may not be influenced. IL-4 may simply increase the proportion of committed Th2 cells that produce IL-4.

IL-4 may alternatively have altered the expansion of the allergen-specific Th cell population. The bulk Th2 cell population at the peak of the response could have 
developed from precommitted IL-4-independent Th2 cells (Figure 4.17B), or from previously uncommitted Th cells that became Th2 polarised (Figure 4.17C). These two scenarios will influence the clonal diversity of the Th2 cell population: If IL4 polarises uncommitted Th cells, then the Th2 cell population will become more clonally diverse; in contrast, if IL-4 acts solely to expand pre-committed Th2 cells, then the clonality of the Th2 cell population will not change, but the bias of the Th population towards the Th2 phenotype will be increased. At first sight, this may seem a redundant distinction. However, IL-4 production by CD4+ T cells has been shown to be preferentially invoked by low-dose and weak antigen-TCR stimulation[349, 424]. If IL-4 polarises uncommitted Th cells to the Th2 subset, this provides the potential for Th2 polarisation of Th cells with higher affinity for the insulting antigen. This, in turn could promote IgE class-switching of the affinity-maturing B cell population and contribute to the generation of high-affinity IgE. If instead, the pre-committed Th2 population is expanded by IL-4, identifying the manner in which IL-4-independent Th2 cell development is initiated could identify previously unexplored avenues for therapeutic intervention.

Basic mechanistic studies offer support to both types of Th2 population expansion. Recent studies indicate that STAT-6 phosphorylation is observed in essentially every non-Th2 cell in draining lymph nodes of mice infected in the foot-pad with H. polygyrus bakeri[334]. IL-4Ra is downregulated on highly-activated Th2 cells[373], rendering them refractory to the phosphorylation of STAT- 6 caused by IL-4 produced in a draining lymph node[334]. If IL-4 directly induces Th2 cell development, this could indicate that the IL-4 produced by Th2 cells induces IL-4 production by previously uncommitted cells. In the work by Perona-Wright et al.[334], when foot-pad-infected mice subsequently received OVA-peptide-loaded dendritic cells, OVA-specific CD4+ $\mathrm{T}$ cells in the foot-pad draining lymph node were preferentially polarised to the Th2 subset[334]. Therefore, Th2 polarisation of uncommitted Th cells is possible, and can induce Th2 polarisation of unrelated allergens.

While activated Th2 cells may be refractory to autocrine IL-4[334], their expansion may occur indirectly through interaction with accessory cells. As established by others $[275,362,364]$, we observed that a large proportion of Th2 cells were TFH cells (Figure 4.7 and 4.8). It is known that CXCR5-expressing TFH cells migrate into 
B cell follicles[337, 437], and that B cells can provide stimulatory signals to antigenexperienced CD4+ T cells[438]. Therefore, it is plausible that committed Th2 cells, despite being refractory to IL-4, interact with B cells, expand and continue to produce IL-4 (Figure 4.17A). Equally, it could be argued that TFH arise from the uncommitted Th population after they are exposed to IL-4. However, while TFH populations take time to expand fully[364], the bifurcation of cells that are likely to become TFH cells (and by proxy a reasonable proportion of Th2 cells) or non-TFH Th cells can be identified within three days of immunisation[368]. Interaction with dendritic cells alone is also sufficient to initiate TFH development[368, 439], and similarly to initiate IL-4 production[408]. In the current study, we identified no requirement for IL-4 for Th2 cell development in the first three days (Figure 4.2 and 4.3). Taken together, it is plausible that IL-4-independent Th2 cells may be preferentially selected as the Th2 cell population prior to the involvement of IL-4 in Th2 cell expansion.

One final point we must consider is that IL-4 signalling may influence the allelic expression of IL-4, inducing more biallelic expression. We have based our studies on comparisons made in heterozygous $\mathrm{G} 4 /+, \mathrm{KN} 2 /+$ and $\mathrm{KN} 2 / \mathrm{G} 4$ mice. In our in vitro studies, we observed a disproportionate increase in biallelic IL-4 production in cells exposed to IL-4 (figure 3.3 and 3.4). In these comparisons, KN2/+ and KN2/G4 cells cultured in IL-4 had similar frequencies of hCD2 expression as KN2/KN2 cells, indicating that IL-4 may have influenced the allelic expression profile. While the differential was maintained in G4/+ and G4/G4 cultures in vitro, it is possible that IL-4 potentiated both G4/+ and $\mathrm{KN} 2 /+$ Th2 cells to express IL-4 biallelically in vivo. However, the concordance between the two reporter systems suggest that even if IL-4 is 'potentiating' biallelic expression, this 'potentiation' of Th2 cells may be an important component of Th2 development. Future studies will be required to clarify the role of IL-4 in IL-4-producing Th2 cell development.

The manner in which IL-4 increases Th2 development may have important implications for allergic disease biology. If pre-committed Th2 cells expand or produce IL-4 in response to IL-4, understanding the processes that initiate IL-4-independent Th2 cell development may provide new prophylactic disease interventions. If IL-4 polarises uncommited Th cells to the Th2 phenotype, then targeting antigen-responsive cells, rather than the Th2-polarising apparatus, may provide more therapeutic benefit. 
Further, if IL-4-independent Th2 cells are long-lived, and remain IL-4-independent, then interfering with IL-4 signaling may limit, rather than reverse the disease phenotype. I think the most likely explanation is that IL-4 increases the polarisation of uncommitted Th cells to the Th2 phenotype; however, clarifying the mechanism that underlies IL-4-dependent Th2 cell expansion will be an important endeavour for future studies. 


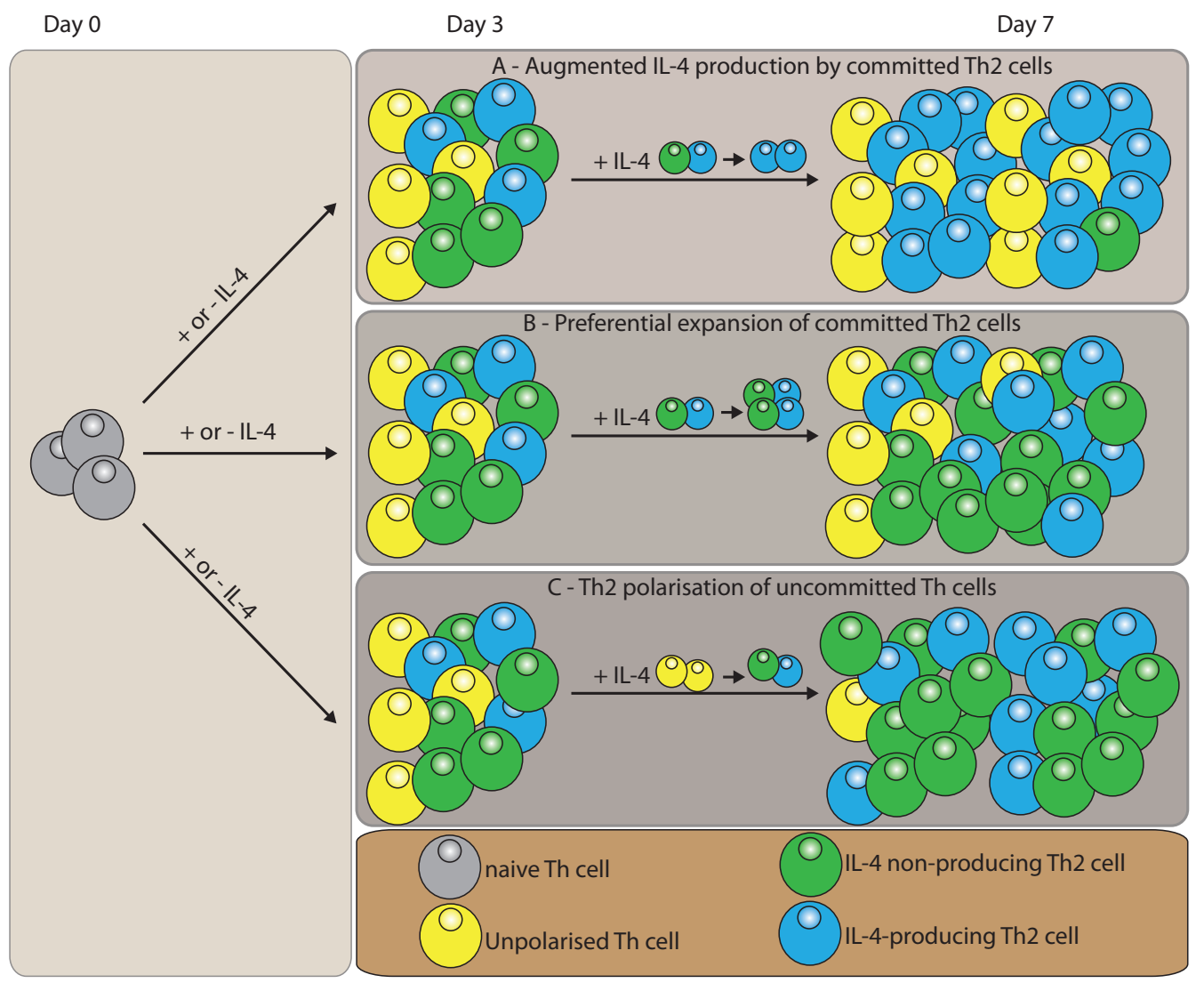

Figure 4.17: Potential mechanisms of IL-4-dependent Th2 cell expansion. (A) IL-4 may increase the proportion of committed Th2 cells that produce IL-4 without changing the proportion of Th2-committed cells. (B) The presence of IL-4 may result in the preferential expansion of committed Th2 cells without altering the balance of IL-4-producing and non-producing Th2 cells. (C) IL-4 may polarise uncommitted Th cells towards the Th2 phenotype. 


\subsubsection{A glimpse at the allelic expression of IL-4 in vivo}

In addition to operating as an IL-4-deficient single-allele reporter, the KN2/G4 reporter mice also provided insight into the allelic expression profile of IL-4 in vivo. This provides novel understanding of the potential phenotype of the Th2 cell. In response to both dead $i$ L3 $N$. brasiliensis and HDM immunisation, a small proportion of cells were positive for both hCD2 and GFP (Figure 4.4 and 4.8). Previous explorations have also identified that dual 4get/KN2+ cells develop in helminth-infected mice[275, 362] and in response to isolated helminth antigens[364]. Similarly, GFP + CD4 + T cells isolated from helminth-infected G4/+ cells contain elevated levels of IL-4 mRNA[1]. Thus, in two systems, biallelic IL-4 expression has been determined at the mRNA level. Our studies extend these findings and confirm that the Th2 cells that developed were able to produce IL-4 biallelically. We also observed that biallelism was rare. Our observation of predominant monoallelism in vivo is in accordance with our in vitro observations, and in agreement with previous in vitro studies[273, 352, 374, 388, 401, 403]. The mice that we monitored for the development of the biallelic population were IL4-deficient, indicating that biallelic expression of IL-4 also did not require IL-4 to be produced. Therefore, Th2 cells that develop in the absence of IL-4 can produce IL-4 both mono- and bi-allelically in vivo.

The allelic expression profile of IL-4 in vivo is a potentially important aspect of the Th2 response, and we conceived that it may have demarcated functionally specialised Th2 cells. When we tested whether biallelism specifically demarcated TFH cells, while we observed enrichment in the biallelic population, we also observed strong enrichment in the hCD2 + monoallelic, and to a lesser extent, the GFP + monoallelic population (Figure 4.8). This indicated that while both TFH development and biallelism were rare, biallelism was not a specific attribute of Th2 TFH cells. Future investigations will be required to determine whether there are functional differences between cells producing IL-4 mono- or bi-allelically. 


\subsubsection{Modulating IL-4 levels in Th2 immunity}

Following our investigations into the role of IL-4 in Th2 cell development, we examined the influence of IL-4 heterozygosity on allergic Th2 immune responses. In these studies, we designed experiments to evaluate how the loss of one IL-4 allele affected IL-4-dependent immune responses.

In these experiments, we first explored the B cell-helper potential of pTh2 cells. First, we clarified the phenotype of pTh2 cells. Our studies demonstrated that 10 to $30 \%$ of pTh2 cells were TFH cells, and some of these had a germinal centre TFH phenotype ([366]; Figures 4.6, 4.7 and 4.9). Thus, pTh2 cells had the potential to support B cell class-switch responses. We then confirmed that germinal centre B cells expanded (Figure 4.9), and that IgG1 class-switching was observed in intradermally immunised mice (Figure 4.10). This was associated with the development of systemic antigenspecific IgG1 in mice after immunisation (Figure 4.10 and 4.11). Finally, we confirmed that the IgG1 class-switch response was indeed STAT-6- and IL-4-dependent, as would be expected based on established data ([1, 289, 329-331]; Figure 4.12 and 4.13). Our studies established that the pTh2 assay is a suitable assay in which to examine IL-4dependent IgG1 class-switch responses.

\section{Primary Th2 cell development and IgG1 class switch recom- bination}

While it is not directly related to our investigations into the role of IL-4, our studies in wild-type IgG1 class-switch responses are worthy of a brief digression. In these studies, we observed concordance between Th2 cell development and the level of IgG1 switching induced by two different immunogens. When dead $i$ L3 N. brasiliensis larvae were used as the immunogen, more IgG1-switched cells were detected in the local lymph node than when HDM was used (Figure 4.10). Similarly, more Th2 cells developed in response to dead $i$ L3 $N$. brasiliensis larvae than to HDM (Figure 1.4). Taken together with the TFH phenotype established as IgG1-switch inducing $\mathrm{T}$ cells[275], this suggests that class switching occurs in proportion with the number 
of Th2 cells an allergen causes to develop. Exploring this correlation with a range of immunogens will be required to validate these findings, but this suggests that at least two aspects of the Th2 response can be observed by primary intradermal immunisation: Th2 cell development and IL-4-dependent class switching.

In addition to IgG1 switching, it will be advantageous in the future to examine $\operatorname{IgE}$ switching in this setting. While both IgG1 and IgE require IL-4 for production, IL-21, which is produced by TFH cells[275, 367], can have reciprocal effects on these two isotypes[440-443]. In our studies, IgG1 titres were only elevated significantly to titres of $1 / 40$ or $1 / 80$ (figure 4.11 ). IgE is produced at $1000-2000$-fold lower concentrations than IgG1[287], making it unlikely that we would detect systemic antigen-specific IgE in intradermally-immunised mice. Similarly, detecting IgE class-switch recombination is beyond the sensitivity of most current methodologies; however, newly developed IgE-reporter technologies are shedding light on the processes that lead to IgE production in vivo[444, 445]. These may permit the detection of the IgE-inducing capacity of immunogens in a modified pTh2 assay. Therefore, future investigations will therefore require examination of a larger range of allergens and will also need to determine if IgG1 and IgE correlate with Th2 cell development, or alternatively determine if they reflect alternate allergenic properties of immunogens.

\subsubsection{IL-4 haplo-insufficiency in Th2 immunity}

\section{IL-4 heterozygous mice and IgG1 class-switch recombination}

To evaluate the requirement for two intact IL-4 alleles in allergic Th2 immune responses, we examined the IgG1 class-switch response in the pTh2 assay, and subsequently examined the disease phenotype of $+/$ - mice in an oral allergen-induced model of food allergy. When we examined IgG1 class-switching, we observed that the loss of one IL-4 allele decreased allergen-induced IgG1 class-switching by approximately $50 \%$ (Figure 4.13A). +/- mice were also shown to have a $90 \%$ impairment in adjuvant-based $\operatorname{IgE}$ generation, as observed previously ([1]; Figure 4.14). +/mice were also protected from oral allergen-induced diarrhoea in the intestinal allergy model (Figure 4.16). These findings indicated that both alleles of IL-4 were required 
for normal anti-allergen Th2 immune responses. IL-4 heterozygous mice are therefore haplo-insufficient for IL-4.

Current models of antibody production indicate that the manner in which $\operatorname{IgE}$ is generated differs from the other major isotypes. In vitro studies indicate that in contrast to B cells of the $\operatorname{Ig} \mathrm{A}$, IgM and IgG isotypes which can expand 5-15-fold, IgE-producing B cells have essentially no capacity to expand once they are induced to become an IgE-producing cell[284]. These studies are also supported by recent in vivo studies suggesting that once B cells class-switch to IgE they rapidly differentiate into short-lived plasma cells at a reasonably conserved ratio across a progressing $\mathrm{N}$. brasiliensis infection or following haptenated protein/alum immunization (PC:GC B cell ratio close to 1:1)[445]. This contrasts with IgG1 class-switched B cells, which become preferentially accumulated in the germinal centre niche (PC:GC B cell ratio initially approximately 1:5 and later approximately 1:5000)[445]. Furthermore, as studies of the secondary immune response using haptenated proteins or N. brasiliensis infection reveal that IgE producing cells arise from IgE-negative memory B cells in an IL-4 and T cell dependent manner[274, 287, 288, 306, 407], there is evidence that even in secondary immune response contexts, $\operatorname{IgE}+\mathrm{B}$ cells have limited ability to clonally expand.

The concept of a short-lived, poorly expanding $\operatorname{Ig} \mathrm{E}+\mathrm{B}$ cell also has implications for the processes that select a B cell into the IgE producing plasma cell population. While serum IgE affinity increases with time and following boost immunization[306], this is not necessarily because the IgE-switched B cells are undergoing clonal expansion, but could be a result of their continual loss and subsequent rederivation from IgE negative antigen-specific B cells. Following haptenated protein immunization, there is evidence of less affinity maturation in IgE + plasma cells than IgG1+ plasma cells, but still a high frequency of the IgE + cells contain germline transcripts associated with high antigen affinity, indicating that a substantial proportion are generated through the extrafollicular pathway[445]. However, there are temporal increases in the number of accumulated mutations and inferred affinity of $\operatorname{IgE}$ producing plasma cells[306, 445], and at later timepoints a substantial proportion of these high affinity IgE+ B cells contain sterile gamma-1 switch transcripts indicating that they have passed through an IgG1 intermediate state $[279,306]$. Thus, current models suggest that 
$\operatorname{IgE}+\mathrm{B}$ cells develop both via the extrafollicular route and during the germinal centre reaction.

In our studies, immunization with OVA/alum generated much less IgE in G4/+ mice than in wildtype mice (Figures 4.14 and 4.15). Further, intradermal immunization with HDM demonstrated that IgG1 class-switching was also decreased (Figure 4.13). Intriguingly, anti-IL-4 treatment inhibits T-dependent IgE production in both primary and secondary immunization contexts, but does not suppress secondary immunizationinduced T-dependent IgG1 production[287]. Further, while IgE production following anti-IgD immunization is STAT-6 and IL-4 dependent, anti-IgD induced IgG1 production does not require STAT-6 or IL-4[287, 329, 331]. This indicates that the production of IgE is more sensitive to defects in IL-4 than is IgG1 production. It is possible that IL-4 is one of a number of key mediators of the IgG1 class switch, but it is an irreplaceable mediator of the IgE class switch. Mechanistically, this could relate to differences in the amount of IL-4 required to promote IgG1 versus IgE production. In vitro, lower concentrations of IL-4 are required to initiate IgG1 production than IgE production[446], which could indicate that a certain threshold level of IL-4 is required for IgG1 and a higher threshold level is required to generate $\operatorname{IgE}$ in vivo. In support of this, our id HDM immunization data, in which the magnitude of IgG1 production was approximately 100-fold lower than that induced by OVA/alum immunization (antigen-specific IgG1 titres of approximately 1/1000 vs. 1/100000) showed that draining auricular lymph node IgG1 class-switching was decreased in the G4/+ mice compared to wildtype mice. Alternatively, it is also possible that IL-4 heterozygosity reveals a role for IL-4 in the initial induction of IgG1 class-switching in germinal centre B cells, but the expansion of this population thereafter was of sufficient magnitude to mask any measurable effect following protein/alum immunization. As IL-4 signaling has established roles in IgG1 affinity maturation [275], it also remains possible that even with protein/alum immunization the absence of one IL-4 allele has some uncharacterized impact on the quality of the IgG1 response.

In contrast to the IgG1 class-switch, our data indicate that IgE production was sensitive to the amount of IL-4 available at all stages, because IgE production was impaired in G4/+ mice immunized with OVA/alum. As current models suggest that IgE classswitched B cells have limited capacity to expand, the heterozygous cells appear to 
be unable to secrete sufficient IL-4 to induce B cells to class-switch to IgE at normal frequencies. As previously mentioned, this may relate to the sheer amount of IL-4 available, or alternatively, could indicate that $\operatorname{IgE}$ switching requires highly concentrated synaptic production of IL-4 at the interface between cognate conjugated CD4+ T cells and B cells (i.e., at the immunological synapse). In support of this latter concept, IL-4R-alpha heterozygosity impairs IgE production by approximately $90 \%$ yet does not impinge upon IgG1 production[447]. Together with our findings, this could indicate that $\mathrm{B}$ cells require either frequent or strong IL-4 receptor stimulation to class-switch to IgE and this can only be provided by CD4+ T cells with biallelic IL-4 producing potential.

While it seems likely from current models that the class-switching to epsilon is impaired in the +/- mice, there are some alternate explanations to the data. Classswitching does not guarantee a B cell will develop into an antibody producing cell[282, 283, 448]. Further, there are reported instances of IL-4-independent IgE production[416, 418] and elevated epsilon mRNA transcript frequencies in IL-4R-alpha-deficient mice[360]. While we consider it to be a less likely scenario, epsilon class-switching may occur normally in the IL-4 +/- mice but these cells may have impaired ability to enter the plasma cell pathway, or require high levels of IL-4 to survive. Finally, it is also possible that both alleles of IL-4 are required to generate the precursor population from which the IgE producing B cells derive. Again, because a substantial proportion of IgE+ B cells have passed through an IgG1 intermediate state[306] and IgG1 titres could be generated in the IL- $4+/$ - mice, this seems to be unlikely, but remains a possible source of the diminished IgE production we observe. Collectively, these findings have implications for the manner in which an IL-4-producing CD4+ T cell can facilitate IgE production.

\section{Modulating IL-4 levels in other models of Th2 immunity}

While the role of IL-4 has been studied many times in IL-4-deficient conditions, studies in which IL-4 levels are modified are less common. Over-expression studies indicated that IL-4 results in severe auto-immune pathologies, were associated with atopic dermatitis-like lesions, hyper-IgE production and IL-4- and CD4+ T cell dependent 
self-reactive B cell responses[404, 405]. More recently, mutations in genes that result in over-production of IL-4 have been shown to induce similar atopic dermatitis-type phenotypes and hyper-IgE production[449]. Regulating the amount of IL-4 produced has key implications for the atopic/allergic process. The closest comparative study to the current investigation comes from work done by Kopf et al., and the preceding body of work by vanPanhuys et al. in IL-4-heterozygous mice[1, 450]. Kopf et al. examined the effect of IL-4 heterozygosity on Leishmania major infection in mice[450]. Despite no requirement for IL-4R $\alpha$ in Th2 cell development[420], in the absence of IL-4 protein Kopf et al. observed that footpad swelling in disease-susceptible BALB/c mice was minimised in response to footpad inject with L. major promastigotes[450]. Similarly, footpad swelling in $+/$ - mice was reduced, but not ablated[450]. van Panhuys et al., in addition to comparing Th2 cell function in $+/+$ and $\mathrm{G} 4 /+$ mice, observed $50 \%$ less IgE in anti-IgD or $N$. brasiliensis-infected mice, and a $90 \%$ reduction in aluminduced IgE production[1]. Therefore, IL-4 heterozygosity resulted in reduced disease severity in L. major-infected mice, and decreased, but did not ablate IgE production in IL-4-heterozgyous mice. We confirmed that IL-4-dependent Th2 responses require both IL-4 alleles.

\section{Determining the amount of IL-4 available locally in IL-4-heterozygous mice}

Assessing the amount of IL-4 available during local immune responses in IL-4 heterozygous conditions was recently investigated based on the expression of molecules by IL-4exposed cells. In these studies, in vitro IL-4 concentrations above $15 \mathrm{pg} / \mathrm{mL}$ increased IL-4R $\alpha$ expression on cultured non-Th2 cells in a STAT-6-dependent manner[334]. However, when IL-4 concentrations exceeded $500 \mathrm{pg} / \mathrm{mL}$, both STAT-6+/+ and STAT-6-/- cells downregulated IL-4R $\alpha$ expression[334]. By examining the expression of IL-4R $\alpha$ on non-Th2 cells in helminth-infected chimeric mice, they observed IL-4R $\alpha$ upregulation in STAT-6+/+ cells, but not downregulation in STAT-6-/- cells[334]. From these data, Perona-Wright et al. were able to conclude that the effective concentration of IL-4 in the local lymph node was equivalent to 15 to $500 \mathrm{pg} / \mathrm{mL}$ in vitro units[334]. In our local lymph node studies in +/- mice, it may be similarly possible 
to use IL-4R $\alpha$ expression to approximate the effective interstitial IL- 4 concentration. Alternatively, examining IL-4 mRNA transcript numbers, as done previously in IL$4 \mathrm{R} \alpha$-deficient conditions[360] may allow us to approximate the relative defect in IL-4 transcription in IL-4 haplo-insufficient conditions.

\subsubsection{Mechanisms that may underlie the haplo-insufficient phenotype of IL-4-heterozygous mice}

While we observed impaired IgG1 class switching in +/- mice, at least two mechanisms may underlie this phenotype. Polarised Th2 cell development may have been impaired in $+/$ - mice relative to $+/+$ mice. Alternatively, a single IL-4 allele may have been sufficient to permit normal Th2 cell development, but their ability to produce IL4 may have been compromised. Based on the current data, we cannot distinguish between the two.

Th2 cell development may have been compromised in +/- mice. Our studies comparing G4/+ and KN2/G4 mice determined that IL-4 was required for the normal expansion of the Th2 cell population(Figure 4.2 and 4.3). However, we are unable to compare $+/$ - and $+/+$ mice in the same manner. Different aspects of the Th2 response are sensitive to differences in the concentration of IL-4 to which cells are exposed. For example, in vitro studies show that 1000-fold lower concentrations of IL-4 are required to induce IgG1 class-switching than that to $\operatorname{IgE}[446]$. Similarly, lower concentrations of IL-4 are required to induce MHC-II expression than IgG1 class switching of mitogen-activated B cells in vitro[288]. In our studies, we observed MHC-II upregulation, but impaired IgG1 class-switching in HDM-immunised +/mice (Figure 4.13). While these in vitro experiments were very different to our in vivo system, this indicates that IL-4 was available in $+/$ - mice, but was at decreased levels compared to $+/+$ mice. It is possible that the amount of IL- 4 required for normal expansion of the Th2 cell population also requires lower IL-4 concentrations than IgG1 switching. It may be possible to determine whether Th2 cell development is also impaired by examining mRNA transcript levels of other Th2 cytokines, like IL-5, or examining the development of Th2 cells using a reporter that identifies IL-4 producing cells without interfering with the wild-type genes. Bacterial artificial chromosomes 
have been used in the past to report Th2 (IL-4) activity[383]. Similarly, using a BAC transgenic system crossed to $+/+,+/-$ and -/- conditions could accurately determine how the loss of each allele affects Th2 cell development in vivo. A similar system is being explored in our lab (Ryan Kyle, personal communication).

It is possible that IL-4 by TFH cells was impaired and Th2 cells developed normally. Deficient IgG1 switching and IgE production could be caused by functional defects in the TFH compartment. TFH were shown, in this and earlier studies, to be enriched in IL-4 producing cells[275, 362, 364]. IL-4-producing cells have also been shown in vivo to be directly conjugated to IgE class-switched and IgG1 affinity-matured B cells[275]. Secretion of IL-4 at the immunological synapse, although not exclusively directed towards the site of interaction[451, 452], may increase by many times the concentration of IL-4 to which cognate B cells are exposed. Understanding how genetic lesions in IL-4 production impact Th2 development and B cell responses could clarify some of the most important events that lead to allergic sensitization.

\subsubsection{The effect of IL-4 heterozygosity on the intestinal al- lergic process}

Allergic mastocytosis was also impaired in IL-4+/- mice. In the model we used, allergic mastocytosis and diarrhoea require IL-4 signaling[295]. A number of studies demonstrate that local intestinal elevation of IL-4 is associated with diarrhoea development in mice[37, 38, 57, 299]. Studies also demonstrate that adoptive transfer of Th2 cells from the mesenteric lymph nodes of mice with diarrhoea can transfer diarrhoea to naive mice[38]. It is plausible that the loss of a single IL-4 allele reduced CD4+ T cell-derived IL-4 from reaching sufficient levels to promote intestinal mastocytosis. However, Th2-associated cytokines other than IL-4 are also implicated in the intestinal allergic response[37, 57, 295], or can enhance parasite-induced intestinal mast cell expansion[309, 311, 315]. Therefore, it is also possible that Th2 cell development was impaired, resulting in depressed production of other mast cellactive cytokines. A third explanation is also possible. IgE can activate mast cells and prolong their survival[30, 45, 453]. In our studies, we observed that alum-based immunisation resulted in a greater than $90 \%$ reduction in systemic IgE titres, both 
prior to- and after- allergen challenge (Figure 4.14 and Figure 4.15). Therefore, it remains possible that the apparent defect in oral-allergen induced intestinal mastocytosis was indirect. Thus, we cannot definitively conclude whether the lesion was caused by impaired Th2 cell development, impaired Th2 cell function or impaired IgE production. Clarifying the level at which the impairment in mastocytosis arises has the potential to dissect specific functions of Th2 cells, and specific roles of IL-4 in the full allergic process. It will be useful to further clarify the role of IL-4 in this allergic process in future work.

While CD4+ Th2 cells are implicated in disease development, it also remains possible that the disease was dependent on IL-4 from other cellular sources. Numerous other cell types can produce IL-4[300-305, 363, 425] and, therefore, may contribute to the elevation in Th2-type cytokines that permit jejunal mastocytosis. While the parsimonious explanations remain that either local Th2 cytokine levels were too low to permit disease, or inadequate IgE was generated to sensitize mast cells, clarification of the role of $\mathrm{CD} 4+\mathrm{T}$ cells in the intestinal allergic process is required.

Collectively, our investigations highlight the importance of IL-4 in the allergic process. We observed a contributory role for IL-4 in adjuvant-free Th2 cell development. We further determined that, like mice lacking both IL-4 alleles[295], missing one allele impaired IL-4-dependent antibody production, inhibited oral allergen-induced mastocytosis and ultimately prevented allergen-induced intestinal anaphylaxis. Determining which aspect causes the largest lesion could help us understand whether targeting IgE, targeting IL-4 or targeting allergen-reactive Th2 cells will provide a better long-term treatment for allergic disease.

\subsection{Summary}

The pTh2 assay revealed that IL-4 was able to amplify Th2 cell development in a STAT-6-dependent manner. The loss of a single allele of IL-4 impaired the B cell class-switch response to IgG1 in IL-4 heterozygous mice immunised intradermally. IL-4 heterozygosity also caused an impairment in adjuvant-based IgE production and the subsequent intestinal allergic response. 



\section{Chapter 5}

\section{Establishing an allergy-permissive environment through allergen exposure in the gut}

\subsection{Introduction}

\subsubsection{Assessing the role of $\mathrm{CD} 4+\mathrm{T}$ cells in the post-sensitization phase of food allergy}

In 2003, Brandt et al.[37], developed an IL-4-, mast cell-, and IgE-dependent model of oral allergen-induced intestinal anaphylaxis[37, 295]. In this system, mice are first sensitized to OVA by intraperitoneal injection of OVA plus alum adjuvant, and are then orally challenged with intragastric (i.g.) doses of OVA every second day, eventually resulting in acute and transient diarrhoea that resolves 120 minutes after oral challenge. One of the intriguing aspects of this system is that multiple allergen challenges are required before mice develop diarrhoea. This indicates that oral allergen challenge of sensitized mice initiates a cascade of inflammatory events that establish the appropriate inflammatory milieu to permit subsequent disease triggering. Interestingly, oral allergen challenge induces jejunal mastocytosis and increases intestinal- and systemicIgE production, but when jejunal Th2 cytokine levels are not elevated, disease development is inhibited[299]. That is, there is a local inflammatory process invoked by oral allergen challenge that precedes, but does not guarantee, the development of the intestinal allergic response. I will refer to this process as oral potentiation, but more accurately, it is the establishment of a Th2-associated inflammatory environment that greatly exacerbates (and in the model employed, is required for) intestinally-evoked 
allergic responses to orally administered allergens in previously sensitized mice.

In addition to the outlined findings, further evidence implicates intestinally-localized CD4+ T cells and Th2-type cytokines in the oral potentiation process [38, 57, 58, 295, 298, 299]. Prior to diarrhoea onset, sequential i.g. OVA challenges increase jejunal IL4 levels in wild-type mice[37], promotes IL-4 production by intestinal CD4+ T cells[57, $58]$, and triggers proliferation of- and $\alpha 4 \beta 7$ expression on- OVA-specific CD4+ $\mathrm{T}$ cells[38]. Adoptive transfer of OVA-restimulated CD4+ T cells from mesenteric lymph nodes of diarrhetic mice transfers disease susceptibility to naive mice[38]. CD4+ Th2 cell responses are also observed in the intestinal tissue of allergic individuals in the clinic[90, 454]. To more closely dissect the involvement of CD4+ T cells in local oral potentiation, we investigated how their depletion at different stages of the postsensitization allergic process affects disease progression.

Much is still unknown about the immune molecules that govern allergic mastocytosis. This is an important issue, because the in the mouse, severity of the orallyinduced anaphylactic response correlates with the number of mast cells present in the intestine[36]. It is known that stem cell factor (SCF) is required for the presence of mast cells in the intestine at baseline[307], and IL-3, IL-4 and IL-9 amplify intestinal mast cell infiltration caused by parasite infection[311-313]. In agreement with this contributory role of these cytokines in the intestinal mast cell response, mice deficient in either IL-4 or IL-9 are protected from mast cell-dependent experimental allergic diarrhoea[57, 295]. Similarly, blockade of IL-4R signaling impairs disease development[295] and over-expression of IL-9 increases basal jejunal mastocytosis and promotes intestinal allergic disease[57]. However, it remains to be established whether IL-3 contributes to allergic intestinal mastocytosis and this may be a critical, previously uncharacterized component of the intestinal allergic process.

The experiments in this chapter aimed to clarify the role of CD4+ T cells and mast cell-active cytokines in the orally-induced allergic response. 


\subsection{Aims}

In this section, we investigate the role of CD4+ T cells and non-classical Th2 cytokines in the post-sensitization allergic process.

Our specific aims were:

1) To establish if CD4+ T cells are required for allergic diarrhoea;

2) To determine whether CD4+ T cells contribute to disease maintenance;

3) To elucidate whether IL-3 is involved in experimental food allergy. 


\subsection{Results}

\subsubsection{The role of $\mathrm{CD} 4+\mathrm{T}$ cells in oral potentiation}

Our initial experiments focused on the role of CD4+ $\mathrm{T}$ cells in allergic diarrhoea. It is established that Th2 CD4 $+\mathrm{T}$ cells are increased in intestinal tissues of orallychallenged mice[57, 58] and that adoptive transfer of CD4+ T cells can render naive mice susceptible to allergic diarrhoea[38]. While IgE and mast cells are critical mediators of oral allergen-induced diarrhoea[37], numerous studies demonstrate that the presence of IgE, and of mast cells, does not automatically render mice susceptible to disease $[57,67,299]$. We were therefore interested to determine whether CD4+ T cells were influencing disease susceptibility over and above their established contribution to systemic IgE generation[275, 289, 392].

We first sensitized mice, then subsequently depleted them of CD4 $+\mathrm{T}$ cells with GK1.5. Mice were then i.g. challenged with $50 \mathrm{mg}$ OVA and monitored for diarrhoea development. The sensitization, depletion and challenge regimen is shown (Figure 5.1A). We observed that sensitized, mock-depleted mice developed diarrhoea after 6-10 oral challenges, whereas sensitized-CD4-depleted mice were entirely protected from disease (Figure 5.1B). Sixty minutes after the final i.g. challenge, mock-depleted mice lacked colonic pellets and had voided caecae, which contrasted with the visible pellets and normal caecal content of CD4-depleted mice (Figure 5.1G). With sequential gavages we observed increases in total- and OVA specific-IgE titres in mockdepleted mice, which were prevented in CD4-depleted mice (Figure 5.1E and F). We also observed approximately 100-fold less mMCP-1 and reduced jejunal mastocytosis in CD4-depleted OVA-challenged mice (Figure 5.1C and D). This demonstrates that oral allergen-induced jejunal mastocytosis is CD4 $\mathrm{T}$ cell dependent. In a second experiment, we showed that although CD4-depleted mice were protected from diarrhoea they remained susceptible to i.v.-induced systemic anaphylaxis (Figure $5.2 \mathrm{~B}$ and $5.2 \mathrm{C}$ ). This was observed both with and without oral OVA challenge (Figure $5.2 \mathrm{~B}$ and $5.2 \mathrm{C}$ ). While some of the mice in the PBS-fed group succumbed to fatal anaphylaxis, further experiments would be required to validate whether this reflects a protective effect of oral challenge in the absence of CD4 $+\mathrm{T}$ cells. It was therefore 
specifically the development of an intestinal allergic response that was prevented by depletion of $\mathrm{CD} 4+\mathrm{T}$ cells. These data indicate that the development an intestinal allergic response was prevented by depletion of CD4+ T cells, however, systemic sensitization was maintained in the absence of CD4+ T cells. 


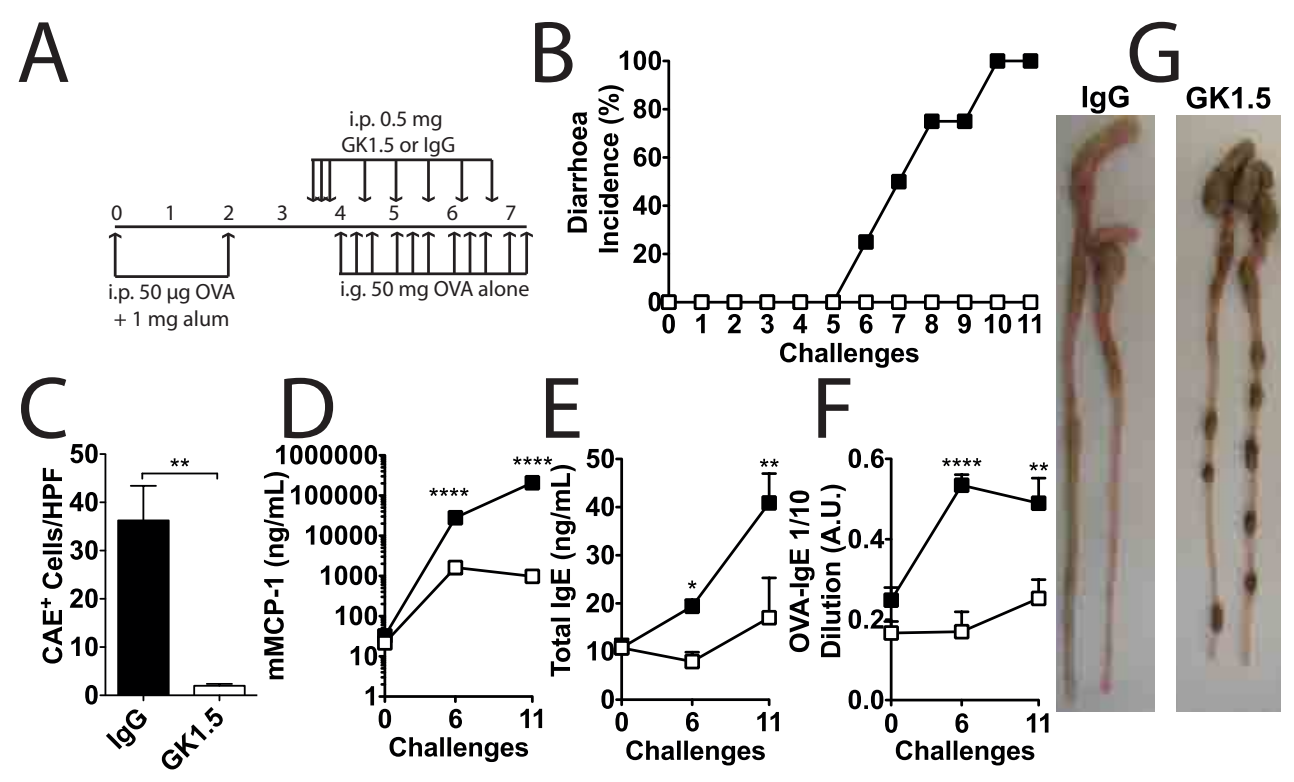

Figure 5.1: CD4+ T cell depletion prevented diarrhoea and oral potentiation. Mice ( $n=4$ /group) were sensitized to OVA, depleted of CD4+ T cells with GK1.5 or mock-depleted with Rat IgG and i.g. challenged with OVA as outlined in (A). (B) Diarrhoea development was monitored 45-90 minutes after i.g. OVA challenge of CD4+ T cell-depleted (white boxes) and mock-depleted (black boxes) mice. (C) Chloroacetate esterase stained cells (CAE+; mast cells) in jejunal sections collected 60 minutes after the final challenge. (D) Serum mMCP-1, (E) Total IgE and (F) OVA-specific IgE were analysed by ELISA after the stated number of i.g. OVA challenges. Data are represented as group mean \pm SEM. (G) Colons and caeca of mockdepleted (IgG) and CD4-depleted (GK1.5) mice were excised 60 minutes after the final challenge and photographed. Data are shown from a single experiment and were compared (C) using Student's t-test or (D-F) using Two-way ANOVA with Bonferroni's post-test. ${ }^{*} \mathrm{p}<0.05,{ }^{*} \mathrm{p}<0.01,{ }^{* * * *} \mathrm{p}<0.0001 . \quad \mathrm{mMCP}-1$ data were log-transformed prior to analysis. 

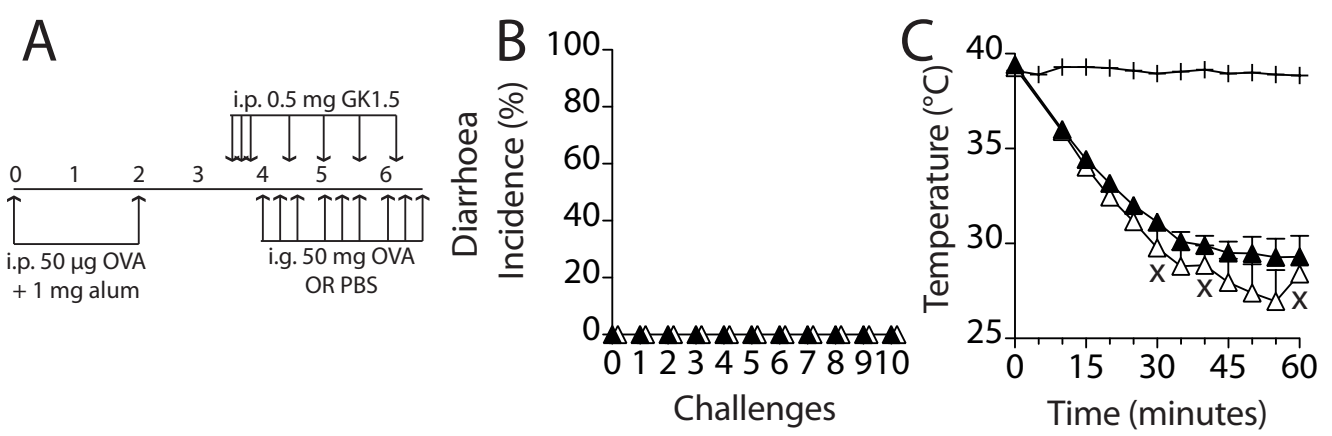

Figure 5.2: $\mathbf{C D} 4+\mathbf{T}$ cell depletion did not prevent systemic anaphylaxis. Mice ( $\mathrm{n}=4$ /group) were sensitized and depleted of CD4+ T cells and subsequently fed with OVA or PBS as indicated in (A). (B) OVA-fed mice (Black triangles) or PBSfed mice (White triangles) were monitored for diarrhoea development 45-90 minutes following each challenge. (C) 72 hours after the final gavage, mice shown in (B), or naive mice (plus symbols; $\mathrm{n}=2$ ) were i.v.-administered $100 \mu \mathrm{g}$ OVA and monitored for rectal temperature. Data are shown from a single experiment and points show mean \pm SEM of groups. $x$ symbols denote one death in the PBS-fed CD4-depleted group (three total). 
To determine whether short-term CD4+ $\mathrm{T}$ cell depletion conferred long-term protection from allergic diarrhoea, we depleted CD4+ T cells from sensitized mice and then allowed them to replenish during the oral challenge period. We observed that peripheral depletion lasted approximately two weeks (Figure 5.3A). During this time, we monitored mice for diarrhoea. Initially we observed that CD4-depleted mice were protected from allergic diarrhoea, however, diarrhoea eventually manifested in 60 percent of mice (Figure 5.3B). This correlated closely in time with the re-emergence of peripheral CD4+ T cells (c.f. Figure 5.3A and 5.3B). While we observed augmented OVA-specific antibody production in mock-depleted mice, but changes in antibody titres were negligible in the CD4-depleted group until they were CD4-replete (Figure 5.3C and 5.3D). Jejunal mastocytosis, serum mMCP-1 titres, and the amount of caecal washout were reduced in CD4-replete mice compared to mock-depleted mice (Figure 5.3E - G). Taken together, these findings indicate that CD4+ T cells play a central role in the development of the intestinally localized allergic response. 


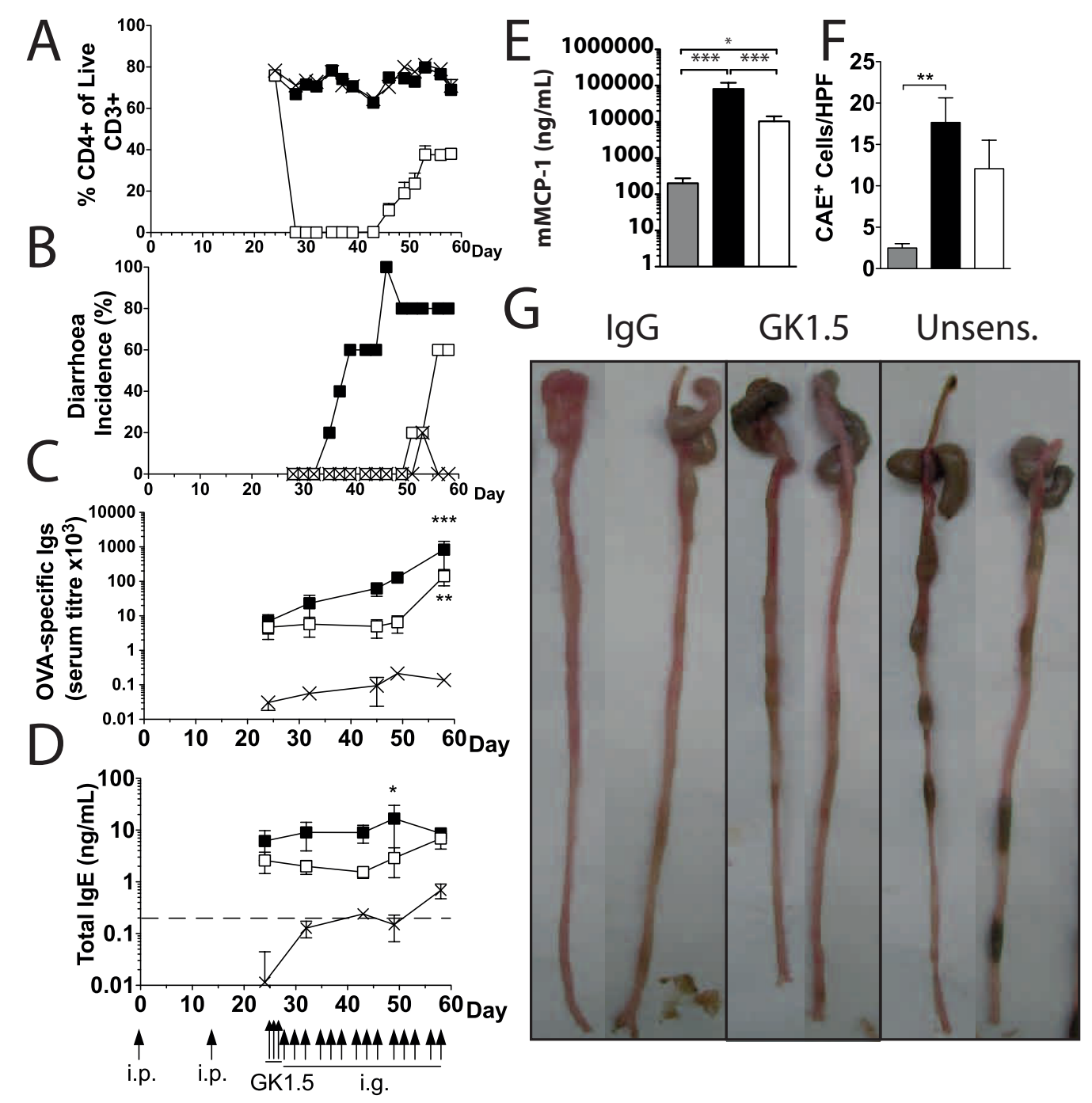

Figure 5.3: Diarrhoea development correlated with the re-emergence of CD4+ T cells. Mice ( $n=5 /$ group) were unsensitized (crosses and grey bars) or sensitized and mock-depleted (black boxes and bars) or i.p. administered $0.5 \mathrm{mg}$ GK1.5 (white boxes and bars) then i.g. challenged with $50 \mathrm{mg}$ OVA as depicted on the x-axis of (D). (A) Live peripheral blood CD3+ cells were examined for CD4 expression by flow cytometry before and after GK1.5 administration, mock-depletion or in unsensitized mice. CD4 depletion was significant versus both other groups at all post-depletion timepoints to $\mathrm{p}<0.0001$. Groups were monitored for $(\mathrm{B})$ diarrhoea development, (C) OVA-specific antibody production, (D) total IgE, and (E) endpoint mMCP-1 release 90-120 minutes after challenge. The horizontal dash in (D) shows the linearity limit of the assay. (F) Ninety minutes after the final challenge mice were euthanized and jejunal section were stained with CAE to quantify mast cells. $(G)$ The picture shows caeca from two representative mock-depleted (IgG), CD4-depleted (GK1.5) or unsensitized (Unsens.) mice after final challenge. Data are shown from a single experiment and were compared (C,D) using Two-way ANOVA with Bonferronis post-test versus unsensitized mice; (E,F) using One-way ANOVA with Tukey's post-test; ${ }^{*} \mathrm{p}<0.05,{ }^{* *} \mathrm{p}<0.01,{ }^{* * *} \mathrm{p}<0.001$. OVA-specific antibody and mMCP-1 data were log-transformed prior to statistical analysis. 


\subsubsection{Segregating CD4+ T cell-dependent mastocytosis and IgE production}

Passive sensitization with IgE enhances mast cell survival, antigen responsiveness and histamine release[30, 45, 293, 314, 453]. As well as producing effector cytokines in the tissue[57], CD4+ T cells control memory B cell activation [288, 407] and are the predominant source of IL-4 required for IgE class switching[275, 287, 289, 362]. It was therefore possible that $\mathrm{CD} 4+\mathrm{T}$ cell depletion prevented antibody titres from reaching sufficient levels to permit disease, and this was the reason the treatment prevented diarrhoea. To determine if systemic antibody production was a limiting factor in intestinal disease development, we increased initial IgE titres by i.p. sensitizing mice multiple times prior to CD4+ T cell depletion (Figure 5.4A). Diarrhoea developed with similar kinetics in twice- and thrice-primed mock-depleted mice (Figure 5.4B). While it was delayed compared to mock-depleted mice, the incidence of diarrhoea also increased at similar rates in twice- and thrice-primed CD4-depleted mice (Figure 5.4B). mMCP1 production was also impaired in CD4-depleted mice (Figure 5.4C), and only mockdepleted mice had statistically elevated jejunal mast cell numbers of PBS-fed mice (Figure 5.4D). However, there was a clear trend indicating mastocytosis increased in CD4-depleted mice by the end of the experiment (Figure 5.4D). In this experiment, we observed that CD4+ T cell depletion was maintained in both twice- and thriceprimed mice for the first five challenges (Figure 5.4E), but as they returned in thriceprimed mice, Total IgE levels began to increase (Figure 5.4F). However, critical to our results, after five oral challenges, CD4-depleted thrice-primed mice maintained similar Total IgE and OVA-specific IgE levels to mock-depleted twice-primed mice, but were protected from disease (Figure $5.4 \mathrm{~B}-\mathrm{D}$ ). By the $5^{\text {th }}$ challenge $50 \%$ of twice-primed mock-depleted mice had developed diarrhoea (Figure 5.4B). Thus, antibody titres were sufficiently high in CD4+ T cell-depleted mice to permit disease, but disease did not develop, indicating that CD4+ T cells promoted jejunal mastocytosis in a manner that was independent of their involvement in systemic antibody production. 
A

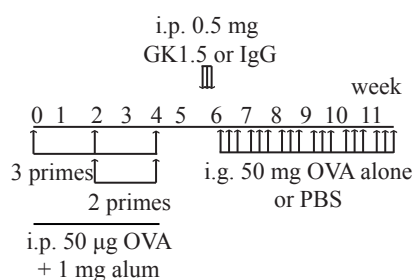

C

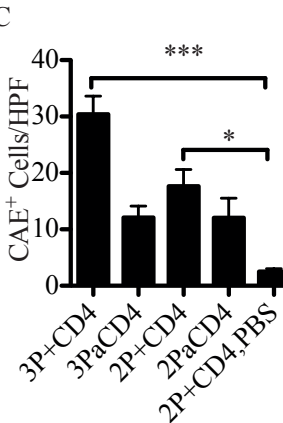

$\mathrm{D}$

B
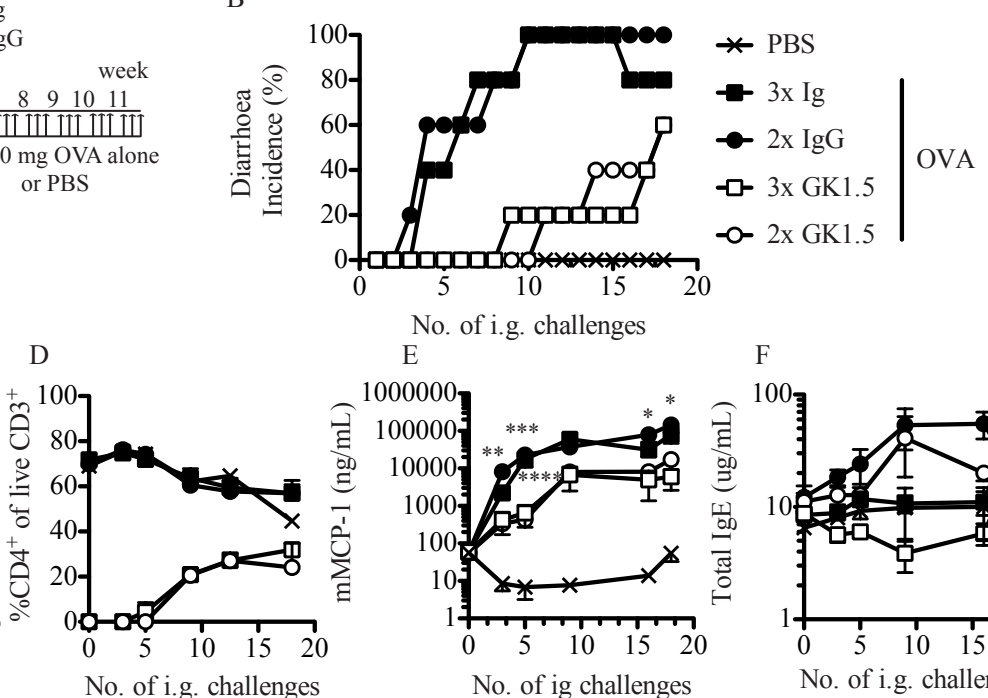

No. of ig challenges

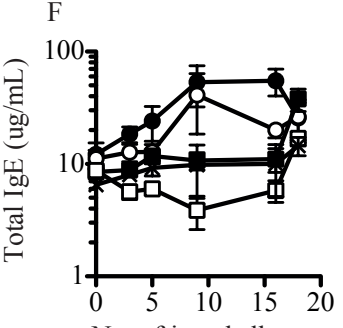

No. of i.g. challenges
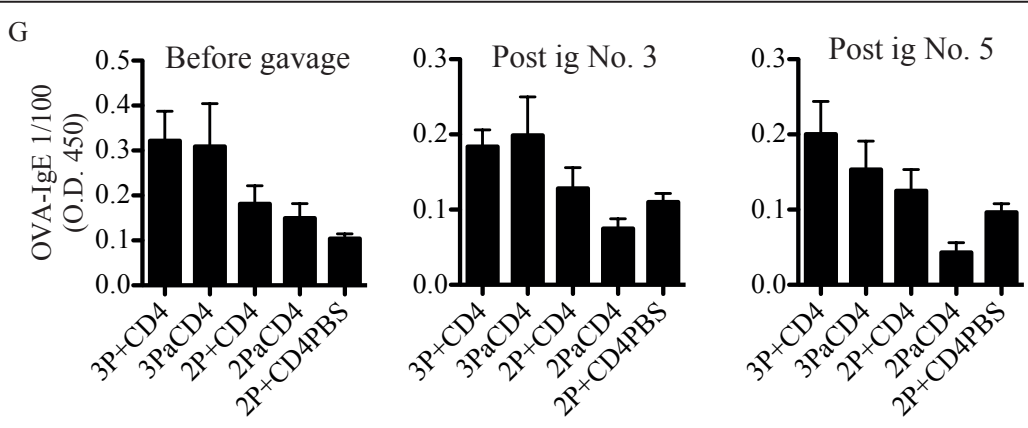

Figure 5.4: High-titre IgE can only confer disease susceptiblity in the presence of $\mathbf{C D} 4+\mathbf{T}$ cells. Mice ( $\mathrm{n}=5 /$ group) received two $(2 \mathrm{P}$; circles) or three (3P; squares) i.p. injections of OVA plus alum adjuvant as indicated in (A), were administered GK1.5 (aCD4; white symbols) to deplete CD4+ T cells or were mock-depleted with polyclonal Rat IgG (+CD4; black symbols) and from week six were i.g. challenged with OVA or PBS (PBS). Twice-primed mice that were fed PBS served as controls (PBS; crosses). The full protocol is shown in (A). (B) Diarrhoea was monitored after each challenge. (C) mMCP-1 was determined by ELISA. (D) CAE+ (Mast) cells were examined in jejunal sections of mice after the last challenge. (E) Mice were periodically bled to monitor peripheral CD4 T cell blood content. (F) Total and $(\mathrm{G})$ OVA specific-IgE were monitored during the experiment. Data are represented as group mean \pm SEM. Data are shown from one experiment and were analysed (C) using Two-way ANOVA, OVA-challenged non-depleted mice versus thrice-primed CD4-depleted mice, or (D) using One-way ANOVA with Tukey's post-test. $\mathrm{p}<0.001$. ${ }^{*} \mathrm{p}<0.05,{ }^{* *} \mathrm{p}<0.01,{ }^{* * *} \mathrm{p}<0.001,{ }^{* * *} \mathrm{p}<0.001$. Similar observations were made in a second experiment comparing only thrice-primed GK1.5-treated mice and twiceprimed mock-depleted groups. ( $\mathrm{n}=5 /$ group). 


\subsubsection{Examining the role of $\mathrm{CD} 4+\mathrm{T}$ cells in disease mainte- nance}

$\mathrm{CD} 4+\mathrm{T}$ cells were required for diarrhoea to be exhibited. This lead us to ask if CD4+ $\mathrm{T}$ cells maintained local responses after oral potentiation. Next we investigated how $\mathrm{CD} 4+\mathrm{T}$ cell depletion affected established disease. We orally challenged mice until they presented with diarrhoea, then depleted them of CD4+ T cells (Figure 5.5). Administration of GK1.5 depleted CD4 $+\mathrm{T}$ cells to $<1$ per 4000 peripheral blood CD3+ $\mathrm{T}$ cells, whereas mock-depleted animals maintained a proportionally normal $\mathrm{T}$ cell pool (Figure 5.5A). This level of depletion was maintained by GK1.5 injection every four days and was also observed in all peripheral tissues, including the intestine at the experimental end-point (Figure 5.5B and 5.5C). Reciprocal increases in the CD8+ proportion were observed in all tissues (Appendices Figure 8.4). Prior to depletion, both OVA-challenged groups had similar mMCP-1 serum titres (Figure $5.5 \mathrm{E}$, left), but after CD4+ T cell depletion, challenge-induced mMCP-1 release was decreased (Figure 5.5E, right). This correlated with fewer mast cells in jejunal tissues of CD4-depleted mice at the experimental end-point (Figure 5.5F), and lower levels of Total and OVA-specific IgE than mock-depleted controls following the depletion (Figure 5.5G). Despite reduction in Th2-associated parameters, diarrhoea ensued in the majority of CD4-depleted mice for at least seven further challenges (7/10 mice; Figure 5.5D). We also observed that while Total and OVA-specific IgG2a titres were unaltered (Figure 5.6A and 5.6B), CD4-depleted mice had lower Total and OVAspecific IgG1 titres than mock-depleted mice (Figure 5.6C and 5.6D), and fewer splenic and bone marrow OVA-IgG1 antibody secreting cells (ASCs) (Figure 5.6E and 5.6F). Total or OVA-specific IgG2a titres were unaltered (Figure 5.6A and 5.6D). These data suggest that while CD4+ T cells maintained underlying disease susceptibility, once instated, the response could be triggered for long periods in their absence. 

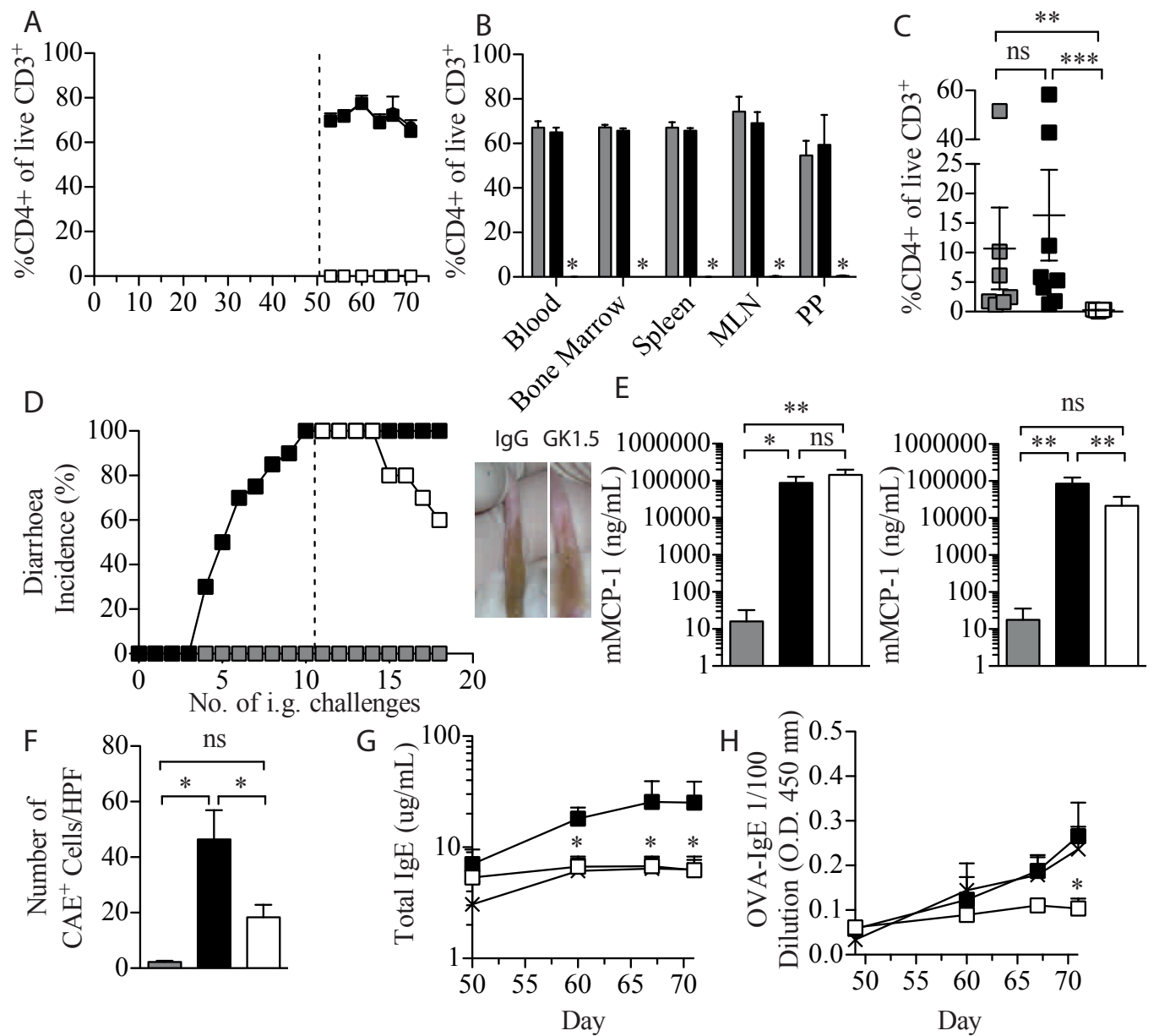

Figure 5.5: Orally primed mice exhibited diarrhoea even in the absence of CD4+ T cells. Mice were sensitized and orally challenged with OVA ten times, at which point all challenged mice had developed diarrhoea. One group was then administered GK1.5 to deplete CD4+ T cells and the depletion was maintained throughout the experiment. Sensitized mice that were fed with PBS alone served as baseline controls. Black boxes and bars represent non-depleted mice, white boxes and bars represent CD4-depleted mice, and grey bars and black crosses represent baseline controls. The experimental regimen is outlined on the $\mathrm{x}$-axis in $(\mathrm{A})$. The dashed lines in (A) and (D) represent the point at which CD4+ T cells were depleted. (A) Live CD3+ peripheral blood cells were monitored for CD4 expression by flow cytometry. (B) After the final i.g. feed, mice were euthanized and blood, bone marrow, spleen, mesenteric lymph nodes (MLN), peyers patches (PP) and the jejunal lamina propria (LPL) were examined by flow cytometry for the proportion of live CD45+CD3+ T cells that expressed CD4. (C) The intraepithelial compartment was assessed for CD4+ $\mathrm{T}$ cells as in (B). (D) Diarrhoea was monitored in PBS-fed or OVA-fed mice administered GK1.5 or Rat IgG at the dashed line. The photo shows diarrhoea in mice from the specified groups after challenge seventeen. (E) Serum mMCP-1 was measured following challenge ten (left; prior to depletion) and fourteen (right; after depletion). (F) At the experimental endpoint, jejunal sections were examined for CAE + cells. (G) Serum Total IgE and (H) OVA-specific IgE was measured by ELISA. Data in (B-D) are combined from two experiments ( $\mathrm{n}=8$ PBS-fed; $\mathrm{n}=9$ Mock-depleted; $\mathrm{n}=10$ CD4-depleted) mice and (A, E-H) are shown from one of the two similar experiments with $(n=4-5)$ mice/group, because the exact timings of the analyses were not identical. Data were compared (A, B, G, H) using Two-way ANOVA with Bonferroni's post-test, (C) using Kruskall-Wallis with Dunn's post-test, or (E, F) using One-way ANOVA with Tukey's post-test; ${ }^{*} \mathrm{p}<0.05,{ }^{* *} \mathrm{p}<0.01,{ }^{* * *} \mathrm{p}<0.001,{ }^{* * * *} \mathrm{p}<0.0001$. 


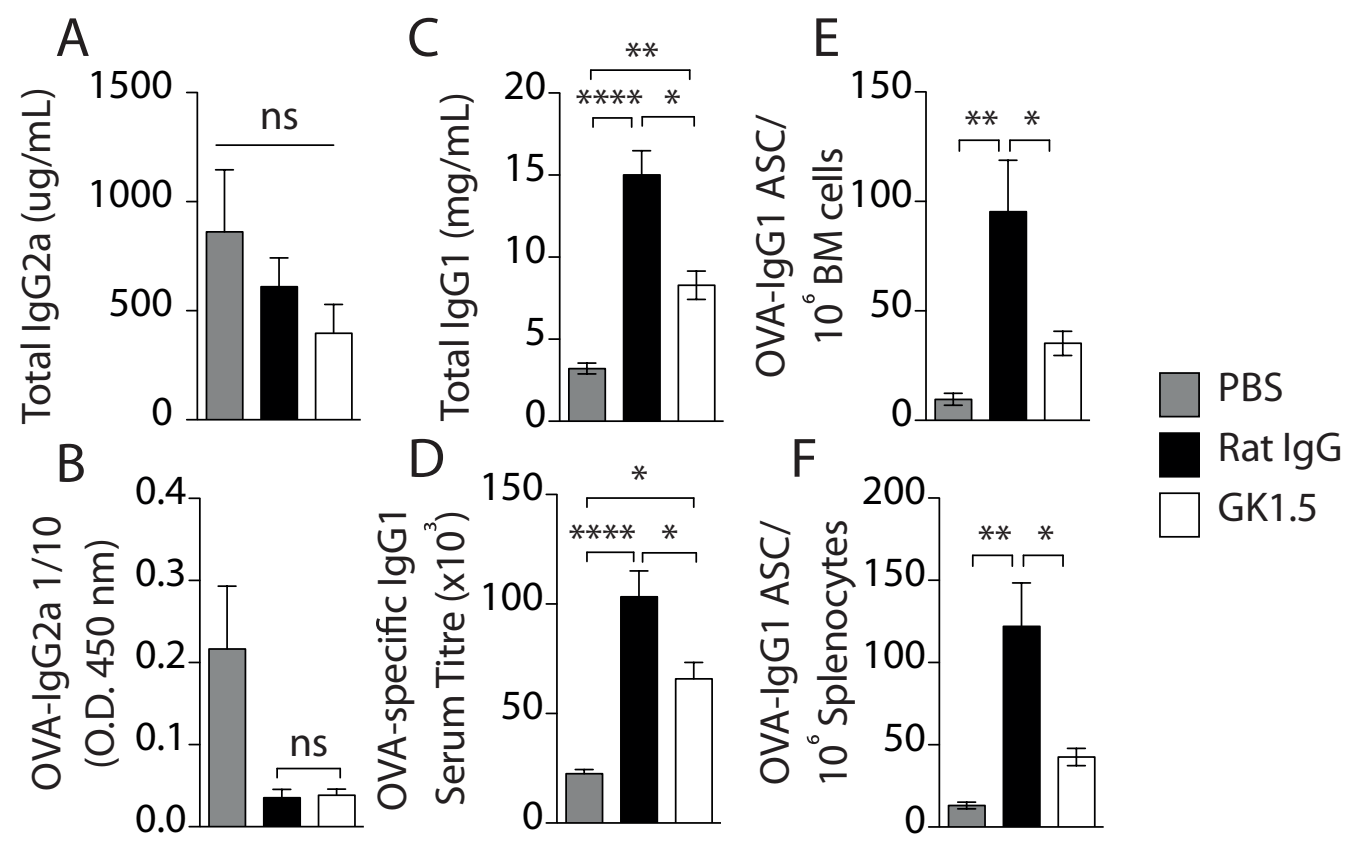

Figure 5.6: CD4+ T cell depletion diminished the Th2-associated antibody response. OVA-sensitized mice fed with PBS (grey bars), or administered GK1.5 (white bars) or Rat IgG (black bars) after ten oral OVA feeds (shown in Figure 5.5) were bled at the experimental end-point. Sera were analysed for (A) Total IgG2a, (B) OVA-specific IgG2a, (C) Total IgG1, (D) OVA-specific IgG1. (E,F) Anti-OVA-IgG1 antibody secreting cells (ASCs) were quantitated in the bone marrow or spleens. Data represents mean \pm SEM of $(n=3-5)$ mice from one of two similar experiments. Data were analysed using One-way ANOVA with Tukey's post-test; ${ }^{*} \mathrm{p}<0.05,{ }^{*} \mathrm{p}<0.01$, $* * * * \mathrm{p}<0.0001$. 


\subsubsection{The role of CD4+ $\mathrm{T}$ cells in systemic responses after oral potentiation}

Oral potentiation has been shown to increase the severity of systemic anaphylaxis induced by i.v. allergen challenge[37]. In a final experiment using GK1.5, we examined the development of systemic anaphylaxis in mice depleted of CD4+ T cells after oral potentiation.

Similar to the previous experiment, mice were challenged until diarrhoea presented, and were depleted of CD4 $\mathrm{T}$ cells for the remainder of the experiment. Again, diarrhoea ensued in the absence of CD4+ $\mathrm{T}$ cells (Figure 5.7A). In contrast to the previous report[37], when mice were administered $100 \mu \mathrm{g}$ OVA i.v. 48 hours after oral challenge, i.p.-sensitized mice demonstrated equivalent hypothermia severity, irrespective of whether they were fed OVA or not (Figure 5.7B). Unexpectedly, evidence of anaphylactic diarrhoea appeared only in mice that had been orally primed, because 10-15 minutes after i.v. challenge, only mice that had previously received oral OVA challenges had matted fur around the anus (Figure 5.7C). This occurred in both CD4depleted and mock-depleted groups(Figure 5.7C), and was not observed in these mice when temperatures were monitored prior to i.v.-challenge. Antibody levels were similar to those reported in the previous experiment (Figure 5.7D-G). These data suggest that oral potentiation may alter the physiology of the gut, or the gut content, to permit intestinal involvement in the i.v.-induced allergic response. 


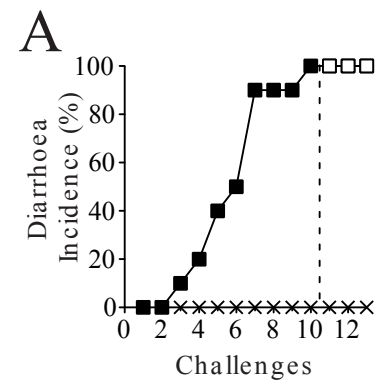

$\mathrm{C}$

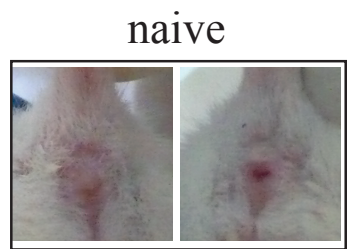

OVA/OVA mock dep

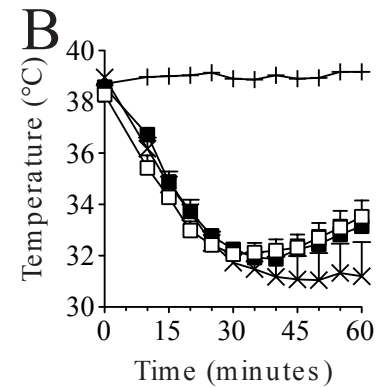

Unprimed

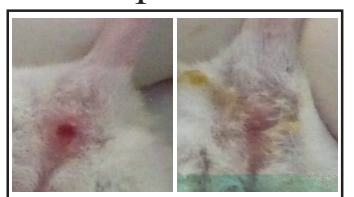

OVA/OVA CD4 dep
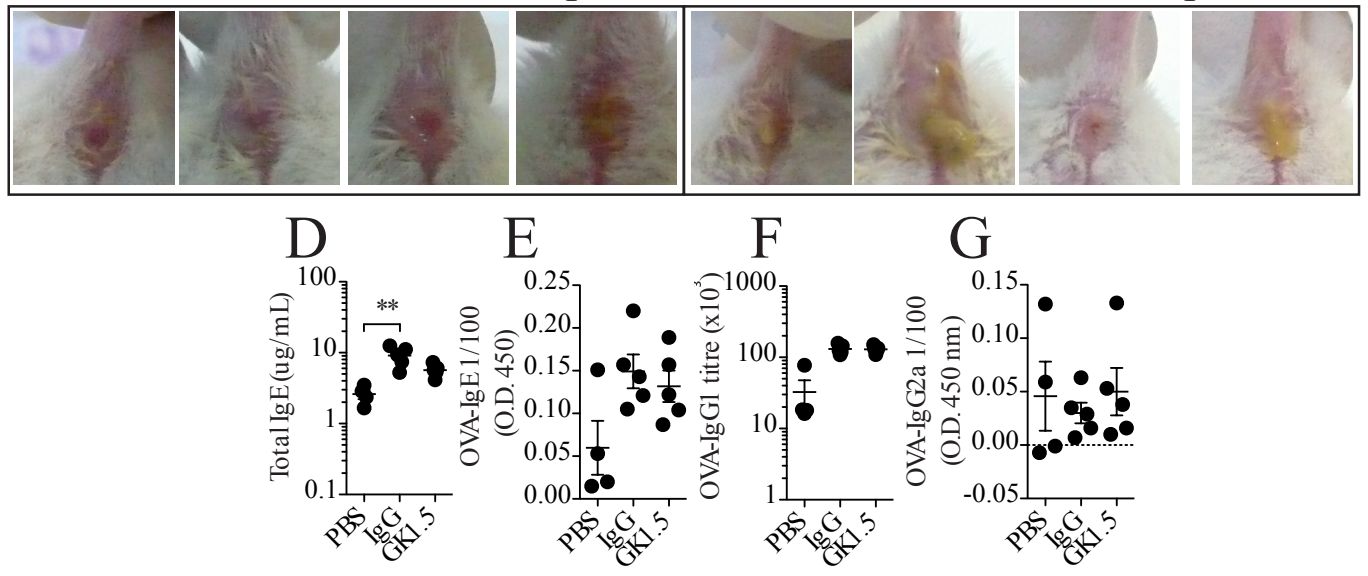

Figure 5.7: Orally-primed mice developed i.v.-induced anaphylaxis with or without CD4 $+\mathbf{T}$ cells. (A) Mice were sensitized and fed OVA (boxes) or PBS (crosses). After ten challenges OVA-fed mice were administered three $0.5 \mathrm{mg}$ doses of GK1.5 (white boxes) or Rat IgG (black boxes), 24 hours apart, after the tenth challenge indicated by the dashed line in (A). (B) Forty-eight hours after the final oral challenge mice were administered $100 \mu \mathrm{g}$ OVA i.v. and monitored for temperature changes. Completely naive mice (plus symbols) treated with $100 \mu \mathrm{g}$ OVA i.v. served as unsensitized controls. (C) mice were photographed 10-25 minutes after i.v. challenge. Bottoms were normal prior to i.v. challenge. Mice were bled 24 hours after the final oral challenge, prior to i.v. challenge and sera were analysed by ELISA for (D) Total IgE, (E) OVA-specific IgE, (F) OVA-specific IgG1, (G) OVA-specific IgG2a. Data represent group mean $\pm S E M ; n=3$ (naive), $n=4$ (PBS), or $n=5$ mice/group from one experiment. Data were compared using One-way ANOVA with Tukey's post-test; ${ }^{* *} \mathrm{p}<0.01$. 


\subsubsection{Influence of CD4+ $\mathrm{T}$ cells on plasma volume loss}

Similar to studies of others[57], we demonstrated that diarrhoea development in orallyprimed mice correlated with an increase in blood haematocrit (Figure 5.8A). This provides an additional method by which to assess the development of an intestinal allergic response. In our first experiment in this chapter, mock-depleted mice developed diarrhoea and CD4-depleted mice were protected from diarrhea (Figure 5.1). Simiarly, we observed elevated blood haematocrit in mock-depleted mice with active diarrhoea (Figure 5.8B), but not in the CD4-depleted mice (Figure 5.8B and 5.8C), whereas mice that had developed diarrhoea prior to their CD4-depletion showed similar increases in their haematocrit to mock-depleted mice (figure 5.8D). While naive mice were not analysed, the PBS-fed groups and prior-to-challenge groups indicate that there was indeed a change in blood plasma volume associated with the diarrhea. Therefore, mice that presented with diarrhoea lost fluid volume in both the presence and the absence of CD4+ T cells.

Taken together, these data suggest that CD4+ T cells were required for oral allergeninduced intestinal mastocytosis that permitted allergic diarrhoea. Once established, however, intestinal allergy persisted for long periods in the absence of CD4+ T cells. Further mice remained susceptible to systemic i.v.-induced anaphylaxis in the absence of CD4+ T cells with or without prior oral potentiation. 


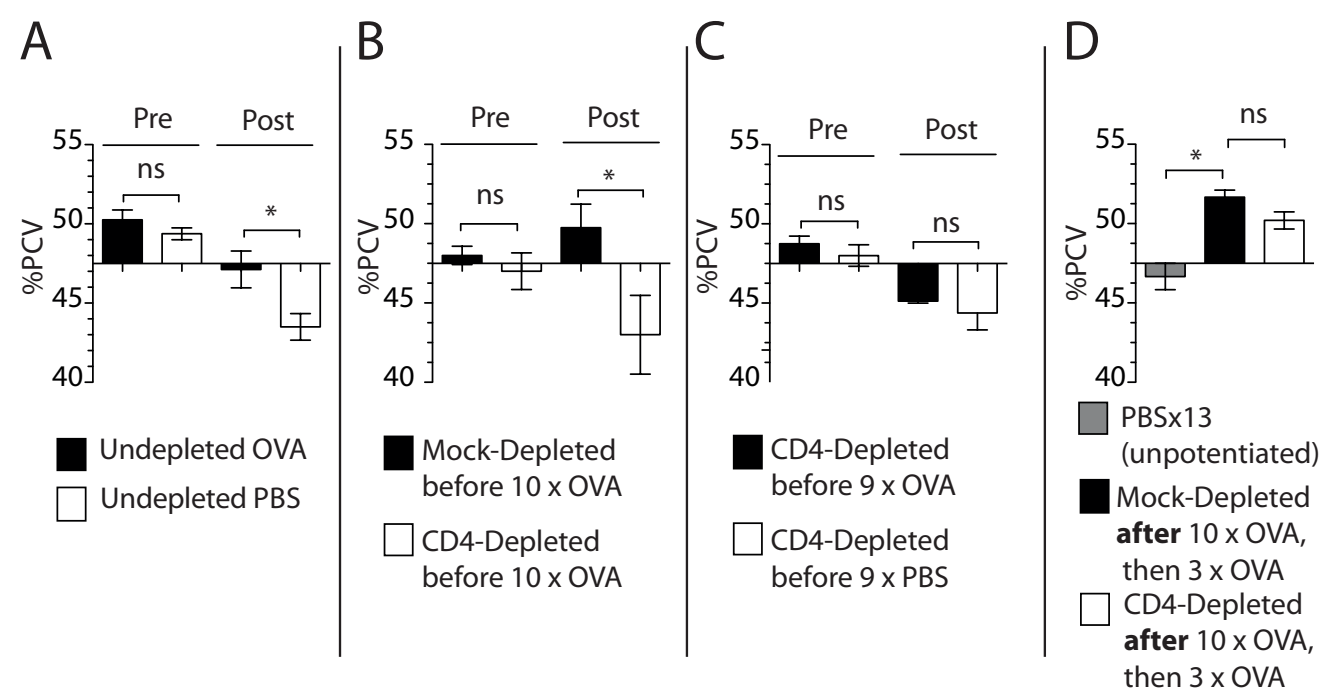

Figure 5.8: Haematocrit increases in intestinally anaphylactic mice with or without CD4+ T cells. (A) OVA-sensitized mice were bled before and after the ninth oral gavage with OVA or PBS and their haematocrit was assessed. All OVA-fed mice had active diarrhoea at the time of bleeding. (B) Mice shown in (Figure 5.1) were bled prior to and following the ninth i.g. challenge for haematocrit. (C) Mice depleted of CD4+ T cells prior to being fed OVA or PBS with depletions maintained thereafter (same mice as in Figure 5.2) were bled for haematocrit prior to and following the ninth i.g. challenge. (D) After the thirteenth i.g. challenge, mice shown in Figure 5.7 were bled for haematocrit. data in each (A-D) is from a single experiment ( $n=3-5 /$ group). Data were analysed using (A-C) Mann-Whitney U-test or (D) using Kruskal-wallis with Dunn's post-test; ${ }^{*} \mathrm{p}<0.05$. 


\subsubsection{The cytokines that influence allergic mastocytosis}

In addition to supporting orally induced IgE production, CD4+ $\mathrm{T}$ cells promoted mastocytosis in an additional process. In addition to IL-4 and IL-9, IL-3 is implicated in Th2-type intestinal mast cell responses. Whether oral allergen-induce mastocytosis is dependent on IL-3 is unknown.

As a start point, because IL-3 is associated with a Th2-type intestinal response[315], and allergic diarrhoea is associated with a Th2 cell profile[37, 57, 58], we were interested to determine if Th2 conditioning would trigger IL-3 production from T cells. We examined the production of IL-3 by anti-CD3 stimulated splenocytes cultured under Th0, Th1 and Th2 conditions. When total spleen cells were added to culture medium containing IL-4 (Th2 conditioning medium) and stimulated with anti-CD3 for three days, splenocyte IL-3 production reached approximately $5 \mathrm{ng} / \mathrm{mL}$, whereas under Th0 or Th1 conditions levels were lower, reaching 1 and $0.5 \mathrm{ng} / \mathrm{mL}$ respectively (Figure 5.9A). Similarly, when total splenocytes were washed and transferred to uncoated or new anti-CD3 plates without additional IL-4, the amount of IL-3 produced in preconditioned Th2 spleen cell cultures was 4-5-fold higher than under Th1 conditions (Figure 5.9B). When parallel cultures were stained for intracellular cytokine, $10 \%$ of CD4+ T cells from the Th2 splenocyte cultures stained positive for IL-3 after the six hour culture period, whereas only $2 \%$ of Th1 cultured cells contained intracellular IL-3 (Figure 5.9C). Therefore, we determined that Th2 conditions promoted IL-3 production by $\mathrm{CD} 4+\mathrm{T}$ cells. 

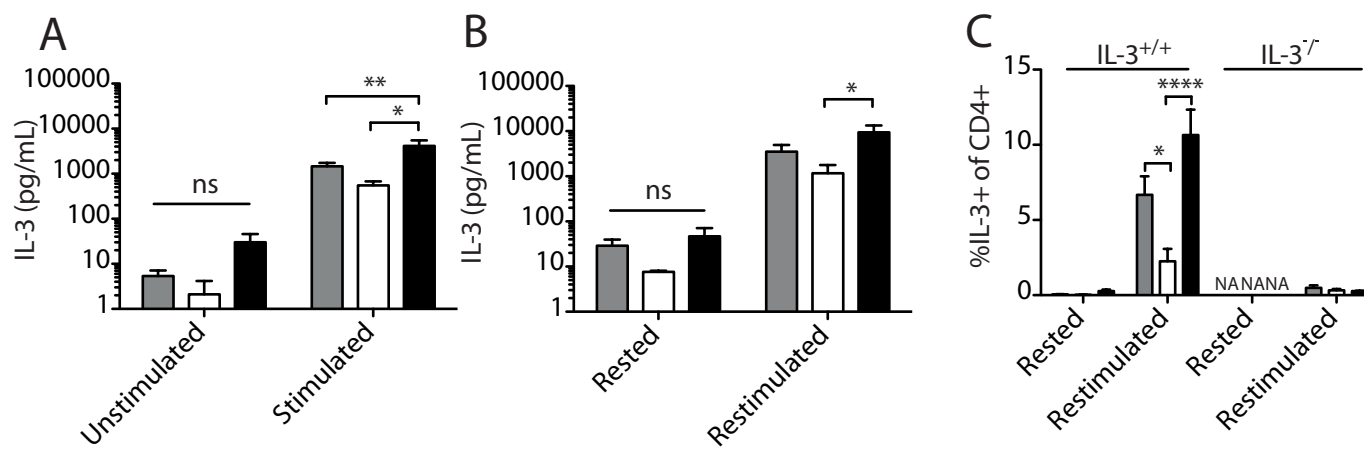

Figure 5.9: Th2 cells produced more IL-3 than Th1 cells. (A) Splenocytes were cultured for three days on uncoated (Unstimulated) or anti-CD3 coated (Stimulated) plates under Th0 (grey) Th1 (white) or Th2 (black) conditions and supernatants were analysed for IL-3 by ELISA. (B) After three days stimulation on anti-CD3 under Th0 (grey) Th1 (white) or Th2 (black) conditions splenocytes cultured from individual mice were washed, transferred to restimulation medium and replated in uncoated (Rested) or anti-CD3-coated wells (Restimulated) for 6 hours and supernatants were analysed for IL-3 by ELISA. (C) Side-by-side cultures of cells in (B) had monensin added after 4 hours of culture and were stained for intracellular IL-3 two hours later. Data are represented by mean \pm SEM: $(A)$ individually cultured spleens $(n=4)$ were collated from two experiments; data in (B and C) individually stimulated IL-3+/+ spleens $(n=7)$ collated from three separate experiments. In one experiment IL-3-/mouse spleens $(n=2)$ were included to test antibody specificity, and in (B) one spleen was not analysed under Th0 conditions due to a low cell yield. NA=Not assessed. For restimulation, 500000 cells from activated wells were replated from each condition and restimulated in medium containing IL-2 and anti-CD28. Data were analysed using Two-way ANOVA with Bonferroni's post-test; ${ }^{*} \mathrm{p}<0.05,{ }^{*} \mathrm{p}<0.01,{ }^{*} * * * \mathrm{p}<0.0001$. 
IL-3 production is known to promote the development of mast cells, but also contributes to basophil development from bone marrow cells. Recent evidence demonstrates that Thymic Stromal Lymphopoeitin (TSLP) can also promote the development of basophils from bone marrow cells[429], and TSLPR-deficient mice show reduced disease incidence in this model[132]. This led us to investigate a potential and unknown role for TSLP in mast cell generation. However, while we showed that TSLP caused a significant increase in the proportion of basophils induced to produce IL-4 (as observed previously[429]) in G4/+ mice (Figure 5.10E and 5.10F), only IL-3containing cultures caused mast cells to develop (Figure 5.10C and 5.10F). Mast cells are the key effector cell in the ipig model of allergic diarrhoea[37], indicating that while TSLP may be involved in the allergic process[132], IL-3, rather than TSLP, promoted mast cell development from bone marrow cells. 

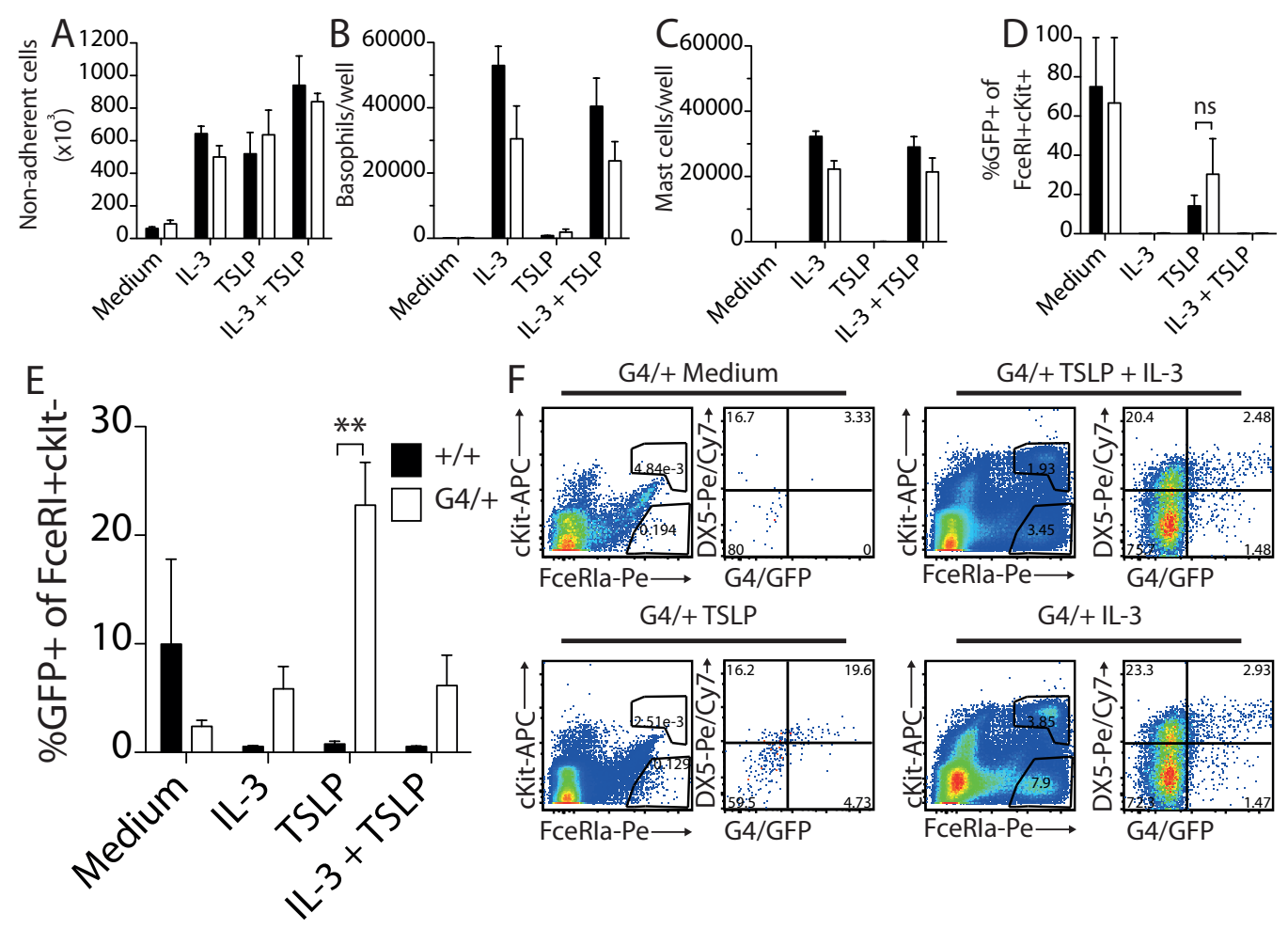

Figure 5.10: IL-3 but not TSLP caused mast cells to develop in vitro. Bone marrow was isolated from $(n=3)+/+$ (black bars) or $(n=3) \mathrm{G} 4 /+$ (white bars) mice cells were cultured from each individual mouse for five days in the presence of 10 $\mathrm{ng} / \mathrm{mL}$ IL-3, $1 \mu \mathrm{g} / \mathrm{mL}$ TSLP, both IL-3 and TSLP or medium alone. (A) Total cells were counted by trypan blue exclusion and numbers were used to back-calculate numbers of (B) basophils and (C) mast cells ascertained by flow cytometry. (D) The proportion of mast cells that expressed GFP and (E) the proportion of basophils that expressed GFP were assessed in each culture. (F) Left plots show the $\mathrm{cKit}^{\text {hi }}$ FceRI+ population used to define mast cells and the cKit ${ }^{\text {low }} \mathrm{FceRI}+$ cells that were further examined for DX5 and GFP expression (basophils; right plots). Data are shown from one experiment. Data were compared using Two-way ANOVA with Bonferroni's post-test; ${ }^{* *} \mathrm{p}<0.01+/+$ versus $\mathrm{G} 4 /+$. 


\section{Allergic mastocytosis and diarrhoea were impaired in IL-3- deficient mice.}

To determine the potential involvement of IL-3 in CD4+ T cell-, IgE- and mast celldependent allergic intestinal anaphylaxis, we examined the development of allergic diarrhoea in IL-3-/- mice. When IL-3-/- mice were sensitized i.p. to OVA/Alum, Total and OVA-specific IgE were detected in serum (Figure 5.11B and C). Similar to wild-type mice, their Total and OVA-specific IgE levels increased following oral gavage challenge (Figure 5.11B and C), albeit to lower absolute levels than IL-3+/ + mice. IL3-/- mice were protected from allergic diarrhoea (Figure 5.11A and G) and at the experimental end-point their intestines still contained numerous pellets (Figure 5.11F). Oral OVA challenge did not increase haematocrit in IL-3-/- mice, nor in PBS-fed IL-3+/+ mice; haematocrit in both groups remained close to $47.5 \%$, whereas haematocrit was increased to approximately $60 \%$ of blood volume in diarrhoetic OVA-fed IL-3+/+ mice (Figure 5.11D). When we examined the mast cell response, mMCP-1 levels were lower in IL-3-/- mice (Figure 5.11E), and this correlated with fewer jejunal mast cells in OVA-fed IL-3-/- mice than IL-3+/+ mice (Figure 5.11F). Differences in jejunal mast cell frequencies at baseline were not statistically distinguishable, in line with no requirement for IL-3 for basal mastocytosis as reported by other groups[309]. In preliminary examinations we also observed similar Th2 cytokine production in spleen cell preparations from OVA-sensitized IL-3+/+ and IL-3-/- mice (Appendix Figure 8.5A - D). Further, similar to an earlier study[455], we found that both IL$3+/+$ and IL-3-/- mice were susceptible to i.v. allergen-induced systemic anaphylaxis (Figure 5.12B). This occurred even when IL-3-/- mice were fed with OVA (Figure $5.12 \mathrm{~B})$. Therefore, these data suggest that IL-3 was required for the generation of an intestinally-localised mast cell-dependent allergic response. 


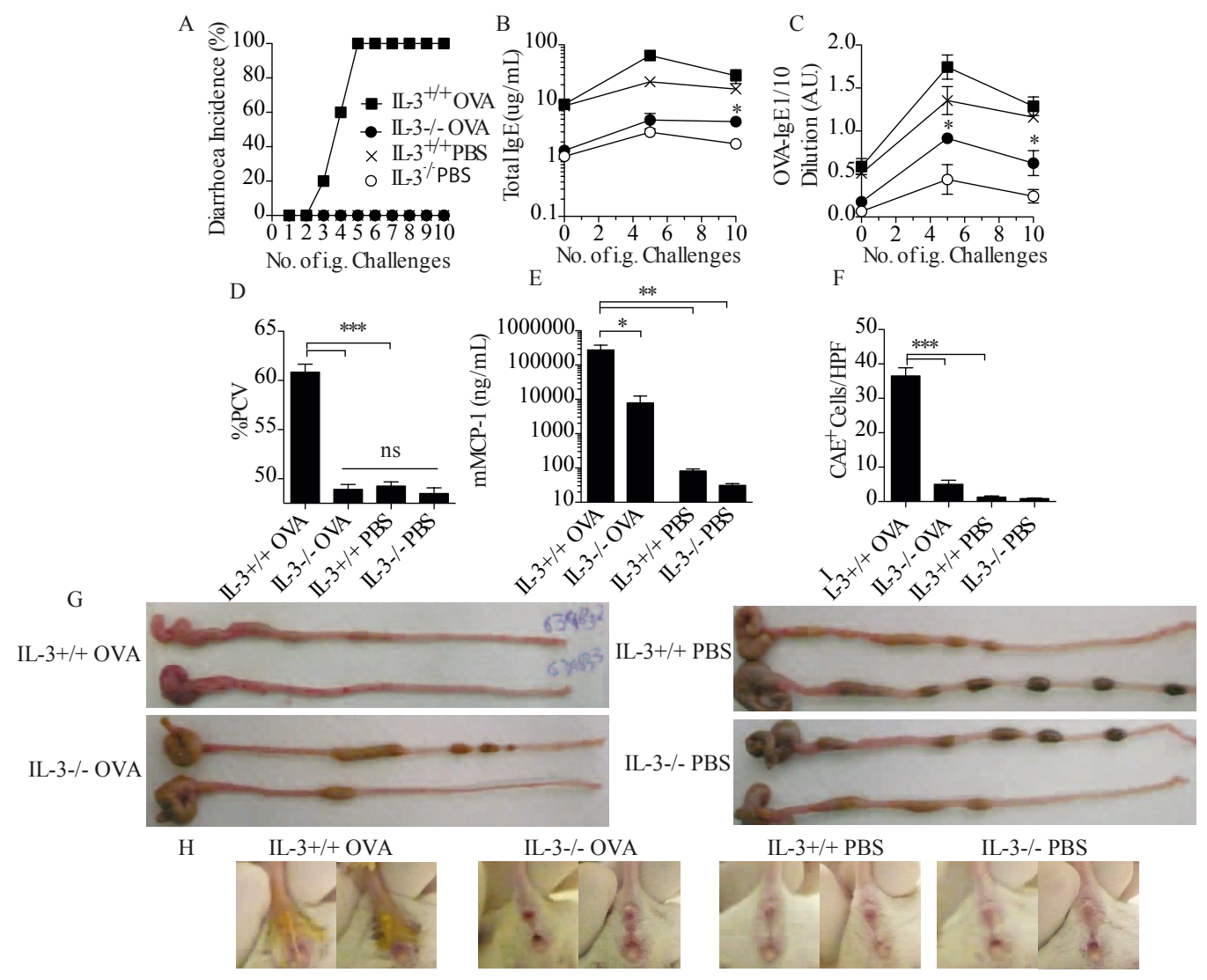

Figure 5.11: IL-3-/- mice were protected from allergic diarrhoea. (A) OVAsensitized IL-3-/- and IL-3+/+ mice were i.g. fed with OVA or PBS and monitored for diarrhoea development. (B) Total IgE and (C) OVA-specific IgE were measured by ELISA. (D) Haematocrit (\%PCV) was measured 50 minutes after challenge 9 and (E) mMCP-1 release was measured after challenge ten. (F) Jejunal sections from mice challenged ten times were stained for CAE+ (mast) cells. Photographs were taken of $(\mathrm{G})$ colons 90 minutes after challenge ten or $(\mathrm{H})$ bottoms 60 minutes after challenge nine. Genotypes are specified at the top of the page. (A) Diarrhoea curves are shown from one of five similar experiments each with $(n=4-5)$ mice/group. (D) \%PCV was measured once and all other data were analysed in three of the experiments with similar results in each case. Data are represented by mean \pm SEM. Data were analysed $(\mathrm{B}, \mathrm{C})$ using Two-way ANOVA with Bonferroni's post-test, OVA-fed IL-3-/- vs. PBSfed IL-3-/- mice or (D-F) using One-way ANOVA with Tukey's post-test; ${ }^{*} \mathrm{p}<0.05$, $* * \mathrm{p}<0.01, * * * \mathrm{p}<0.001$ 

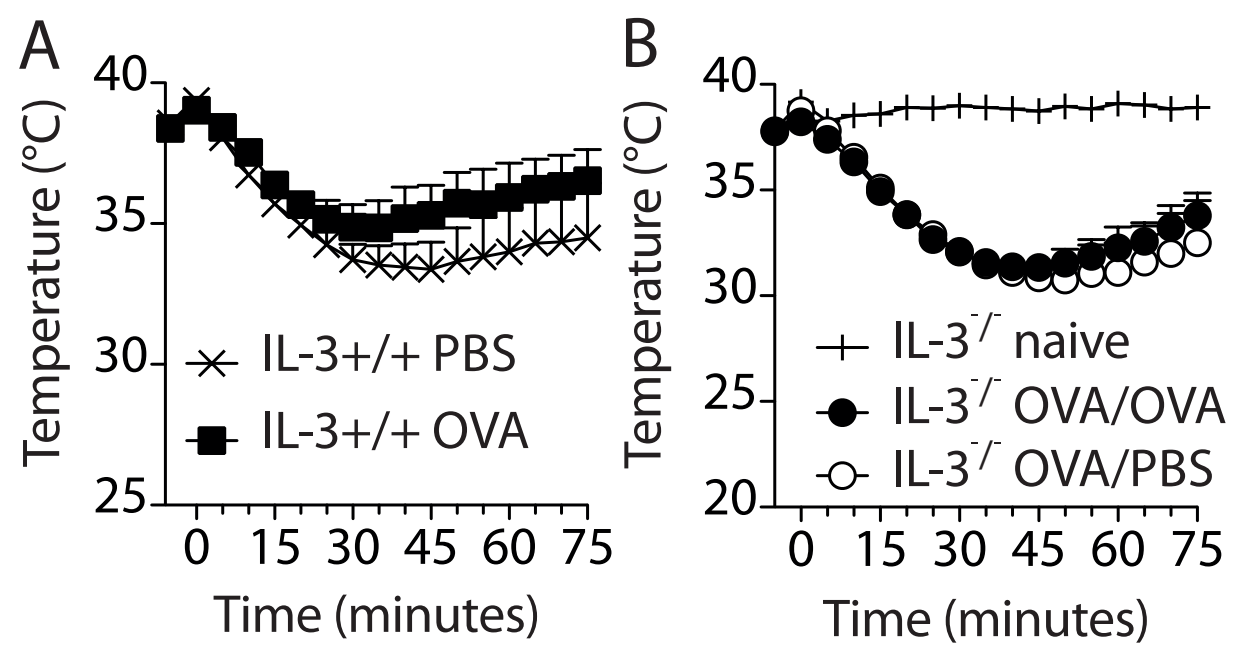

Figure 5.12: IL-3-/- mice were susceptible to i.v.-induced systemic anaphylaxis. (A) Wild-type and (B) IL-3-/- mice were sensitized to ovalbumin and challenged six times with PBS or OVA and then received $100 \mu \mathrm{g}$ OVA i.v. and temperature change was monitored. Data are represented by mean \pm SEM from a single independent experiment ( $\mathrm{n}=2-3$ mice/group). 


\subsubsection{The relative influence of IL-3 and IL-9 on allergic mas- tocytosis}

Mice carrying a single allele of the $i F A B P p$-IL-9 transgene $(\mathrm{Tg}+/+)$ over-express IL9 locally in the jejunum[57]. This increases basal mast cell activity and predisposes mice to allergic diarrhoea and is also associated with higher oral allergen-induced mast cell activity compared with non-transgenic mice[57]. We next investigated whether over-expression of IL-9 could amplify jejunal mastocytosis in the absence of IL-3. In these experiments we compared $\mathrm{Tg}+/+$ mice to IL-3-deficient mice carrying the transgene ( $\mathrm{Tg}$-/-), and sib-matched non-transgenic IL-3-deficient mice (nTg-/-). nTg/- is used to distinguish between IL-3-/- mice and IL-3-deficient mice sib-matched to $\mathrm{Tg}$-/- mice.

When sera were analysed from naive $\mathrm{Tg}+/+$ mice, $\mathrm{Tg}$ - $/-$ and $\mathrm{nTg}$ - $/$ - mice, mice carrying the transgene had 4 - to 5-fold higher serum mMCP-1 levels than nTg-/mice (Figure 5.13A). Similarly, when jejuni were sectioned and examined for CAE+ mast cells, $\mathrm{CAE}+$ cells were on average, twice as frequent in naive $\mathrm{Tg}$-/- mice than naive $\mathrm{nTg}-/-$ mice(Figure 5.13B). CAE+ cells were present at similar levels in naive $\mathrm{Tg}$-/- mice as in naive $\mathrm{Tg}+/+$ mice (Figure 5.13B). Therefore, in the absence of IL-3, over-expression of IL-9 in the jejunum increased basal jejunal mast cell activity. 

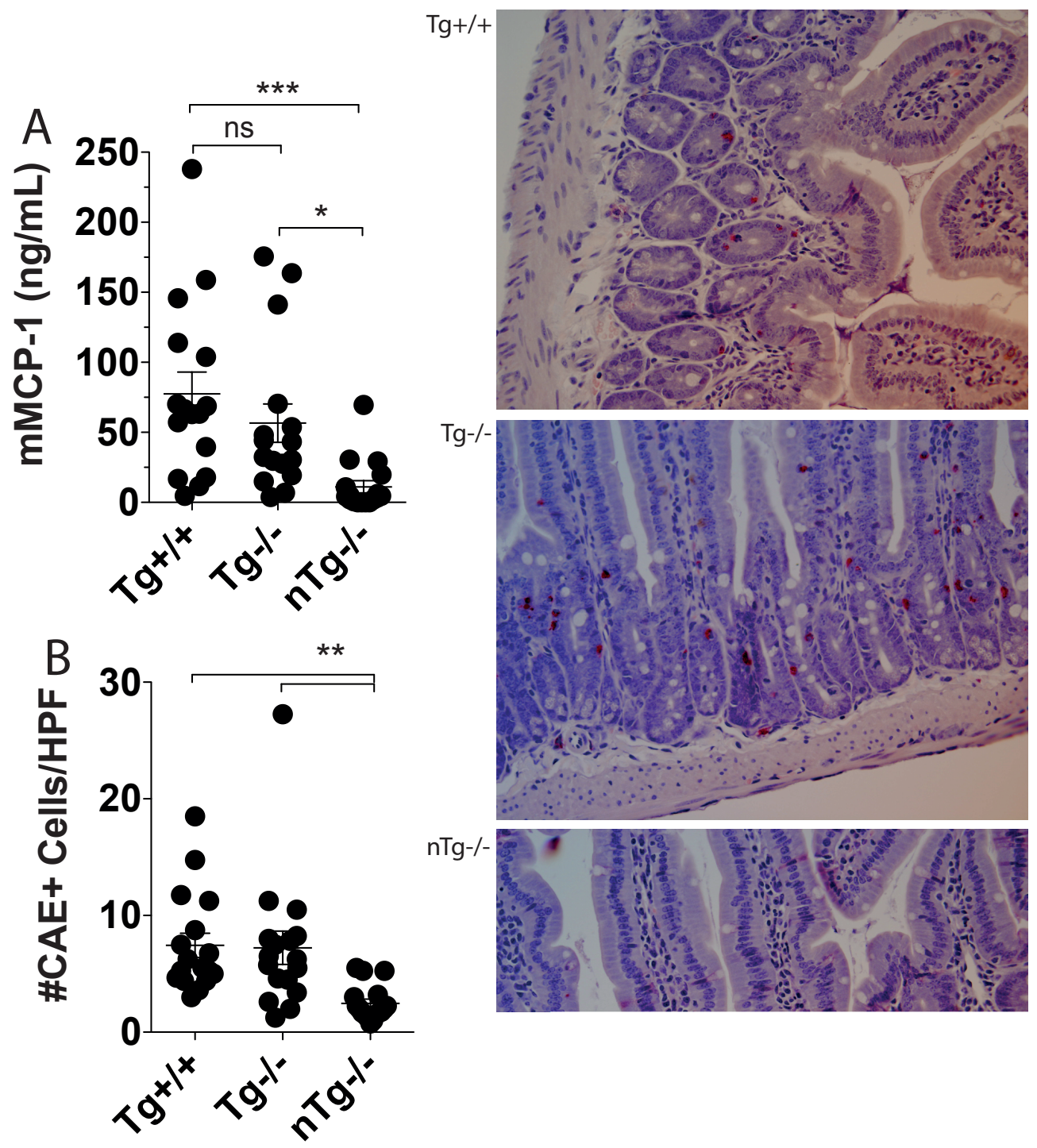

$\mathrm{nTg}-/-$

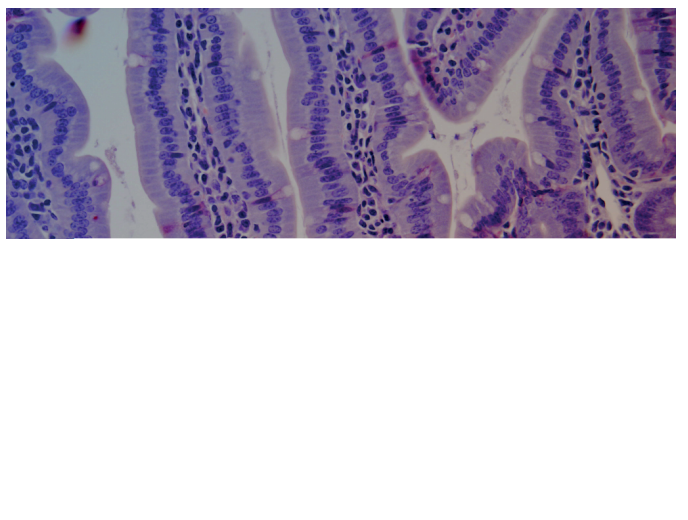

Figure 5.13: The $\boldsymbol{i F A B P} \boldsymbol{p}$-IL-9 transgene enhances basal jejunal mast cell activity in IL-3-deficient conditions. (A) serum mMCP-1 and (B) Jejunal CAE+ cells were determined in naive mice of the specified genotype. Pictures are representative sections of CAE staining from one high power field (HPF). Lines represent mean \pm SEM of ( $n=17$ mice/group) combined from 3 separate experiments using $(n=5-7$ mice/group). Data were analysed using One-way ANOVA with Tukey's post-test; ${ }^{*} \mathrm{p}<0.05,{ }^{* *} \mathrm{p}<0.01$ 
Next, we compared the development of allergic diarrhoea in $\mathrm{Tg}+/+, \mathrm{Tg}-/-$ and $\mathrm{nTg}-/-$ mice. After two i.p. primes with OVA, mice of all three genotypes had approximately $2 \mu \mathrm{g} / \mathrm{mL}$ Total IgE and OVA-specific IgE levels were similar (Figure 5.14D-F). With multiple i.g. OVA challenges, OVA-specific IgE levels increased to similar levels in the three OVA-fed groups (Figure 5.14D) and Total IgE levels increased to $4-6 \mu \mathrm{g} / \mathrm{mL}$ in all three groups of OVA-fed mice by the experiment end-point (Figure 5.14E-G). Similar to our previous observations in IL-3-/- mice, nTg-/- mice did not develop diarrhoea (Figure 5.14A). Tg-/- mice also did not exhibit symptoms of diarrhoea (Figure 5.14A). mMCP-1 levels were low in PBS-fed nTg-/- mice and did not increase during gavage challenge (Figure $5.14 \mathrm{~B}$ ). $\mathrm{Tg}+/+$ and $\mathrm{Tg}-/-$ mice again had elevated baseline mMCP-1 levels compared to $\mathrm{nTg}-/$ - mice and these remained low in PBS fed groups throughout the experiment (Figure 5.14B). OVA-fed mice of all genotypes had 100- to 200-fold increases in serum mMCP-1 levels compared to PBS-fed counterparts (Figure 5.14B) and these were highest in OVA-fed $\mathrm{Tg}+/+$ mice, reaching $\approx 50000$ $\mathrm{ng} / \mathrm{mL}$ following the $10^{\text {th }}$ i.g. OVA challenge (Figure 5.14B). While mMCP-1 levels in $\mathrm{Tg}$-/- mice were elevated over $\mathrm{nTg}$-/- mice by ten-fold after challenge 5, their mMCP-1 levels did not reach the levels in $\mathrm{Tg}+/+$ mice at any post-challenge point (Figure 5.14B). When we examined jejunal mast cell infiltration at the experimental end-point, jejunal mast cell density was highest in OVA-fed $\mathrm{Tg}+/+$ mice (Figure $5.14 \mathrm{C}$ ) and the number of mast cells correlated approximately with serum mMCP-1 concentration (c.f. figure 5.14B and C). When we examined mesenteric lymph node cell expression of IFN- $\gamma$, IL-3, IL-4, IL-5 and IL-13 upon restimulation with OVA no significant differences were observed IL-3 between $\mathrm{Tg}+/+$, $\mathrm{Tg}-/-$ and $\mathrm{nTg}-/-$ cultures except for the absence of IL-3 in IL-3 deficient mice (Appendix Figure 8.6A - E). These data suggest that high local IL-9 levels were not sufficient compensation to permit oral allergen-induced mastocytosis in the absence of IL-3. 

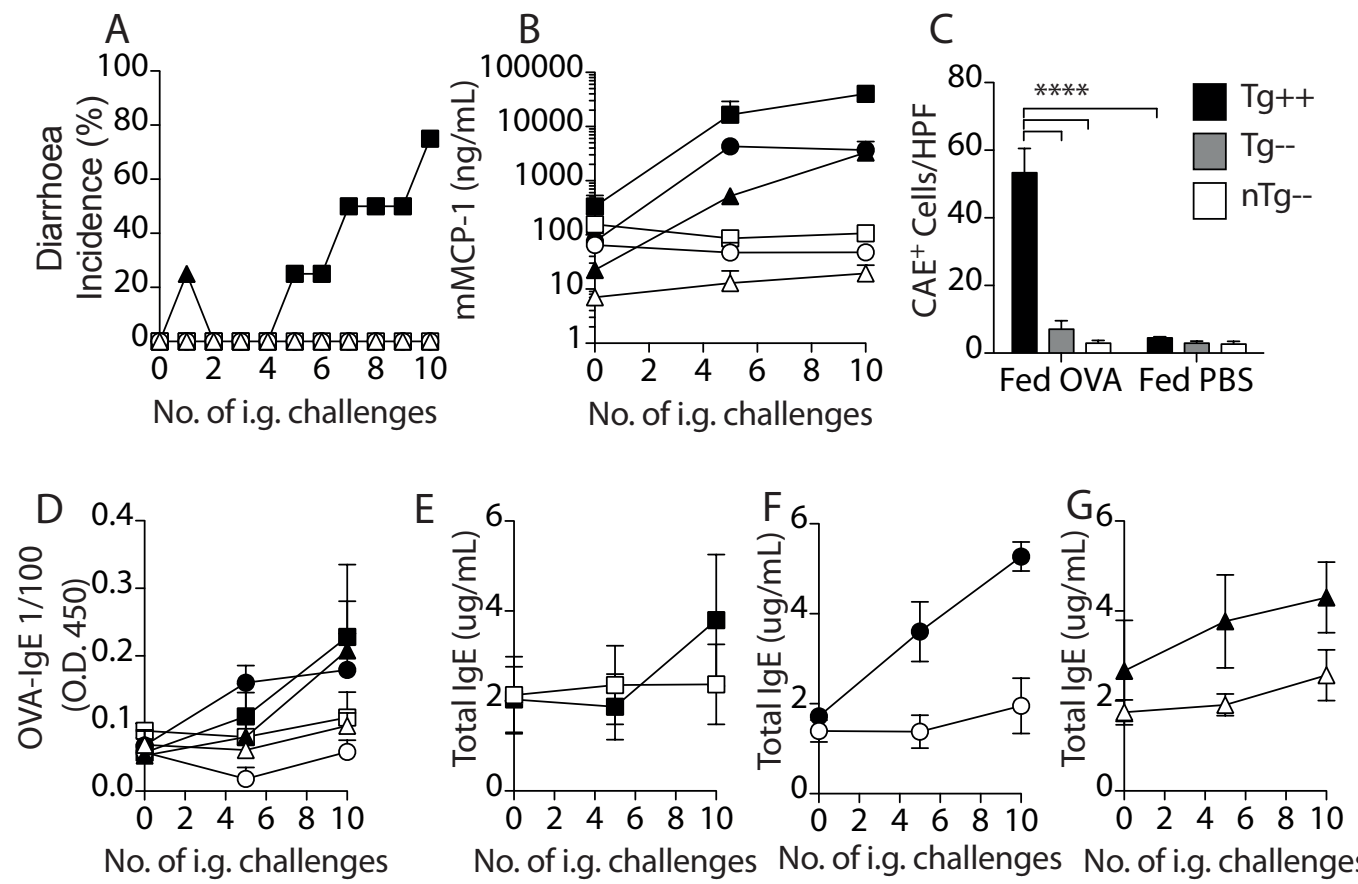

No. of i.g. challenges No. of i.g. challenges No. of i.g. challenges

Figure 5.14: IL-3 is required for diarrhoea development in mice with high basal mast cell activity. OVA-sensitised Tg+/+ (Squares), Tg-/- (circles), nTg/- (triangles) mice ( $n=4 /$ group) were fed with OVA (black) or PBS (white). (A) Diarrhoea development was monitored after oral gavage. (B) During the experiment, mice were bled after the stated number of challenges and serum mMCP-1 levels were determined by ELISA. (C) At the experiment end-point, jejunal sections were stained for CAE + cells in the indicated groups. (D) OVA-specific IgE and (E-G) Total IgE were examined in each strain. Data are shown from a single experiment and represent group mean \pm SEM. Data were compared using Two-way ANOVA with Bonferroni's post-test; $* * * * \mathrm{p}<0.0001$. 
With these experiments we demonstrated that IL-3-/- mice had impaired allergeninduced jejunal mast cell infiltration. Next we tested whether one IL-3 allele was sufficient to permit oral allergen-induce mastocytosis, or whether both IL-3 alleles were required. $i F A B P p$-IL-9 mice are predisposed to oral sensitisation[57], so in this experiment, mice were orally challenged seven days after their i.p. prime. In this setting, when $\mathrm{Tg}+/+$ mice were orally challenged with OVA, disease penetrance did not reach 100\%, precluding interpretation of the impact of IL-3-heterozygosity on diarrhoea (Figure 5.15A). However, we were able to investigate the relative level of oral potentiation that had occurred by examining serum mMCP-1, IgE and jejunal mast cell infiltration. We observed that despite similar OVA-specific IgE levels at the experiment end-point (Figure 5.15C), jejunal mastocytosis was only significantly elevated in $\mathrm{Tg}+/+$ mice, indicating that the loss of one IL-3 allele resulted in a partial impairment in intestinal mast cell infiltration (Figure 5.15B).

Numbers of $\mathrm{CAE}+$ cells were higher in $\mathrm{Tg}+/-$ mice compared to naive $\mathrm{Tg}+/+$ mice (shown by the dashed line in figure 5.15B), indicating that the increase in mast cells was not due to the presence of the transgene alone (Figure 5.15B). In this modified experimental system, Total IgE did not increase after i.g. OVA challenge (Figure 5.15D). i.g. OVA challenge caused mMCP-1 levels to increase in all groups of mice. Following the $9^{\text {th }}$ challenge mMCP-1 levels corrrelated with the number of CAE + cells, and not with OVA-IgE O.D. values (c.f. figure 5.15B and E, versus figure 5.15B and C). These data indicate that the loss of one IL-3 allele caused a partial reduction in allergen-induced intestinal mastocytosis and further suggest that the IL-9 transgene was able to increase oral-allergen induced mast cell activity in IL-3 heterozygous conditions. 

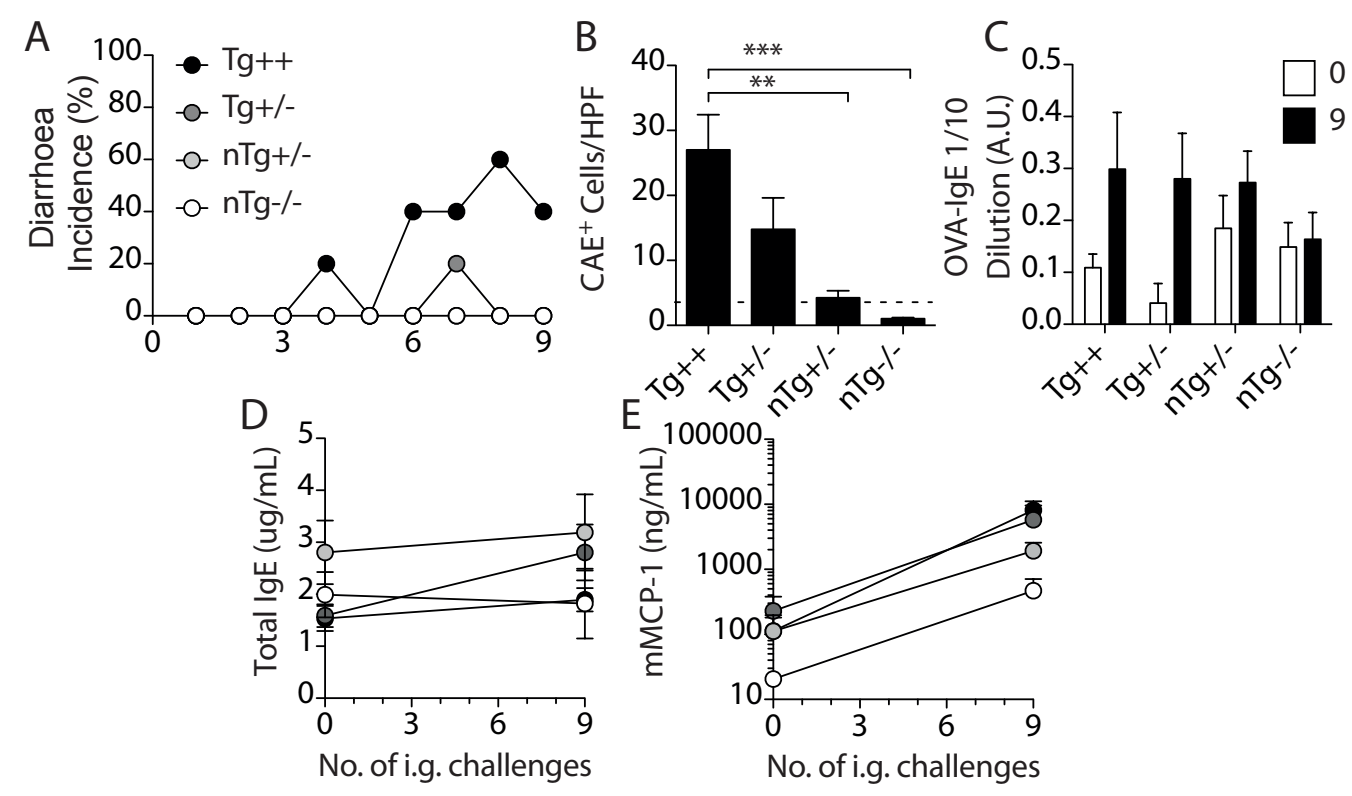

Figure 5.15: Lack of a single IL-3 allele causes a partial defect in allergeninduced intestinal mastocytosis. (A) Seven days after a single i.p. sensitisation, mice ( $\mathrm{n}=5$ /group) were fed $50 \mathrm{mg}$ OVA and monitored for diarrhoea development. (B) At the experimental end-point, jejunal CAE + cells were quantified in each strain. The dashed line shows the mean number of mast cells in naive $\mathrm{Tg}+/+$ mice sectioned in this experiment. (C) Before (0) and after (9) nine oral OVA challenges sera were analysed for OVA-specific IgE, (D) Total IgE and (E) mMCP-1 by ELISA. Data are represented by group mean $\pm \mathrm{SEM}$ ( $\mathrm{n}=5$ mice/group) from a single experiment. ${ }^{* *} \mathrm{p}<0.01,{ }^{* * *} \mathrm{p}<0.001$ by One-way ANOVA with Tukey's post-test.

\subsubsection{Assessing the role of IL-3 in i.v.-induced intestinal al- lergy}

In our studies with GK1.5 we observed that after a rest period, i.v. challenge of orally primed mice resulted in diarrhea. We sought to expand on these studies by quantifying the relative level of diarrhea by measuring the fur matting around the anus of mice following i.v. challenge. We also examined whether IL-3 was required for the i.v.-induced diarrhea. Mice were sensitized to OVA plus alum and then orally challenged 10 times with OVA, at which point $75 \%$ of OVA challenged IL-3+/+ mice had diarrhea (Figure 5.16A). Three days later, mice were i.v. challenged with 100 
$\mu \mathrm{g}$ OVA and all sensitized groups exhibited hypothermia (Figure 5.16B). However, in contrast to the results of Figure 5.7, mice in all groups, including naive animals exhibited some fur matting (Figure 5.16C and D). While the fur matting was most pronounced in the orally primed IL-3+/+ mice, mock-primed wildtype mice also exhibited significant fur matting and diarrhea (Figure $5.16 \mathrm{C}$ and D). The average amount of fur matting was lower in the sensitized IL-3 deficient mice, despite at least as severe i.v.-induced anaphylaxis (Figure 5.16B - D). Taken in conjunction with the results of Figure 5.7, non-allergic parameters seem to contribute to diarrhea and fur matting in this context, such as introduction of the rectal probe and the pre-heating step. Therefore i.v.-induced fur matting may lack the sensitivity required to measure the severity of disease, but could prove useful with further optimisation or without the rectal probing step. With these preclusions in mind, the results of Figure 5.16 and Figure 5.7 indicate that IL-3-dependent oral potentiation exaccerbates, rather than is necessarily required for i.v. allergen-induced diarrhea.

Taken collectively, our findings illustrate that IL-3, in addition to the roles established for IL-4 and IL-9[57, 295], is involved in oral allergen-induced jejunal mast cell infiltration. 


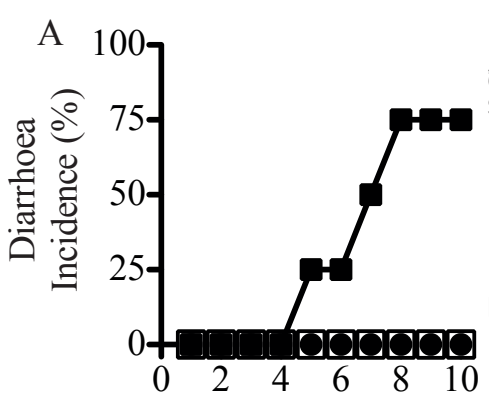

No. of ig challenges

$\mathrm{C}$
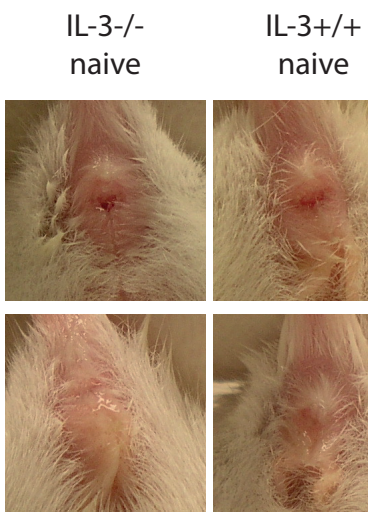

$\mathrm{D}$
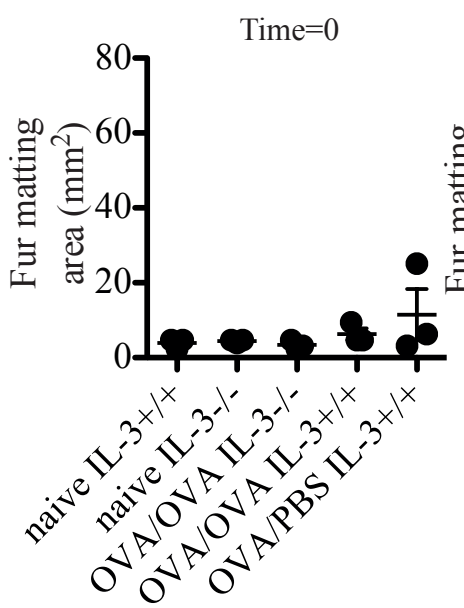
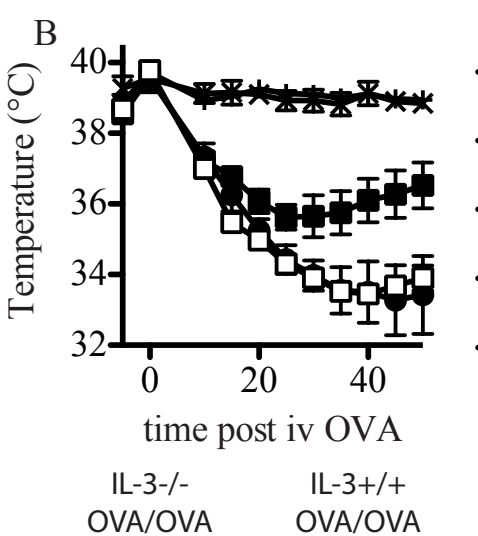

IL-3+/+

OVA/PBS
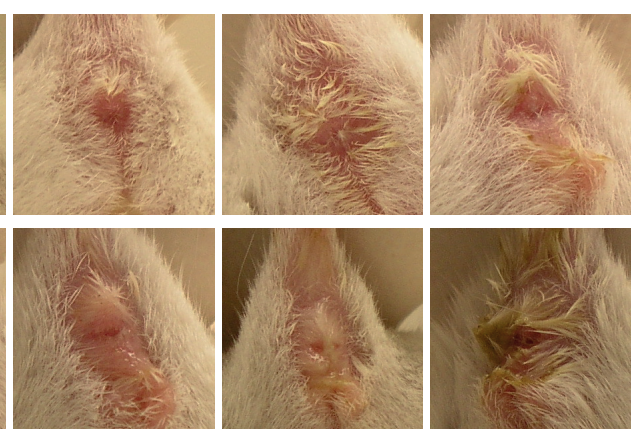

Time $=50$

Time $=20$
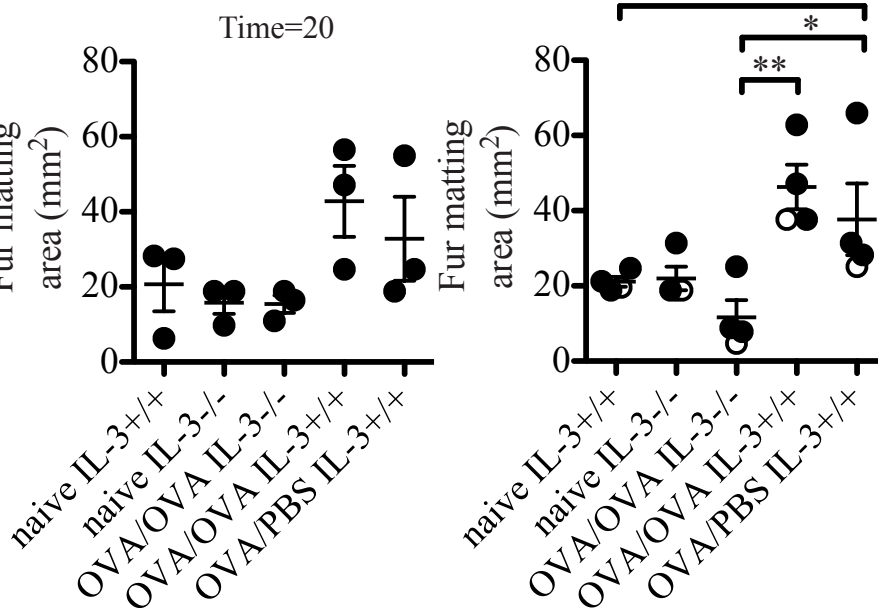

Figure 5.16: Systemic anaphylaxis and fur matting in IL-3 sufficient and deficient mice. (A) Mice were sensitized and orally challenged with OVA. (B) Three days after the final oral challenge mice were i.v. challenged with $100 \mu \mathrm{g}$ OVA. Naive mice also received i.v. $100 \mu \mathrm{g}$ OVA. (C) Photographs were taken 50 minutes after i.v. challenge. (D) Zero, twenty and fifty minutes after i.v. challenge the area of fur matting was calculated using $\left.\pi^{*}\left((\mathrm{x} / 2)^{*}(\mathrm{y}) / 2\right)\right)$; where $\mathrm{x}$ and $\mathrm{y}$ are the horizontal and vertical diameters of fur matting around the anus. Data are represented by group mean \pm SEM ( $\mathrm{n}=4$ mice/group) from a single experiment. ${ }^{* *} \mathrm{p}<0.01,{ }^{* * *} \mathrm{p}<0.001$ by One-way ANOVA with Tukey's post-test. White symbols in (D) represent mice that were not measured at earlier timepoints. 


\subsection{Discussion}

In the model we used, multiple oral feeds with OVA are required for mice to present with diarrhoea. Within the first three challenges, antigen-specific CD4+ T cells upregulate intestinal homing molecules, jejunal IL-4 mRNA levels become elevated, intestinal antigen-specific IgE titres rise, mMCP-1 mRNA transcripts are more frequent and mastocytosis is observed[38, 299]. We refer to this process as oral potentiaton and these changes are causatively or correlatively linked with the development of disease[37]. The aim of the experiments in this chapter was to clarify the immune processes involved in oral potentiation. Initially, we examined the contribution of CD4+ T cells to allergen-induced mastocytosis. We then examined the role of IL-3 in the allergen-induced intestinal mast cell response.

\subsubsection{The role of $\mathrm{CD} 4+\mathrm{T}$ cells in oral potentiation}

Our first studies were proposed based on experimental evidence implicating local $\mathrm{CD} 4+\mathrm{T}$ cells in oral potentiation. In three similar models, oral challenge of presensitized mice increases Th2-associated cytokine levels in the intestinal tissues, and causes specific enhancement of Th2-type cytokines upon restimulation of CD4+ T cells. This Th2 cytokine profile is observed in mice orally challenged with OVA after: i) intraperitoneal sensitization with OVA plus alum adjuvant[37, 38, 57, 299]; ii) subcutaneous sensitization with OVA plus complete Freund's adjuvant[58]; and iii) oral sensitization with OVA plus cholera toxin[298]. This profile is also observed when milk proteins or peanut allergens are used in similar models that also result in diarrhoea, intestinal permeability and vascular leak[149, 456]. Further, in all of these oral challenge models, mast cell and eosinophil influx follow oral challenge[37, $42,58,149,456]$. In all three OVA-challenge models, the allergic response is critically dependent on IL-4/STAT-6 signalling, and in the model we have employed, has been shown to be dependent specifically on $\operatorname{IgE}$ and mast cells[57, 58, 295, 298].

In our studies, we have confirmed a central role for CD4 $+\mathrm{T}$ cells in the development of diarrhoea and identified two different roles for CD4+ T cells in oral potentiation. When CD4+ T cells were depleted systemically prior to OVA feeding, oral potenti- 
ation did not occur (Figure 5.1). Allergen-induced mast cell activity was essentially ablated in CD4-depleted mice: mMCP-1 production was 100-fold lower and jejunal mast cell numbers averaged two per high power field, compared to approximately 35 in mock-depleted orally challenge mice. This is similar to the number we observed in unchallenged mice in other experiments. IgE production was also diminished relative to non-depleted mice, as would be expected. However, this alone was not sufficient to explain the impairment in allergen-induced mast cell recruitment, because hyper-primed, CD4-depleted mice that had $\mathrm{IgE}$ levels sufficient for disease exhibited delayed disease onset (Figure 5.4). These findings lead us to conclude that intestinal mastocytosis is a process independent of augmented IgE production. A previous report demonstrated that treatment of mice with FTY720, a drug that causes accumulation of lymphocytes in lymph nodes and Peyer's patches and prevents their dissemination[457] does not prevent oral potentiation[299]. Taken in this light, we consider the mast cell component of disease to be controlled by intestinally-localised Th2 mast cell-inducer (Th2m) cells, which are functionally distinct from IgE-promoting follicular T helper ( $\mathrm{TFH})$ cells.

\subsubsection{Distinct roles for CD4+ $\mathrm{T}$ cells in intestinal mastocy- tosis and IgE generation}

The requirement for $\mathrm{CD} 4+\mathrm{T}$ cell function in establishing the local inflammatory milieu in the intestine in addition to supporting $\operatorname{IgE}$ production is a potentially important feature of the allergic process. Current evidence suggests that IgE production is controlled by IL-4-producing TFH cells found in lymph nodes[275] and lymph node- and tissue-localised CD4+ T cells are differently programmed and functionally distinct $[275,317,368,369]$. Following helminth infection, CD4+ T cells found in the lung produce increased levels of IL-13, and significantly less IL-21 than do CD4+ T cells in the lymph nodes[275, 317]. Further, the acquisition of TFH function, or programming to egress from the lymph node to alternate sites are in part dictated by $\mathrm{T}$ cell receptor (TCR) antigen affinity[369]. This bifurcation of antigen-experienced cells can be observed prior to the development of germinal centres soon after $\mathrm{T}$ cell activation[368]. Therefore, TFH cells and Th cells that enter the tissues may arise 
from separate precursor populations. In terms of the oral potentiation response, it is possible that populations of OVA-reactive CD4+ T cells that promote IgE production are distinct from those that control local mastocytosis. However, in the model we used for experimentation, mice received their first challenge four weeks after their initial sensitization. In this period, ample time existed for $\mathrm{T}$ cell redistribution to multiple sites. TFH cells can enter tissues, because adoptively transferred IL-21-reporterexpressing cells are found in the lungs of influenza-infected mice[367]. Furthermore, in a similar model to the one we used, both CD4+ Th2 cells and IgE producing B cells have been identified in the colonic lamina propria[58]. While IgE is not sufficient for mastocytosis on its own, interaction with B cells in the intestine may be required for Th2m cell function. However, irrespective of local B cell involvement, the data clearly indicate that CD4+ T cells control local intestinal mast cell infiltration.

\subsubsection{The role of $\mathrm{CD} 4+\mathrm{T}$ cells in disease maintenance}

While CD4+ T cell depletion prevented oral potentiation and disease development, CD4+ T cell depletion was not sufficient to tolerise mice to allergen. In our temporary depletion studies, mice were protected from disease only while their CD4+ T cells were absent; acute and transient oral allergen-induced diarrhoea was observed in mice soon (three challenges) after CD4+ $\mathrm{T}$ cells emerged in the periphery (Figure 5.3). It is known that $\mathrm{T}$ cells are involved in oral tolerance[137, 143, 458] so to some extent, this result was to be expected. Sensitized CD4-depleted mice also remained susceptible to i.v.-induced systemic anaphylaxis (Figure 5.2), so the initial sensitization was sufficient for systemic, but not intestinally-localised allergic responses. A similar potentiating role is attributed to CD4+ T cells in the development of allergen-induced airways hyperresponsiveness. In sensitized and subsequently CD4-depleted mice, challenge-induced airways eosinophilia is ablated and subsequent airways hyperresponsiveness is prevented[459]. Intriguingly, while eosinophilia is observed in lungs of intranasally allergen-challenged mice[294] and in the intestines of orally allergen-challenged mice[37, 42, 58], neither eosinophils nor IL-5 contribute to the CD4-dependent respective airways hyperresponsiveness or diarrhoea[37, 294, 459]. The parallels in the processes indicate that the events that permit the triggering of 
an allergic response in the lung or the gut mucosa are separate events from systemic sensitization.

The processes that maintain disease have also been investigated. IL-4 and IL-13, are critical in the oral potentiation period, but once disease is established systemic administration of anti-IL4 or anti-IL-13 antibodies does not prevent disease triggering[295]. Our findings are compatible with these findings. We observed that in the absence of CD4+ T cells, which are the most likely source of IL-4 and IL-13 required for oral potentiation, most mice remained susceptible to disease (Figure 5.5). Although the oral potentiation parameters remained elevated, we also observed an eventual diminution in disease incidence, and reduced mast cell activity in CD4-depleted mice, indicating that the long-term response was indeed still potentiated by CD4+ T cells (Figure 5.5). Taken together, the FTY720 studies in which oral potentiation was not inhibited[299], the partial attenuation in disease we observed following CD4-depletion (Figure 5.5), and segregation of IgE production from mastocytosis in our potentiation experiments (Figure 5.4), the data indicate that Th2m cells are required in the intestinal tissue itself. Although these findings indicate that intestinally localised Th2m cells are required for optimal oral potentiation, we also demonstrated that once established, the susceptibility conferred by oral potentiation is reasonably long-lived.

In our experiments, oral potentiation did not increase temperature loss induced by i.v. allergen administration (Figure 5.7 and 5.16). This contrasts with a previous report that indicated the severy of anaphylaxis increases in orally primed mice[37]. This may relate to the contributions of IgG-mediated processes to the anaphylactic response. While IgE-dependent systemic anaphylaxis is mast cell-dependent[43], IgG-bound basophils and macrophages can contribute to systemic anaphylaxis[40, 41, 47]. Additionally, systemic administration of antigen-specific-IgG1 can inhibit IgE-mediated anaphylactic responses induced orally in mice[67]. It is possible that significant differences in hypothermia would have been revealed if IgG-dependent processes were first neutralised in our studies. We also note that diarrhoea penetrance in the studies by Brandt et al. generally reached 100 percent within 6 challenges[37], whereas in our lab it can require ten or more challenges for it to be observed in all mice. Previous studies also demonstrate that low doses of allergen require more challenges to invoke diarrhoea[37]. It is possible that oral potentiation could occur faster if 
we used higher challenge doses and may similarly be required for oral potentiation to heighten the systemic response. We did, however, observe liquid stools and fur matting 10 to 25 minutes after i.v. allergen challenge of orally primed mice (Figure 5.7 ), which indicates that the anaphylactic response was more severe in orally-primed mice, in line with the findings of Brandt et al.[37]. However, as noted in the results section, although fur matting measurements indicated that it remained 'less severe' than orally-primed mice, we also observed diarrhea in some i.v. challenged naive mice (Figure 5.16), indicating that additional non-allergy associated parameters of the experimental regimen could contribute to the i.v.-induced diarrhea. Determining whether i.v.-induced intestinal anaphylaxis is severe enough to be observed without introduction of the rectal probe, and further, whether this requires sensitization, or is a property for which oral OVA-feeding is sufficient needs to be addressed in future studies. The heating requirement could be removed if allergen were administered i.p. instead of i.v. in mice and permit better characterization of the i.v.-induced intestinal allergic response.

\subsubsection{Clarifying the earliest events in the intiation of oral potentiation}

Determining whether the first step involved in oral potentiation requires the presence of CD4+ T cells or will occur in their absence will also be important to investigate in future studies. Allergen-reactive CD4+ T cells are recruited to the allergen-exposed intestine[38, 58], which argues that the local inflammatory milieu promotes CD4+ $\mathrm{T}$ cell recruitment. If the earliest events in the oral potentiation process require the presence of CD4+ T cells, the implications for disease therapy will be different than if the earliest events that promote CD4+ $\mathrm{T}$ cell responses are initiated as a preceding independent process. CD4 $\mathrm{T}$ cell activation in the mesenteric lymph node is thought to be a key step in the oral potentiation process[38], which predicates allergen and/or antigen-presenting cell transit to the mesenteric lymph node after oral challenge. OX40L-expressing dendritic cells are demonstrated as Th2-promoting cells in the intestine[434] and allergen binding to epithelial cells is associated with more severe intestinal allergic responses[139]. The way innate and non-haematopoeitic cells 
respond to allergen can influence the allergic process, so determining whether these changes occur in the absence of antigen-specific CD4+ T cells has the potential to determine the most appropriate targets for therapeutic intervention in atopic individuals.

There are a number of ways of further assessing the requirement for CD4+ $\mathrm{T}$ cells in the initiation of oral potentiation. First, if the CD4 $+\mathrm{T}$ cell population was depleted for a short period (two to three challenges), and then allowed to replenish, examining mastocytosis in the absence of further challenges would indicate whether or not the potentiation process is initiated by oral exposure in the absence of CD4+ T cells - Without further challenges, do mast cells infiltrate the intestine as CD4+ $\mathrm{T}$ cells re-emerge? If they do, this will provide evidence that oral potentiation is initiated in the absence of CD4+ T cells. As antibody treatment can be leaky, an adoptive transfer method could also be employed. Studies have demonstrated that adoptively transferring OVA-specific CD4+ $\mathrm{T}$ cells to naive mice, then feeding the mice OVA induces OVA-specific CD4+ T cell proliferation in mesenteric lymph nodes and causes their dissemination to the spleen[38]. In future work, mice could be depleted of CD4+ T cells, gavaged with OVA or PBS, and then receive OVA-specific CD4+ T cells once they were CD4-replete. If the transferred OVA-specific CD4+ $\mathrm{T}$ cells were preferentially sequestered to the jejunum, Peyer's patches or mesenteric lymph node of the OVA-fed group, this would indicate that their recruitment was initiated in their absence. Treating naive mice in a similar manner would be plausible, but may be confounded because oral exposure without sensitization inhibits Th2 polarisation[134, 143, 458]. This may affect the response of the adoptivelytransferred cells. Oral potentiation has been linked to CD4+ T cell $\alpha 4 \beta 7$ and CCR- 6 expression[38, 299], so monitoring these molecules on emerging CD4+ T cells in CD4replete mice could also link intestinal homing to disease. Lastly, it will be desirable to determine the frequency and activation status of OVA-specific CD4 $+\mathrm{T}$ cells in the gastrointestinal immune system of CD4-replete mice. These studies will have the potential to determine whether the earliest event in oral potentiation is CD4+ T cell dependent, or alternatively, if CD4 $+\mathrm{T}$ cells are simply a stepping-stone in an already catalysed process. 


\subsubsection{Requirement for oral potentiation for diarrhoea in dif- ferent contexts}

It is important to note that oral potentiation is not required for disease development in all allergy models. In rats, peripheral sensitization is sufficient to induce an antigenspecific IgE mediated response in the intestine[44, 54, 59]. The first intestinal exposure disrupts motility and causes diarrhoea if allergen is intubated directly into the duodenum or colon[44, 53, 59, 460]. Antigen-specific allergic intestinal responses have also been observed on first antigen challenge in subcutaneously sensitized mice[140], and those born to orally-sensitized dammes[20]. In systems very close to the model we have used, diarrhoea has been observed on the first challenge. In mice with elevated intestinal mast cell numbers that are predisposed to diarrhoea development, allergic diarrhoea has been observed without an oral potentiation period, but this is again rare[67]. There is at least one other report in which diarrhoea was observed on the first challenge of a mouse[299]. This suggests that in some circumstances, the presence of IgE and mast cells are sufficient for diarrhoea to develop. However, a direct correlation between intestinal mast cell numbers and the severity of the orally-induced anaphylactic response has been demonstrated[36], and mast cell numbers increase significantly following multiple gavages[37, 38]. Further, mice transgenically modified to have high basal mast cell activity[57] and mice with naturally increased mast cell numbers[39] demonstrate heightened allergic disease susceptibility. Collectively, these data suggest that intestinal disease is influenced by the level of mastocytosis, which is increased during the CD4+ T cell dependent establishment of the diarrhea-permissive local inflammatory milieu in the intestine.

Our studies have investigated the role of CD4+ T cells in the intestinal allergic process. We find that intestinal mast cell recruitment is controlled by local CD4+ T cell activity. CD4 $+\mathrm{T}$ cells also promote systemic IgE production. While these processes culminate in intestinally-localised allergic responses, they can be attributed to independent functions of CD4+ T cells. 


\subsubsection{The role of IL-3 in mast cell-dependent Th2 immu- nity}

Once we had determined a causal role for CD4+ T cells in the oral potentiation process we next investigated the potential involvement of IL-3 in intestinal allergy.

The rationale for investigating IL-3 comes from previous work identifying a role for IL3 in Th2-associated intestinal immunity and also in some models of hypersensitivity. Parenteral allergic responses, including mast cell-dependent[43] IgE-mediated passive cutaneous anaphylaxis and OVA-induced active systemic anaphylaxis proceed unencumbered in the absence of IL-3[455]. In contrast, symptoms of oxazolone-induced contact hypersensitivity and IgE-triggered serotonin release are reduced in IL-3-/mice[396, 455]. In wild-type mice, systemic administration of IL-3 increases jejunal mast cell deposition and systemic basophil generation[301, 311, 461], whereas blockade of IL-3 signaling prevents intestinal mastocytosis in response to parasite infection[462]. Both parasite infection and oral-allergen exposure induce intestinal permeability, mast cell recruitment and mMCP-1 production in mice[37, 50, 463]. Although IL-3 has been implicated in the regulation of IgE-mediated allergic reactions, the contribution of this cytokine to oral allergen-induced intestinal allergic responses has not previously been explored.

IL-3 is known to be produced by T cells[322, 464] and T cell derived IL-3 is known to promote mast cell development[465]. When we examined the potential involvement of IL-3 in a Th2-type response, we observed that culture with IL-4 increased the amount of IL-3 produced by anti-CD3-stimulated splenocytes. At least in part, this was due to IL-3 production by CD4+ T cells. Confirming a wealth of previous reports (e.g., $[465,466])$, we observed mast cell and basophil development from bone marrow cells cultured with IL-3. In contrast, while TSLP has recently been identified to promote IL-3-independent, IL-4-producing basophil development[429], only IL-3 gave rise to mast cells under the same culture conditions. Further, unlike the basophils that were present in cultures containing IL-3 and/or TSLP, the mast cells that developed did not express GFP, which reflects IL-4 production in the G4 mouse[273]. From these data, we conclude that IL-4 promoted IL-3 production by T cells. We also conclude that CD4+ T cells may contribute to intestinal mastocytosis in Th2 settings through 
the production of IL-3.

\subsubsection{The role of IL-3 in intestinal food allergy}

IL-3 deficiency impaired the development of an intestinal allergic response in mice. When we monitored disease development in sensitized and orally challenged IL-3-/mice, they neither developed diarrhoea nor lost plasma volume; faecal pellets and caecal content were also retained. While mastocytosis was diminished in IL-3-/- mice relative to wild-type mice, oral challenge increased systemic $\operatorname{IgE}$ titres, indicating that CD4-dependent responses still occurred. We also showed that IL-3-deficient mice remained susceptible to systemic anaphylaxis, as established previously[455]. From these data, we conclude that IL-3 was specifically required for intestinal allergic mast cell responses.

When we assessed the relative contribution of IL-3 and IL-9 to intestinal mastocytosis, our data confirmed that IL-3 was required for intestinal mast cell deposition. Overexpression of IL-9, which augments mastocytosis in IL-3-sufficient mice[57, 312], also increased jejunal mast cell numbers in IL-3-deficient mice, but was not sufficient to permit disease, or bypass the requirement of IL-3 in allergen-induced mastocytosis. Not only did this confirm that IL-3 was required for allergen-induced intestinal mast cell recruitment, but it also provided further evidence that antibody production and intestinal mast cell recruitment were independent processes. Similar observations have been made in IL-9-deficient settings[57] and support our conclusions. To confirm that the difference in IgE levels in IL-3-deficient and IL-3-sufficient mice do not underlie their protection from disease, testing whether IL-3-deficient mice develop disease when given IL-3 exogenously, as done previously in parasite infection settings[301, 311, 461], will validate the findings of our study. However, as IgE levels were similar in $\mathrm{Tg}+/+$ and $\mathrm{Tg}-/-$ mice, but only $\mathrm{Tg}+/+$ mice presented with diarrhoea, we consider it unlikely that discrepancies in IgE titres underlie the differences in the intestinal mast cell response of IL-3+/+ and IL-3-/- mice. From these data, we conclude that oral allergen-induced mastocytosis is partially dependent on IL-3. 


\subsubsection{The source of IL-3 that drives allergic intestinal mas- tocytosis}

Intestinal CD4+ T cells are a probable source of the IL-3 that promoted the allergic intestinal mastocytosis. Intestinal helminth infection normally induces 1000fold increases in IL-3, but if T cells are first depleted, Peyer's patch IL-3 levels are 10-fold lower than in naive mice[315]. Both IL-3 and T cells are required for helminth-induced intestinal mastocytosis, and this expedites the expulsion of some parasites[307, 309, 311, 314, 462, 467]. Thus, intestinal $\mathrm{T}$ cells are a potent source of mast cell-inducing IL-3. This will, however, need to be validated in a food allergy model, because committed mast cell progenitors are found in the blood and bone marrow $[314,468,469]$. It is therefore possible that IL-3 is required systemically, rather than locally, to promote intestinal allergy. Examining mast cell progenitor deposition in the intestines of IL-3-/- mice, as done previously in disease-resistant IL-9-/mice[57], may indicate whether IL-3 is required locally or plays its most important role before mast cell progenitors reach the intestinal tissue.

The contribution of CD4+ $\mathrm{T}$ cell-derived IL-3 to intestinal mastocytosis will be a focus for future studies. Adoptive transfer of ex vivo-restimulated CD4+ T cells from diarrhoetic wild-type mice renders otherwise naive mice susceptible to diarrhoea[38]. Transferring IL-3-sufficient or deficient OVA-specific CD4+ T cells to IL-3-deficient mice would determine whether CD4+ T cells were a sufficient source of IL-3 to promote mastocytosis and permit disease. DO11.10 mice have an enriched pool of CD4+ T cells specifically recognising the OVA323-339 epitope[470] and develop allergic diarrhoea in the model we used[471]. Adoptive transfer of IL-3-sufficient or IL-3-deficient DO11.10 CD4+ T cells to IL-3-/- mice prior to sensitization, then monitoring disease and mMCP-1 levels in the challenge model will elucidate whether or not CD4+ T cell IL-3-production is sufficient to promote allergic intestinal mastocytosis. 


\subsubsection{Additional potential effects of IL-3 on intestinal mast cell function}

In addition to the reduction in jejunal mast cell numbers in IL-3-/- mice that we observed, it is also possible that IL-3 alters the phenotype of intestinally-localised mast cells. While mouse mast cells differentiate in response to IL-3, exposure to additional cytokines, like IL-4 and IL-9, are required for mast cell mMCP-1 expression[472]. In humans, the process is different. IL-3 promotes the activation, rather than the differentiation of pure mast cell populations, and IL-6 and IL-10 synergise with SCF to promote human mast cell development[314]. IL-6 and IL-10 promote the development of less differentiated mast cells in mice[314], such that IL-3 may promote the maturation as well as the expansion of intestinal mast cells. Determining the mediator content of intestinal mast cells and the role of IL-3 in human intestinal allergies will be important in future studies.

\subsection{Summary}

We have confirmed a key role for CD4+ T cells in local oral potentiation. We identify that CD4+ T cells contribute to estalishing the local inflammatory milieu in the jejunum in a manner that is additional to their role in IgE production. We determined that the allergic response is optimal in the presence of CD4+ T cells, but oral potentiation is sufficient for continued susceptibility to oral allergen challenge responses. IL-3 was required for diarrhoea to manifest and was required in addition to IL-9 for optimal intestinal mastocytosis. 


\section{Chapter 6}

\section{Examining milk allergenicity in Th2 immune response models}

\subsection{Introduction}

\subsubsection{Rationale for examining goat milk allergenicity}

Milk allergy in the paediatric population is reasonably common. Of the five percent of children in the US that have had an allergic episode, approximately half will have been triggered by cows milk[9]. A similar incidence is reported in New Zealand infants[473]. While there are significant similarities in their composition, goat milk is less allergenic than cow milk in numerous experimental settings. In this, goat milk offers the potential to be used in a hypoallergenic milk formula that will result in fewer allergies compared with cow milk-based formulae.

Goat milk has been shown to be a weaker allergen than cow milk in a number of models. In mice sensitized orally to milks, splenocyte milk-specific IL-4 production is lower, diarrhoea less frequent and the severity of disease induced by oral feeding is lower in goat milk-fed mice[234]. In some studies, guinea pigs demonstrate more severe anaphylactic responses to cow milk than goat milk[141]. Heat-treating goat milk diminishes its allergenicity in guinea pigs to a greater extent than cow milk[238]. However, goat milk can sensitize, and induce significant allergic responses in both experimental and clinical settings[221, 238]. Therefore, to develop a hypoallergenic infant formula, clarification of the specific components of goat milk that contribute to its allergenicity is required.

In the literature, several milk components have been identified as potential sources of allergenicity. First, the casein content, and specifically the $\alpha$ s1-casein content, 
influences the allergenicity of milk. This is observed in multiple settings. Milk sourced from goat breeds in which $\alpha$ s1-casein levels are low results in reduced allergic disease incidence and lower severity in guinea pig studies[249]. In another study, goat milk with 10-fold lower $\alpha$ s1-casein content than cow milk also resulted in lower incidences and less severe allergic responses in mice[234]. One study in mice has shown that allergenicity of cow milk can be reduced by modifying its casein:whey balance from 80:20 to 60:40[250]. Intriguingly, milks with reduced or naturally low $\alpha$ s1-casein content generate less BLG-specific antibody and reduce the allergic response triggered by BLG in the intestine $[249,250]$. This suggests that while the casein content of milks is important, both BLG and caseins are implicated in the differential allergenicity of cow and goat milks. However, these studies may also indicate that the relative concentrations of specific proteins, rather than the intrinsic allergenicity of homologues on a protein-by-protein basis, influence milk allergenicity. Therefore, we sought to clarify whether differential allergenicity could be attributed to specific components of goat and cow milk. Finally, we considered that rather than weaker allergenicity, goat milk may have some additional allergy-suppressing properties that inhibit Th2 responses, including those to milk components. Therefore, in this section, we aimed to clarify which components and properties of goat milk influence its comparatively weak allergenicity. 


\subsection{Aims}

In this chapter we used the models of Th2 immunity characterised in the previous chapters to evaluate the allergenicity of milk components. Specifically, our objectives were to determine:

1) If there are differences in the intrinsic allergenicity of homologous cow and goat milk proteins;

2) If goat milk can suppress Th2 responses;

3) If goat BLG can induce intestinal mast cell responses in sensitized mice;

4) If cow and goat BLG differentially affect orally-induced allergic intestinal mast cell activity. 


\subsection{Results}

We began our studies using the pTh2 assay to compare the Th2 response induced by isolated milk proteins. As $\alpha$ s1-casein and BLG are major milk allergens absent or low in human milk, we compared the intrinsic allergenicity of these cow and goat homologues.

\subsubsection{Examining pTh2 responses to $\beta$-lactoglobulin}

In work carried out prior to this study, our lab observed that Th2 responses to food allergens were generally weak (Figure 1.3). Therefore, we examined whether Th2 responses could be increased if more allergen was administered. In this and all subsequent pTh2 assays, mice were anaesthetised, and then received the stated dose of allergen intradermally in the ear pinna. Seven days later, the draining lymph node was excised and the Th2 response was analysed.

First, we examined the Th2 response to either $1 \mathrm{mg}$ or $100 \mu \mathrm{g}$ of cow BLG. When we examined the total number of cells infiltrating the lymph node, approximately twice as many cells were detected when $1 \mathrm{mg}$ was used compared with $100 \mu \mathrm{g}$ (Figure 6.1A). The number of $\mathrm{B}$ cells and $\mathrm{CD} 4+\mathrm{T}$ cells increased in mice that received 1 mg cow BLG (Figure 6.1B and C). We also observed that $1 \mathrm{mg}$ BLG increased the proportion of $\mathrm{GFP}+\mathrm{CD} 4+$ cells, resulting in an approximately 15-fold increase in Th2 cell numbers which was not observed at $100 \mu \mathrm{g}$ (Figure 6.1D and E). These data suggest that $1 \mathrm{mg}$ cow BLG was immunogenic and allergenic in the pTh2 assay, while $0.1 \mathrm{mg}$ was sub-immunogenic.

Next we compared the response for cow and goat BLG. In this assay, we included a $2.5 \mathrm{mg}$ dose. When 1 or $2.5 \mathrm{mg}$ BLG was injected, irrespective of the species source, we observed increased lymph node cellularity, which could largely be attributed to B cell and CD4+ T cell hyperplasia (Figure 6.2A-C). We also observed dose-dependent increases in the proportion and number of Th2 cells, and these increases were similar for both species ( $p>0.05$ for all parameters; Figure 6.2 A-C, E and F). These data suggest that cow and goat BLG have equivalent allergenicity in the pTh2 assay. 


\subsubsection{Examining pTh2 responses to casein proteins.}

Caseins are also major milk allergens, with between 85 and 100 percent of milk allergic individuals exhibiting $\operatorname{IgE}$ binding to casein proteins[34, 183]. As significant IgE binding to one casein protein, $\alpha$ s1-casein, is frequently observed[182], we next examined the allergenicity of $\alpha$ s1-casein, and that of the whole casein fraction.

In our initial assays, we had difficulties with the solubility of the caseins, so we tested the amount of protein we were administering using standard protein quantitation assays. Solubilised HPLC-purified goat $\alpha$ s1-casein generated a lower O.D. reading compared with the equivalent mass of cow $\alpha$ s1-casein, but gave similar $\mathrm{BCA} \mathrm{Cu}^{2}+$ reduction results (Figure $6.3 \mathrm{~A}$ and $\mathrm{B}$ ). The same mass of goat $\alpha \mathrm{s} 1$-casein and whole goat caseins gave both similar absorbances and $\mathrm{BCA} \mathrm{Cu}^{2+}$ reduction values (Figure $6.3 \mathrm{~A}$ and $\mathrm{B})$. These aliquots were used directly in one of the three assays used to compile Figure 6.4. An additional aliquot of goat $\alpha s 1$ casein and the whole casein fraction were frozen, and subsequently used for the assay shown in figure 6.5. Immunisations were performed based on the weighed mass, rather than results of either of the protein assays.

When mice were immunized with HPLC-purified $\alpha$ s1-casein, there was a minor increase in total cellularity in response to $1 \mathrm{mg}$ cow and goat $\alpha$ s1-casein (Figure 6.4A), but only cow reached statistical significance. We did not observe increases in CD4+ T cell numbers in mice immunised with either cow or goat $\alpha$ s1-casein (Figure 6.4 B). In the mice that received $1 \mathrm{mg}$ of either protein, we observed statistically non-significant increases in B cell number, and small, but significant increases in the proportion and number of Th2 cells (Figure 6.4B, D and E). At no dose did we observe differences between the two $\alpha$ s1-caseins ( $<0.05$ for all parameters). These data suggest that $\alpha$ s1casein isolated from cow, or from goat milk have similar and low allergenicity, in terms of their ability to initiate Th2 cell development in the absence of adjuvant.

Next we tested whether caseins are more allergenic as a whole, than the isolated $\alpha$ s1casein. When we compared $333 \mu \mathrm{g}$ masses of goat $\alpha \mathrm{s} 1$ and whole caseins, we observed similar immunogenicity, as there were minor, or no difference in the total cell infiltrate, $\mathrm{B}$ cell infiltrate or CD4+ T cell infiltrate in draining lymph nodes (Figure 6.5A-C). 
However, we observed a clear difference in the Th2 response: Equivalent masses of $\alpha$ s1-casein generated $\approx 2000 \mathrm{Th} 2$ cells, whereas $\approx 6000$ were induced by the equivalent mass of the whole casein fraction (Figure 6.5E). Similar relationships were observed for proportional Th2 responses (Figure 6.5D). These data suggest that wholegoat caseins have higher intrinsic allergenicity than goat $\alpha$ s1-casein alone.

We next compared the allergenicity of cow and goat whole caseins. When we immunized mice with $1 \mathrm{mg}$ of whole casein fractions we observed pronounced increases in lymph node hyperplasia, B cell, CD4+ T cell and Th2 cell expansion (Figure 6.6A-E). Both cow and goat whole caseins increased the proportion and number of Th2 cells present in lymph nodes compared to non-draining lymph nodes, most significantly at the highest dose $(1 \mathrm{mg}$; Figure $6.6 \mathrm{D}$ and $\mathrm{E})$. While there may have been a small increase in Th2 proportion for cow over goat at $1 \mathrm{mg}$, no statistically significant difference was detected in the proportion or number of Th2 cells at any dose ( $p>0.05$ at all doses). Therefore, these data suggest that cow and goat whole caseins both induced Th2 cell development and did so to similar extents in the pTh2 assay. 


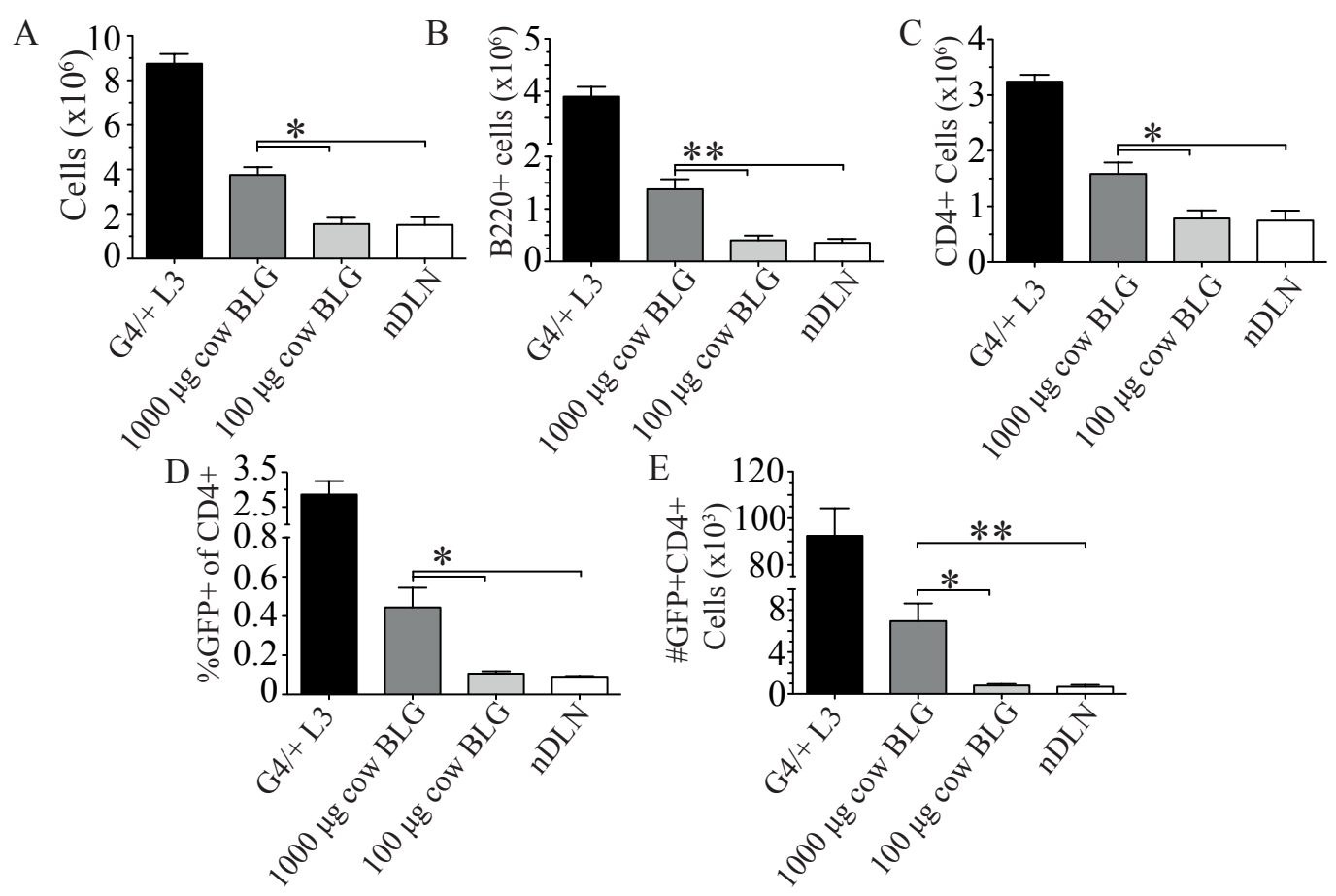

Figure 6.1: Dose-dependent Th2 response to cow $\beta$-lactoglobulin. Mice ( $\mathrm{n}=3$ /group) were injected intradermally with 600 dead $i$ L3 $N$. brasiliensis larvae (G4/+ L3), $1000 \mu \mathrm{g}$ cow BLG, or $100 \mu \mathrm{g}$ cow BLG. Seven days later, draining lymph nodes were taken, and one non-draining node from each group was taken (nDLN) and analysed by trypan blue exclusion test for (A) total cell number and after flow cytometric characterization, the number of (B) B220+ cells, (C) CD4+ T cells and the (D) proportion and (E) number of CD4+ T cells that were GFP + . Bars show mean \pm SEM of groups of $(n=3)$ mice from one of two similar experiments. Data were compared using One-way ANOVA with Tukey's post-test. ${ }^{*} \mathrm{p}<0.05,{ }^{* *} \mathrm{p}<0.01$. 

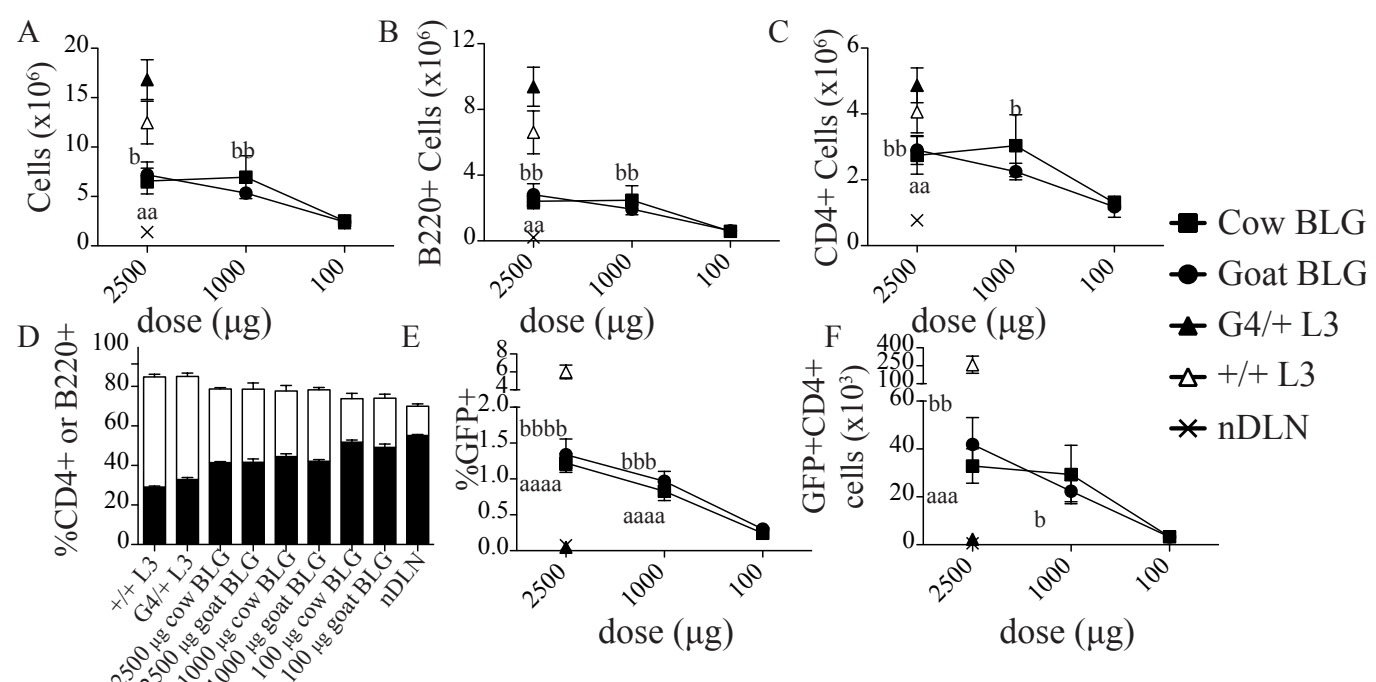

Figure 6.2: Similar Th2 response to cow and goat $\beta$-lactoglobulin. G4/ + mice $(\mathrm{n}=5$ /group) were immunized intradermally with the stated dose of cow (squares) or goat (circles) BLG. Control $+/+$ (white triangles) and G4/+ (black triangles) mice were immunized with 600 dead $i$ L3 $N$. brasiliensis larvae. Non-draining (nDLN) lymph nodes (crosses) were used to reflect the assay baseline. (A) Total cell numbers were calculated by trypan blue exclusion, and the number of (B) B220 and (C) CD4+ T cells were back calculated based on the (D) proportion of live cells that were B220+ (White bars) or CD4+ (Black bars). (E) The proportion and (F) number of CD4+ T cells that expressed GFP, as a surrogate of IL-4 protein induction, were quantified. Data are shown from a single experiment and the dose response was analysed using Two-way ANOVA with Bonferroni's post-test; b p $<0.05$, bb p $<0.01$, bbbb $\mathrm{p}<0.0001$ cow BLG vs. nDLN; aa $\mathrm{p}<0.01$, aaaa $\mathrm{p}<0.0001$ goat BLG vs. nDLN. 

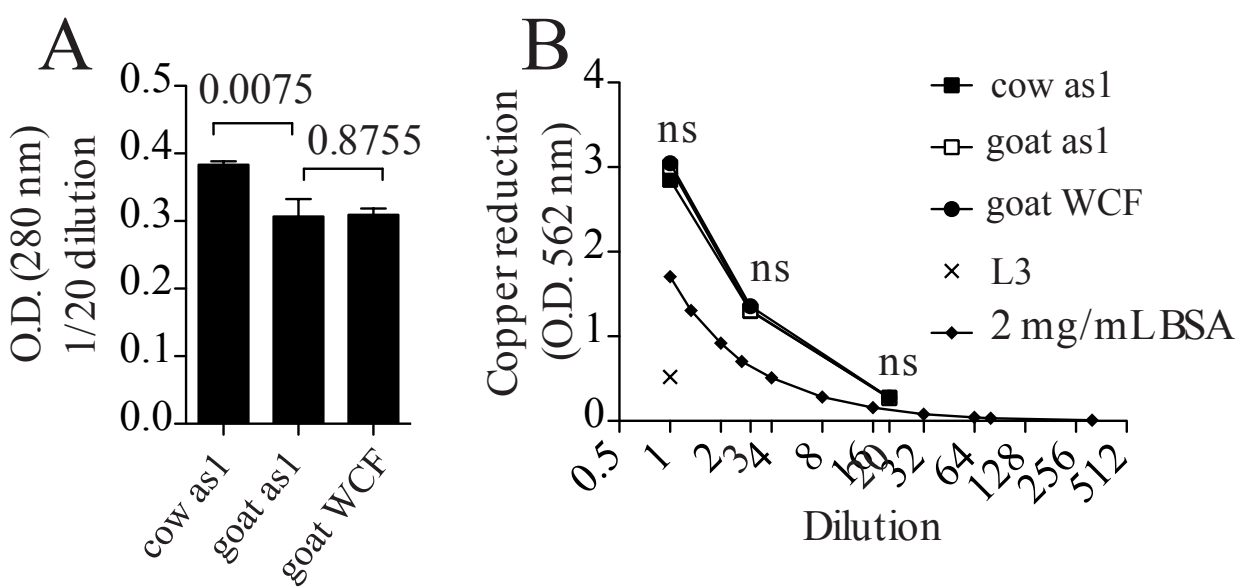

Figure 6.3: Comparison of cow and goat casein concentrations by BCA and nanodrop. Cow $\alpha$ s1-casein (cow as1), goat $\alpha$ s1 casein (goat as1) or goat whole casein (goat WCF) were dissolved in solution to $1 \mathrm{mg} / 90 \mu \mathrm{L}(11.111 \mathrm{mg} / \mathrm{mL})$. (A) After diluting samples $1 / 20$, proteins were compared for absorptive capacity by nanodrop $(280 \mathrm{~nm})$. (B) Serial dilutions of samples were compared to a standard $2 \mathrm{mg} / \mathrm{mL} \mathrm{BSA}$ solution for $\mathrm{Cu}^{2+}$ reduction based on the BCA reduction assay. Three 1/20 dilutions were prepared from the same stock for nanodrop; BCA data were in duplicate. 600 dead $i$ L3 $N$. brasiliensis larvae (L3) were also included in the assay (cross). Data are from a single experiment and were analysed by (A) Student's $t$-test, and exact pvalues are shown or (B) Two-way ANOVA with Bonferroni's post-test; non-significant (ns) $\mathrm{p}>0.05$. 

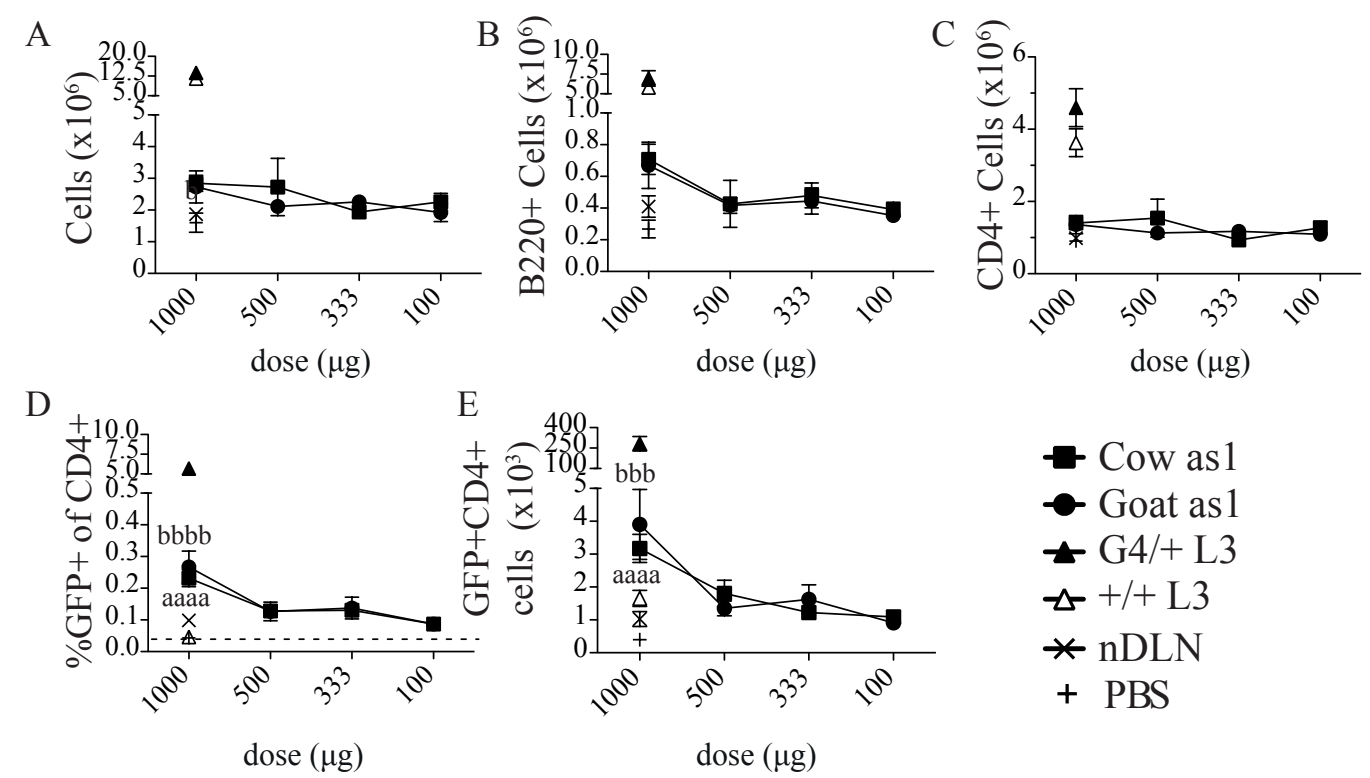

Figure 6.4: Similar Th2 response to cow and goat $\alpha$ s1-casein. Seven days after intradermal injection of $+/+$ (white triangles) or G4/+ (black triangles) mice with 600 dead $i$ L3 $N$. brasiliensis larvae, or cow (squares) or goat (circles) $\alpha$ s1-casein at the stated dose (in $\mu \mathrm{g}$ ), mice were euthanised and auricular cell infiltrates were profiled. Randomly selected non-draining lymph nodes including samples from all groups were used as baseline controls (crosses), and in one experiment PBS-immunized mice were also included (plus symbols). (A) The total number of cells was quantified by trypan blue exclusion and combined with flow cytometric analysis to determine the number of (B) B220+ cells, (C) CD4+ cells and the (D) proportion and (E) number of CD4+ $\mathrm{T}$ cells that expressed GFP. Symbols represent mean \pm SEM of data combined from three separate experiments, each using a different titrating dose; $1000 \mu \mathrm{g}$ Cow as1, $\mathrm{n}=11 ; 1000 \mu \mathrm{g}$ Goat as1 n=8; $500 \mu \mathrm{g}$ Cow as1 n=3; $500 \mu \mathrm{g}$ goat as1 n=5; $333 \mu \mathrm{g}$ cow or goat as $1 \mathrm{n}=2 ; 100 \mu \mathrm{g}$ cow or goat as $1 \mathrm{n}=5$; nDLN $\mathrm{n}=20 ; \operatorname{PBS} \mathrm{n}=4 ;+/+\mathrm{L} 3 \mathrm{n}=9$; G4/+ L3 n=11. Dose titrations were compared against the nDLN group using Twoway ANOVA with Bonferroni's post-test; aaaa $\mathrm{p}<0.0001$ Goat as1 vs. non-draining, b $\mathrm{p}<0.05$, bbb $\mathrm{p}<0.001$, bbbb $\mathrm{p}<0.0001$ cow as1 vs. non-draining. 


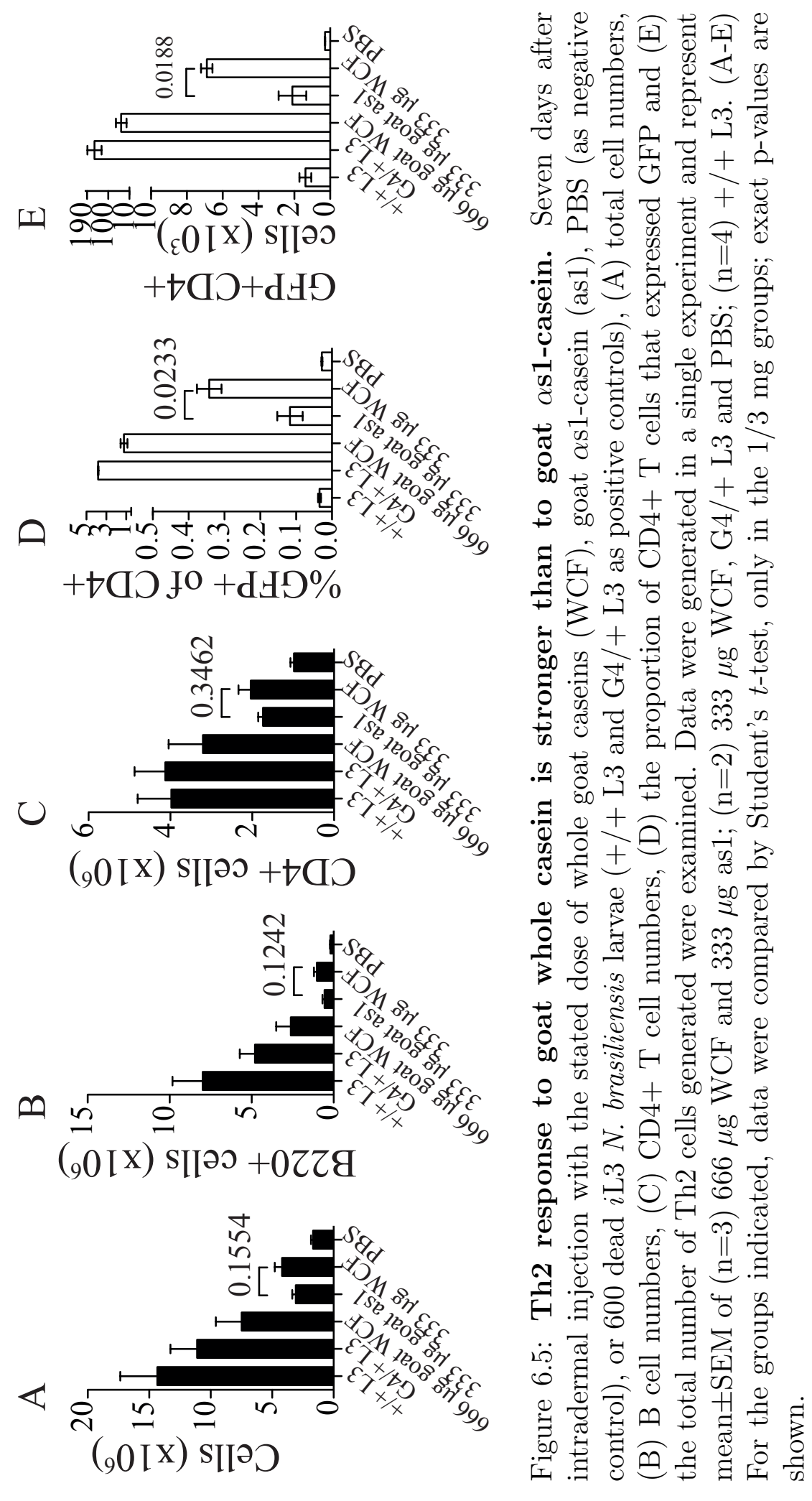

205 

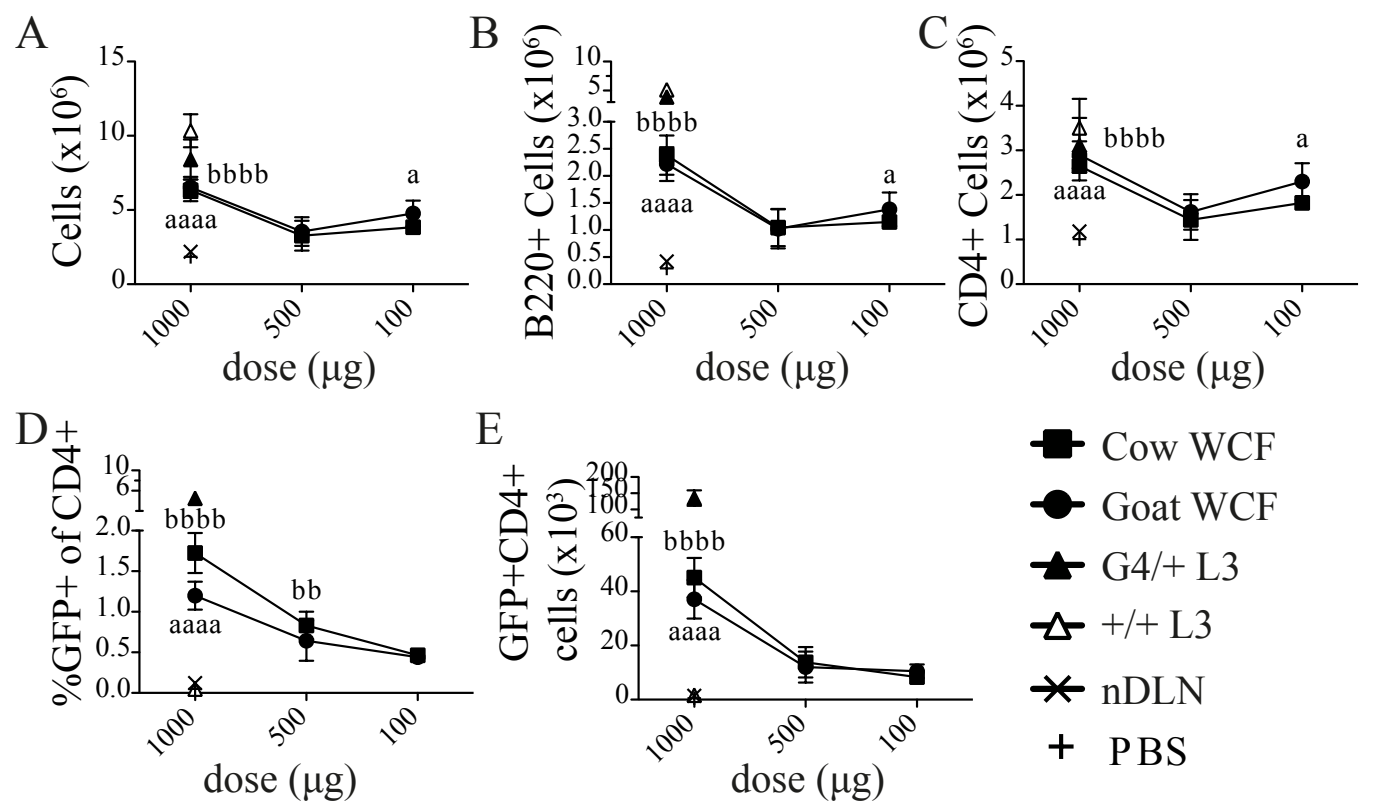

Figure 6.6: Th2 responses to cow and goat whole caseins are similar. Seven days after intradermal administration of $1 \mathrm{mg}$ (1000), $0.5 \mathrm{mg}$ (500) or $0.1 \mathrm{mg}$ (100) cow whole casein fractions (Cow WCF) or goat whole casein fractions (goat WCF), draining lymph nodes were examined for immune responses compared to non-draining $(\mathrm{nDLN})$ lymph nodes. $+/+(+/+\mathrm{L} 3)$ and G4/+ (G4/+ L3) mice immunized with 600 dead $i$ L3 $N$. brasiliensis larvae were used as positive controls. In one experiment a PBS (PBS) treated group was included as an additional negative control. (A) Total cell infiltrates, (B) B cell infiltrates, (C) CD4+ T cell infiltrates and (D) the proportion and (E) number of Th2 cells induced were recorded. Data points represent mean \pm SEM of $(n=6)$ mice for $N$. brasiliensis controls, $(n=14)$ non-draining nodes, $(\mathrm{n}=8)$ draining nodes at $1 \mathrm{mg}$ and $(\mathrm{n}=4)$ draining nodes at $0.5 \mathrm{mg}$ and $0.1 \mathrm{mg}$, combined from two experiments. a $\mathrm{p}<0.05$, aaaa $\mathrm{p}<0.0001$ goat caseins versus nondraining nodes, bb $\mathrm{p}<0.01$, bbbb $\mathrm{p}<0.0001$ cow caseins versus non-draining nodes by Two-way ANOVA with Bonferroni's post-test. 


\subsubsection{Examining the ability of goat milk to suppress Th2 re- sponses}

We used the pTh2 assay to test if goat milk can suppress Th2 responses induced by other allergens. Mice received $0.1 \mathrm{mg}$ HDM antigen in the presence or absence of 25 $\mu \mathrm{L}$ skim or whole goat milk, in a total volume of $30 \mu \mathrm{L}$ and seven days later lymph node cellularity was assessed. We observed increased Total, B cell and CD4+ T cell infiltration in HDM-immunized mice (Figure 6.7A-C). In terms of both proportion and number, the pTh2 response was strongest in mice immunised with HDM in the presence of whole goat milk (Figure 6.7D and E). Therefore, not only were goat milks unable to significantly suppress HDM-induced Th2 responses, the presence of whole goat milk increased the Th2 response.

Previous studies have examined the oral allergic response to milks. We next incorporated the physiological processing component of allergenicity into our investigations. Using the model developed by Brandt et al.[321], we examined the development of allergic diarrhoea in mice sensitised to OVA, then fed OVA dissolved in PBS, skim goat milk or whole goat milk. When mice were challenged perorally with milks alone, neither skim- nor whole-goat milk caused diarrhoea development in OVA-sensitised mice (Figure 6.7F). In contrast, when mice were challenged with $50 \mathrm{mg}$ OVA, all groups began to develop diarrhoea in 3-5 challenges, and disease penetrance reached $100 \%$ in all OVA-challenged groups, even those in which the OVA was dissolved in goat milk. Therefore, allergic diarrhoea in OVA-sensitized mice was neither induced nor inhibited by the presence of skim or whole goat milk, and there was no apparent difference of the response in the presence of either skim or whole goat milk. 


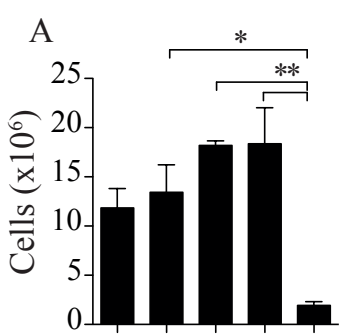

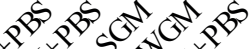

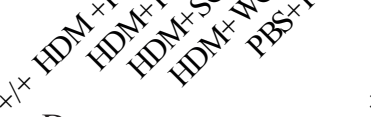

$\mathrm{D}$

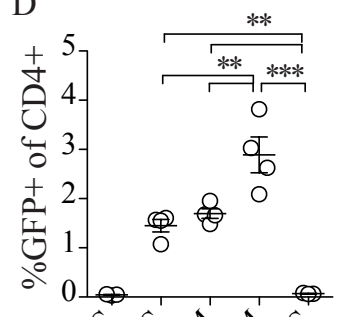

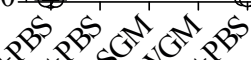

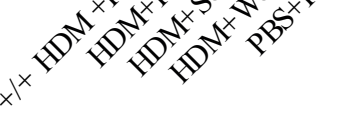

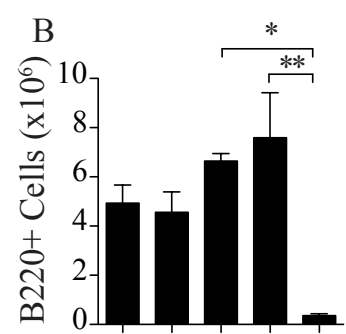

रु सु की से सु
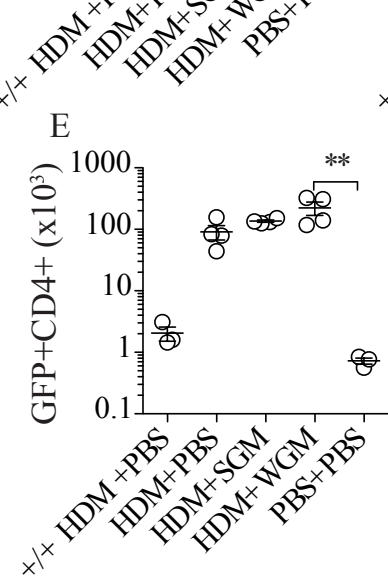

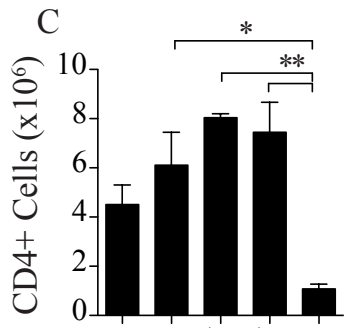

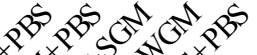

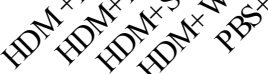

$\mathrm{F}$

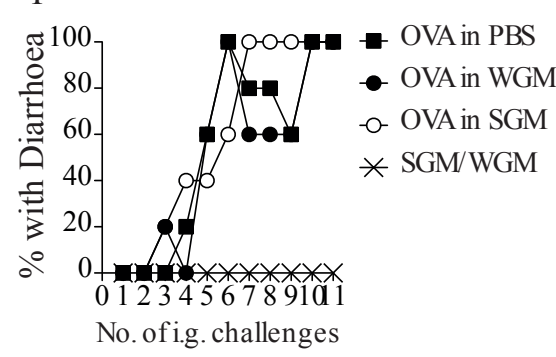

Figure 6.7: Neither skim nor whole goat milk prevent Th2 responses. (A-E) HDM was dissolved and then combined in a 5:25 ratio such that $30 \mathrm{uL}$ contained $100 \mathrm{ug} \mathrm{HDM}$ in $5 \mathrm{uL}$ PBS, $+25 \mu \mathrm{L}$ PBS, $25 \mu \mathrm{L}$ skim goat milk (SGM) or $25 \mu \mathrm{L}$ whole goat milk (WGM). A mock-challenged group was included to set the baseline (PBS+PBS). Seven days after injection of the stated solution, (A) Total cells were counted by trypan blue exclusion and flow cytometry proportions were used to backcalculate the number of (B) B cells, (C) CD4+ T cells, (D) the proportion of CD4+ T cells that expressed GFP and (E) the number of Th2 cells. Data shown are from one of two similar experiments. (D,E) Data points represent individual mice. (A-C) Bars show mean \pm SEM of these same mice. In the second experiment, $30 \mu \mathrm{L}$ PBS, SGM or WGM were administered to the ear 36 hours prior to HDM immunization and gave similar results. (F) OVA-sensitized mice ( $\mathrm{n}=5 /$ group) were i.g. challenged with $50 \mathrm{mg}$ OVA in PBS, SGM or WGM. Control OVA-sensitised mice received 250 $\mu \mathrm{L}$ skim goat milk (SGM; $\mathrm{n}=2$ ) or whole goat milk (WGM; $\mathrm{n}=3$ ) alone during i.g. challenge. Oral challenge data were generated in a single experiment. (A-E) Data were compared using One-way ANOVA with Tukey's post-test; ${ }^{*} \mathrm{p}<0.05,{ }^{*} \mathrm{p}<0.01$, $* * * \mathrm{p}<0.001$. 


\subsubsection{The oral response to milk allergens}

Homologous goat and cow milk allergens induced similar Th2 responses, but the pTh2 assay does not examine the full allergic process. Using the model developed by Brandt et al.[37], we aimed to establish whether oral challenge with goat BLG could initiate the intestinal mastocytosis associated with oral potentiation.

Mice were sensitised twice, two weeks apart with $50 \mu \mathrm{g}$ goat BLG with alum, and then subsequently fed with $50 \mathrm{mg}$ goat BLG, $50 \mathrm{mg}$ OVA, or PBS. A control group of mice was mock-sensitized, and then fed with $50 \mathrm{mg}$ goat BLG. When we examined IgE levels, total IgE increased in sensitized mice fed goat BLG (Figure 6.8A). Feeding goat BLG-sensitised mice with PBS or OVA did not increase the Total IgE above starting titres (Figure 6.8A). Similarly, mock-sensitised mice fed with goat BLG had lower IgE than the goat BLG-sensitized, goat BLG-fed group at the experimental endpoint (Figure 6.8A). We examined antibody binding to goat BLG and observed minor elevations in the BLG-fed group over other groups for both polyclonal Igs and IgG1 (Figure 6.8C and E). When we examined these mice for IgG1 binding to cow BLG, we similarly observed elevations over the other groups (Figure 6.8D and F). These data indicate that oral challenge with goat BLG increased both goat and cow BLG-specific antibody titres. Last, we examined serum mMCP-1 levels in these mice. The only group in which we detected increased mMCP-1 levels was the goat BLG-sensitised, goat BLG-fed group (Figure 6.8B) with corollary increases in jejunal mast cell frequencies (Appendix Figure 8.7). From these data, we conclude that oral potentiation was observed in goat-BLG fed mice. Therefore, goat BLG can both sensitize and initiate the oral allergic process.

To compare the allergenicity of cow and goat BLG, we used the contracted experimental model in $i F A B P p$-IL-9 transgenic mice. These mice were sensitised to OVA, goat BLG, cow BLG, BSA or were mock-sensitised with PBS. Seven days later, mice were orally gavaged with $50 \mathrm{mg}$ of the sensitising allergen nine times. Mice were bled after the ninth challenge, and sera were analysed for parameters associated with the intestinal allergic response: $\mathrm{mMCP}-1, \operatorname{IgE}$ and IgG1 production. Further, because we previously observed increases in IgG1-ASC numbers in mice sensitised and orally-primed with OVA, when we killed the mice four days after their final gavage 
challenge, we also examined the number of antigen-specific ASCs in spleens of all groups of mice.

Before discussing the results of this experiment, it is important to note that in the early challenges, we observed diarrhoea development in 75 percent of cow BLG-fed mice after the third challenge (images are shown in the appendices). We attempted to bleed mice after the third challenge, but blood pressure was low in the cow BLGfed group, and we were unable to attain sufficient blood to isolate plasma. In the preparation of this cow BLG, $2 \%$ salt $(\mathrm{NaCl})$ was added to help solubilise it, so to remove any confounding this may have had, cow BLG was subsequently dialysed prior to gavage, and thereafter diarrhoea was no longer observed in cow BLG-fed mice. However, in a repeat experiment, neither sensitised nor unsensitised cow BLGfed mice developed diarrhoea using the same mass of undialysed cow BLG; as such we were unable to reproduce the diarrhoea phenotype, nor were we able to determine whether it was IgE- and mast cell dependent. Diarrhoea developed in one of three OVA-fed mice, and in none of the BSA- or goat BLG-fed mice. Therefore, we have limited our evaluation of the results to the production of mMCP-1 and cross-reactive IgG1 detection.

When we measured mMCP-1 levels in the OVA, goat BLG, cow BLG and PBS-fed groups, we observed increases in all three groups of allergen-challenged mice over PBS-fed mice (Figure 6.9A). While OVA, goat BLG and cow BLG groups were all elevated over the mock-sensitized group, the OVA-fed group was most significantly elevated, but not significantly over the two BLG groups (Figure 6.9A). The level of Total IgE at the experimental endpoint was significantly elevated in the cow BLG group over mock-sensitised, PBS-fed mice (Figure 6.9B), and similar trends for the other groups were observed.

We also observed that ASC formation was similar for cow and goat-fed groups (Figure 6.9C-F), and also correlated approximately with the antigen-specific IgG1 titres that developed (Figure 6.10A-D). When we examined IgG1-binding to the different proteins, cow BLG- and goat BLG-specific antibodies were detected in both cow and goat BLG sensitized mice, but not OVA-sensitized mice (Figure 6.10B, C, F, G). Somewhat intriguingly, cow BLG-fed iFABPp-IL-9 transgenic mice had higher cow BLG-specific 
IgG1, and polyclonal Igs than goat BLG-fed mice at the 1/10000 dilution (Figure 6.10B and F). This same relationship was, however, not observed in the spot assay (Figure 6.9D). Such significant cross-reactivity was not observed between BLG and either OVA or BSA, in any of polyclonal, antigen-specific IgG1, or spot assays (Figure $6.9 \mathrm{C}-\mathrm{F}$ and $6.10 \mathrm{~A}-\mathrm{H})$. Taken together, these data may indicate that oral challenge with both cow and goat BLG causes oral potentiation; both homologues drive similar magnitude increases in $\mathrm{mMCP}-1$ production but may differentially affect the $\mathrm{B}$ cell response. However, further investigation is required to definitively determine whether the ability of the two BLGs to drive oral potentiation differ.

Taken together, these preliminary findings suggest that the sensitising and responseevoking capacity of goat BLG and cow BLG were similar, but indicated that cow-BLG feeding increased BLG-specific IgG1 titres. These studies will benefit from replication in the future. At this stage, given the similar elevations in the mMCP-1 response, from these data we conclude that the response-evoking capacity of BLG from cow or goat milk was similar. 


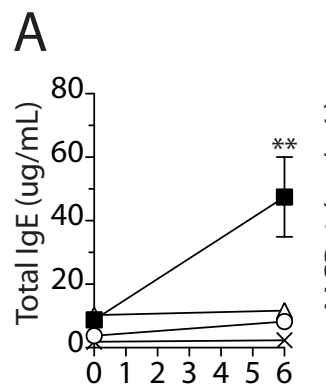

B

No. of i.g. challenges
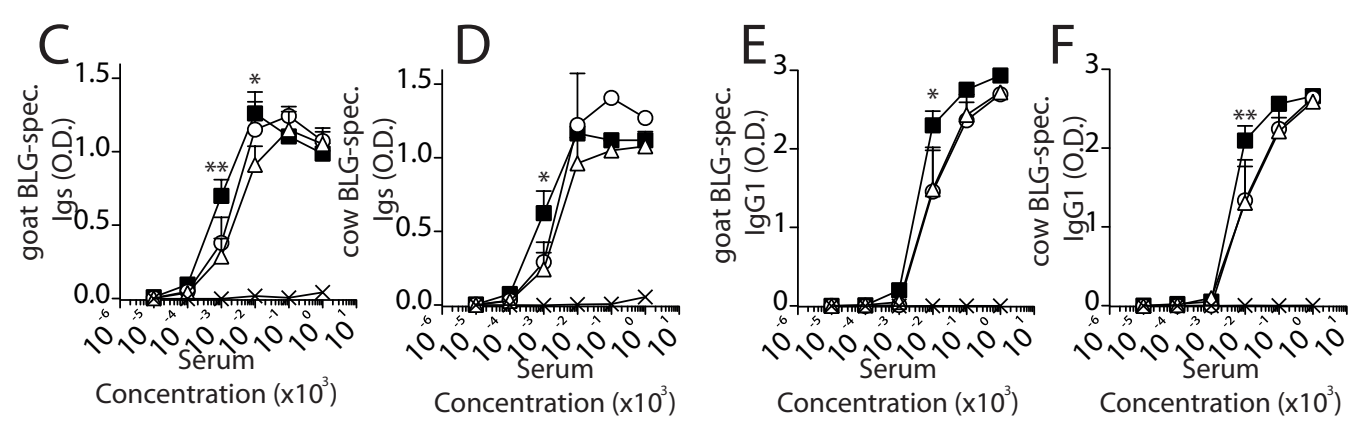

Figure 6.8: Goat $\beta$-lactoglobulin can induce intestinal Th2 responses. Groups of ( $\mathrm{n}=3$ /group) mice were sensitized ip to goat BLG with $50 \mu \mathrm{g}$ goat BLG plus $1 \mathrm{mg}$ alum (goat BLG/) or mock-sensitized with PBS plus $1 \mathrm{mg}$ alum (PBS/) and then orally challenged with goat BLG (/goat BPG), OVA (/OVA) or PBS (/PBS) as indicated. Mice were bled for (A) Total IgE before any and after six challenges. (B) mMCP-1 was analysed in sera after the $6^{\text {th }}$ challenge. (C) goat BLG-specific polyclonal Igs, (D) cow BLG-specific polyclonal Igs, (E) goat BLG-specific IgG1 and (F) cow BLG-specific IgG1 were analysed by ELISA. (B) Data points represent individual mice or $(\mathrm{A}, \mathrm{C}-\mathrm{F})$ mean $\pm \mathrm{SEM}$ of these groups. Data were generated in a single experiment and were analysed using (B) One-way ANOVA with Tukey's post-test or (A, C-F) Two-way ANOVA with Bonferroni's post-test; $\mathrm{p}<0.05,{ }^{* *} \mathrm{p}<0.01$. mMCP-1 data were log-transformed prior to analysis. 


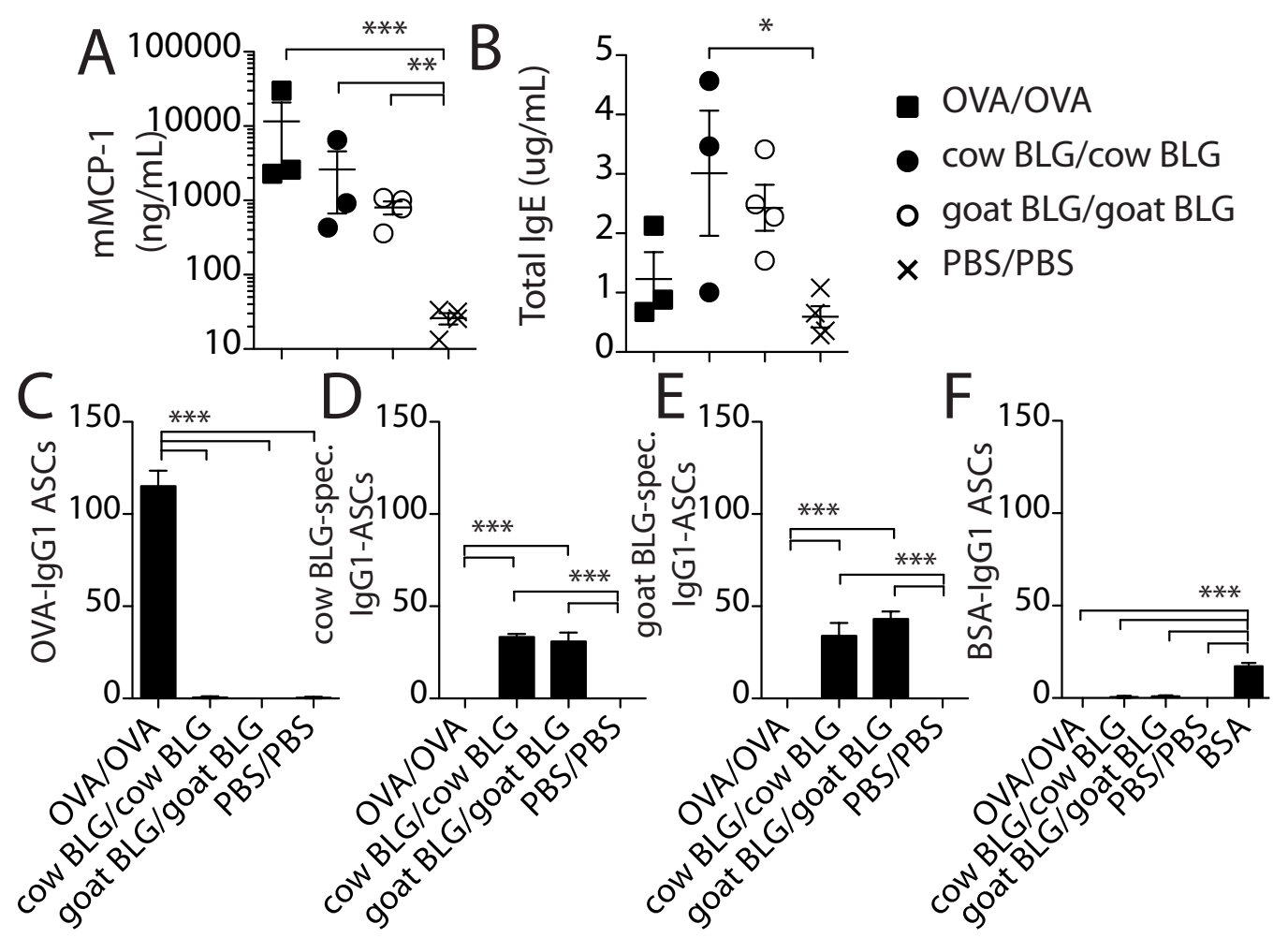

Figure 6.9: mMCP-1 levels are elevated in orally-challenged sensitized mice. iFABP $p$-IL-9 mice were i.p. sensitized, and seven days later began to receive i.g. challenges with: i) OVA (OVA/OVA), ii) cow BLG (cow BLG/cow BLG), iii) goat BLG (goat BLG/goat BLG), iv) PBS (PBS/PBS) or v) BSA (BSA). i.p. sensitizations used $50 \mu \mathrm{g}$ of the indicated allergen; i.g. challenges used $50 \mathrm{mg}$ of that same allergen and were given every second day, three times a week for three weeks. Two hours after the $9^{\text {th }}$ i.g. challenge, mice were bled and (A) serum mMCP-1 and (B) Total IgE levels were evaluated. (C-F) Antigen-specific IgG1 spot assays were performed for each allergen. FBS was used to block plates so BSA group was not analysed in the other spot assays. Data were generated in a single experiment. (A,B) Data points represent individual mice, $(\mathrm{C}-\mathrm{F})$ bars represent mean $\pm \mathrm{SEM}$ of these same groups. Data were compared using One-way ANOVA with Tukey's post-test; ${ }^{*} \mathrm{p}<0.05$, ${ }^{* *} \mathrm{p}<0.01,{ }^{* * *} \mathrm{p}<0.001$. mMCP-1 data were log-transformed prior to analysis. 

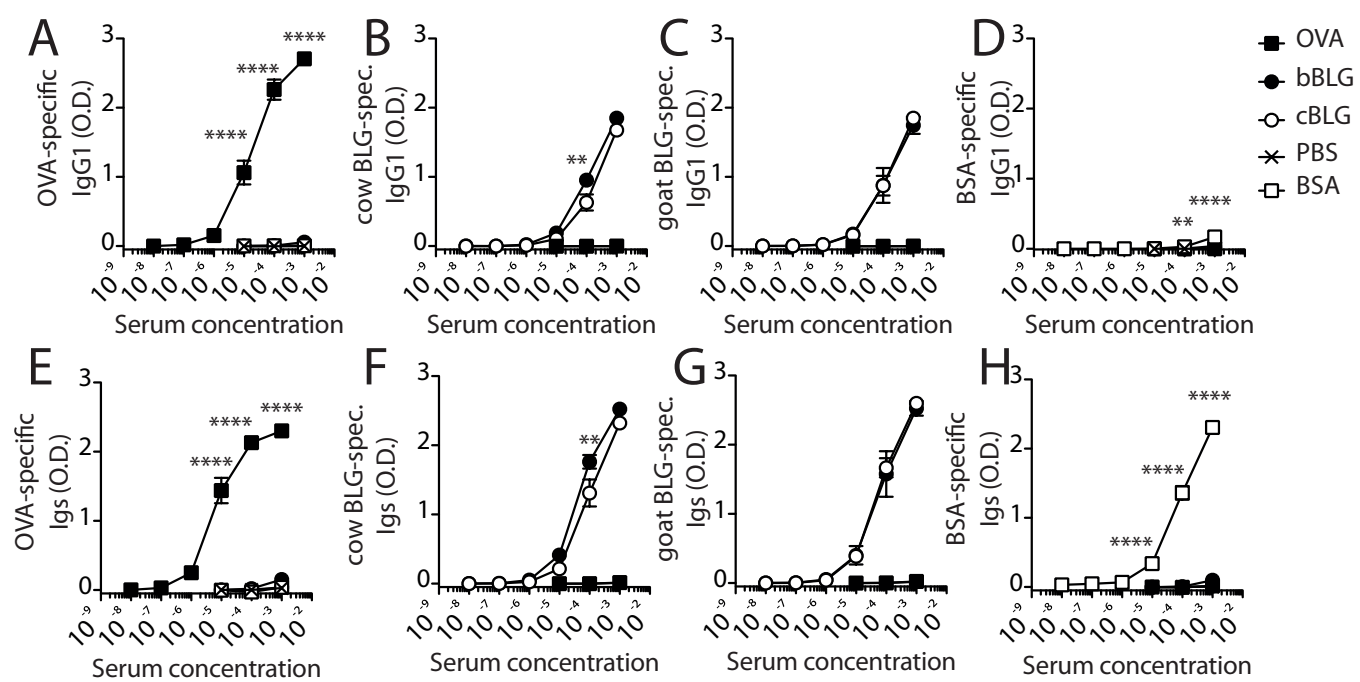

Figure 6.10: Cross-reactivity observed between cow and goat $\beta$-lactoglobulin but not other allergens in $\boldsymbol{i F A B P} \boldsymbol{p}$-IL-9 mice. Sera from mice described in Figure 6.9 were analysed for (A) OVA-specific IgG1, (B) cow BLG-specific IgG1, (C) goat BLG-specific IgG1, (D) BSA-specific IgG1, (E) OVA-specific polyclonaly Igs, (F) cow BLG-specific polyclonal Igs, (G) goat BLG-specific polyclonal Igs or (H) BSAspecific polyclonal Igs. (A, D, E, H) Represent significance against all other groups, in $(\mathrm{B}, \mathrm{C}, \mathrm{F}, \mathrm{G})$ statistical differences are shown for cow versus goat $\beta$-lactoglobulin groups. Data were generated in a single experiment and compared using Two-way repeated measures ANOVA with Bonferroni's post-test; ${ }^{* *} \mathrm{p}<0.01,{ }^{* * * *} \mathrm{p}<0.0001$. 


\subsection{Discussion}

In this chapter we sought to establish the components of milk that influence its allergenicity. We used the pTh2 assay to compare homologous cow and goat milk proteins. We examined BLG, $\alpha$ s1-casein, and also the whole casein fraction. We further investigated the anti-allergic properties of skim and whole goat milks, and the oral potentiation that follows oral challenge with goat or cow BLG. In the pTh2 assays, we observed similar, dose-dependent Th2 cell development in response to primary cow and goat BLG, $\alpha$ s1 or whole casein fraction immunisation. These findings indicated that the intrinsic allergenicity of these goat and cow milk allergens was similar. We also found that goat milk itself did not appear to suppress Th2 responses. Our oral challenge studies identified that goat BLG can induce antigen-specific mast cell responses in the intestine, and preliminary data indicated that oral challenge with OVA, cow or goat BLG caused oral potentiation in $i F A B P p$-IL-9 transgenic mice. In our data we observed no significant difference in the level of potentiation invoked by either cow or goat BLG.

\subsubsection{Evidence for differential allergenicity of goat and cow milk}

Goat and cow milks have been compared previously. Numerous clinical studies have investigated their allergenicity. Bellioni-Businco et al.[192] observed that a significant proportion of cow milk allergic individuals were also allergic to goat milk[192]. AhLeung et al. presented evidence of a number of individuals sensitized to goat milk, but not cow milk[221]. Significant cross-reactivity between milk proteins from different ruminant species is usually observed[192, 247]. The IgE binding to cow $\alpha$ s1-casein is also frequently very strong[182]. Ballabio et al. recently observed that goat milk with low or no $\alpha$ s1-casein induces minimal skin prick positivity in some cow milk sensitized individuals[236]. There is evidence of cross-reactivity, however, there is also clinical evidence of cases in which the milks are antigenically distinct.

In experimental systems, comparisons have also been made between cow and goat milk. McLaughlan et al.[238] ran experiments in guinea pigs. Guinea pigs that were 
given ad libitum access to cow milk became sensitized and demonstrated frequent fatal anaphylaxis when i.v.-challenged with cow BLG or whole caseins[238]. Guinea pigs sensitized to goat milk in the same manner also succumbed to the i.v. challenge[238]. However, it was found that if the milks were boiled prior to feeding, the incidence of fatal i.v.-induced anaphylaxis was reduced, and reduced more markedly for boiled goat milk[238]. More recently, in a contracted version of the same experiment, the incidence of fatal anaphylaxis was less frequent in goat milk fed mice[141]. Bevilacqua et al.[249] observed that when guinea pigs were senstized to whole goat or whole cows milk, the antibody response was directed against BLG, and milk that had low $\alpha$ s1-casein content caused less intestinal anaphylaxis[249]; goat milk that had high $\alpha$ s1-casein caused similar disease severity to cow milk[249]. Similarly, in mice, LaraVilloslada et al.[234] demonstrated that mice sensitized orally and challenged i.v. with skim cow milk released more plasma histamine, had elevated IgG1 titres, had more severe anaphylactic symptoms and a skewed Th2 cytokine response profile compared to skim goat milk treated mice[234]. Therefore, there is experimental evidence that links the allergenicity of goat milk to its $\alpha$ s1-casein content.

\subsection{2 $\alpha$ s1-casein as an allergen}

Given the evidence in the literature that indicates that $\alpha$ s1-casein is a dominant allergen, the comparatively low pTh2 response to $\alpha$ s1-casein was surprising. The $\alpha$ s1-casein content of milk has many times been associated with its allergenicity in experimental systems[141, 232, 234, 249, 250], and we expected to observe significant differences. However, not only was there no apparent difference in Th2 development between cow and goat $\alpha$ s1-casein, both were also poorly immunogenic. In response to a $1 \mathrm{mg}$ dose, we observed that the total immune cell infiltrate increased by only twofold, and the CD4+ T cell population did not observably expand (Figure 6.4).

These findings may indicate that $\alpha$ s1-casein influences the allergenicity of milk through interaction with other milk components. Caseins form micelles, and $\alpha$ s1-casein influences the structure and size of the micelle[230]. Johansson et al.[474] argue that the biasing of monoclonal antibodies that develop against caseins toward $\kappa$ casein, could reflect an important role for the supramolecular structure of the casein micelle in its 
immunogenicity[474]. In our studies, we observed that the whole casein fraction was more immunogenic than $\alpha$ s1-casein in isolation (Figure 6.5), which may support this finding. Processing of milks can alter their basic physical properties. Heat-treating milks can induce covalent linkages between BLG peptides and $\kappa$ casein[475]. The combined effects of caseins and BLG may be important for the allergenicity of milks, and have been shown to be interrelated in the past[249, 250]. More recently, it has been shown that milk with high $\alpha$ s1-casein content has larger fat globule diameter[476]. In our studies, we observed that whole milk increased the pTh2 response to HDM to a greater extent than did skim milk (Figure 6.7); we used low $\alpha$ s1-casein milk in these studies, but it is possible that higher fat content, or globule size could have increased the response even more. Although we saw augmented responses to HDM in the presence of whole milk compared with skim milk in the pTh2 model, we did not see this same effect in the oral challenge model (Figure 6.7). Side-by-side comparisons of cow and goat milk fats on the pTh2 response may reveal some additional $\alpha$ s1-casein-associated aspects that do not relate to its intrinsic allergenicity or autoadjuvanticity.

In the appropriate circumstances, $\alpha$ s1-casein can induce Th2 responses in isolation. Recently, $\alpha$ s1-casein from goat milk was shown to orally potentiate sensitized mice, because mMCP-1 levels increase after $\alpha$ s1-casein feeding[394]. In this study, $\alpha$ s1casein also triggered increased IgE production, and higher IL-4 and IL-10 release by splenocytes cultured with $\alpha$ s1-casein[394]. This report indicates that $\alpha$ s1-casein can act as an allergen in mice. One difference is that this system employed a presensitization, where mice were administered $\alpha$ s1 casein i.p. with alum adjuvant prior to i.g. challenge[394]. In our studies, we did observe some Th2 cell development in response to $\alpha$ s1-casein (Figure 6.4), but the addition of an exogenous adjuvant is likely to amplify the immune response. These findings may indicate that $\alpha$ s1casein has low auto-adjuvanticity, but in the presence of an adjuvant can become a significant allergen. Natural adjuvants that contribute to sensitization could come in the form of staphylococcal superantigens[477], or alternatively, may not be required for sensitization if the allergen was exposed to a mucosal surface (e.g., the lung; [136]). Additionally, as there are homology differences between cow and human milk proteins, the absence of an observable effect in the mouse may relate to differences in protein 
homology between cow, goat and mouse milk proteins, in contrast to cow, goat and human milk proteins. Further studies will be required to determine the comparative allergenicity of goat $\alpha$ s1-casein to cow $\alpha$ s1-casein in oral challenge models, and test alternative means by which $\alpha$ s1-casein could sensitize.

\subsubsection{The allergenicity of BLG}

We investigated BLG allergenicity using the pTh2 assay and in the oral challenge model. In our pTh2 assays, we observed equivalent allergenicity of cow and goat BLG. Both proteins induced 15-100-fold increases in Th2 cell development when injected at 1 mg, which indicated that the intact allergens were equivalently allergenic (Figure 6.2). However, evidence in the literature encouraged us to investigate the response in an oral challenge model. Goat BLG was shown in the past to induce intestinal responses in guinea pigs[249]. Cow BLG has been demonstrated to induce mast cell degranulation in oral challenge models[263]. There is also evidence in the literature that while they are both stable proteins, the digestibility of the two BLGs differ: $50-80 \%$ of goat BLG is degraded, compared to only $20 \%$ of cow BLG when subjected to sequential digestion in human gastric- and then duodenal juices[185, 478]. We considered that if goat milk was more digestibile and more rapidly degraded in the murine gastrointestinal tract the amount of intact antigen available to drive the inflammatory process may be reduced and result in a similar four-fold differential in their ability to orally prime and induce mMCP-1 production.

Earlier studies undertaken at AgResearch identified that goat $\beta$-lactoglobulin can drive oral potentiation (Ali Hodgkinson, personal communication). However, mast cells can produce mMCP-1 in an IgE-independent manner[479], so it was important to confirm that the oral response only occurs in response to sensitizing antigen. In our oral challenge studies, we observed that six oral challenges with $50 \mathrm{mg}$ goat BLG was sufficient to orally potentiate, because mMCP-1 titres increased from $\approx 20 \mathrm{ng} / \mathrm{mL}$ to $\approx 5000 \mathrm{ng} / \mathrm{mL}$ in orally-fed mice (Figure 6.8 ). We also observed elevated $\operatorname{IgE}$ titres, only in sensitized, allergen-fed mice. Further, feeding goat BLG-sensitized mice with OVA did not increase mMCP-1 release above mock-challenged and/or sensitized controls. Therefore, oral potentiation induced by goat BLG feeding required sensitization 
and was goat BLG specific.

In the literature, the mast cell response correlates with the development and severity of oral allergic responses[36, 38]. In our studies in the previous chapter (Chapter 5), we confirmed that the intestinal mast cell response was a CD4 $+\mathrm{T}$ cell-dependent event. Therefore mMCP-1 production can be used to reflect the allergenicity of an orally administered protein.

In a preliminary investigation, we used the $i F A B P p$-IL-9 transgenic mice to compare the allergenicity of OVA, cow and goat BLG. We observed that oral potentiation occurred when any of OVA, cow or goat BLG were used, because mMCP-1 titres were elevated compared to PBS-fed mice (Figure 6.9). In terms of their comparative allergenicity, the low power of the experiment, and unexpected, but unreproducible, diarrhoea development in cow BLG-sensitized mice prevents us from making definitive conclusions. However, there were trends indicating that OVA induced the most mMCP-1 release, generated the most frequent splenic IgG1-ASCs, and had high serum IgG1 titres (Figures 6.9 and 6.10). Similarly, while mMCP-1 levels induced by cow and goat BLG were similar, the cow BLG-fed group demonstrated increases in serum cow BLG-specific antibodies that were not observed in the goat-fed group (See Appendices). Additionally, early in the response, we observed diarrhoea and severe plasmavolaemia in the cow BLG-fed group. We were unable to repeat this finding, and further were unable to link this to the allergic process because we could not bleed these mice post-challenge. However, it does indicate that oral processing may be an important feature of the differential allergenicity of cow and goat BLG that could be revealed in follow-up studies using larger experiments.

\subsubsection{Future investigations that could clarify goat milk pro- tein allergenicity}

To accurately clarify the role of oral processing in milk protein allergenicity, there are several potential experiments that would be useful. One of the most informative tests will be to directly assess antibody cross-reactivity in cow and goat BLG-fed mice, which could be done using antigen inhibition tests[480]. Lung challenge models 
and alternate oral challenge models have been employed using cow BLG[139, 458]. Cytokine levels produced by splenocytes, intestinal cells or mesenteric lymph node cells have been compared for other allergens[57, 394]. Examining these responses may reveal differences in the oral potentiation response, and clarify whether the trend we observe reflects a true difference, or relates to experimental variation.

The pTh2 studies could also be expanded. Examining the local Th2-mediated B cell response may indicate additional aspects of protein allergenicity that are not revealed by measuring Th2 development. While we observe IgG1 switching in the pTh2 assay, and this correlated with the Th2 response (Chapter 4), distinct mechanisms influence IgE class switching[279, 306, 445, 481]. Specifically, while both IgE and IgG1 class switching are both controlled by TFH cells and sensitive to IL-4, they are reciprocally affected by IL-21[440-443, 482]. Therefore, examining IgE switching may be additionally informative. While beyond the sensitivity of most current tools, examining IgE switching may be possible in a modified pTh2 assay using recently developed IgE-reporter mice[444, 445].

In the course of our studies, we have found no difference in the intrinsic allergenicity, as defined by primary Th2 cell induction, for BLG, $\alpha \mathrm{s} 1$ or whole casein fractions isolated from cow or goat milks. Future work that analyses milk proteins in isolation, milk as a complete food and as its casein and whey fractions will have the potential to clarify the differences in cow and goat milk that render them differentially allergenic in experimental contexts.

\subsection{Summary}

Homologous milk allergens derived from cow and goat milk induced similar pTh2 responses to each other. $\alpha$ s1-casein was shown to have low allergenicity in isolation. The presence of milk fat increased the pTh2 response but did not expedite disease in the oral challenge model. Goat milk did not demonstrate strong anti-allergic properties in the models tested. Further investigation of how oral processing influences milk protein allergenicity is warranted. 


\section{Chapter 7}

\section{General Discussion}

\subsection{Summary of findings}

During the course of this work we uncovered some novel aspects of Th2 cell development and function. In Chapter 3, we determined that biallelic IL-4 production occurs more frequently than is predicted for a monoallelic, stochastic gene expression event. In Chapter 4, we identified IL-4-independent and -dependent phases of Th2 cell development, and also found that the biallelic IL-4-producing potential is important for allergen-specific Th2 immunity. In Chapter 5, we uncovered an important role for IL-3, and established the causal role of CD4+ T cells in allergen-induced intestinal mastocytosis. We further determined that this function of CD4+ T cells was required additional to their contribution to IgE production. Our final results chapter defined the Th2-inducing capacity of goat and cow milk components. It additionally provided preliminary information about the ability of goat BLG to induce intestinal mast cell activity. The findings of this thesis reaffirm the central role of CD4+ T cells in IgE-mediated allergic disease.

\subsubsection{Checkpoints along the road to allergy}

The inter-related aspects of Th2 immunity investigated in this project allow us to draw a more comprehensive picture of the experimental allergic process that ultimately results in diarrhoea. We identified numerous points of potential disease intervention in mice that warrant investigation in clinical settings based on current knowledge and the findings of this thesis. These concepts are summarised in figure 7.1. Taken as a whole, we consider that there are multiple points for disease intervention, however, the further along the process, the more difficult disease will become to treat. 

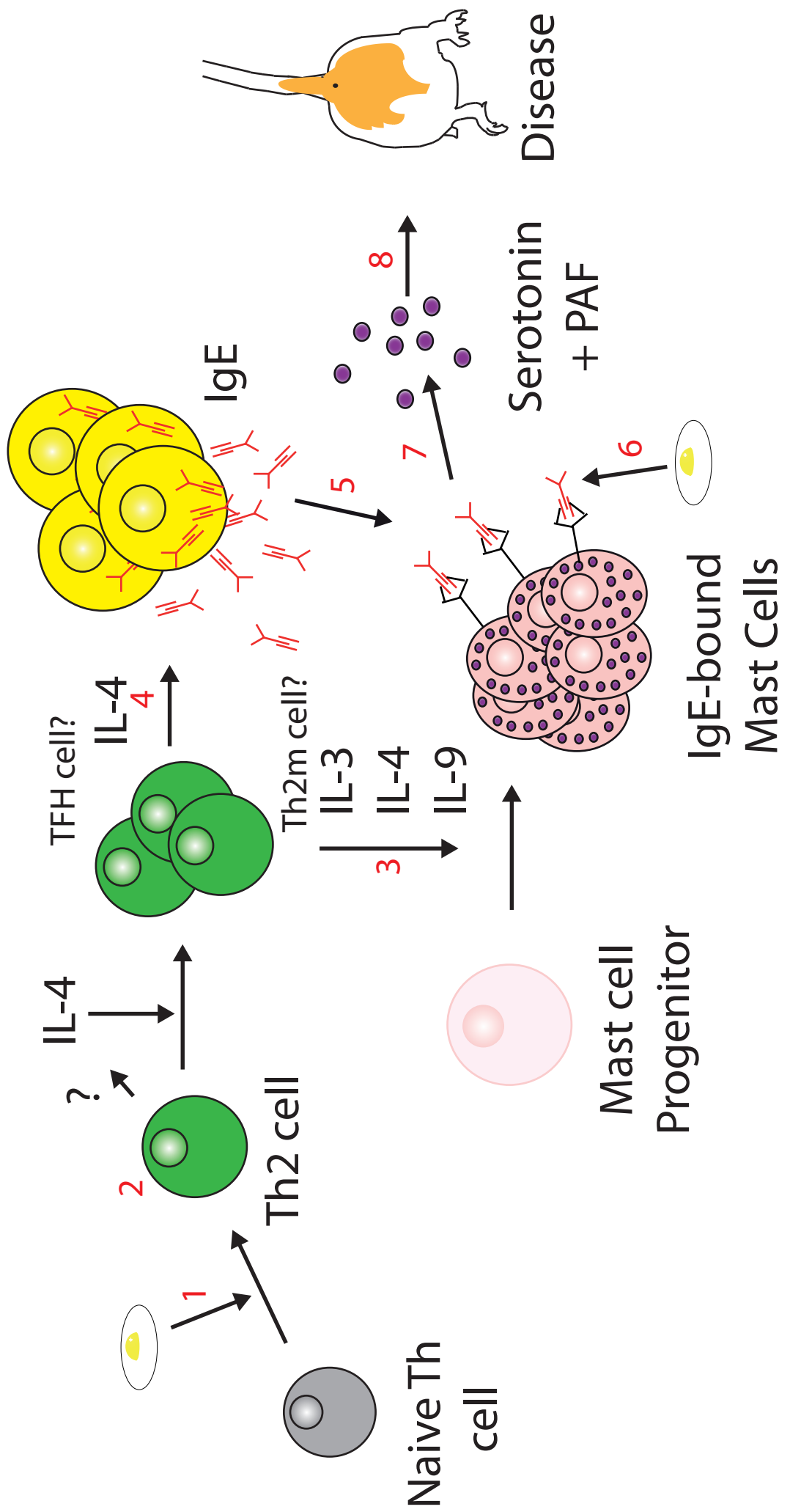

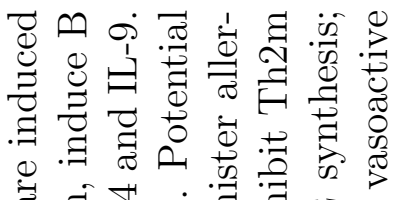

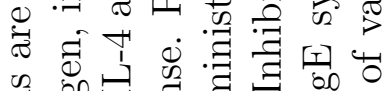
웧 0 娄 + 岁岑. $\overrightarrow{0}: \begin{gathered}0 \\ \mathrm{c}\end{gathered}$ ช

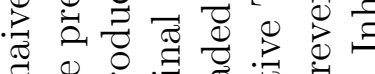

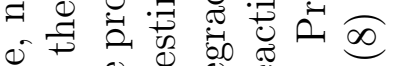

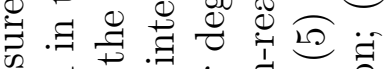
के fi $\overrightarrow{0}$ ॠ

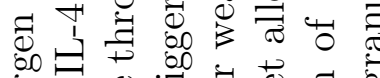
苟㟔.

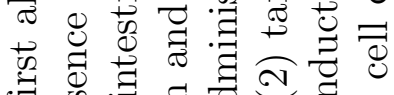

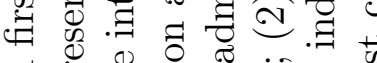

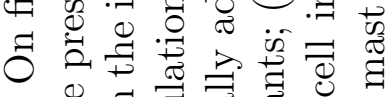

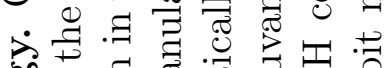
。․ㅠ. ส च

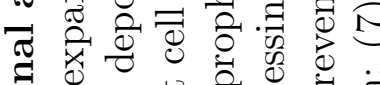

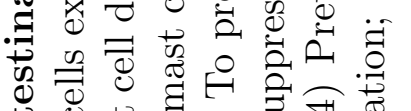

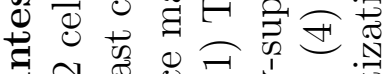

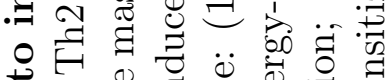
○o $\begin{gathered}0 \\ 0\end{gathered}$

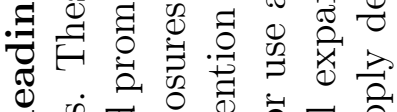

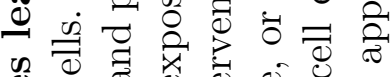

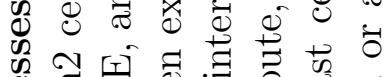

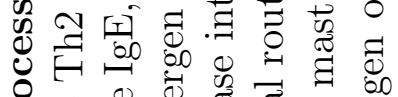

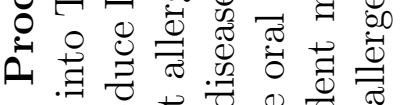
$\because \overrightarrow{0}$ oे

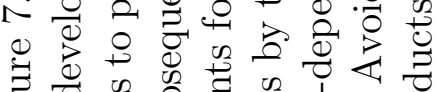

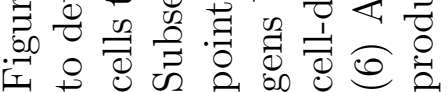




\subsubsection{Future research directions}

\section{Evaluating Biallelic Th2 cell function in vivo}

In this thesis, we examined Th2 cell development and function, largely using IL-4 haplo-insufficient, or IL-4-deficient reporter mice. We infer information about the regulation of IL-4 production from our data, namely that biallelic IL-4 production is more frequent than is expected based on the proposed model[401]. Determining whether this is important for specific aspects of allergic Th2 cell function, and whether this truly occurs in wild-type mice (and humans) in vivo, requires further investigation. The presence of biallelic IL-4 production in wild-type cells could be evaluated at the transcriptional level using single cell PCR analysis from in vivo-generated Th2 cells with distinguishable, but wild-type IL-4 alleles[389]. Additionally, the specific involvement of biallelic IL-4 production in Th2 immunity could be examined at a functional level. Deletion of an IL-4-silencer site results in non-specific IL-4 production[380], which could override the potential imbalance in the absolute concentration of IL-4 in IL-4 heterozygous mice. These mice would then be deficient only in biallelic IL-4 production. Examining IgG1 class-switching, the generation of IgE, and the development of allergic diarrhoea could make some headway into the role of biallelic IL-4 producing Th2 cells in the allergic immune process.

\section{Establishing the functional or physical divergence of Th2m and TFH cells}

The data in the oral allergen challenge model provides evidence for the existence of Th2m cells. However, it is unknown whether Th2m cells are a separate population from IgE-inducing cells. In addition to IgE production at systemic sites[444], antibody can be produced in high amounts in local tissues. Specifically, Kweon et al. identify IgE-producing B cells in the colonic intestinal lamina propria of orally-sensitized mice[58]. In this case, it is plausible that Th2m cells are also the cells involved in the amplification of systemic IgE following oral exposure, and contribute to both processes simultaneously. Determining the existence of divergent, or overlapping Th2 
cell functions in the intestinal allergic process may indicate whether targeting multiple CD4 $+\mathrm{T}$ cell populations will be required for effective disease intervention. Additionally, establishing the importance of IL-3 in other oral allergen-challenge models, and eventually in clinical contexts will clarify the overall involvement of IL-3 in the intestinal allergic process. Simple experiments could evaluate IL-3-deficient mice in the cholera toxin model[58, 140, 483], the superantigen oral allergic disease model[456], or use IL-3R-deficient mice to validate our findings in the model we used[113]. For clinical application, initial animal experiments could also examine whether anti-IL-3 antibodies can inhibit disease development in mice.

\section{Targeting adaptive immunity for disease intervention}

The most informative experiment that will follow our oral potentiation studies in Chapter 5 arises from the CD4-depletion work. We observed that while CD4+ T cells were not required for disease maintenance, the underlaying inflammatory process was diminished following their depletion. Clinically, anti-IgE therapies are effective at desensitizing, but not necessarily tolerizing allergic individuals (permitting long-term disease resolution). However, the $\mathrm{CD} 4+\mathrm{T}$ cell response wanes in anti- $\mathrm{IgE}$ treated individuals[83]. Concomitant CD4-depletion along with administration of anti-IgE in mice (using anti-IgE hybridomas like EM-95[37]), could potentially ablate, and prevent the re-establishment of disease. This may uncover the basic processes that underlie long-term disease susceptibility and eventually lead to a combined therapy that targets allergen-reactive CD4+ T cells and IgE-producing B cells.

It would be ill-advised to use a pan-CD4-depleting antibody (like GK1.5) to treat allergic diseases, because subsets of CD4+ T cells contribute to the adaptive immune response against essentially every pathogen that causes human disease. Therefore, establishing novel ways in which allergen-reacted CD4+ T cells can be targeted will be a second required step that will precede the arrival of potential therapies in the clinic. 


\section{Addressing the "localness" of local responses}

One key aspect that remains to be addressed is the extent to which oral potentiation requires local exposure. Allergen is detected in the intestines of naive allergen-fed mice, but oral challenge of sensitized mice increases intestinal permeability $[37,57]$ and results in 1000-fold higher (to $\mu \mathrm{g} / \mathrm{mL}$ concentrations) levels of allergen in the blood $[68$, 149]. Furthermore, in sensitized mice, systemic anaphylactic responses, in some cases, are caused by oral allergen administration[67], and response severity correlates with the administered allergen dose, and the amount that is absorbed[67, 68]. While the intestinal response requires multiple gavages to become manifest, the dissemination of allergen that follows intestinal allergen exposure argues against complete segregation of each tissue in the common mucosal immune system. In line with the concept of systemic potentiation, oral allergen challenge also increases lung mast cell numbers and exaccerbates the severity of the response evoked by distinct sensitizing allergens in both the lung and the intestine[67, 149, 321]. Clarifying whether the exaccerbation of the lung response requires allergen to reach the lung, or is a general feature of the Th2 response would improve our understanding of the post-sensitization CD4-dependent allergic process. Stabilizing mast cells prior to oral allergen exposure prevents the leaky gut phenotype in iIFABP-IL-9 mice. This could be used to distinguish between systemic potentiation via the oral route and lung potentiation induced by systemic allergen dissemination. This will be an important direction for follow up work to our studies examining oral potentiation of the intestine.

\section{Reflecting allergenicity as a function of Th2 cell development}

In our aim to clarify immunological differences between cow and goat milk, we were unable to identify quantitative differences in the Th2 response to specific proteins. However, closer analysis of the whole casein fraction may identify important aspects of milk allergenicity that our assays have not examined. Specifically, we did not analyse whole caseins in the oral challenge model; ruminant caseins demonstrate significant species-to-species cross-reactivity in humans[34] and this may be an important attribute of their ability to cause allergies. Simply comparing mMCP-1 production 
in the oral challenge model using cow and goat casein fractions, or whole milks in this model, could indicate the ability of milk from each source to promote intestinal allergy. Additionally, in our suppression assays, we examined the ability of milks to suppress the allergic response to an environmental, rather than a food allergen. Future studies would also benefit from analysis of the Th2 response to food allergens in the presence- or absence of milk protein.

\section{The cause of sensitization}

The pathways that initiate sensitization after birth are unclear. Clarifying which aspects of the allergic process are modelled in the two Th2 immune response models examined in this thesis will help us understand how a protein becomes an allergen. In the G4 reporter pTh2 assay, two components should be examined in the near future. First, the influence of adjuvant on the Th2 response should be explored. While we examined the intrinsic allergenicity of homologous proteins in milks, the complex composition of milks may require multiple components to initiate the allergic process. Addition of an adjuvant may amplify Th2 responses to some proteins to a great extent than others. Similarly, cooking processes can modify the structure of isolated proteins and of whole foods[139, 160, 161, 475]; determining whether specific modifications to weak allergens can influence their allergenicity may clarify the processes that contribute to allergic sensitization in future studies. The third aspect implicated in allergic sensitization is the exposure site, and this is not necessarily reflected in the pTh2 assay. Examining allergenicity of primary allergen exposure in the lung, which models inhaled allergen exposure, has been shown to induce Th2-type responses in mice[136]. Using G4 reporter mice it may be possible to quantitate Th2 induction following the first intranasal allergen exposure, and in this reveal additional aspects of the Th2 response to allergens. While this may be below the sensitivity of the initial Th2 response, sufficient Th2 cells may develop, and thus help clarify how allergic Th2 immunity is initiated. 


\subsubsection{Evaluating the success of this project}

In this thesis, "Development of Th2 immune response models to evaluate allergenicity of milk proteins", I aimed to develop assays that could be used to examine the comparative allergenicity of cow and goat milks. While I was unable to identify specific anti-allergic goat milk components, or those with intrinsically lower allergenicity than their homologous cow milk components, I was able to use the PTh2 assay and the oral allergen-induced intestinal anaphylaxis model to test the allergenicity of multiple milk proteins. The findings in chapters three, four and five provide good grounding for the investigations undertaken in these assays. In retrospect, generating empirical data for the mMCP-1 release studies could be informative in determination of the size of the experiments required to observe a predictably 'physiologically relevant' difference in 'allergenicity' in the oral challenge model. Thus, while I was unable to identify immunologic features that result in the reduced allergenicity of goat milk, the studies in this thesis provide clear evidence of how the PTh2 assay and oral challenge model can be used in synchrony as a framework in which multiple features of protein allergenicity can be evaluated.

\subsubsection{Final conclusions}

In this dissertation, I have used literature and experimental evidence to argue for a multi-phase inflammatory process that leads to allergic response susceptibility. The initial allergen exposure initiates IL-4-independent Th2 cell development. These cells are then expanded in the presence of IL-4, facillitate IgE production and are additionally involved in an oral potentiation phase that permits allergic response triggering in the intestine. These processes can be initiated by many individual proteins, but these vary by orders of magnitude, depending on the specific allergen involved. In this manner, allergic disease can be considered an unbalanced sum of factors: Disease is influenced by the inflammatory environment, properties of the allergen and the host that ultimately result in allergic response susceptibility.

The findings used to create this thesis can be summarised in four statements. First, primary allergen immunisation induces IL-4-independent Th2 cell development which 
is followed by an IL-4-dependent phase. Second, IL-4 heterozygous mice are haploinsufficient for IL-4 in allergic disease contexts. Third, CD4+ T cells are critical mediators of IL-3-dependent oral allergen-induced intestinal mastocytosis. Lastly, the low allergenicity of goat milk compared to cow milk relates to its absolute composition, or changes occurring during physiological processing, rather than differences in the intrinsic allergenicity of its dominant protein components.

Despite some significant advances made in recent times, fuller understanding is required before allergies will become manageable maladies that are considered afflictions of the past. The most critical question that remains to be addressed is what permits persistent, consistently deleterious immune responses to normal, everyday proteins and foods. The aetiology of allergic disease is, and should remain at the forefront of scientific research until we have the answer. 


\section{References}

[1] van Panhuys N, Tang SC, Prout M, Camberis M, Scarlett D, Roberts J, Hu-Li J, Paul WE, and Le Gros G. In vivo studies fail to reveal a role for IL-4 or STAT6 signaling in Th2 lymphocyte differentiation. Proceedings of the National Academy of Sciences of the United States of America, 105(34):1242312428, 2008.

[2] Cohen SGS and Zelaya-Quesada MM. Portier, Richet, and the discovery of anaphylaxis: a centennial., vol. 110. Journal of Allergy and Clinical Immunology, National Institute of Allergy and Infectious Diseases, National Institutes of Health, Bethesda, MD, USA., 2002.

[3] Portier M and Richet C. De I'Action Anaphylactique de Certains Venins (Actinotoxine). Comptes rendus Hebdomadaires des seances et Memoires de la Societe de biologie, 54:170-172, 1902.

[4] Sampson HA. Food anaphylaxis. British medical bulletin, 56(4):925-935, 2000.

[5] Sampson HA, Sicherer SH, and Birnbaum AH. AGA technical review on the evaluation of food allergy in gastrointestinal disorders. Gastroenterology, 120(4):1026-1040, 2001.

[6] Brown AFT. Current management of anaphylaxis. Emergencias, 21(3):213223, 2009.

[7] Pumphrey RSH and Gowland MH. Further fatal allergic reactions to food in the United Kingdom, 1999-2006. Journal of Allergy and Clinical Immunology, 119(4):1018-1019, 2007.

[8] Pumphrey RS. Lessons for management of anaphylaxis from a study of fatal reactions. Clinical and experimental allergy : journal of the British Society for Allergy and Clinical Immunology, 30(8):1144-1150, 2000.

[9] Sicherer SH and Sampson HA. Food allergy. The Journal of allergy and clinical immunology, 125(S2):S116-S125, 2010.

[10] Burks AW, Tang M, Sicherer S, Muraro A, Eigenmann PA, Ebisawa M, Fiocchi A, Chiang W, Beyer K, Wood R, Hourihane J, Jones SM, Lack G, and Sampson HA. ICON: Food allergy. The Journal of allergy and clinical immunology, 129(4):906-920, 2012.

[11] NIAID-Sponsored Expert Panel, Boyce JA, Assa'ad A, Burks AW, 
Jones SM, Sampson HA, Wood RA, Plaut M, Cooper SF, Fenton MJ, Arshad SH, Bahna SL, Beck LA, Byrd-Bredbenner C, Camargo CA, Eichenfield L, Furuta GT, Hanifin JM, Jones C, Kraft M, Levy BD, Lieberman P, Luccioli S, McCall KM, Schneider LC, Simon RA, Simons FER, Teach SJ, Yawn BP, and Schwaninger JM. Guidelines for the diagnosis and management of food allergy in the United States: report of the NIAID-sponsored expert panel. In The Journal of allergy and clinical immunology, pp. S1-58. Division of Rheumatology, Immunology and Allergy, Brigham and Women's Hospital, Department of Medicine, Harvard Medical School, Boston, MA, USA., 2010.

[12] Branum AM and Lukacs SL. Food Allergy Among Children in the United States. PEDIATRICS, 124(6):1549-1555, 2009.

[13] Lisker R, Aguilar L, and Zavala C. Intestinal lactase deficiency and milk drinking capacity in the adult. American Journal of Clinical Nutrition, 31(9):1499-1503, 1978.

[14] Kyaw MH and Mayberry JF. Fructose malabsorption: true condition or a variance from normality. Journal of clinical gastroenterology, 45(1):16-21, 2011.

[15] Gijsbers C, Kneepkens C, and Büller HA. Lactose and fructose malabsorption in children with recurrent abdominal pain: results of double-blinded testing. Acta Paediatrica, 101(9):e411-e415, 2012.

[16] Thompson WG, Longstreth GF, Drossman DA, Heaton KW, Irvine EJ, and Müller-Lissner SA. Functional bowel disorders and functional abdominal pain. Gut, 45 Suppl 2:II43-7, 1999.

[17] Sandler RS and Drossman DA. Bowel habits in young adults not seeking health care. Digestive diseases and sciences, 32(8):841-845, 1987.

[18] Tack J. Functional Diarrhea. Gastroenterology Clinics of NA, 41(3):629-637, 2012.

[19] Lack G. Update on risk factors for food allergy. The Journal of allergy and clinical immunology, 129(5):1187-1197, 2012.

[20] López-Expósito I, Song Y, JArvinen KM, Srivastava K, and Li XM. Maternal peanut exposure during pregnancy and lactation reduces peanut allergy risk in offspring. Journal of Allergy and Clinical Immunology, 124(5):10391046, 2009.

[21] Martorell A, Plaza AM, Boné J, Nevot S, García Ara MC, Echeverria L, Alonso E, Garde J, Vila B, Alvaro M, Tauler E, Hernando V, and 
Fernández M. Cow's milk protein allergy. A multi-centre study: clinical and epidemiological aspects. Allergologia et Immunopathologia, 34(2):46-53, 2006.

[22] Zuberbier T. Nahrungsmittelallergien: Epidemiologie in Berlin. Allergologie, 31(7), 2008.

[23] Ishizaka K, Ishizaka T, and Hornbrook MM. Physico-chemical properties of human reaginic antibody. IV. Presence of a unique immunoglobulin as a carrier of reaginic activity. Journal of Immunology, 97(1):75-85, 1966.

[24] Ishizaka K, Ishizaka T, and Hornbrook MM. Physicochemical properties of reaginic antibody. V. Correlation of reaginic activity with gamma-E-globulin antibody. Journal of Immunology, 97(6):840-853, 1966.

[25] Ishizaka K and Ishizaka T. Identification of gamma-E-antibodies as a carrier of reaginic activity. Journal of Immunology, 99(6):1187-1198, 1967.

[26] Lee TD, Sterk A, Ishizaka T, Bienenstock J, and Befus AD. Number and affinity of receptors for IgE on enriched populations of isolated rat intestinal mast cells. Immunology, 55(2):363-366, 1985.

[27] Ishizaka K, Tomioka H, and Ishizaka T. Mechanisms of passive sensitization. I. Presence of IgE and IgG molecules on human leukocytes. Journal of Immunology, 105(6):1459-1467, 1970.

[28] Min B. Basophils: what they 'can do' versus what they 'actually do'. Nature Immunology, 9(12):1333-1339, 2008.

[29] Hakimi J, Seals C, Kondas JA, Pettine L, Danho W, and Kochan J. The alpha subunit of the human IgE receptor (FcERI) is sufficient for high affinity IgE binding. The Journal of biological chemistry, 265(36):22079-22081, 1990 .

[30] Kawakami T and Galli SJ. Regulation of mast-cell and basophil function and survival by IgE. Nature Reviews Immunology, 2(10):773-786, 2002.

[31] Sampson HA. Utility of food-specific IgE concentrations in predicting symptomatic food allergy. Journal of Allergy and Clinical Immunology, 107(5):891896, 2001.

[32] Savage JH, Kaeding AJ, Matsui EC, and Wood RA. The natural history of soy allergy. The Journal of allergy and clinical immunology, 125(3):683-686, 2010 .

[33] Savage JH, Matsui EC, Skripak JM, and Wood RA. The natural history 
of egg allergy. Journal of Allergy and Clinical Immunology, 120(6):1413-1417, 2007.

[34] Sicherer SH and Sampson HA. Cow's milk protein-specific IgE concentrations in two age groups of milk-allergic children and in children achieving clinical tolerance. Clinical and experimental allergy : journal of the British Society for Allergy and Clinical Immunology, 29(4):507-512, 1999.

[35] Skripak JM, Matsui EC, Mudd K, and Wood RA. The natural history of IgE-mediated cow's milk allergy. Journal of Allergy and Clinical Immunology, 120(5):1172-1177, 2007.

[36] Ahrens R, Osterfeld H, Wu D, Chen CY, Arumugam M, Groschwitz K, Strait R, Wang YH, Finkelman FD, and Hogan SP. Intestinal Mast Cell Levels Control Severity of Oral Antigen-Induced Anaphylaxis in Mice. AJPA, 180(4):1535-1546, 2012.

[37] Brandt EB, Strait RT, Hershko D, Wang Q, Muntel EE, Scribner TA, Zimmermann N, Finkelman FD, and Rothenberg ME. Mast cells are required for experimental oral allergen-induced diarrhea. The Journal of clinical investigation, 112(11):1666-1677, 2003.

[38] Knight AK, Blazquez AB, Zhang S, Mayer L, Sampson HA, and Berin MC. CD4 T cells activated in the mesenteric lymph node mediate gastrointestinal food allergy in mice. AJP: Gastrointestinal and Liver Physiology, 293(6):G1234-G1243, 2007.

[39] Arumugam M, Ahrens R, Osterfeld H, Kottyan LC, Shang X, Maclennan JA, Zimmermann N, Zheng Y, Finkelman FD, and Hogan SP. Increased susceptibility of $129 \mathrm{SvEvBrd}$ mice to IgE-Mast cell mediated anaphylaxis. BMC Immunology, 12(1):14, 2011.

[40] Tsujimura Y, Obata K, Mukai K, Shindou H, Yoshida M, Nishikado H, Kawano Y, Minegishi Y, Shimizu T, and Karasuyama H. Basophils Play a Pivotal Role in Immunoglobulin-G-Mediated but Not ImmunoglobulinE-Mediated Systemic Anaphylaxis. Immunity, 28(4):581-589, 2008.

[41] Strait RT, Morris SC, Yang M, Qu XW, and Finkelman FD. Pathways of anaphylaxis in the mouse. Journal of Allergy and Clinical Immunology, 109(4):658-668, 2002.

[42] Song DJ, Cho JY, Miller M, Strangman W, Zhang M, Varki A, and Broide DH. Anti-Siglec-F antibody inhibits oral egg allergen induced intestinal eosinophilic inflammation in a mouse model. Clinical Immunology, 131(1):157169, 2009. 
[43] Feyerabend TB, Weiser A, Tietz A, Stassen M, Harris N, Kopf M, Radermacher P, Möller P, Benoist C, Mathis D, Fehling HJ, and Rodewald HR. Cre-Mediated Cell Ablation Contests Mast Cell Contribution in Models of Antibody- and T Cell-Mediated Autoimmunity. Immunity, pp. $1-13,2011$.

[44] Oliver MR, Tan DT, and Scott RB. Colonic motor response to IgEmediated mast cell degranulation in the Hooded-Lister rat. Neurogastroenterology and motility : the official journal of the European Gastrointestinal Motility Society, 8(2):121-130, 1996.

[45] Kitaura J, Song J, Tsai M, Asai K, Maeda-Yamamoto M, Mocsai A, Kawakami Y, Liu FT, Lowell CA, Barisas BG, Galli SJ, and Kawakami T. Evidence that $\operatorname{IgE}$ molecules mediate a spectrum of effects on mast cell survival and activation via aggregation of the FcepsilonRI. Proceedings of the National Academy of Sciences of the United States of America, 100(22):1291112916, 2003.

[46] Jancar S, Sirois MG, Carrier J, Braquet P, and Sirois P. PAF induces rat plasma extravasation and releases eicosanoids during anaphylaxis. Inflammation, 15(5):347-354, 1991.

[47] Finkelman FD. Anaphylaxis: Lessons from mouse models. Journal of Allergy and Clinical Immunology, 120(3):506-515, 2007.

[48] Strait RTR, Morris SCS, Smiley KK, Urban JFJ, and Finkelman FDF. IL-4 exacerbates anaphylaxis. Journal of Immunology, 170(7):3835-3842, 2003.

[49] Arias K, Baig M, Colangelo M, Chu D, Walker T, Goncharova S, Coyle A, Vadas P, Waserman S, and Jordana M. Concurrent blockade of platelet-activating factor and histamine prevents life-threatening peanutinduced anaphylactic reactions. Journal of Allergy and Clinical Immunology, 124(2):307-314.e2, 2009.

[50] Finkelman FD, Shea-Donohue T, Morris SC, Gildea L, Strait R, Madden KB, Schopf L, and Urban JF. Interleukin-4- and interleukin-13mediated host protection against intestinal nematode parasites. Immunological Reviews, 201(1):139-155, 2004.

[51] Telford GL and Sarna SK. The migrating myoelectric complex of the small intestine. Chaos (Woodbury, N.Y.), 1(3):299-302, 1991.

[52] Scott RB and Tan DT. Mediation of altered motility in food protein induced 
intestinal anaphylaxis in Hooded-Lister rat. Canadian journal of physiology and pharmacology, 74(3):320-330, 1996.

[53] Maric M, Gall DG, and Scott RB. The effect of food protein-induced intestinal anaphylaxis on rate of transit. Canadian journal of physiology and pharmacology, 67(11):1437-1441, 1989.

[54] Oliver MR, Tan DT, and Scott RB. Intestinal anaphylaxis: mediation of the response of colonic longitudinal muscle in rat. The American journal of physiology, 268(5 Pt 1):G764-71, 1995.

[55] Mourad FH, O'Donnell LJ, Ogutu E, Dias JA, and Farthing MJ. Role of 5-hydroxytryptamine in intestinal water and electrolyte movement during gut anaphylaxis. Gut, 36(4):553-557, 1995.

[56] Hanane N, Hanane K, Omar K, and Djamel S. A model of intestinal anaphylaxis in whey sensitized Balb/c mice. American Journal of Immunology, $5(2): 56-60,2009$.

[57] Forbes EE, Groschwitz K, Abonia JP, Brandt EB, Cohen E, Blanchard C, Ahrens R, Seidu L, McKenzie A, Strait R, Finkelman FD, Foster PS, Matthaei KI, Rothenberg ME, and Hogan SP. IL-9- and mast cell-mediated intestinal permeability predisposes to oral antigen hypersensitivity. Journal of Experimental Medicine, 205(4):897-913, 2008.

[58] Kweon MN, Yamamoto M, Kajiki M, Takahashi I, and Kiyono H. Systemically derived large intestinal CD4(+) Th2 cells play a central role in STAT6mediated allergic diarrhea. Journal of Clinical Investigation, 106(2):199-206, 2000 .

[59] Scott RB, Diamant SC, and Gall DG. Motility effects of intestinal anaphylaxis in the rat. The American journal of physiology, 255(4 Pt 1):G505-11, 1988.

[60] Sampson HA. Food allergy. Part 1: immunopathogenesis and clinical disorders. The Journal of allergy and clinical immunology, 103(5 Pt 1):717-728, 1999.

[61] Andrews PLR and Horn CC. Signals for nausea and emesis: Implications for models of upper gastrointestinal diseases. Autonomic Neuroscience, 125(12):100-115, 2006.

[62] Weil AJ and Reddin Jr L. Dermal supersensitivity, heat-labile, and heatstabile antibody against ragweed in cattle. Journal of Immunology, 47:345-352, 1943. 
[63] Yamaki K and Yoshino S. Tyrosine kinase inhibitor sunitinib relieves systemic and oral antigen-induced anaphylaxes in mice. Allergy, 67(1):114-122, 2011.

[64] Scott RB, Gall DG, and Maric M. Mediation of food protein-induced jejunal smooth muscle contraction in sensitized rats. The American journal of physiology, 259(1 Pt 1):G6-14, 1990.

[65] Scott RB and Maric M. Mediation of anaphylaxis-induced jejunal circular smooth muscle contraction in rats. Digestive diseases and sciences, 38(3):396402, 1993.

[66] Zhao A, McDermott J, Urban JF, Gause W, Madden KB, Yeung KA, Morris SC, Finkelman FD, and Shea-Donohue T. Dependence of IL-4, IL13, and nematode-induced alterations in murine small intestinal smooth muscle contractility on Stat6 and enteric nerves. Journal of Immunology, 171(2):948954, 2003.

[67] Kucuk ZY, Strait R, Khodoun MV, Mahler A, Hogan S, and Finkelman FD. Induction and suppression of allergic diarrhea and systemic anaphylaxis in a murine model of food allergy. Journal of Allergy and Clinical Immunology, pp. 1-6, 2012.

[68] Strait RT, Mahler A, Hogan S, Khodoun M, Shibuya A, and Finkelman FD. Ingested allergens must be absorbed systemically to induce systemic anaphylaxis. Journal of Allergy and Clinical Immunology, pp. 1-9, 2011.

[69] Pullerits T, Praks L, Ristioja V, and Lötvall J. Comparison of a nasal glucocorticoid, antileukotriene, and a combination of antileukotriene and antihistamine in the treatment of seasonal allergic rhinitis. Journal of Allergy and Clinical Immunology, 109(6):949-955, 2002.

[70] Mallet AI, Norris P, Rendell NB, Wong E, and Greaves MW. The effect of disodium cromoglycate and ketotifen on the excretion of histamine and $\mathrm{N}$ tau-methylimidazole acetic acid in urine of patients with mastocytosis. British journal of clinical pharmacology, 27(1):88-91, 1989.

[71] Shichijo M, Inagaki N, Nakai N, Kimata M, Nakahata T, Serizawa I, Iikura Y, Saito H, and Nagai H. The effects of anti-asthma drugs on mediator release from cultured human mast cells. Clinical and experimental allergy : journal of the British Society for Allergy and Clinical Immunology, 28(10):1228-1236, 1998.

[72] Novembre E, Galli E, Landi F, Caffarelli C, Pifferi M, De Marco E, Burastero SE, Calori G, Benetti L, and Bonazza P. Coseasonal sublin- 
gual immunotherapy reduces the development of asthma in children with allergic rhinoconjunctivitis. Journal of Allergy and Clinical Immunology, 114(4):851857, 2004.

[73] Nucera E, Schiavino D, Buonomo A, Pollastrini E, Altomonte G, Pecora V, Decinti M, Lombardo C, and Patriarca G. Sublingual-oral rush desensitization to mixed cow and sheep milk: a case report. Journal of investigational allergology $\mathscr{E}$ clinical immunology : official organ of the International Association of Asthmology (INTERASMA) and Sociedad Latinoamericana de Alergia e Inmunología, 18(3):219-222, 2008.

[74] Durham SR, Emminger W, Kapp A, Colombo G, de Monchy JGR, Rak S, Scadding GK, Andersen JS, Riis B, and Dahl R. Long-term clinical efficacy in grass pollen-induced rhinoconjunctivitis after treatment with SQ-standardized grass allergy immunotherapy tablet. The Journal of allergy and clinical immunology, 125(1):131-138.e7, 2010.

[75] Walker S, Pajno G, Lima M, Wilson D, and Durham S. Grass pollen immunotherapy for seasonal rhinitis and asthma: A randomized, controlled trial. Journal of Allergy and Clinical Immunology, 107(1):87-93, 2001.

[76] Bousquet J, Lockey R, Malling HJ, Alvarez-Cuesta E, Canonica GW, Chapman MD, Creticos PJ, Dayer JM, Durham SR, Demoly P, Goldstein RJ, Ishikawa T, Ito K, Kraft D, Lambert PH, Løwenstein H, Müller U, Norman PS, Reisman RE, Valenta R, Valovirta E, and Yssel H. Allergen immunotherapy: therapeutic vaccines for allergic diseases. World Health Organization. American academy of Allergy, Asthma and Immunology., 1998.

[77] Müller U, Akdis CA, Fricker M, Akdis M, Blesken T, Bettens F, and Blaser K. Successful immunotherapy with T-cell epitope peptides of bee venom phospholipase A2 induces specific T-cell anergy in patients allergic to bee venom. The Journal of allergy and clinical immunology, 101(6 Pt 1):747-754, 1998.

[78] Busse W, Corren J, Lanier BQ, McAlary M, Fowler-Taylor A, Cioppa GD, van As A, and Gupta N. Omalizumab, anti-IgE recombinant humanized monoclonal antibody, for the treatment of severe allergic asthma. Journal of Allergy and Clinical Immunology, 108(2):184-190, 2001.

[79] Holgate S, Casale T, Wenzel S, Bousquet J, Deniz Y, and Reisner C. The anti-inflammatory effects of omalizumab confirm the central role of $\mathrm{IgE}$ in allergic inflammation. Journal of Allergy and Clinical Immunology, 115(3):459$465,2005$. 
[80] Fahy JV, Fleming HE, Wong HH, Liu JT, Su JQ, Reimann J, Fick RB, and Boushey HA. The effect of an anti-IgE monoclonal antibody on the early- and late-phase responses to allergen inhalation in asthmatic subjects. American journal of respiratory and critical care medicine, 155(6):1828-1834, 1997.

[81] Bateman ED, Hurd SS, Barnes PJ, Bousquet J, Drazen JM, FitzGerald M, Gibson P, Ohta K, O'Byrne P, Pedersen SE, Pizzichini E, Sullivan SD, Wenzel SE, and Zar HJ. Global strategy for asthma management and prevention: GINA executive summary. European Respiratory Journal, 31(1):143-178, 2008.

[82] Brightbill HD, Jeet S, Lin Z, Yan D, Zhou M, Tan M, Nguyen A, Yeh S, Delarosa D, Leong SR, Wong T, Chen Y, Ultsch M, Luis E, Ramani SR, Jackman J, Gonzalez L, Dennis MS, Chuntharapai A, DeForge L, Meng YG, Xu M, Eigenbrot C, Lee WP, Refino CJ, Balazs M, and Wu LC. Antibodies specific for a segment of human membrane IgE deplete IgE-producing B cells in humanized mice. Journal of Clinical Investigation, 120(6):2218-2229, 2010.

[83] Bedoret D, Singh AK, Shaw V, Hoyte EG, Hamilton R, DeKruyff RH, Schneider LC, Nadeau KC, and Umetsu DT. Changes in antigenspecific T-cell number and function during oral desensitization in cow\&apos;s milk allergy enabled with omalizumab. Mucosal Immunology, 5(3):267-276, 2012 .

[84] Savage JH, Courneya JP, Sterba PM, MacGlashan DW, Saini SS, and Wood RA. Kinetics of mast cell, basophil, and oral food challenge responses in omalizumab-treated adults with peanut allergy. The Journal of allergy and clinical immunology, pp. 1-9, 2012.

[85] Beck LA, Marcotte GV, MacGlashan Jr D, Togias A, and Saini S. Omalizumab-induced reductions in mast cell FcRI expression and function. Journal of Allergy and Clinical Immunology, 114(3):527-530, 2004.

[86] Sampson HA. Food allergy. Part 2: diagnosis and management. The Journal of allergy and clinical immunology, 103(6):981-989, 1999.

[87] Sicherer SH and Teuber S. Current approach to the diagnosis and management of adverse reactions to foods. Journal of Allergy and Clinical Immunology, 114(5):1146-1150, 2004.

[88] Sicherer SH. Food protein-induced enterocolitis syndrome: Case presenta- 
tions and management lessons. Journal of Allergy and Clinical Immunology, 115(1):149-156, 2005.

[89] Shek LPC, Bardina L, Castro R, Sampson HA, and Beyer K. Humoral and cellular responses to cow milk proteins in patients with milk-induced IgEmediated and non-IgE-mediated disorders. Allergy, 60(7):912-919, 2005.

[90] Lin XP, Magnusson J, Ahlstedt S, Dahlman-Höglund A, Hanson LÅ, Magnusson O, Bengtsson U, and Telemo E. Local allergic reaction in food-hypersensitive adults despite a lack of systemic food-specific IgE. Journal of Allergy and Clinical Immunology, 109(5):879-887, 2002.

[91] Goldman AS, Anderson DW, Sellers WA, Saperstein S, Kniker WT, and Halpern SR. Milk Allergy. I. Oral challenge with Milk Proteins in Allergic Children. PEDIATRICS, 32:425-443, 1963.

[92] Maloney JM, Nowak-Wegrzyn A, and Wang J. Children in the inner city of New York have high rates of food allergy and IgE sensitization to common foods. The Journal of allergy and clinical immunology, 128(1):214-215, 2011.

[93] Meyer R, Venter C, Fox AT, and Shah N. Practical dietary management of protein energy malnutrition in young children with cow's milk protein allergy. Pediatric Allergy and Immunology, 23(4):307-314, 2012.

[94] Nowak-Wegrzyn A, Assa'ad AH, Bahna SL, Bock SA, Sicherer SH, Teuber SS, and Adverse Reactions to Food Committee of the American Academy of Allergy AI. Work Group report: Oral food challenge testing. The Journal of allergy and clinical immunology, 123(6):S365-S383, 2009.

[95] Lack G. Epidemiologic risks for food allergy. Journal of Allergy and Clinical Immunology, 121(6):1331-1336, 2008.

[96] Holloway JW, Yang IA, and Holgate ST. Genetics of allergic disease. The Journal of allergy and clinical immunology, 125(S2):S81-S94, 2010.

[97] Spergel J. Atopic dermatitis and the atopic march. Journal of Allergy and Clinical Immunology, 112(6):S118-S127, 2003.

[98] Haller G, Torgerson DG, Ober C, and Thompson EE. Sequencing the IL4 locus in African Americans implicates rare noncoding variants in asthma susceptibility. The Journal of allergy and clinical immunology, 124(6):0-0, 2009.

[99] Wu X, Di Rienzo A, and Ober C. A population genetics study of single nucleotide polymorphisms in the interleukin 4 receptor alpha (IL4RA) gene. Genes and immunity, 2001. 
[100] Hershey GK, Friedrich MF, Esswein LA, Thomas ML, and Chatila TA. The association of atopy with a gain-of-function mutation in the alpha subunit of the interleukin-4 receptor. The New England journal of medicine, 337(24):1720-1725, 1997.

[101] Weidinger S, Illig T, Baurecht H, Irvine AD, Rodriguez E, DiazLacava A, Klopp N, Wagenpfeil S, Zhao Y, Liao H, Lee SP, Palmer CNA, Jenneck C, Maintz L, Hagemann T, Behrendt H, Ring J, Nothen MM, McLean WHI, and Novak N. Loss-of-function variations within the filaggrin gene predispose for atopic dermatitis with allergic sensitizations. The Journal of allergy and clinical immunology, 118(1):214-219, 2006.

[102] Marenholz I, Nickel R, Ruschendorf F, Schulz F, Esparzagordillo J, Kerscher T, Gruber C, Lau S, Worm M, and Keil T. Filaggrin lossof-function mutations predispose to phenotypes involved in the atopic march. Journal of Allergy and Clinical Immunology, 118(4):866-871, 2006.

[103] Kumar R, Tsai HJ, Hong X, Liu X, Wang G, Pearson C, Ortiz K, Fu M, Pongracic JA, Bauchner H, and Wang X. Race, Ancestry, and Development of Food-Allergen Sensitization in Early Childhood. PEDIATRICS, 128(4):821-829, 2011.

[104] Aas K. What makes an allergen an allergen. Allergy, 33(1):3-14, 1978.

[105] Bisgaard H, Li N, Bonnelykke K, Chawes BLK, Skov T, PaludanMüller G, Stokholm J, Smith B, and Krogfelt KA. Reduced diversity of the intestinal microbiota during infancy is associated with increased risk of allergic disease at school age. The Journal of allergy and clinical immunology, 128(3):646-652.e5, 2011.

[106] Rautava S, Kainonen E, Salminen S, and Isolauri E. Maternal probiotic supplementation during pregnancy and breast-feeding reduces the risk of eczema in the infant. The Journal of allergy and clinical immunology, pp. -, 2012.

[107] Wickens K, Black PN, Stanley TV, Mitchell E, Fitzharris P, Tannock GW, Purdie G, and Crane J. A differential effect of 2 probiotics in the prevention of eczema and atopy: A double-blind, randomized, placebo-controlled trial. Journal of Allergy and Clinical Immunology, 122(4):788-794, 2008.

[108] Perkin M and Strachan D. Which aspects of the farming lifestyle explain the inverse association with childhood allergy? Journal of Allergy and Clinical Immunology, 117(6):1374-1381, 2006.

[109] Eggesbø M, Botten G, Stigum H, Nafstad P, and Magnus P. Is delivery 
by cesarean section a risk factor for food allergy? Journal of Allergy and Clinical Immunology, 112(2):420-426, 2003.

[110] Laubereau B. Caesarean section and gastrointestinal symptoms, atopic dermatitis, and sensitisation during the first year of life. Archives of Disease in Childhood, 89(11):993-997, 2004.

[111] Ma B, Forney LJ, and Ravel J. Vaginal microbiome: rethinking health and disease. Annual review of microbiology, 66:371-389, 2012.

[112] Antonopoulos DA, Huse SM, Morrison HG, Schmidt TM, Sogin ML, and Young VB. Reproducible Community Dynamics of the Gastrointestinal Microbiota following Antibiotic Perturbation. Infection and immunity, 77(6):2367-2375, 2009.

[113] Hill DA, Siracusa MC, Abt MC, Kim BS, Kobuley D, Kubo M, Kambayashi T, LaRosa DF, Renner ED, Orange JS, Bushman FD, and Artis D. Commensal bacteria\&ndash;derived signals regulate basophil hematopoiesis and allergic inflammation. Nature Medicine, 18(4):538-546, 2012.

[114] Taylor RR, Sladkevicius E, Panca M, Lack G, and Guest JF. Costeffectiveness of using an extensively hydrolysed formula compared to an amino acid formula as first-line treatment for cow milk allergy in the UK. Pediatric Allergy and Immunology, 23(3):240-249, 2012.

[115] Jones AP, Palmer D, Zhang G, and Prescott SL. Cord Blood 25Hydroxyvitamin D3 and Allergic Disease During Infancy. PEDIATRICS, pp. -, 2012 .

[116] Litonjua AA. Vitamin D deficiency as a risk factor for childhood allergic disease and asthma. Current opinion in allergy and clinical immunology, 12(2):179$185,2012$.

[117] Mullins RJ, Clark S, Wiley V, Eyles D, and Camargo CA. Neonatal vitamin D status and childhood peanut allergy: a pilot study. Annals of Allergy, Asthma and Immunology, 109(5):324-328, 2012.

[118] Rühl R, Hänel A, Garcia AL, Dahten A, Herz U, Schweigert FJ, and Worm M. Role of vitamin A elimination or supplementation diets during postnatal development on the allergic sensitisation in mice. Molecular Nutrition \& Food Research, 51(9):1173-1181, 2007.

[119] Sampson M, Munozfurlong A, and Sicherer S. Risk-taking and coping strategies of adolescents and young adults with food allergy. Journal of Allergy and Clinical Immunology, 117(6):1440-1445, 2006. 
[120] Ngamphaiboon JJ, Chatchatee PP, and Thongkaew TT. Cow's milk allergy in Thai children. Asian Pacific Journal of Allergy 65 Immunology, 26(4):199-204, 2008.

[121] Fox AT, Du Toit G, Lang A, and Lack G. Food allergy as a risk factor for nutritional rickets. Pediatric allergy and immunology : official publication of the European Society of Pediatric Allergy and Immunology, 15(6):566-569, 2004.

[122] Maslowski KM, Vieira AT, Ng A, Kranich J, Sierro F, Yu D, Schilter HC, Rolph MS, Mackay F, Artis D, Xavier RJ, Teixeira MM, and Mackay CR. Regulation of inflammatory responses by gut microbiota and chemoattractant receptor GPR43. Nature, 461(7268):1282-1286, 2009.

[123] Strachan DP. Hay fever, hygiene, and household size. BMJ, 299(6710):1259 1260, 1989.

[124] Rosenstreich DL, Eggleston P, Kattan M, Baker D, Slavin RG, Gergen $\mathrm{P}$, Mitchell $\mathrm{H}$, McNiff-Mortimer $\mathrm{K}$, Lynn $\mathrm{H}$, Ownby $\mathrm{D}$, and Malveaux F. The role of cockroach allergy and exposure to cockroach allergen in causing morbidity among inner-city children with asthma. The New England journal of medicine, 336(19):1356-1363, 1997.

[125] Lack G, Fox D, Northstone K, Golding J, and Avon Longitudinal Study of Parents and Children Study Team. Factors associated with the development of peanut allergy in childhood. The New England journal of medicine, 348(11):977-985, 2003.

[126] Du Toit G, Katz Y, Sasieni P, Mesher D, Maleki SJ, Fisher HR, Fox AT, Turcanu V, Amir T, Zadik-Mnuhin G, Cohen A, Livne I, and Lack G. Early consumption of peanuts in infancy is associated with a low prevalence of peanut allergy. The Journal of allergy and clinical immunology, 122(5):984-991, 2008.

[127] Fox AT, Sasieni P, Du Toit G, Syed H, and Lack G. Household peanut consumption as a risk factor for the development of peanut allergy. The Journal of allergy and clinical immunology, 123(2):417-423, 2009.

[128] Peters JL, Suglia SF, Platts-Mills TAE, Hosen J, Gold DR, and Wright RJ. Relationships among prenatal aeroallergen exposure and maternal and cord blood IgE: project ACCESS. The Journal of allergy and clinical immunology, 123(5):1041-1046, 2009.

[129] Sicherer SH and Burks AW. Maternal and infant diets for prevention of allergic diseases: Understanding menu changes in 2008. Journal of Allergy and Clinical Immunology, 122(1):29-33, 2008. 
[130] Pabst O and Mowat AM. Oral tolerance to food protein. Mucosal Immunology, 5(3):232-239, 2012.

[131] Hanson LA, Lundin S, Karlsson M, Dahlman-Hoglund A, Dahlgren U, and Telemo E. Regulation of the immune response to oral administration of antigens in food allergic experimental animals. In Nutrition Research, pp. 1343-1350. Univ Gothenburg, Dept Clin Immunol, Goteborg, Sweden, 1998.

[132] Blázquez AB, Mayer L, and Berin MC. Thymic stromal lymphopoietin is required for gastrointestinal allergy but not oral tolerance. Gastroenterology, 139(4):1301-1309, 2010.

[133] Verhasselt V, Milcent V, Cazareth J, Kanda A, Fleury S, Dombrowicz D, Glaichenhaus N, and Julia V. Breast milk-mediated transfer of an antigen induces tolerance and protection from allergic asthma. Nature Medicine, 14(2):170-175, 2008.

[134] Worbs T. Oral tolerance originates in the intestinal immune system and relies on antigen carriage by dendritic cells. Journal of Experimental Medicine, 203(3):519-527, 2006.

[135] Leonard SA, Martos G, Wang W, Nowak-Wegrzyn A, and Berin MC. Oral immunotherapy induces local protective mechanisms in the gastrointestinal mucosa. The Journal of allergy and clinical immunology, pp. 1-10, 2012.

[136] Nelde A, Teufel M, Hahn C, Duschl A, Sebald W, Bröcker EB, and Grunewald SM. The impact of the route and frequency of antigen exposure on the IgE response in allergy. International Archives of Allergy and Immunology, 124(4):461-469, 2001.

[137] Husby S, Mestecky J, Moldoveanu Z, Holland S, and Elson CO. Oral tolerance in humans. T cell but not B cell tolerance after antigen feeding. Journal of Immunology, 152(9):4663-4670, 1994.

[138] Spazierer D, Skvara H, Dawid M, Fallahi N, Gruber K, Rose K, Lloyd P, Heuerding S, Stingl G, and Jung T. T helper 2 biased de novoimmune response to Keyhole Limpet Hemocyanin in humans. Clinical $\&$ Experimental Allergy, 39(7):999-1008, 2009.

[139] Roth-Walter F, Berin MC, Arnaboldi P, Escalante CR, Dahan S, Rauch J, Jensen-Jarolim E, and Mayer L. Pasteurization of milk proteins promotes allergic sensitization by enhancing uptake through Peyer's patches. Allergy, 63(7):882-890, 2008.

[140] Snider DP, Marshall JS, Perdue MH, and Liang H. Production of 
$\operatorname{IgE}$ antibody and allergic sensitization of intestinal and peripheral tissues after oral immunization with protein Ag and cholera toxin. Journal of Immunology, 153(2):647-657, 1994.

[141] Sanz Ceballos L, Sanz Sampelayo MR, Gil Extremera F, and Rodríguez Osorio M. Evaluation of the allergenicity of goat milk, cow milk, and their lactosera in a guinea pig model. Journal of dairy science, 92(3):837$846,2009$.

[142] Mowat AM. Anatomical basis of tolerance and immunity to intestinal antigens. Nature Reviews Immunology, 3(4):331-341, 2003.

[143] Hadis U, Wahl B, Schulz O, Hardtke-Wolenski M, Schippers A, Wagner N, Müller W, Sparwasser T, Förster R, and Pabst O. Intestinal Tolerance Requires Gut Homing and Expansion of FoxP3+ Regulatory T Cells in the Lamina Propria. Immunity, 34(2):237-246, 2011.

[144] Grindebacke H, Stenstad H, Quiding-Jarbrink M, Waldenstrom J, Adlerberth I, Wold AE, and Rudin A. Dynamic Development of Homing Receptor Expression and Memory Cell Differentiation of Infant CD4+CD25high Regulatory T Cells. The Journal of Immunology, 183(7):4360-4370, 2009.

[145] Rescigno M, Urbano M, Valzasina B, Francolini M, Rotta G, Bonasio R, Granucci F, Kraehenbuhl JP, and Ricciardi-Castagnoli P. Dendritic cells express tight junction proteins and penetrate gut epithelial monolayers to sample bacteria. Nature Immunology, 2(4):361-367, 2001.

[146] Frossard CP, Tropia L, Hauser C, and Eigenmann PA. Lymphocytes in Peyer patches regulate clinical tolerance in a murine model of food allergy. Journal of Allergy and Clinical Immunology, 113(5):958-964, 2004.

[147] Tsuji NM, Mizumachi K, and Kurisaki J. Interleukin-10-secreting Peyer's patch cells are responsible for active suppression in low-dose oral tolerance. Immunology, 103(4):458-464, 2001.

[148] Karlsson M, Lundin S, Dahlgren U, Kahu H, Pettersson I, and Telemo E. "Tolerosomes" are produced by intestinal epithelial cells. European journal of immunology, 31(10):2892-2900, 2001.

[149] Li XM, Schofield BH, Huang CK, Kleiner GI, and Sampson HA. A murine model of IgE-mediated cow's milk hypersensitivity. The Journal of allergy and clinical immunology, 103(2 Pt 1):206-214, 1999.

[150] Walzer M. Studies in absorption of undigested proteins in human beings I. A 
simple direct method of studying the absorption of undigested protein. Journal of Immunology, 14(3):143-174, 1927.

[151] Peng HJ, Turner MW, and Strobel S. The generation of a 'tolerogen' after the ingestion of ovalbumin is time-dependent and unrelated to serum levels of immunoreactive antigen. Clinical and experimental immunology, 81(3):510-515, 1990.

[152] Chapman MD, Pomés A, Breiteneder H, and Ferreira F. Nomenclature and structural biology of allergens. Journal of Allergy and Clinical Immunology, 119(2):414-420, 2007.

[153] Shah R and Grammer LC. Chapter 1: An overview of allergens. Allergy and Asthma Proceedings, 33(3):2-5, 2012.

[154] Ferreira F, Hawranek T, Gruber P, Wopfner N, and Mari A. Allergic cross-reactivity: from gene to the clinic. Allergy, 59(3):243-267, 2004.

[155] Radauer C, Bublin M, Wagner S, Mari A, and Breiteneder H. Allergens are distributed into few protein families and possess a restricted number of biochemical functions. Journal of Allergy and Clinical Immunology, 121(4):847852.e7, 2008.

[156] Bernhisel-Broadbent J, Dintzis HM, Dintzis RZ, and Sampson HA. Allergenicity and antigenicity of chicken egg ovomucoid (Gal d III) compared with ovalbumin (Gal d I) in children with egg allergy and in mice. Journal of Allergy and Clinical Immunology, 93(6):1047-1059, 1994.

[157] Trompette A, Divanovic S, Visintin A, Blanchard C, Hegde RS, Madan R, Thorne PS, Wills-Karp M, Gioannini TL, Weiss JP, and Karp CL. Allergenicity resulting from functional mimicry of a Toll-like receptor complex protein. Nature, 457(7229):585-588, 2008.

[158] Boyce JA, Bochner B, Finkelman FD, and Rothenberg ME. Advances in mechanisms of asthma, allergy, and immunology in 2011. Journal of Allergy and Clinical Immunology, 129(2):335-341, 2012.

[159] Beyer K, Morrowa E, Li XM, Bardina L, Bannon GA, Burks AW, and Sampson HA. Effects of cooking methods on peanut allergenicity. Journal of Allergy and Clinical Immunology, 107(6):1077-1081, 2001.

[160] Ilchmann A, Burgdorf S, Scheurer S, Waibler Z, Nagai R, Wellner A, Yamamoto Y, Yamamoto H, Henle T, Kurts C, Kalinke U, Vieths S, and Toda M. Glycation of a food allergen by the Maillard reaction enhances 
its T-cell immunogenicity: Role of macrophage scavenger receptor class A type I and II. The Journal of allergy and clinical immunology, pp. 1-20, 2009.

[161] Maleki SJ, Chung SY, Champagne ET, and Raufman JP. The effects of roasting on the allergenic properties of peanut proteins. The Journal of allergy and clinical immunology, 106(4):763-768, 2000.

[162] Kim JS, Nowak-Wegrzyn A, Sicherer SH, Noone S, Moshier EL, and Sampson HA. Dietary baked milk accelerates the resolution of cows milk allergy in children. The Journal of allergy and clinical immunology, pp. 1-9, 2011.

[163] Aalberse RC and Crameri R. IgE-binding epitopes: a reappraisal. Allergy, 66(10):1261-1274, 2011.

[164] Huby RD, Dearman RJ, and Kimber I. Why are some proteins allergens? Toxicological sciences : an official journal of the Society of Toxicology, 55(2):235-246, 2000.

[165] Traidl-Hoffmann C, Jakob T, and Behrendt H. Determinants of allergenicity. The Journal of allergy and clinical immunology, 123(3):558-566, 2009.

[166] Costa J, Mafra I, Carrapatoso I, and Oliveira MBPP. Almond Allergens: Molecular Characterization, Detection, and Clinical Relevance. Journal of agricultural and food chemistry, 60(6):1337-1349, 2012.

[167] Svedberg T and Sjögren B. The molecular weights of amandin and of excelsin. Journal of the American Chemical Society, 52(1):279-287, 1930.

[168] Sathe SK, Wolf WJ, Roux KH, Teuber SS, Venkatachalam M, and Sze-Tao KWC. Biochemical Characterization of Amandin, the Major Storage Protein in Almond ( Prunus dulcisL.). Journal of agricultural and food chemistry, 50(15):4333-4341, 2002.

[169] Restani P, Ballabio C, Lorenzo C, Tripodi S, and Fiocchi A. Molecular aspects of milk allergens and their role in clinical events. Analytical and Bioanalytical Chemistry, 395(1):47-56, 2009.

[170] Pomés A. Allergen structures and biologic functions: the cutting edge of allergy research. Current allergy and asthma reports, 8(5):425-432, 2008.

[171] Verdino P, Barderas R, Villalba M, Westritschnig K, Valenta R, Rodriguez $\mathbf{R}$, and Keller W. Three-dimensional structure of the cross-reactive pollen allergen Che a 3: visualizing cross-reactivity on the molecular surfaces of weed, grass, and tree pollen allergens. Journal of Immunology, 180(4):2313$2321,2008$. 
[172] Sánchez-Monge R, Lombardero M, García-Sellés FJ, Barber D, and Salcedo G. Lipid-transfer proteins are relevant allergens in fruit allergy. The Journal of allergy and clinical immunology, 103(3 Pt 1):514-519, 1999.

[173] Borges JP, Barre A, Culerrier R, Granier C, Didier A, and Rougé P. Lipid transfer proteins from Rosaceae fruits share consensus epitopes responsible for their IgE-binding cross-reactivity. Biochemical and Biophysical Research Communications, 365(4):685-690, 2008.

[174] Kontopidis G, Holt C, and Sawyer L. Invited review: beta-lactoglobulin: binding properties, structure, and function. Journal of dairy science, 87(4):785$796,2004$.

[175] Kontopidis G, Holt C, and Sawyer L. The Ligand-binding Site of Bovine -Lactoglobulin: Evidence for a Function? Journal of Molecular Biology, 318(4):1043-1055, 2002.

[176] Pérez MD, Diaz de Villegas C, Sanchez L, Aranda P, Ena JM, and Calvo M. Interaction of fatty acids with beta-lactoglobulin and albumin from ruminant milk. Journal of biochemistry, 106(6):1094-1097, 1989.

[177] Papiz MZ, Sawyer L, Eliopoulos EE, North AC, Findlay JB, Sivaprasadarao R, Jones TA, Newcomer ME, and Kraulis PJ. The structure of beta-lactoglobulin and its similarity to plasma retinol-binding protein. Nature, 324(6095):383-385, 1986.

[178] Astwood JD, Leach JN, and Fuchs RL. Stability of food allergens to digestion in vitro. Nature biotechnology, 14(10):1269-1273, 1996.

[179] Menard, Cerf-Bensussan N, and Heyman M. Multiple facets of intestinal permeability and epithelial handling of dietary antigens. Mucosal Immunology, $3(3): 247-259,2010$.

[180] Smole U, Bublin M, Radauer C, Ebner C, and Breiteneder H. Mal d 2, the Thaumatin-Like Allergen from Apple, Is Highly Resistant to Gastrointestinal Digestion and Thermal Processing. International Archives of Allergy and Immunology, 147(4):289-298, 2008.

[181] del Val G, Yee BC, Lozano RM, Buchanan BB, Ermel RW, Lee YM, and Frick OL. Thioredoxin treatment increases digestibility and lowers allergenicity of milk. The Journal of allergy and clinical immunology, 103(4):690697, 1999.

[182] Bernard H, Créminon C, Yvon M, and Wal JM. Specificity of the human 
IgE response to the different purified caseins in allergy to cow's milk proteins. International Archives of Allergy and Immunology, 115(3):235-244, 1998.

[183] Docena GH, Fernandez R, Chirdo FG, and Fossati CA. Identification of casein as the major allergenic and antigenic protein of cow's milk. Allergy, 51(6):412-416, 1996.

[184] Picariello G, Ferranti P, Fierro O, Mamone G, Caira S, Di Luccia A, Monica S, and Addeo F. Peptides surviving the simulated gastrointestinal digestion of milk proteins: Biological and toxicological implications. Journal of Chromatography B, 878(3-4):295-308, 2010.

[185] Almaas H, Cases AL, Devold TG, Holm H, Langsrud T, Aabakken L, Aadnoey T, and Vegarud GE. In vitro digestion of bovine and caprine milk by human gastric and duodenal enzymes. International Dairy Journal, 16(9):961-968, 2006.

[186] Sancho AI, Wangorsch A, Jensen BM, Watson A, Alexeev Y, Johnson PE, Mackie AR, Neubauer A, Reese G, Ballmer-Weber B, HoffmannSommergruber K, Skov PS, Vieths S, and Mills ENC. Responsiveness of the major birch allergen Bet v 1 scaffold to the gastric environment: Impact on structure and allergenic activity. Molecular Nutrition \& Food Research, 55(11):1690-1699, 2011.

[187] Fernández-Rivas M, Bolhaar S, González-Mancebo E, Asero R, van Leeuwen A, Bohle B, Ma Y, Ebner C, Rigby N, and Sancho AI. Apple allergy across Europe: How allergen sensitization profiles determine the clinical expression of allergies to plant foods. Journal of Allergy and Clinical Immunology, 118(2):481-488, 2006.

[188] Newell SJ, Chapman S, and Booth IW. Ultrasonic assessment of gastric emptying in the preterm infant. Archives of Disease in Childhood, 69(1 Spec No):32-36, 1993.

[189] Hamosh M, Scanlon JW, Ganot D, Likel M, Scanlon KB, and Hamosh $\mathbf{P}$. Fat digestion in the newborn. Characterization of lipase in gastric aspirates of premature and term infants. Journal of Clinical Investigation, 67(3):838-846, 1981.

[190] Sicherer SH, MuNoz-Furlong A, Godbold JH, and Sampson HA. US prevalence of self-reported peanut, tree nut, and sesame allergy: 11-year followup. The Journal of allergy and clinical immunology, pp. 1-5, 2010.

[191] Sicherer SH. Clinical implications of cross-reactive food allergens. Journal of Allergy and Clinical Immunology, 108(6):881-890, 2001. 
[192] Bellioni-Businco B, Paganelli R, Lucenti P, Giampietro PG, Perborn $\mathbf{H}$, and Businco L. Allergenicity of goat's milk in children with cow's milk allergy. The Journal of allergy and clinical immunology, 103(6):1191-1194, 1999.

[193] Jarvinen KM, Geller L, Bencharitiwong R, and Sampson HA. Presence of functional, autoreactive human milk-specific $\operatorname{IgE}$ in infants with cow's milk allergy. Clinical $\&$ Experimental Allergy, 42(2):238-247, 2011.

[194] Maynard F, Chatel JM, and Wal JM. Immunological IgE Cross-Reactions of Bovine and Human $\mathrm{i}_{i} \mathrm{i} / \mathrm{i} \mathrm{i}_{-}$-Lactalbumins in Cow's Milk Allergic Patients. Food and Agricultural Immunology, 11(2):179-189, 1998.

[195] Cantisani A, Giuffrida MG, Fabris C, Bertino E, Coscia A, Oggero R, Monti G, Stroppiana P, and Conti A. Detection of specific IgE to human milk proteins in sera of atopic infants. FEBS Letters, 412(3):515-517, 1997.

[196] Bernard H, Negroni L, Chatel JM, Clément G, Adel-Patient K, Peltre G, Créminon C, and Wal JM. Molecular basis of IgE cross-reactivity between human beta-casein and bovine beta-casein, a major allergen of milk. Molecular Immunology, 37(3-4):161-167, 2000.

[197] Schulmeister U, Swoboda I, Quirce S, de la Hoz B, Ollert M, Pauli G, Valenta R, and Spitzauer S. Sensitization to human milk. Clinical $\&$ Experimental Allergy, 0(0):071029193902001-???, 2007.

[198] Kulig M, Bergmann R, Klettke U, Wahn V, Tacke U, and Wahn U. Natural course of sensitization to food and inhalant allergens during the first 6 years of life. The Journal of allergy and clinical immunology, 103(6):1173-1179, 1999.

[199] Vieths S, Jankiewicz A, Schöning B, and Aulepp H. Apple allergy: the IgE-binding potency of apple strains is related to the occurrence of the $18-\mathrm{kDa}$ allergen. Allergy, 49(4):262-271, 1994.

[200] Ebner C, Hirschwehr R, Bauer L, Breiteneder H, Valenta R, Ebner H, Kraft D, and Scheiner O. Identification of allergens in fruits and vegetables: IgE cross-reactivities with the important birch pollen allergens Bet $\mathrm{v} 1$ and Bet v 2 (birch profilin). The Journal of allergy and clinical immunology, 95(5 Pt 1):962-969, 1995.

[201] Campo P, Aranda A, Palacin A, Montañez MI, Díaz-Perales A, and Blanca M. Occupational asthma caused by IgE-mediated sensitization to multiple woods. The Journal of allergy and clinical immunology, 129(1):254-256.e2, 2012 . 
[202] Baur X, Degens PO, and Sander I. Baker's asthma: still among the most frequent occupational respiratory disorders. The Journal of allergy and clinical immunology, 102(6 Pt 1):984-997, 1998.

[203] Dahlman-Höglund A, Renström A, Larsson PH, Elsayed S, and Andersson E. Salmon allergen exposure, occupational asthma, and respiratory symptoms among salmon processing workers. American journal of industrial medicine, 55(7):624-630, 2012.

[204] Brehler R, Theissen U, Mohr C, and Luger T. "Latex-fruit syndrome": frequency of cross-reacting IgE antibodies. Allergy, 52(4):404-410, 1997.

[205] Commins SP, Satinover SM, Hosen J, Mozena J, Borish L, Lewis BD, Woodfolk JA, and Platts-Mills TAE. Delayed anaphylaxis, angioedema, or urticaria after consumption of red meat in patients with $\operatorname{IgE}$ antibodies specific for galactose-alpha-1,3-galactose. The Journal of allergy and clinical immunology, 123(2):426-433.e2, 2009.

[206] Commins SP, Kelly LA, Ronmark E, James HR, Pochan SL, Peters EJ, Lundback B, Nganga LW, Cooper PJ, Hoskins JM, Eapen SS, Matos LA, McBride DC, Heymann PW, Woodfolk JA, Perzanowski MS, and Platts-Mills TAE. Galactose- -1,3-Galactose-Specific IgE Is Associated with Anaphylaxis but Not Asthma. American journal of respiratory and critical care medicine, 185(7):723-730, 2012.

[207] Commins SP, James HR, Kelly LA, Pochan SL, Workman LJ, Perzanowski MS, Kocan KM, Fahy JV, Nganga LW, Ronmark E, Cooper PJ, and Platts-Mills TAE. The relevance of tick bites to the production of IgE antibodies to the mammalian oligosaccharide galactose-alpha-1,3galactose. The Journal of allergy and clinical immunology, 127(5):1286-1293.e6, 2011.

[208] Li S, Schmitz KR, Jeffrey PD, Wiltzius JJW, Kussie P, and Ferguson KM. Structural basis for inhibition of the epidermal growth factor receptor by cetuximab. Cancer Cell, 7(4):301-311, 2005.

[209] Commins SP and Platts-Mills TAE. Delayed Anaphylaxis to Red Meat in Patients with IgE Specific for Galactose alpha-1,3-Galactose (alpha-gal). Current allergy and asthma reports, 2012.

[210] Jacquenet S, Moneret-Vautrin DA, and Bihain BE. Mammalian meatinduced anaphylaxis: Clinical relevance of anti-galactose-alpha-1,3-galactose IgE confirmed by means of skin tests to cetuximab. The Journal of allergy and clinical immunology, 124(3):603-605, 2009. 
[211] Farrell HM, Jimenez-Flores R, Bleck GT, Brown EM, Butler JE, Creamer LK, Hicks CL, Hollar CM, Ng-Kwai-Hang KF, and Swaisgood HE. Nomenclature of the proteins of cows' milk-sixth revision. Journal of dairy science, 87(6):1641-1674, 2004.

[212] Hadjipanayiotou M. Composition of ewe, goat and cow milk and of colostrum of ewes and goats. Small Ruminant Research, 18(3):255-262, 1995.

[213] Martinez-Ferez A, Zapata JE, Guadix A, Almecija MC, Gomez M, and Guadix EM. Obtention of goat milk permeates enriched in lactose-derived oligosaccharides. DES, 245(1-3):730-736, 2009.

[214] Alonso LL, Fontecha JJ, Lozada LL, Fraga MJM, and Juárez MM. Fatty acid composition of caprine milk: major, branched-chain, and trans fatty acids. Journal of dairy science, 82(5):878-884, 1999.

[215] Prosser CG, McLaren RD, Frost D, Agnew M, and Lowry DJ. Composition of the non-protein nitrogen fraction of goat whole milk powder and goat milk-based infant and follow-on formulae. International Journal of Food Sciences and Nutrition, 59(2):123-133, 2008.

[216] Zygoura P, Moyssiadi T, Badeka A, Kondyli E, Savvaidis I, and Kontominas MG. Shelf life of whole pasteurized milk in Greece: effect of packaging material. Food Chemistry, 87(1):1-9, 2004.

[217] do Nascimento IR, de Jesus RM, dos Santos WNL, Souza AS, Fragoso WD, and dos Reis PS. Determination of the mineral composition of fresh bovine milk from the milk-producing areas located in the State of Sergipe in Brazil and evaluation employing exploratory analysis. Microchemical Journal, pp. 1-5, 2010.

[218] Vassila E, Badeka A, Kondyli E, Savvaidis I, and Kontominas MG. Chemical and microbiological changes in fluid milk as affected by packaging conditions. International Dairy Journal, 12(9):715-722, 2002.

[219] Erinoso HO, Hoare S, and Weaver LT. Is cow's milk suitable for the dietary supplementation of rural Gambian children? 2. Patterns of cow's milk intake. Annals of tropical paediatrics, 12(4):367-373, 1992.

[220] Ayele Z and Peacock C. Improving access to and consumption of animal source foods in rural households: the experiences of a women-focused goat development program in the highlands of Ethiopia. The Journal of nutrition, 133(11 Suppl 2):3981S-3986S, 2003.

[221] Ah-Leung S, Bernard H, Bidat E, Paty E, Rancé F, Scheinmann P, 
and Wal JM. Allergy to goat and sheep milk without allergy to cow's milk. Allergy, 61(11):1358-1365, 2006.

[222] Walker A. Breast Milk as the Gold Standard for Protective Nutrients. The Journal of Pediatrics, 156(2 Suppl):0-0, 2010.

[223] Lönnerdal B. Nutritional and physiologic significance of human milk proteins. American Journal of Clinical Nutrition, 77(6):1537S-1543S, 2003.

[224] Lönnerdal B. Bioactive Proteins in Human Milk: Mechanisms of Action. The Journal of Pediatrics, 156(2 Suppl):0-0, 2010.

[225] Jenness RR. The composition of human milk. Seminars in Perinatology, 3(3):225-239, 1979.

[226] Lönnerdal B. Biochemistry and physiological function of human milk proteins. American Journal of Clinical Nutrition, 42(6):1299-1317, 1985.

[227] Khan S, Casadio YS, Lai CT, Prime DK, Hepworth AR, Trengove NJ, and Hartmann PE. Investigation of short-term variations in casein and whey proteins in breast milk of term mothers. Journal of pediatric gastroenterology and nutrition, 55(2):136-141, 2012.

[228] El-Agamy EI, Nawar M, Shamsia SM, Awad S, and Haenlein GFW. Are camel milk proteins convenient to the nutrition of cow milk allergic children? Small Ruminant Research, 82(1):1-6, 2009.

[229] Gao X, McMahon RJ, Woo JG, Davidson BS, Morrow AL, and Zhang Q. Temporal Changes in Milk Proteomes Reveal Developing Milk Functions. Journal of Proteome Research, 11(7):3897-3907, 2012.

[230] Dalgleish DG and Corredig M. The Structure of the Casein Micelle of Milk and Its Changes During Processing. Annual Review of Food Science and Technology, 3(1):449-467, 2012.

[231] Moatsou G, Hatzinaki A, Samolada M, and Anifantakis E. Major whey proteins in ovine and caprine acid wheys from indigenous greek breeds. International Dairy Journal, 15(2):123-131, 2005.

[232] Sanz Ceballos L, Sanz Sampelayo MR, Gil Extremera F, and Rodríguez Osorio M. Antigenic stimulation with goat and cow milk by oral and parenteral route in guinea pigs. Food and Agricultural Immunology, 21(1):1-13, 2010.

[233] Moatsou G, Moschopoulou E, Mollé D, Gagnaire V, Kandarakis I, and Léonil J. Comparative study of the protein fraction of goat milk from 
the Indigenous Greek breed and from international breeds. Food Chemistry, 106(2):509-520, 2008.

[234] Lara-Villoslada F, Olivares M, Jiménez J, Boza J, and Xaus J. Goat milk is less immunogenic than cow milk in a murine model of atopy. Journal of pediatric gastroenterology and nutrition, 39(4):354-360, 2004.

[235] Ceballos LS, Morales ER, de la Torre Adarve G, Castro JD, Martínez LP, and Sampelayo MRS. Composition of goat and cow milk produced under similar conditions and analyzed by identical methodology. Journal of Food Composition and Analysis, 22(4):322-329, 2009.

[236] Ballabio C, Chessa S, Rignanese D, Gigliotti C, Pagnacco G, Terracciano L, Fiocchi A, Restani P, and Caroli AM. Goat milk allergenicity as a function of S1-casein genetic polymorphism. Journal of dairy science, 94(2):998-1004, 2011.

[237] Tomotake H, Okuyama R, Katagiri M, Fuzita M, Yamato M, and Ota F. Comparison between Holstein Cow's Milk and Japanese-Saanen Goat's Milk in Fatty Acid Composition, Lipid Digestibility and Protein Profile. Bioscience, Biotechnology, and Biochemistry, 70(11):2771-2774, 2006.

[238] McLaughlan P, Anderson KJ, Widdowson EM, and Coombs RR. Effect of heat on the anaphylactic-sensitising capacity of cows' milk, gots' milk, and various infant formulae fed to guinea-pigs. Archives of Disease in Childhood, 56(3):165-171, 1981.

[239] Kondyli E, Katsiari MC, and Voutsinas LP. Variations of vitamin and mineral contents in raw goat milk of the indigenous Greek breed during lactation. Food Chemistry, 100(1):226-230, 2007.

[240] Wirmer J, Berk H, Ugolini R, Redfield C, and Schwalbe H. Characterization of the unfolded state of bovine -lactalbumin and comparison with unfolded states of homologous proteins. Protein science : a publication of the Protein Society, 15(6):1397-1407, 2006.

[241] Ginger MRM and Grigor MRM. Comparative aspects of milk caseins. Comparative Biochemistry and Physiology Part B: Biochemistry and Molecular Biology, 124(2):133-145, 1999.

[242] Rijnkels MM. Multispecies comparison of the casein gene loci and evolution of casein gene family. Journal of Mammary Gland Biology and Neoplasia, 7(3):327$345,2002$.

[243] Pérez MDM and Calvo MM. Interaction of beta-lactoglobulin with retinol 
and fatty acids and its role as a possible biological function for this protein: a review. Journal of dairy science, 78(5):978-988, 1995.

[244] Garde JJ, Bell SCS, and Eperon ICI. Multiple forms of mRNA encoding human pregnancy-associated endometrial alpha 2-globulin, a beta-lactoglobulin homologue. Proceedings of the National Academy of Sciences of the United States of America, 88(6):2456-2460, 1991.

[245] Julkunen M, Seppälä M, and Jänne OA. Complete amino acid sequence of human placental protein 14: a progesterone-regulated uterine protein homologous to beta-lactoglobulins. Proceedings of the National Academy of Sciences of the United States of America, 85(23):8845-8849, 1988.

[246] Virtanen T, Kinnunen T, and Rytkönen-Nissinen M. Mammalian lipocalin allergens - insights into their enigmatic allergenicity. Clinical $\&$ Experimental Allergy, 42(4):494-504, 2011.

[247] Restani PP, Gaiaschi AA, Plebani AA, Beretta BB, Cavagni GG, Fiocchi AA, Poiesi CC, Velonà TT, Ugazio AGA, and Galli CLC. Cross-reactivity between milk proteins from different animal species. Clinical and experimental allergy : journal of the British Society for Allergy and Clinical Immunology, 29(7):997-1004, 1999.

[248] Olivier CE, Lorena SLS, Pavan CR, dos Santos RAPG, dos Santos Lima RP, Pinto DG, da Silva MD, and de Lima Zollner R. Is it just lactose intolerance? Allergy and Asthma Proceedings, 33(5):432-436, 2012.

[249] Bevilacqua C, Martin P, Candalh C, Fauquant J, Piot M, Roucayrol AM, Pilla F, and Heyman M. Goats' milk of defective alpha(s1)-casein genotype decreases intestinal and systemic sensitization to beta-lactoglobulin in guinea pigs. The Journal of dairy research, 68(2):217-227, 2001.

[250] Lara-Villoslada FF, Olivares MM, and Xaus JJ. The balance between caseins and whey proteins in cow's milk determines its allergenicity. Journal of dairy science, 88(5):1654-1660, 2005.

[251] Mishra A, Gaur SN, Singh BP, and Arora N. In silico assessment of the potential allergenicity of transgenes used for the development of GM food crops. Food and Chemical Toxicology, 50(5):1334-1339, 2012.

[252] Hileman RE, Silvanovich A, Goodman RE, Rice EA, Holleschak G, Astwood JD, and Hefle SL. Bioinformatic methods for allergenicity assessment using a comprehensive allergen database. International Archives of Allergy and Immunology, 128(4):280-291, 2002. 
[253] Brusic V and Petrovsky N. Bioinformatics for characterisation of allergens, allergenicity and allergic crossreactivity. Trends in Immunology, 24(5):225-228, 2003.

[254] Food and Agriculture Organization of the United Nations (FAO) Rome, Italy. Evaluation of allergenicity of Genetically Modified Foods. Tech. rep., Food and Agriculture Organization of the United Nations (FAO) Rome, Italy, 2001.

[255] Bauch C, Kolle SN, Ramirez T, Eltze T, Fabian E, Mehling A, Teubner W, van Ravenzwaay $\mathbf{B}$, and Landsiedel R. Putting the parts together: Combining in vitro methods to test for skin sensitizing potentials. Regulatory Toxicology and Pharmacology, 63(3):489-504, 2012.

[256] Järvinen KM, Beyer K, Vila L, Chatchatee P, Busse PJ, and Sampson HA. B-cell epitopes as a screening instrument for persistent cow's milk allergy. Journal of Allergy and Clinical Immunology, 110(2):293-297, 2002.

[257] Chatchatee P, Jarvinen KM, Bardina L, Vila L, Beyer K, and Sampson HA. Identification of $\operatorname{IgE}$ and $\operatorname{IgG}$ binding epitopes on beta- and kappacasein in cow's milk allergic patients. Clinical and experimental allergy : journal of the British Society for Allergy and Clinical Immunology, 31(8):1256-1262, 2001 .

[258] Pierson-Mullany LKL, Jackola DRD, Blumenthal MNM, and Rosenberg AA. Characterization of polyclonal allergen-specific $\operatorname{IgE}$ responses by affinity distributions. Molecular Immunology, 37(10):613-620, 2000.

[259] Clement G, Boquet D, Frobert Y, Bernard H, Negroni L, Chatel JM, Adel-Patient K, Creminon C, Wal JM, and Grassi J. Epitopic characterization of native bovine beta-lactoglobulin. Journal of immunological methods, 266(1-2):67-78, 2002.

[260] Pomés A. Relevant B Cell Epitopes in Allergic Disease. International Archives of Allergy and Immunology, 152(1):1-11, 2010.

[261] Niemi M, Jylhä S, Laukkanen ML, Söderlund H, Mäkinen-Kiljunen S, Kallio JM, Hakulinen N, Haahtela T, Takkinen K, and Rouvinen J. Molecular Interactions between a Recombinant IgE Antibody and the Lactoglobulin Allergen. Structure, 15(11):1413-1421, 2007.

[262] Hochwallner H, Schulmeister U, Swoboda I, Focke-Tejkl M, Civaj V, Balic N, Nystrand M, Härlin A, Thalhamer J, Scheiblhofer S, Keller W, Pavkov T, Zafred D, Niggemann B, Quirce S, Mari A, Pauli G, Ebner C, Papadopoulos NG, Herz U, van Tol EAF, Valenta R, and 
Spitzauer S. Visualization of clustered IgE epitopes on alpha-lactalbumin. The Journal of allergy and clinical immunology, 125(6):1279-1285.e9, 2010.

[263] Adel-Patient K, Bernard H, Ah-Leung S, Créminon C, and Wal JM. Peanut- and cow's milk-specific IgE, Th2 cells and local anaphylactic reaction are induced in Balb/c mice orally sensitized with cholera toxin. Allergy, 60(5):658-664, 2005.

[264] López-Expósito I, Chicón R, Belloque J, López-Fandiño R, and Berin MC. In vivo methods for testing allergenicity show that high hydrostatic pressure hydrolysates of b-lactoglobulin are immunologically inert. Journal of dairy science, 95(2):541-548, 2012.

[265] Li XM, Serebrisky D, Lee SY, Huang CK, Bardina L, Schofield BH, Stanley JS, Burks AW, Bannon GA, and Sampson HA. A murine model of peanut anaphylaxis: $\mathrm{T}$ - and B-cell responses to a major peanut allergen mimic human responses. Journal of Allergy and Clinical Immunology, 106(1):150-158, 2000 .

[266] Cunningham PT, Elliot CE, Lenzo JC, Jarnicki AG, Larcombe AN, Zosky GR, Holt PG, and Thomas WR. Sensitizing and Th2 adjuvant activity of cysteine protease allergens. International Archives of Allergy and Immunology, 158(4):347-358, 2012.

[267] Wang LF, Lin JY, Hsieh KH, and Lin RH. Epicutaneous exposure of protein antigen induces a predominant Th2-like response with high IgE production in mice. Journal of Immunology, 156(11):4077-4082, 1996.

[268] Morin S, Bernard H, Przybylski-Nicaise L, Corthier G, Rabot S, Wal JM, and Hazebrouck S. Allergenic and immunogenic potential of cow's milk -lactoglobulin and caseins evidenced without adjuvant in germ-free mice. Molecular Nutrition \& Food Research, 55(11):1700-1707, 2011.

[269] Vogel L, Luttkopf D, Hatahet L, Haustein D, and Vieths S. Development of a functional in vitro assay as a novel tool for the standardization of allergen extracts in the human system. Allergy, 60(8):1021-1028, 2005.

[270] Kimber I and Weisenberger C. A murine local lymph node assay for the identification of contact allergens. Assay development and results of an initial validation study. Archives of toxicology, 63(4):274-282, 1989.

[271] Belkaid Y, Kamhawi S, Modi G, Valenzuela J, Noben-Trauth N, Rowton E, Ribeiro J, and Sacks DL. Development of a natural model of cutaneous leishmaniasis: powerful effects of vector saliva and saliva preexposure on 
the long-term outcome of Leishmania major infection in the mouse ear dermis. The Journal of experimental medicine, 188(10):1941-1953, 1998.

[272] Van den Broeck W, Derore A, and Simoens P. Anatomy and nomenclature of murine lymph nodes: Descriptive study and nomenclatory standardization in BALB/cAnNCrl mice. Journal of immunological methods, 312(1-2):1219, 2006.

[273] Hu-Li J, Pannetier C, Guo L, Löhning M, Gu H, Watson C, Assenmacher M, Radbruch A, and Paul WE. Regulation of expression of IL-4 alleles: analysis using a chimeric GFP/IL-4 gene. Immunity, 14(1):1-11, 2001.

[274] Finkelman FD, Holmes J, Urban JF, Paul WE, and Katona IM. T help requirements for the generation of an in vivo $\operatorname{IgE}$ response: a late acting form of T cell help other than IL-4 is required for IgE but not for IgG1 production. Journal of Immunology, 142(2):403-408, 1989.

[275] Reinhardt RL, Liang HE, and Locksley RM. Cytokine-secreting follicular T cells shape the antibody repertoire. Nature Immunology, 10(4):385-393, 2009.

[276] Chvatchko Y, Kosco-Vilbois MH, Herren S, Lefort J, and Bonnefoy JY. Germinal center formation and local immunoglobulin E (IgE) production in the lung after an airway antigenic challenge. The Journal of experimental medicine, 184(6):2353-2360, 1996.

[277] Rangel-Moreno J, Moyron-Quiroz JE, Carragher DM, Kusser K, Hartson L, Moquin A, and Randall TD. Omental Milky Spots Develop in the Absence of Lymphoid Tissue-Inducer Cells and Support B and T Cell Responses to Peritoneal Antigens. Immunity, 30(5):731-743, 2009.

[278] Yu D and Vinuesa CG. The elusive identity of T follicular helper cells. Trends in Immunology, 31(10):377-383, 2010.

[279] Erazo A, Kutchukhidze N, Leung M, Christ APG, Urban Jr JF, Curotto de Lafaille MA, and Lafaille JJ. Unique Maturation Program of the IgE Response In Vivo. Immunity, 26(2):191-203, 2007.

[280] Craig SW and Cebra JJ. Peyer's patches: an enriched source of precursors for IgA-producing immunocytes in the rabbit. The Journal of experimental medicine, 134(1):188-200, 1971.

[281] McHeyzer-Williams M, Okitsu S, Wang N, and McHeyzer-Williams L. Molecular programming of B cell memory. Nature Reviews Immunology, 12(1):24-34, 2011. 
[282] Lorenz M, Jung S, and Radbruch A. Switch transcripts in immunoglobulin class switching. Science (New York, N.Y.), 267(5205):1825-1828, 1995.

[283] Stavnezer-Nordgren J and Sirlin S. Specificity of immunoglobulin heavy chain switch correlates with activity of germline heavy chain genes prior to switching. The EMBO journal, 5(1):95-102, 1986.

[284] Brinkmann VV and Heusser CHC. T cell-dependent differentiation of human B cells into $\operatorname{IgM}, \operatorname{IgG}$, IgA, or IgE plasma cells: high rate of antibody production by IgE plasma cells, but limited clonal expansion of IgE precursors. Cellular Immunology, 152(2):323-332, 1993.

[285] Snapper CM and Paul WE. Interferon-gamma and B cell stimulatory factor-1 reciprocally regulate Ig isotype production. Science (New York, N.Y.), 236(4804):944-947, 1987.

[286] Pène J, Rousset F, Brière F, Chrétien I, Bonnefoy JY, Spits H, Yokota T, Arai N, Arai K, and Banchereau J. IgE production by normal human lymphocytes is induced by interleukin 4 and suppressed by interferons gamma and alpha and prostaglandin E2. Proceedings of the National Academy of Sciences of the United States of America, 85(18):6880-6884, 1988.

[287] Finkelman FD, Katona IM, Urban JF, Holmes J, Ohara J, Tung AS, Sample JV, and Paul WE. IL-4 is required to generate and sustain in vivo IgE responses. Journal of Immunology, 141(7):2335-2341, 1988.

[288] Finkelman FD, Holmes J, Katona IM, Urban JF, Beckmann MP, Park LS, Schooley KA, Coffman RL, Mosmann TR, and Paul WE. Lymphokine control of in vivo immunoglobulin isotype selection. Annual review of immunology, 8:303-333, 1990.

[289] Kopf M, Le Gros G, Bachmann M, Lamers MC, Bluethmann H, and Kohler G. Disruption of the Murine Il-4 Gene Blocks Th2 Cytokine Responses. Nature, 362(6417):245-248, 1993.

[290] Xu J, Foy TM, Laman JD, Elliott EA, Dunn JJ, Waldschmidt TJ, Elsemore J, Noelle RJ, and Flavell RA. Mice deficient for the CD40 ligand. Immunity, 1(5):423-431, 1994.

[291] Kawabe T, Naka T, Yoshida K, Tanaka T, Fujiwara H, Suematsu S, Yoshida N, Kishimoto $\mathbf{T}$, and Kikutani $\mathbf{H}$. The immune responses in CD40-deficient mice: impaired immunoglobulin class switching and germinal center formation. Immunity, 1(3):167-178, 1994.

[292] Gauchat JF, Henchoz S, Mazzei G, Aubry JP, Brunner T, Blasey H, 
Life P, Talabot D, Flores-Romo L, and Thompson J. Induction of human IgE synthesis in B cells by mast cells and basophils. Nature, 365(6444):340-343, 1993.

[293] Yamaguchi M, Sayama K, Yano K, Lantz CS, Noben-Trauth N, Ra C, Costa JJ, and Galli SJ. IgE enhances Fc epsilon receptor I expression and IgE-dependent release of histamine and lipid mediators from human umbilical cord blood-derived mast cells: synergistic effect of IL-4 and $\operatorname{IgE}$ on human mast cell Fc epsilon receptor I expression and mediator release. Journal of Immunology, 162(9):5455-5465, 1999.

[294] Corry DB, Folkesson HG, Warnock ML, Erle DJ, Matthay MA, Wiener-Kronish JP, and Locksley RM. Interleukin 4, but not interleukin 5 or eosinophils, is required in a murine model of acute airway hyperreactivity. The Journal of experimental medicine, 183(1):109-117, 1996.

[295] Brandt EB, Munitz A, Orekov T, Mingler MK, McBride M, Finkelman FD, and Rothenberg ME. Targeting IL-4/IL-13 signaling to alleviate oral allergen-induced diarrhea. Journal of Allergy and Clinical Immunology, 123(1):53-58, 2009.

[296] Robinson DS, Hamid Q, Ying S, Tsicopoulos A, Barkans J, Bentley AM, Corrigan C, Durham SR, and Kay AB. Predominant TH2-like bronchoalveolar T-lymphocyte population in atopic asthma. The New England journal of medicine, 326(5):298-304, 1992.

[297] Hogan SP, Mishra A, Brandt EB, Foster PS, and Rothenberg ME. A critical role for eotaxin in experimental oral antigen-induced eosinophilic gastrointestinal allergy. Proceedings of the National Academy of Sciences of the United States of America, 97(12):6681-6686, 2000.

[298] Marinaro M, Staats HF, Hiroi T, Jackson RJ, Coste M, Boyaka PN, Okahashi N, Yamamoto M, Kiyono H, Bluethmann H, Fujihashi K, and McGhee JR. Mucosal adjuvant effect of cholera toxin in mice results from induction of T helper 2 (Th2) cells and IL-4. Journal of Immunology, 155(10):4621-4629, 1995.

[299] Blazquez AB, Knight AK, Getachew H, Bromberg JS, Lira SA, Mayer L, and Berin MC. A Functional Role for CCR6 on Proallergic T Cells in the Gastrointestinal Tract. Gastroenterology, 138(1):275-284.e4, 2010.

[300] Min B. Basophils Produce IL-4 and Accumulate in Tissues after Infection with a Th2-inducing Parasite. Journal of Experimental Medicine, 200(4):507-517, 2004 . 
[301] Lantz CS, Min B, Tsai M, Chatterjea D, Dranoff G, and Galli SJ. IL-3 is required for increases in blood basophils in nematode infection in mice and can enhance IgE-dependent IL-4 production by basophils in vitro. Laboratory Investigation, 88(11):1134-1142, 2008.

[302] Voehringer D, Shinkai K, and Locksley RM. Type 2 immunity reflects orchestrated recruitment of cells committed to IL-4 production. Immunity, 20(3):267-277, 2004.

[303] Wu WH, Park CO, Oh SH, Kim HJ, Kwon YS, Bae BG, Noh JY, and Lee KH. Thymic stromal lymphopoietin-activated invariant natural killer $\mathrm{T}$ cells trigger an innate allergic immune response in atopic dermatitis. The Journal of allergy and clinical immunology, 126(2):290-299.e4, 2010.

[304] Pawankar R, Yamagishi S, and Yagi T. Revisiting the roles of mast cells in allergic rhinitis and its relation to local IgE synthesis. American journal of rhinology, 14(5):309-317, 2000.

[305] Harris DP, Haynes L, Sayles PC, Duso DK, Eaton SM, Lepak NM, Johnson LL, Swain SL, and Lund FE. Reciprocal regulation of polarized cytokine production by effector B and T cells. Nature Immunology, 1(6):475$482,2000$.

[306] Xiong H, Dolpady J, Wabl M, Curotto de Lafaille MA, and Lafaille JJ. Sequential class switching is required for the generation of high affinity $\operatorname{IgE}$ antibodies. Journal of Experimental Medicine, 209(2):353-364, 2012.

[307] Kimura K, Song CH, Rastogi A, Dranoff G, Galli SJ, and Lantz CS. Interleukin-3 and c-Kit/stem cell factor are required for normal eosinophil responses in mice infected with Strongyloides venezuelensis. Laboratory Investigation, 86(10):987-996, 2006.

[308] Berin MC, Kiliaan AJ, Yang PC, Groot JA, Taminiau JA, and Perdue MH. Rapid transepithelial antigen transport in rat jejunum: impact of sensitization and the hypersensitivity reaction. Gastroenterology, 113(3):856864, 1997.

[309] Lantz CS, Boesiger J, Song CH, Mach N, Kobayashi T, Mulligan RC, Nawa Y, Dranoff G, and Galli SJ. Role for interleukin-3 in mast-cell and basophil development and in immunity to parasites. Nature, 392(6671):90-93, 1998.

[310] Osterfeld H, Ahrens R, Strait R, Finkelman FD, Renauld JC, and Hogan SP. Differential roles for the IL-9/IL-9 receptor \&alpha;-chain path- 
way in systemic and oral antigen-induced anaphylaxis. Journal of Allergy and Clinical Immunology, 125(2):469-476.e2, 2010.

[311] Abe T, Sugaya H, Ishida K, Khan WI, Tasdemir I, and Yoshimura K. Intestinal protection against Strongyloides ratti and mastocytosis induced by administration of interleukin-3 in mice. Immunology, 80(1):116-121, 1993.

[312] Faulkner H, Renauld JC, Van Snick J, and Grencis RK. Interleukin-9 enhances resistance to the intestinal nematode Trichuris muris. Infection and immunity, 66(8):3832-3840, 1998.

[313] Urban JF, Noben-Trauth N, Donaldson DD, Madden KB, Morris SC, Collins M, and Finkelman FD. IL-13, IL-4Ralpha, and Stat6 are required for the expulsion of the gastrointestinal nematode parasite Nippostrongylus brasiliensis. Immunity, 8(2):255-264, 1998.

[314] Gurish MF and Boyce JA. Mast cell growth, differentiation, and death. Clinical reviews in allergy \& immunology, 22(2):107-118, 2002.

[315] Svetic A, Madden KB, Zhou XD, Lu P, Katona IM, Finkelman FD, Urban JF, and Gause WC. A primary intestinal helminthic infection rapidly induces a gut-associated elevation of Th2-associated cytokines and IL-3. Journal of Immunology, 150(8 Pt 1):3434-3441, 1993.

[316] Shen T, Kim S, Do Js, Wang L, Lantz C, Urban JF, Le Gros G, and Min B. T cell-derived IL-3 plays key role in parasite infection-induced basophil production but is dispensable for in vivo basophil survival. International Immunology, 20(9):1201-1209, 2008.

[317] Liang HE, Reinhardt RL, Bando JK, Sullivan BM, Ho IC, and Locksley RM. Divergent expression patterns of IL-4 and IL-13 define unique functions in allergic immunity. Nature Publishing Group, pp. 1-11, 2011.

[318] Behnke JM, Wahid FN, Grencis RK, Else KJ, Ben-Smith AW, and Goyal PK. Immunological relationships during primary infection with Heligmosomoides polygyrus (Nematospiroides dubius): downregulation of specific cytokine secretion (IL-9 and IL-10) correlates with poor mastocytosis and chronic survival of adult worms. Parasite Immunology, 15(7):415-421, 1993.

[319] Ventura MT, Polimeno L, Amoruso AC, Gatti F, Annoscia E, Marinaro M, Di Leo E, Matino MG, Buquicchio R, Bonini S, Tursi A, and Francavilla A. Intestinal permeability in patients with adverse reactions to food. Digestive and liver disease : official journal of the Italian Society of Gastroenterology and the Italian Association for the Study of the Liver, 38(10):732736, 2006. 
[320] Berin MC, Kiliaan AJ, Yang PC, Groot JA, Kitamura Y, and Perdue MH. The influence of mast cells on pathways of transepithelial antigen transport in rat intestine. Journal of Immunology, 161(5):2561-2566, 1998.

[321] Brandt EB, Scribner TA, Saito Akei H, and Rothenberg ME. Experimental gastrointestinal allergy enhances pulmonary responses to specific and unrelated allergens. Journal of Allergy and Clinical Immunology, 118(2):420427, 2006.

[322] Mosmann TR, Cherwinski H, Bond MW, Giedlin MA, and Coffman RL. Two types of murine helper T cell clone. I. Definition according to profiles of lymphokine activities and secreted proteins. Journal of Immunology, 136(7):2348-2357, 1986.

[323] Harvie M, Camberis M, Tang SC, Delahunt B, Paul W, and Le Gros G. The Lung Is an Important Site for Priming CD4 T-Cell-Mediated Protective Immunity against Gastrointestinal Helminth Parasites. Infection and immunity, 78(9):3753-3762, 2010.

[324] Kanno Y, Vahedi G, Hirahara K, Singleton K, and O'Shea JJ. Transcriptional and Epigenetic Control of T Helper Cell Specification: Molecular Mechanisms Underlying Commitment and Plasticity *. Annual review of immunology, 30(1):707-731, 2012.

[325] Paul WE. What determines Th2 differentiation, in vitro and in vivo? Immunology and Cell Biology, pp. 1-4, 2010.

[326] Goswami R, Jabeen R, Yagi R, Pham D, Zhu J, Goenka S, and Kaplan MH. STAT6-Dependent Regulation of Th9 Development. The Journal of Immunology, 188(3):968-975, 2012.

[327] Nakamura T, Kamogawa Y, Bottomly K, and Flavell RA. Polarization of IL-4- and IFN-gamma-producing CD4+ T cells following activation of naive CD4+ T cells. Journal of Immunology, 158(3):1085-1094, 1997.

[328] Zheng W and Flavell RA. The transcription factor GATA-3 is necessary and sufficient for Th2 cytokine gene expression in CD4 T cells. Cell, 89(4):587-596, 1997.

[329] Kaplan MH, Schindler U, Smiley ST, and Grusby MJ. Stat6 is required for mediating responses to IL-4 and for development of Th2 cells. Immunity, 4(3):313-319, 1996.

[330] Takeda K, Tanaka T, Shi W, Matsumoto M, Minami M, Kashiwamura 
S, Nakanishi K, Yoshida N, Kishimoto T, and Akira S. Essential role of Stat6 in IL-4 signalling. Nature, 380(6575):627-630, 1996.

[331] Shimoda K, van Deursen J, Sangster MY, Sarawar SR, Carson RT, Tripp RA, Chu C, Quelle FW, Nosaka T, Vignali DA, Doherty PC, Grosveld G, Paul WE, and Ihle JN. Lack of IL-4-induced Th2 response and IgE class switching in mice with disrupted Stat6 gene. Nature, 380(6575):630 633, 1996.

[332] Yagi R, Junttila IS, Wei G, Urban Jr JF, Zhao K, Paul WE, and Zhu J. The Transcription Factor GATA3 Actively Represses RUNX3 ProteinRegulated Production of Interferon-\&gamma;. Immunity, 32(4):507-517, 2010.

[333] Szabo SJ, Kim ST, Costa GL, Zhang X, Fathman CG, and Glimcher LH. A novel transcription factor, T-bet, directs Th1 lineage commitment. Cell, 100(6):655-669, 2000.

[334] Perona-Wright G, Mohrs K, and Mohrs M. Sustained signaling by canonical helper T cell cytokines throughout the reactive lymph node. Nature Publishing Group, 11(6):520-526, 2010.

[335] Ivanov II, McKenzie BS, Zhou L, Tadokoro CE, Lepelley A, Lafaille JJ, Cua DJ, and Littman DR. The Orphan Nuclear Receptor RORt Directs the Differentiation Program of Proinflammatory IL-17+ T Helper Cells. Cell, 126(6):1121-1133, 2006.

[336] Zhou L, Lopes JE, Chong MMW, Ivanov II, Min R, Victora GD, Shen Y, Du J, Rubtsov YP, Rudensky AY, Ziegler SF, and Littman DR. TGF--induced Foxp3 inhibits TH17 cell differentiation by antagonizing RORt function. Nature, 453(7192):236-240, 2008.

[337] Haynes NM, Allen CDC, Lesley R, Ansel KM, Killeen N, and Cyster JG. Role of CXCR5 and CCR7 in follicular Th cell positioning and appearance of a programmed cell death gene-1high germinal center-associated subpopulation. Journal of Immunology, 179(8):5099-5108, 2007.

[338] Linterman MA, Rigby RJ, Wong RK, Yu D, Brink R, Cannons JL, Schwartzberg PL, Cook MC, Walters GD, and Vinuesa CG. Follicular helper T cells are required for systemic autoimmunity. Journal of Experimental Medicine, 206(3):561-576, 2009.

[339] Johnston RJ, Poholek AC, DiToro D, Yusuf I, Eto D, Barnett B, Dent AL, Craft J, and Crotty S. Bcl6 and Blimp-1 Are Reciprocal and Antagonistic Regulators of T Follicular Helper Cell Differentiation. Science (New York, N.Y.), 325(5943):1006-1010, 2009. 
[340] Nurieva RI, Chung Y, Martinez GJ, Yang XO, Tanaka S, Matskevitch TD, Wang YH, and Dong C. Bcl6 Mediates the Development of T Follicular Helper Cells. Science (New York, N.Y.), 325(5943):1001-1005, 2009.

[341] Yu D, Rao S, Tsai LM, Lee SK, He Y, Sutcliffe EL, Srivastava M, Linterman M, Zheng L, Simpson N, Ellyard JI, Parish IA, Ma CS, Li QJ, Parish CR, Mackay CR, and Vinuesa CG. The Transcriptional Repressor Bcl-6 Directs T Follicular Helper Cell Lineage Commitment. Immunity, 31(3):457-468, 2009.

[342] Le Gros G, Ben-Sasson SZ, Seder R, Finkelman FD, and Paul WE. Generation of interleukin 4 (IL-4)-producing cells in vivo and in vitro: IL-2 and IL-4 are required for in vitro generation of IL-4-producing cells (Reprinted from The Journal of Experimental Medicine, vol 172, pg 921-929, 1990). Journal of Immunology, 181(5):2943-2951, 2008.

[343] Swain SL, Weinberg AD, English M, and Huston G. IL-4 directs the development of Th2-like helper effectors. Journal of Immunology, 145(11):37963806, 1990.

[344] Swain SL, Weinberg AD, and English M. CD4+ T cell subsets. Lymphokine secretion of memory cells and of effector cells that develop from precursors in vitro. Journal of Immunology, 144(5):1788-1799, 1990.

[345] Campbell SB, Komata T, and Kelso A. CD4 ligation promotes the IL-4independent development of IL-4-producing clones from naive CD4 $(+) \mathrm{T}$ cells. Journal of Immunology, 167(10):5610-5619, 2001.

[346] Komata T, Cruikshank WW, and Kelso A. Sustained linked stimulation via CD3 and CD4 is required for the IL-4-independent development of IL-4 synthesizing CD4+ T cells. Immunology and Cell Biology, 81(4):283-288, 2003.

[347] Grogan JL, Mohrs M, Harmon B, Lacy DA, Sedat JW, and Locksley RM. Early transcription and silencing of cytokine genes underlie polarization of T helper cell subsets. Immunity, 14(3):205-215, 2001.

[348] Ouyang W, Löhning M, Gao Z, Assenmacher M, Ranganath S, Radbruch A, and Murphy KM. Stat6-independent GATA-3 autoactivation directs IL-4-independent Th2 development and commitment. Immunity, 12(1):2737,2000 .

[349] Yamane H. Independent roles for IL-2 and GATA-3 in stimulating naive CD4+ T cells to generate a Th2-inducing cytokine environment. Journal of Experimental Medicine, 202(6):793-804, 2005. 
[350] Kamogawa Y, Minasi LA, Carding SR, Bottomly K, and Flavell RA. The relationship of IL-4- and IFN gamma-producing T cells studied by lineage ablation of IL-4-producing cells. Cell, 75(5):985-995, 1993.

[351] Ito T. TSLP-activated dendritic cells induce an inflammatory T helper type 2 cell response through OX40 ligand. Journal of Experimental Medicine, 202(9):1213-1223, 2005.

[352] Scheu S, Stetson DB, Reinhardt RL, Leber JH, Mohrs M, and Locksley RM. Activation of the integrated stress response during $\mathrm{T}$ helper cell differentiation. Nature Immunology, 7(6):644-651, 2006.

[353] Sokol CL, Chu NQ, Yu S, Nish SA, Laufer TM, and Medzhitov R. Basophils function as antigen-presenting cells for an allergen-induced $\mathrm{T}$ helper type 2 response. Nature Publishing Group, 10(7):713-721, 2009.

[354] Yoshimoto T, Yasuda K, Tanaka H, Nakahira M, Imai Y, Fujimori Y, and Nakanishi K. Basophils contribute to TH2-IgE responses in vivo via IL-4 production and presentation of peptide-MHC class II complexes to CD4+ T cells. Nature Publishing Group, 10(7):706-712, 2009.

[355] Saenz SA, Siracusa MC, Perrigoue JG, Spencer SP, Urban JF, Tocker JE, Budelsky AL, Kleinschek MA, Kastelein RA, Kambayashi T, Bhandoola A, and Artis D. IL25 elicits a multipotent progenitor cell population that promotes TH2 cytokine responses. Nature, pp. 1-6, 2010.

[356] Angkasekwinai P, Park H, Wang YH, Wang YH, Chang SH, Corry DB, Liu YJ, Zhu Z, and Dong C. Interleukin 25 promotes the initiation of proallergic type 2 responses. Journal of Experimental Medicine, 204(7):15091517, 2007.

[357] van Panhuys N, Prout M, Forbes E, Min B, Paul WE, and Le Gros G. Basophils Are the Major Producers of IL-4 during Primary Helminth Infection. The Journal of Immunology, 186(5):2719-2728, 2011.

[358] Sullivan BM, Liang HE, Bando JK, Wu D, Cheng LE, McKerrow JK, Allen CDC, and Locksley RM. Genetic analysis of basophil function in vivo. Nature Immunology, 12(6):527-535, 2011.

[359] Hammad H, Plantinga M, Deswarte K, Pouliot P, Willart MAM, Kool M, Muskens F, and Lambrecht BN. Inflammatory dendritic cells-not basophils-are necessary and sufficient for induction of Th2 immunity to inhaled house dust mite allergen. Journal of Experimental Medicine, 207(10):2097-2111, 2010 . 
[360] Cunningham AF, Serre K, Toellner KM, Khan M, Alexander J, Brombacher F, and MacLennan ICM. Pinpointing IL-4-independent acquisition and IL-4-influenced maintenance of Th2 activity by CD4 T cells. European journal of immunology, 34(3):686-694, 2004.

[361] Cunningham AF and Toellner KM. Rapid development of Th2 activity during T cell priming. Clinical \&3 developmental immunology, 10(1):1-6, 2003.

[362] King IL and Mohrs M. IL-4-producing CD4+ T cells in reactive lymph nodes during helminth infection are $\mathrm{T}$ follicular helper cells. Journal of Experimental Medicine, 206(5):1001-1007, 2009.

[363] Noben-Trauth N, Shultz LD, Brombacher F, Urban JF, Gu H, and Paul WE. An interleukin 4 (IL-4)-independent pathway for CD4+ T cell IL-4 production is revealed in IL-4 receptor-deficient mice. Proceedings of the National Academy of Sciences of the United States of America, 94(20):1083810843, 1997.

[364] Zaretsky AG, Taylor JJ, King IL, Marshall FA, Mohrs M, and Pearce EJ. T follicular helper cells differentiate from Th2 cells in response to helminth antigens. Journal of Experimental Medicine, 206(5):991-999, 2009.

[365] Hathcock KS, Pucillo CE, Laszlo G, Lai L, and Hodes RJ. Analysis of thymic subpopulations expressing the activation antigen GL7. Expression, genetics, and function. Journal of Immunology, 155(10):4575-4581, 1995.

[366] Yusuf I, Kageyama R, Monticelli L, Johnston RJ, Ditoro D, Hansen K, Barnett B, and Crotty S. Germinal center T follicular helper cell IL-4 production is dependent on signaling lymphocytic activation molecule receptor (CD150). The Journal of Immunology, 185(1):190-202, 2010.

[367] Luthje K, Kallies A, Shimohakamada Y, Belz GT, Light A, Tarlinton DM, and Nutt SL. The development and fate of follicular helper T cells defined by an IL-21 reporter mouse. Nature Publishing Group, pp. 1-9, 2012.

[368] Choi YS, Kageyama R, Eto D, Escobar TC, Johnston RJ, Monticelli L, Lao C, and Crotty S. ICOS Receptor Instructs T Follicular Helper Cell versus Effector Cell Differentiation via Induction of the Transcriptional Repressor Bcl6. Immunity, pp. 1-15, 2011.

[369] Fazilleau N, McHeyzer-Williams LJ, Rosen H, and McHeyzerWilliams MG. The function of follicular helper $\mathrm{T}$ cells is regulated by the strength of T cell antigen receptor binding. Nature Immunology, 10(4):375-384, 2009 . 
[370] Baumgarth N and Kelso A. Functionally distinct T cells in three compartments of the respiratory tract after influenza virus infection. European journal of immunology, 26(9):2189-2197, 1996.

[371] Finkelman FD and Morris SC. Development of an assay to measure in vivo cytokine production in the mouse. International Immunology, 11(11):1811-1818, 1999.

[372] Mohrs M, Shinkai K, Mohrs K, and Locksley RM. Analysis of type 2 immunity in vivo with a bicistronic IL-4 reporter. Immunity, 15(2):303-311, 2001.

[373] Mohrs K, Wakil AE, Killeen N, Locksley RM, and Mohrs M. A TwoStep Process for Cytokine Production Revealed by IL-4 Dual-Reporter Mice. Immunity, 23(4):419-429, 2005.

[374] Rivière I, Sunshine MJ, and Littman DR. Regulation of IL-4 expression by activation of individual alleles. Immunity, 9(2):217-228, 1998.

[375] Sawada S, Scarborough JD, Killeen N, and Littman DR. A lineagespecific transcriptional silencer regulates CD4 gene expression during T lymphocyte development. Cell, 77(6):917-929, 1994.

[376] Gett AV and Hodgkin PD. Cell division regulates the T cell cytokine repertoire, revealing a mechanism underlying immune class regulation. Proceedings of the National Academy of Sciences of the United States of America, 95(16):94889493, 1998.

[377] Allan RS, Zueva E, Cammas F, Schreiber HA, Masson V, Belz GT, Roche D, Maison C, Quivy JP, Almouzni G, and Amigorena S. An epigenetic silencing pathway controlling $\mathrm{T}$ helper 2 cell lineage commitment. Nature, pp. 1-6, 2012.

[378] Bird JJ, Brown DR, Mullen AC, Moskowitz NH, Mahowald MA, Sider JR, Gajewski TF, Wang CR, and Reiner SL. Helper T cell differentiation is controlled by the cell cycle. Immunity, 9(2):229-237, 1998.

[379] Agarwal S and Rao A. Modulation of chromatin structure regulates cytokine gene expression during T cell differentiation. Immunity, 9(6):765-775, 1998.

[380] Ansel KM, Greenwald RJ, Agarwal S, Bassing CH, Monticelli S, Interlandi J, Djuretic IM, Lee DU, Sharpe AH, Alt FW, and Rao A. Deletion of a conserved Il4 silencer impairs T helper type 1-mediated immunity. Nature Immunology, 5(12):1251-1259, 2004.

[381] Guo L, Hu-Li J, and Paul WE. Probabilistic Regulation in TH2 Cells 
Accounts for Monoallelic Expression of IL-4 and IL-13. Immunity, 23(1):89-99, 2005.

[382] Lee DU, Agarwal S, and Rao A. Th2 lineage commitment and efficient IL-4 production involves extended demethylation of the IL-4 gene. Immunity, 16(5):649-660, 2002.

[383] Lee GR, Fields PE, Griffin IV TJ, and Flavell RA. Regulation of the Th2 Cytokine Locus by a Locus Control Region. Immunity, 19(1):145-153, 2003.

[384] Tanaka S, Motomura Y, Suzuki Y, Yagi R, Inoue H, Miyatake S, and Kubo M. The enhancer HS2 critically regulates GATA-3-mediated Il4 transcription in TH2 cells. Nature Immunology, 12(1):77-85, 2010.

[385] Ansel KM, Djuretic I, Tanasa B, and Rao A. Regulation of Th2 differentiation and Il4 locus accessibility. Annual review of immunology, 24:607-656, 2006.

[386] Duffy KR, Wellard CJ, Markham JF, Zhou JHS, Holmberg R, Hawkins ED, Hasbold J, Dowling MR, and Hodgkin PD. ActivationInduced B Cell Fates Are Selected by Intracellular Stochastic Competition. Science (New York, N.Y.), 335(6066):338-341, 2012.

[387] Ross IL, Browne CM, and Hume DA. Transcription of individual genes in eukaryotic cells occurs randomly and infrequently. Immunology and Cell Biology, 72(2):177-185, 1994.

[388] Mariani L, Schulz EG, Lexberg MH, Helmstetter C, Radbruch A, hning MLo, and fer THo. Short-term memory in gene induction reveals the regulatory principle behind stochastic IL-4 expression. Molecular Systems Biology, 6:1-13, 2010.

[389] Bix M and Locksley RM. Independent and epigenetic regulation of the interleukin-4 alleles in CD4+ T cells. Science (New York, N.Y.), 281(5381):1352-1354, 1998.

[390] Bix M, Wang ZE, Thiel B, Schork NJ, and Locksley RM. Genetic regulation of commitment to interleukin 4 production by a CD4(+) $\mathrm{T}$ cellintrinsic mechanism. The Journal of experimental medicine, 188(12):2289-2299, 1998.

[391] Naramura M, Hu RJ, and Gu H. Mice with a fluorescent marker for interleukin 2 gene activation. Immunity, 9(2):209-216, 1998.

[392] Vijayanand P, Seumois G, Simpson LJ, Abdul-Wajid S, Baumjohann D, Panduro M, Huang X, Interlandi J, Djuretic IM, Brown DR, 
Sharpe AH, Rao A, and Ansel KM. Interleukin-4 Production by Follicular Helper T Cells Requires the Conserved Il4 Enhancer Hypersensitivity Site V. Immunity, pp. 1-13, 2012.

[393] Camberis M, Le Gros G, and Urban J. Animal model of Nippostrongylus brasiliensis and Heligmosomoides polygyrus. Current protocols in immunology / edited by John E. Coligan ... [et al.], Chapter 19:Unit 19.12, 2003.

[394] Hodgkinson AJ, McDonald NA, Kivits LJ, Hurford DR, Fahey S, and Prosser C. Allergic responses induced by goat milk S1-casein in a murine model of gastrointestinal atopy. Journal of dairy science, 95(1):83-90, 2012.

[395] Gillessen S. Overlapping roles for granulocyte-macrophage colony-stimulating factor and interleukin-3 in eosinophil homeostasis and contact hypersensitivity. Blood, 97(4):922-928, 2001.

[396] Mach N, Lantz CS, Galli SJ, Reznikoff G, Mihm M, Small C, Granstein R, Beissert S, Sadelain M, Mulligan RC, and Dranoff G. Involvement of interleukin-3 in delayed-type hypersensitivity. Blood, 91(3):778783, 1998.

[397] Alexander LJL, Hayes GG, Pearse MJM, Beattie CWC, Stewart AFA, Willis IMI, and Mackinlay AGA. Complete sequence of the bovine beta-lactoglobulin cDNA. Nucleic acids research, 17(16):6739-6739, 1989.

[398] Boratyn GM, ffer AAS, Agarwala R, Altschul SF, Lipman DJ, and Madden TL. Domain enhanced lookup time accelerated BLAST. Biology Direct, 7(1):1-1, 2012.

[399] Dialynas DP, Quan ZS, Wall KA, Pierres A, Quintáns J, Loken MR, Pierres M, and Fitch FW. Characterization of the murine T cell surface molecule, designated L3T4, identified by monoclonal antibody GK1.5: similarity of L3T4 to the human Leu-3/T4 molecule. Journal of Immunology, 131(5):24452451, 1983.

[400] Kruisbeek AM. In vivo depletion of CD4- and CD8-specific T cells. Current protocols in immunology / edited by John E. Coligan ... [et al.], Chapter 4:Unit 4.1, 2001.

[401] Guo L, Hu-Li J, and Paul WE. Probabilistic Regulation of IL-4 Production. Journal of Clinical Immunology, 25(6):573-581, 2005.

[402] Holländer GA, Zuklys S, Morel C, Mizoguchi E, Mobisson K, Simpson S, Terhorst C, Wishart W, Golan DE, Bhan AK, and Burakoff SJ. 
Monoallelic expression of the interleukin-2 locus. Science (New York, N.Y.), 279(5359):2118-2121, 1998.

[403] Guo L, Hu-Li J, and Paul WE. Probabilistic regulation of IL-4 production in Th2 cells: accessibility at the Il4 locus. Immunity, 20(2):193-203, 2004.

[404] Chan LSL, Robinson NN, and Xu LL. Expression of interleukin-4 in the epidermis of transgenic mice results in a pruritic inflammatory skin disease: an experimental animal model to study atopic dermatitis. Journal of Investigative Dermatology, 117(4):977-983, 2001.

[405] Erb KJK, Rüger BB, von Brevern MM, Ryffel BB, Schimpl AA, and Rivett KK. Constitutive expression of interleukin (IL)-4 in vivo causes autoimmune-type disorders in mice. The Journal of experimental medicine, 185(2):329-339, 1997.

[406] Gubin MM, Calaluce R, Davis JW, Magee JD, Strouse CS, Shaw DP, Ma L, Brown A, Hoffman T, Rold TL, and Atasoy U. Overexpression of the RNA binding protein $\mathrm{HuR}$ impairs tumor growth in triple negative breast cancer associated with deficient angiogenesis. Cell Cycle, 9(16):3337-3346, 2010.

[407] Le Gros G, Schultze N, Walti S, Einsle K, Finkelman F, Kosco-Vilbois MH, and Heusser C. The development of IgE+ memory B cells following primary IgE immune responses. European journal of immunology, 26(12):30423047, 1996.

[408] Cunningham AF, Fallon PG, Khan M, Vacheron S, Acha-Orbea H, MacLennan ICM, McKenzie AN, and Toellner KM. Th2 activities induced during virgin $\mathrm{T}$ cell priming in the absence of IL-4, IL-13, and B cells. Journal of Immunology, 169(6):2900-2906, 2002.

[409] Arnold CN, Campbell DJ, Lipp M, and Butcher EC. The germinal center response is impaired in the absence of T cell-expressed CXCR5. European journal of immunology, 37(1):100-109, 2007.

[410] Breitfeld D, Ohl L, Kremmer E, Ellwart J, Sallusto F, Lipp M, and Forster R. Follicular B helper T cells express CXC chemokine receptor 5, localize to B cell follicles, and support immunoglobulin production. The Journal of experimental medicine, 192(11):1545-1552, 2000.

[411] Bossaller L, Burger J, Draeger R, Grimbacher B, Knoth R, Plebani A, Durandy A, Baumann U, Schlesier M, Welcher AA, Peter HH, and Warnatz $\mathbf{K}$. ICOS deficiency is associated with a severe reduction of CXCR5+CD4 germinal center Th cells. Journal of Immunology, 177(7):49274932, 2006. 
[412] Banchereau J, Bazan F, Blanchard D, Brière F, Galizzi JP, van Kooten C, Liu YJ, Rousset F, and Saeland S. The CD40 antigen and its ligand. Annual review of immunology, 12:881-922, 1994.

[413] Cunningham AF, Serre K, Mohr E, Khan M, and Toellner KM. Loss of CD154 impairs the Th2 extrafollicular plasma cell response but not early $\mathrm{T}$ cell proliferation and interleukin-4 induction. Immunology, 113(2):187-193, 2004 .

[414] Korthäuer U, Graf D, Mages HW, Brière F, Padayachee M, Malcolm S, Ugazio AG, Notarangelo LD, Levinsky RJ, and Kroczek RA. Defective expression of T-cell CD40 ligand causes X-linked immunodeficiency with hyper-IgM. Nature, 361(6412):539-541, 1993.

[415] Finkelman FD, Katona IM, Mosmann TR, and Coffman RL. IFNgamma regulates the isotypes of Ig secreted during in vivo humoral immune responses. Journal of Immunology, 140(4):1022-1027, 1988.

[416] Grunewald SM, Teufel M, Erb K, Nelde A, Mohrs M, Brombacher F, Bröcker EB, Sebald W, and Duschl A. Upon prolonged allergen exposure IL-4 and IL-4Ralpha knockout mice produce specific IgE leading to anaphylaxis. International Archives of Allergy and Immunology, 125(4):322-328, 2001.

[417] McCoy KD, Harris NL, Diener P, Hatak S, Odermatt B, Hangartner L, Senn BM, Marsland BJ, Geuking MB, Hengartner H, Macpherson AJS, and Zinkernagel RM. Natural IgE Production in the Absence of MHC Class II Cognate Help. Immunity, 24(3):329-339, 2006.

[418] Fish SC, Donaldson DD, Goldman SJ, Williams CMM, and Kasaian MT. IgE generation and mast cell effector function in mice deficient in IL-4 and IL-13. Journal of Immunology, 174(12):7716-7724, 2005.

[419] Noelle R, Krammer PH, Ohara J, Uhr JW, and Vitetta ES. Increased expression of Ia antigens on resting B cells: an additional role for B-cell growth factor. Proceedings of the National Academy of Sciences of the United States of America, 81(19):6149-6153, 1984.

[420] Mohrs M, Holscher C, and Brombacher F. Interleukin-4 receptor alphadeficient BALB/c mice show an unimpaired T helper 2 polarization in response to Leishmania major infection. Infection and immunity, 68(4):1773-1780, 2000.

[421] Noben-Trauth N, Hu-Li J, and Paul WE. Conventional, naive CD4+ T cells provide an initial source of IL-4 during Th2 differentiation. Journal of Immunology, 165(7):3620-3625, 2000. 
[422] Zhu J, Min B, Hu-Li J, Watson CJ, Grinberg A, Wang Q, Killeen N, Urban JF, Guo L, and Paul WE. Conditional deletion of Gata3 shows its essential function in TH1-TH2 responses. Nature Immunology, 5(11):1157-1165, 2004.

[423] Zhu J, Cote-Sierra J, Guo L, and Paul WE. Stat5 activation plays a critical role in Th2 differentiation. Immunity, 19(5):739-748, 2003.

[424] Milner JD, Fazilleau N, McHeyzer-Williams M, and Paul W. Cutting Edge: Lack of High Affinity Competition for Peptide in Polyclonal CD4+ Responses Unmasks IL-4 Production. The Journal of Immunology, 184(12):6569 6573, 2010.

[425] Kaplan MH, Wurster AL, Smiley ST, and Grusby MJ. Stat6-dependent and -independent pathways for IL-4 production. Journal of Immunology, 163(12):6536-6540, 1999.

[426] Sokol CL, Barton GM, Farr AG, and Medzhitov R. A mechanism for the initiation of allergen-induced $\mathrm{T}$ helper type 2 responses. Nature Immunology, 9(3):310-318, 2007.

[427] Perrigoue JG, Saenz SA, Siracusa MC, Allenspach EJ, Taylor BC, Giacomin PR, Nair MG, Du Y, Zaph C, van Rooijen N, Comeau MR, Pearce EJ, Laufer TM, and Artis D. MHC class II-dependent basophilCD4+ T cell interactions promote TH2 cytokine-dependent immunity. Nature Publishing Group, 10(7):697-705, 2009.

[428] Giacomin PR, Siracusa MC, Walsh KP, Grencis RK, Kubo M, Comeau MR, and Artis D. Thymic Stromal Lymphopoietin-Dependent Basophils Promote Th2 Cytokine Responses following Intestinal Helminth Infection. The Journal of Immunology, 2012.

[429] Siracusa MC, Saenz SA, Hill DA, Kim BS, Headley MB, Doering TA, Wherry EJ, Jessup HK, Siegel LA, Kambayashi T, Dudek EC, Kubo M, Cianferoni A, Spergel JM, Ziegler SF, Comeau MR, and Artis D. TSLP promotes interleukin-3-independent basophil haematopoiesis and type 2 inflammation. Nature, 477(7363):229-233, 2011.

[430] Moro K, Yamada T, Tanabe M, Takeuchi T, Ikawa T, Kawamoto H, Furusawa JI, Ohtani M, Fujii H, and Koyasu S. Innate production of $\mathrm{T}(\mathrm{H}) 2$ cytokines by adipose tissue-associated c-Kit(+)Sca-1(+) lymphoid cells. Nature, 463(7280):540-544, 2010.

[431] Price AE, Liang HE, Sullivan BM, Reinhardt RL, Eisley CJ, Erle DJ, and Locksley RM. Systemically dispersed innate IL-13-expressing cells 
in type 2 immunity. Proceedings of the National Academy of Sciences of the United States of America, 107(25):11489-11494, 2010.

[432] Neill DR, Wong SH, Bellosi A, Flynn RJ, Daly M, Langford TKA, Bucks C, Kane CM, Fallon PG, Pannell R, Jolin HE, and McKenzie ANJ. Nuocytes represent a new innate effector leukocyte that mediates type-2 immunity. Nature, pp. 1-5, 2010.

[433] Wong SH, Walker JA, Jolin HE, Drynan LF, Hams E, Camelo A, Barlow JL, Neill DR, Panova V, Koch U, Radtke F, Hardman CS, Hwang YY, Fallon PG, and McKenzie ANJ. Transcription factor ROR is critical for nuocyte development. Nature Immunology, 13(3):229-236, 2012.

[434] Blázquez AB and Berin MC. Gastrointestinal dendritic cells promote Th2 skewing via OX40L. Journal of Immunology, 180(7):4441-4450, 2008.

[435] Flynn S, Toellner KM, Raykundalia C, Goodall M, and Lane P. CD4 $\mathrm{T}$ cell cytokine differentiation: the $\mathrm{B}$ cell activation molecule, OX40 ligand, instructs CD4 T cells to express interleukin 4 and upregulates expression of the chemokine receptor, Blr-1. The Journal of experimental medicine, 188(2):297304, 1998.

[436] Linton PJ, Bautista B, Biederman E, Bradley ES, Harbertson J, Kondrack RM, Padrick RC, and Bradley LM. Costimulation via OX40L Expressed by B Cells Is Sufficient to Determine the Extent of Primary CD4 Cell Expansion and Th2 Cytokine Secretion In Vivo. Journal of Experimental Medicine, 197(7):875-883, 2003.

[437] Vinuesa CG and Cyster JG. How T Cells Earn the Follicular Rite of Passage. Immunity, 35(5):671-680, 2011.

[438] Ronchese F and Hausmann B. B lymphocytes in vivo fail to prime naive $\mathrm{T}$ cells but can stimulate antigen-experienced $\mathrm{T}$ lymphocytes. The Journal of experimental medicine, 177(3):679-690, 1993.

[439] Deenick EK, Chan A, Ma CS, Gatto D, Schwartzberg PL, Brink R, and Tangye SG. Follicular Helper T Cell Differentiation Requires Continuous Antigen Presentation that Is Independent of Unique B Cell Signaling. Immunity, 33(2):241-253, 2010.

[440] Zotos D, Coquet JM, Zhang Y, Light A, D'Costa K, Kallies A, Corcoran LM, Godfrey DI, Toellner KM, Smyth MJ, Nutt SL, and Tarlinton DM. IL-21 regulates germinal center B cell differentiation and proliferation through a B cell-intrinsic mechanism. Journal of Experimental Medicine, 207(2):365-378, 2010. 
[441] King IL, Mohrs K, and Mohrs M. A Nonredundant Role for IL-21 Receptor Signaling in Plasma Cell Differentiation and Protective Type 2 Immunity against Gastrointestinal Helminth Infection. The Journal of Immunology, 185(10):6138-6145, 2010.

[442] Linterman MA, Beaton L, Yu D, Ramiscal RR, Srivastava M, Hogan JJ, Verma NK, Smyth MJ, Rigby RJ, and Vinuesa CG. IL-21 acts directly on B cells to regulate Bcl-6 expression and germinal center responses. Journal of Experimental Medicine, 207(2):353-363, 2010.

[443] Ozaki K. A Critical Role for IL-21 in Regulating Immunoglobulin Production. Science (New York, N.Y.), 298(5598):1630-1634, 2002.

[444] Talay O, Yan D, Brightbill HD, Straney EEM, Zhou M, Ladi E, Lee WP, Egen JG, Austin CD, Xu M, and Wu LC. IgE+ memory B cells and plasma cells generated through a germinal-center pathway. Nature Publishing Group, pp. 1-10, 2012.

[445] Yang Z, Sullivan BM, and Allen CDC. Fluorescent In Vivo Detection Reveals that IgE + B Cells Are Restrained by an Intrinsic Cell Fate Predisposition. Immunity, pp. 1-16, 2012.

[446] Snapper CM, Finkelman FD, and Paul WE. Differential regulation of IgG1 and IgE synthesis by interleukin 4. The Journal of experimental medicine, 167(1):183-196, 1988.

[447] Müller U, Stenzel W, Köhler G, Polte T, Blessing M, Mann A, Piehler D, Brombacher F, and Alber G. A GeneDosage Effect for Interleukin4 Receptor Chain Expression Has an Impact on Th2Mediated Allergic Inflammation during Bronchopulmonary Mycosis. The Journal of Infectious Diseases, 198(11):1714-1721, 2008.

[448] MacLennan ICM, Toellner KM, Cunningham AF, Serre K, Sze DMY, Zúñiga E, Cook MC, and Vinuesa CG. Extrafollicular antibody responses. Immunological Reviews, 194:8-18, 2003.

[449] Altin JA, Tian L, Liston A, Bertram EM, Goodnow CC, and Cook MC. Decreased T-cell receptor signaling through CARD11 differentially compromises forkhead box protein 3-positive regulatory versus TH2 effector cells to cause allergy. The Journal of allergy and clinical immunology, 127(5):12771285.e5, 2011.

[450] Kopf M, Brombacher F, Kohler G, Kienzle G, Widmann KH, Lefrang K, Humborg C, Ledermann B, and Solbach W. IL-4-deficient Balb/c mice 
resist infection with Leishmania major. The Journal of experimental medicine, 184(3):1127-1136, 1996.

[451] Huse M, Lillemeier BF, Kuhns MS, Chen DS, and Davis MM. T cells use two directionally distinct pathways for cytokine secretion. Nature Immunology, 7(3):247-255, 2006.

[452] Kupfer A, Mosmann TR, and Kupfer H. Polarized expression of cytokines in cell conjugates of helper T cells and splenic B cells. Proceedings of the National Academy of Sciences of the United States of America, 88(3):775-779, 1991.

[453] Asai K, Kitaura J, Kawakami Y, Yamagata N, Tsai M, Carbone DP, Liu FT, Galli SJ, and Kawakami T. Regulation of mast cell survival by IgE. Immunity, 14(6):791-800, 2001.

[454] Beyer K, Castro R, Birnbaum A, Benkov K, Pittman N, and Sampson HA. Human milk-specific mucosal lymphocytes of the gastrointestinal tract display a TH2 cytokine profile. Journal of Allergy and Clinical Immunology, 109(4):707-713, 2002.

[455] Neel NF, Creasy BM, Rankin JN, Pierce EM, McCoy ME, Daner RH, Fowler JA, Daniel JC, and Lantz CS. Absence of interleukin-3 does not affect the severity of local and systemic anaphylaxis but does enhance eosinophil infiltration in a mouse model of allergic peritonitis. Immunology letters, 95(1):37-44, 2004.

[456] Ganeshan K, Neilsen CV, Hadsaitong A, Schleimer RP, Luo X, and Bryce PJ. Impairing oral tolerance promotes allergy and anaphylaxis: A new murine food allergy model. The Journal of allergy and clinical immunology, 123(1):231-238.e4, 2009.

[457] Mizushima T, Ito T, Kishi D, Kai Y, Tamagawa H, Nezu R, Kiyono $\mathbf{H}$, and Matsuda $\mathbf{H}$. Therapeutic effects of a new lymphocyte homing reagent FTY720 in interleukin-10 gene-deficient mice with colitis. Inflammatory bowel diseases, 10(3):182-192, 2004.

[458] Adel-Patient K, Wavrin S, Bernard H, Meziti N, Ah-Leung S, and Wal JM. Oral tolerance and Treg cells are induced in BALB/c mice after gavage with bovine -lactoglobulin. Allergy, 66(10):1312-1321, 2011.

[459] Gavett SH, Chen X, Finkelman F, and Wills-Karp M. Depletion of murine CD4+ T lymphocytes prevents antigen-induced airway hyperreactivity and pulmonary eosinophilia. American journal of respiratory cell and molecular biology, 10(6):587-593, 1994. 
[460] Granato DA and Piguet PF. A mouse monoclonal IgE antibody anti bovine milk beta-lactoglobulin allows studies of allergy in the gastrointestinal tract. Clinical and experimental immunology, 63(3):703-710, 1986.

[461] Ohmori K, Luo Y, Jia Y, Nishida J, Wang Z, Bunting KD, Wang D, and Huang H. IL-3 Induces Basophil Expansion In Vivo by Directing Granulocyte-Monocyte Progenitors to Differentiate into Basophil LineageRestricted Progenitors in the Bone Marrow and by Increasing the Number of Basophil/Mast Cell Progenitors in the Spleen. The Journal of Immunology, 182(5):2835-2841, 2009.

[462] Madden KB, Urban JF, Ziltener HJ, Schrader JW, Finkelman FD, and Katona IM. Antibodies to IL-3 and IL-4 suppress helminth-induced intestinal mastocytosis. Journal of Immunology, 147(4):1387-1391, 1991.

[463] McDermott JR, Bartram RE, Knight PA, Miller HRP, Garrod DR, and Grencis RK. Mast cells disrupt epithelial barrier function during enteric nematode infection. Proceedings of the National Academy of Sciences of the United States of America, 100(13):7761-7766, 2003.

[464] Fitzpatrick DR and Kelso A. Dissociated expression of granulocytemacrophage CSF and IL-3 in short-term T cell clones from normal mice. Journal of Immunology, 155(11):5140-5150, 1995.

[465] Razin E, Ihle JN, Seldin D, Mencia-Huerta JM, Katz HR, LeBlanc PA, Hein A, Caulfield JP, Austen KF, and Stevens RL. Interleukin 3: A differentiation and growth factor for the mouse mast cell that contains chondroitin sulfate E proteoglycan. Journal of Immunology, 132(3):1479-1486, 1984 .

[466] Dvorak AM, Seder RA, Paul WE, Morgan ES, and Galli SJ. Effects of interleukin-3 with or without the c-kit ligand, stem cell factor, on the survival and cytoplasmic granule formation of mouse basophils and mast cells in vitro. AJPA, 144(1):160-170, 1994.

[467] Ruitenberg EJ and Elgersma A. Absence of intestinal mast cell response in congenitally athymic mice during Trichinella spiralis infection. Nature, 264(5583):258-260, 1976.

[468] Chen CC, Grimbaldeston MA, Tsai M, Weissman IL, and Galli SJ. Identification of mast cell progenitors in adult mice. Proceedings of the National Academy of Sciences of the United States of America, 102(32):11408-11413, 2005.

[469] Rodewald HR, Dessing M, Dvorak AM, and Galli SJ. Identification 
of a committed precursor for the mast cell lineage. Science (New York, N.Y.), 271(5250):818-822, 1996.

[470] Robertson JM, Jensen PE, and Evavold BD. DO11.10 and OT-II T cells recognize a C-terminal ovalbumin 323-339 epitope. Journal of Immunology, 164(9):4706-4712, 2000.

[471] Yamada A, Ohshima Y, Yasutomi M, Ogura K, Tokuriki S, Naiki H, and Mayumi M. Antigen-primed splenic CD8+ T cells impede the development of oral antigen-induced allergic diarrhea. Journal of Allergy and Clinical Immunology, 123(4):889-894, 2009.

[472] Huang C, Sali A, and Stevens RL. Regulation and function of mast cell proteases in inflammation. Journal of Clinical Immunology, 18(3):169-183, 1998.

[473] Crooks C, Ameratunga R, Brewerton M, Torok M, Buetow S, Brothers S, Wall C, and Jorgensen P. Adverse reactions to food in New Zealand children aged 0-5 years. New Zealand Medical Journal, 123(1327):14-23, 2009.

[474] Johansson A, Lugand D, Rolet-Répécaud O, Mollé D, Delage MM, Peltre G, Marchesseau S, Léonil J, and Dupont D. Epitope characterization of a supramolecular protein assembly with a collection of monoclonal antibodies: The case of casein micelle. Molecular Immunology, 46(6):1058-1066, 2009 .

[475] Henry G, Mollé D, Morgan F, Fauquant J, and Bouhallab S. HeatInduced Covalent Complex between Casein Micelles and -Lactoglobulin from Goat's Milk: Identification of an Involved Disulfide Bond. Journal of agricultural and food chemistry, 50(1):185-191, 2002.

[476] Cebo C, Lopez C, Henry C, Beauvallet C, Ménard O, Bevilacqua C, Bouvier F, Caillat $\mathbf{H}$, and Martin P. Goat s1-casein genotype affects milk fat globule physicochemical properties and the composition of the milk fat globule membrane. Journal of dairy science, 95(11):6215-6229, 2012.

[477] Forbes-Blom E, Camberis M, Prout M, Tang SC, and Gros G. Staphylococcal-derived superantigen enhances peanut induced Th2 responses in the skin. Clinical \& Experimental Allergy, 42(2):305-314, 2011.

[478] Eriksen EK, Holm H, Jensen E, Aaboe R, Devold TG, Jacobsen M, and Vegarud GE. Different digestion of caprine whey proteins by human and porcine gastrointestinal enzymes. British Journal of Nutrition, 104(03):374-381, 2010 .

[479] Hepworth MR, Daniłowicz-Luebert E, Rausch S, Metz M, Klotz C, 
Maurer M, and Hartmann S. Mast cells orchestrate type 2 immunity to helminths through regulation of tissue-derived cytokines. Proceedings of the National Academy of Sciences, 109(17):6644-6649, 2012.

[480] Aalberse RC. Assessment of allergen cross-reactivity. Clinical and molecular allergy : CMA, 5:2, 2007.

[481] Sudowe SS, Rademaekers AA, and Kölsch EE. Antigen dose-dependent predominance of either direct or sequential switch in $\operatorname{IgE}$ antibody responses. Immunology, 91(3):464-472, 1997.

[482] Frohlich A, Marsland BJ, Sonderegger I, Kurrer M, Hodge MR, Harris NL, and Kopf M. IL-21 receptor signaling is integral to the development of Th2 effector responses in vivo. Blood, 109(5):2023-2031, 2007.

[483] Li H, Ma Y, Xiang J, Xu G, Chen L, Cheng J, Wang J, Li Q, and He S. Comparison of the immunogenicity of yak milk and cow milk. European Food Research and Technology, 233(4):545-551, 2011. 


\section{Chapter 8}

\section{Appendices}

\subsection{Supporting data}

\subsubsection{References for figure 1.1}

IFinkelman, 2007. J Allergy Clin Immunol. 120:506-515.

2Jancar et al., 1991. 15:347-354.

${ }_{3}$ Strait et al., 2003. J Immunol. 170:3835-3842.

${ }_{4}$ Feyerabend et al., 2011. Immunity 35:1-13.

${ }_{5}$ Brown, 2009. Emergencias. 21:213-223.

${ }_{6}$ Scott et al., 1996. Canadian J Physiol Pharm. 74:320-330

${ }_{7}$ Scott \& Maric, 1993. Digestive Dis Sci. 38:394-402.

${ }_{8}$ Scott et al., 1990. Am J Physiol Gastrointest Liver Physiol. 259:G6-G14.

${ }_{9}$ Scott et al., 1988. Am J Physiol Gastrointest Liver Physiol. 255:G505-G511.

${ }_{10}$ Oliver et al., 1996. Neurogastroenterol Mot. 8:121-130.

${ }_{11}$ Oliver et al., 1995. Am J Physiol Gastrointest Liver Physiol. 268:G764-G771.

${ }_{12}$ Mourad et al., 1995. Gut. 36:553-557.

${ }_{13}$ Hanane et al., 2009. Am J Immunol. 5:56-60.

${ }_{14}$ Forbes et al., 2008. J Exp Med. 205:897-913.

${ }_{15}$ Brandt et al., 2003. J Clin Invest. 112:1666-1677.

${ }_{16}$ Sampson, 1999. J All Clin Immunol. 103:717-728.

${ }_{17}$ Arias et al., 2009. J All Clin Immunol.124:307-314.

Figure 8.1: References for figure 1.1. These references relate specifically to figure 1.1 in the introduction section. 


\subsubsection{Genotyping of IL-3 and IL-9 transgene}

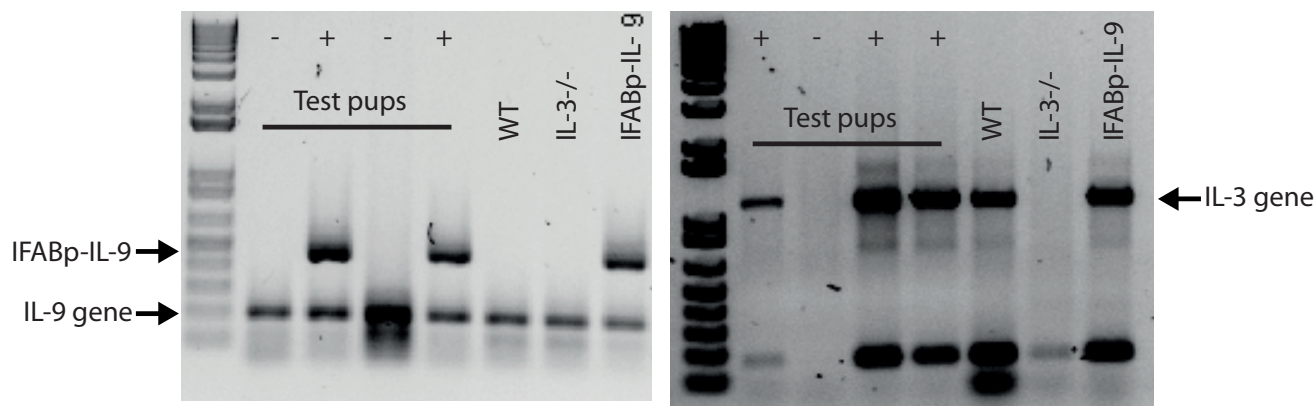

Figure 8.2: Genotype testing of IL-3 and IL-9. (A) Mice were tested for the presence of the wild-type IL-9 and the IL-9 transgene. (B) Mice were tested for the presence of the wild-type IL-3 gene. Primer pairs can be found in the methods section. 
8.1.3 Flow Cytometry reagents 


\begin{tabular}{|c|c|c|c|}
\hline Specificity & Fluorophore & Clone & Source \\
\hline \multirow[t]{2}{*}{ B220 } & Horizon V450 & Ra3-6B2 & BD Pharmingen \\
\hline & PE-Texas Red & Ra3-6B2 & BD Pharmingen \\
\hline CD3 & Alexafluor $\AA 488$ & $500 \mathrm{~A} 2$ & Invitrogen Molecular Probes \\
\hline \multirow[t]{6}{*}{ CD4 } & Allophycocyanin (APC) & RM4-5 & BD Pharmingen \\
\hline & $\mathrm{APC} / \mathrm{H} 7$ & GK1.5 & BD Pharmingen \\
\hline & Fluorescein Isothiocyanate (FITC) & GK1.5 & In house \\
\hline & Phycoerythrin (PE) & GK1.5 & BD Pharmingen \\
\hline & Peridinin Chlorophyll Protein (Percp) & RM4-5 & BD Pharmingen \\
\hline & Quantum dot605® & RM4-5 & invitrogen Molecular Probes \\
\hline \multirow[t]{2}{*}{ CD8a } & Alexafluor $\AA 700$ & 536.7 & Biolegend \\
\hline & $\mathrm{PE}-\mathrm{Cy} 7$ & $53-6.7$ & BD Pharmingen \\
\hline CD19 & APC-H7 & ID3 & BD Pharmingen \\
\hline CD44 & $\mathrm{PE}$ & IM7 & BD Pharmingen \\
\hline CD45 & Pacific Blue $\AA$ & $30 \mathrm{~F} 11$ & Biolegend \\
\hline \multirow[t]{2}{*}{ CD62L } & Biotin & MEL-14 & BD Pharmingen \\
\hline & Pacific Blue $\AA$ & MEL-14 & Biolegend \\
\hline CD117) & APC & $2 \mathrm{~B} 8$ & BD Pharmingen \\
\hline FceRI & $\mathrm{PE}$ & Mar-1 & eBioscience \\
\hline CD16/32 & FITC & $2.4 \mathrm{G} 2$ & BD Pharmingen \\
\hline human CD2 & $\mathrm{APC}$ & RPA2.10 & BD Pharmingen \\
\hline I-A/I-E & PE-Cy7 & M51114.15.2 & Biolegend \\
\hline IL-3 & $\mathrm{PE}$ & MP2-8F8 & BD Pharmingen \\
\hline \multirow[t]{2}{*}{ IL-4 } & APC & 11B11 & BD Pharmingen \\
\hline & $\mathrm{PE}$ & 11B11 & BD Pharmingen \\
\hline IL-5 & $\mathrm{PE}$ & TRFK-5 & BD Pharmingen \\
\hline IL-10 & $\mathrm{PE}$ & JES5-16E3 & BD Pharmingen \\
\hline IL-13 & $\mathrm{PE}$ & eBio13A & eBioscience \\
\hline $\operatorname{IgD}$ & $\mathrm{PE}$ & $11-26 c .2 \mathrm{a}$ & BD Pharmingen \\
\hline $\operatorname{IgE}$ & biotin & R35-118 & BD Pharmingen \\
\hline IgG1 & biotin & A85.1 & BD Pharmingen \\
\hline CD49b & PE-Cy7 & DX5 & eBioscience \\
\hline \multirow[t]{8}{*}{ Biotin } & $\mathrm{APC}$ & Streptavidin & BD Pharmingen \\
\hline & FITC & Streptavidin & BD Pharmingen \\
\hline & $\mathrm{PE}$ & Streptavidin & BD Pharmingen \\
\hline & PE-Texas Red® & Streptavidin & BD Pharmingen \\
\hline & 7-Amino Actinomycin D (7-AAD) & & BD Pharmingen \\
\hline & 4,6-diaminidino-2-phenylindole (DAPI) & & Invitrogen Molecular Probes \\
\hline & Live/Dead@ fixable blue & & Invitrogen Molecular Probes \\
\hline & Golgistop (with Monensin) & & BD Pharmingen \\
\hline
\end{tabular}


8.1.4 Flow cytometers 


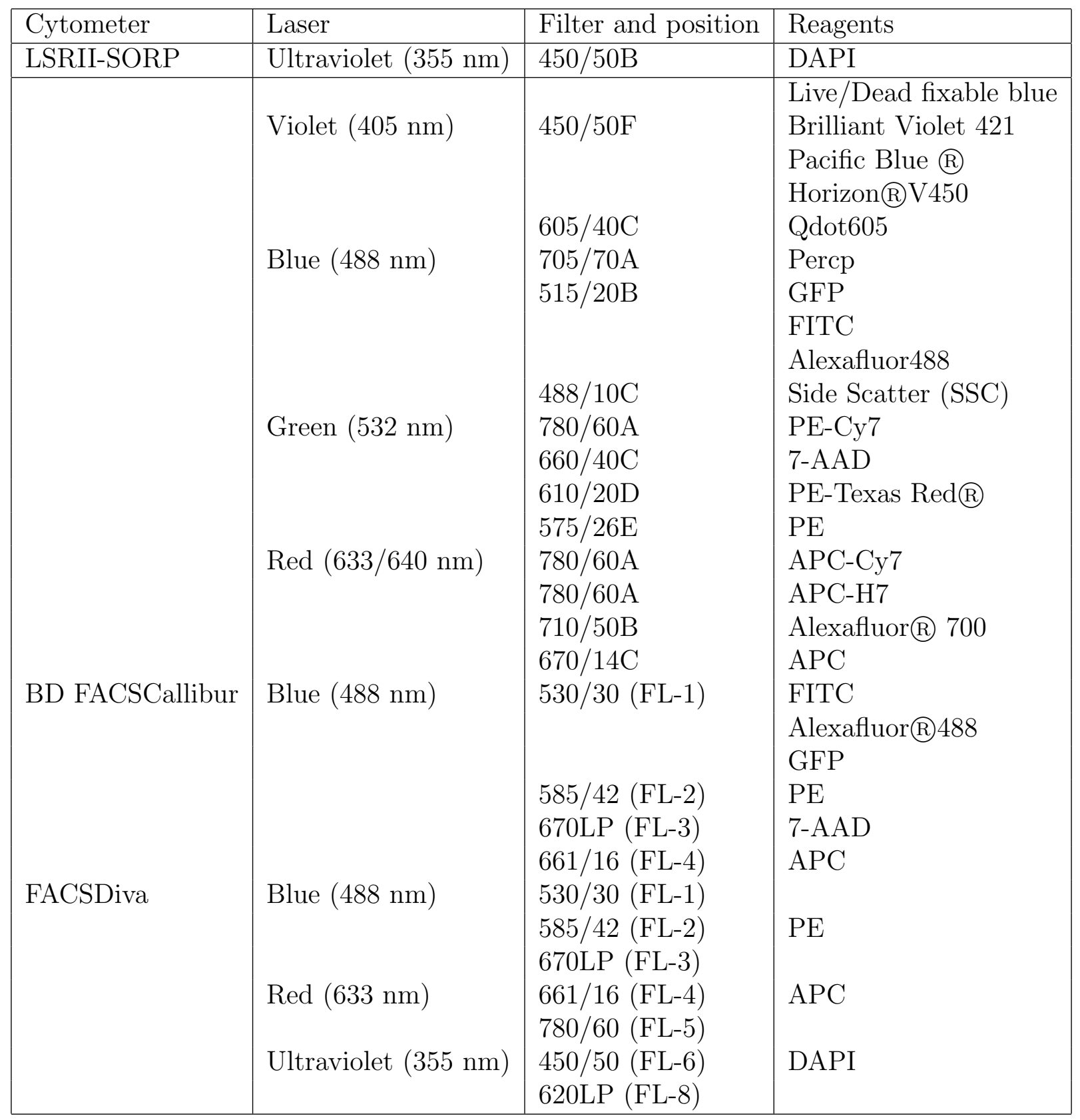




\subsubsection{Supplementary data}
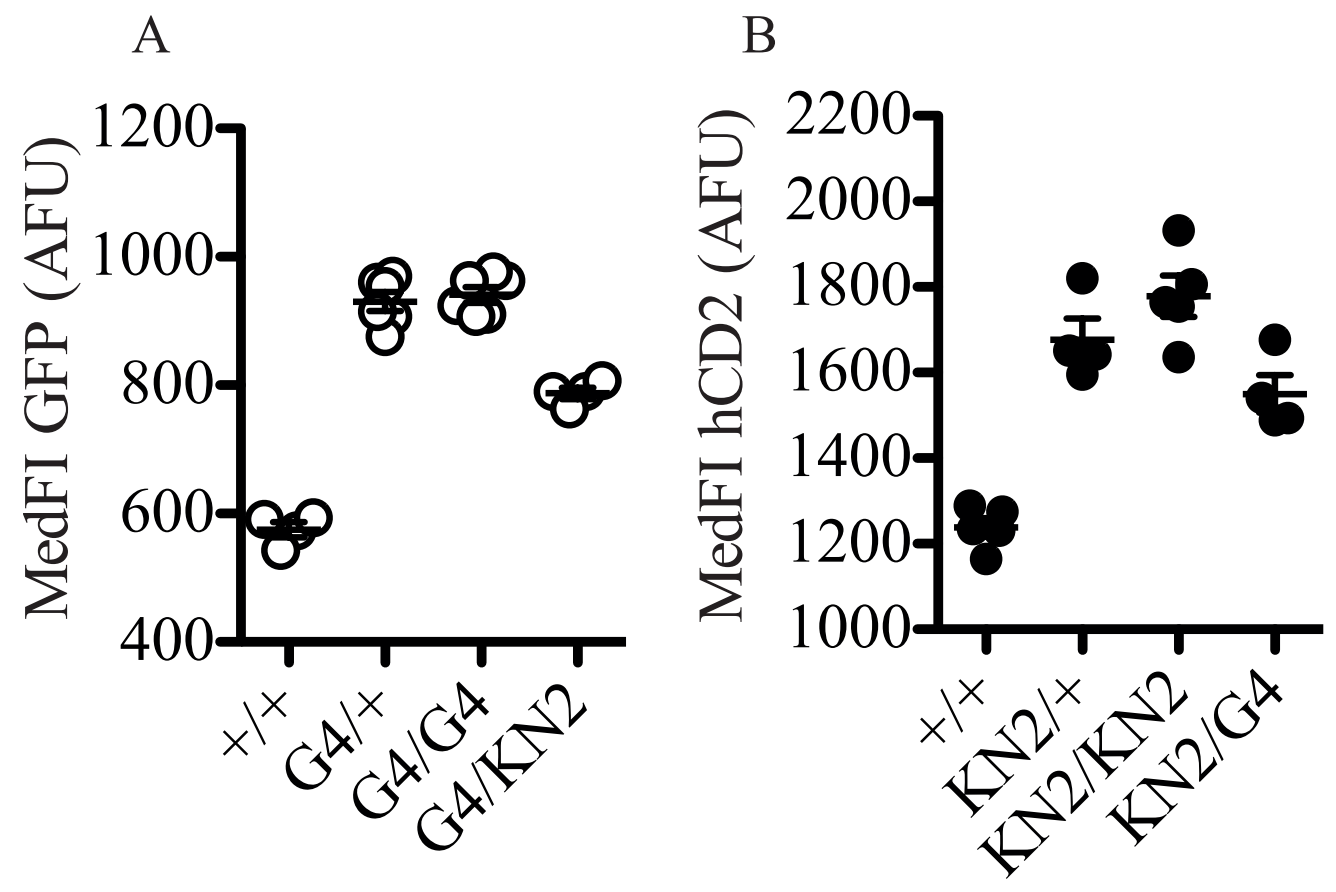

Figure 8.3: GFP and hCD2 medFI are lower in KN2/G4 cells than G4/+, $\mathrm{KN} 2 /+$, G4/G4 and KN2/KN2 cells. Ten days after intradermal immunization with $100 \mu \mathrm{g}$ HDM, auricular lymph nodes were excised. (A) The GFP median fluorescence intensity of total GFP + CD4+ cells was examined. (B) The hCD2 median fluorescence intensity of total hCD2+ CD4+ cells was examined. Data are shown from one of the experiments used to compile data in figures 4.2 and 4.3. AFU=Arbitrary Fluorescence Units. 

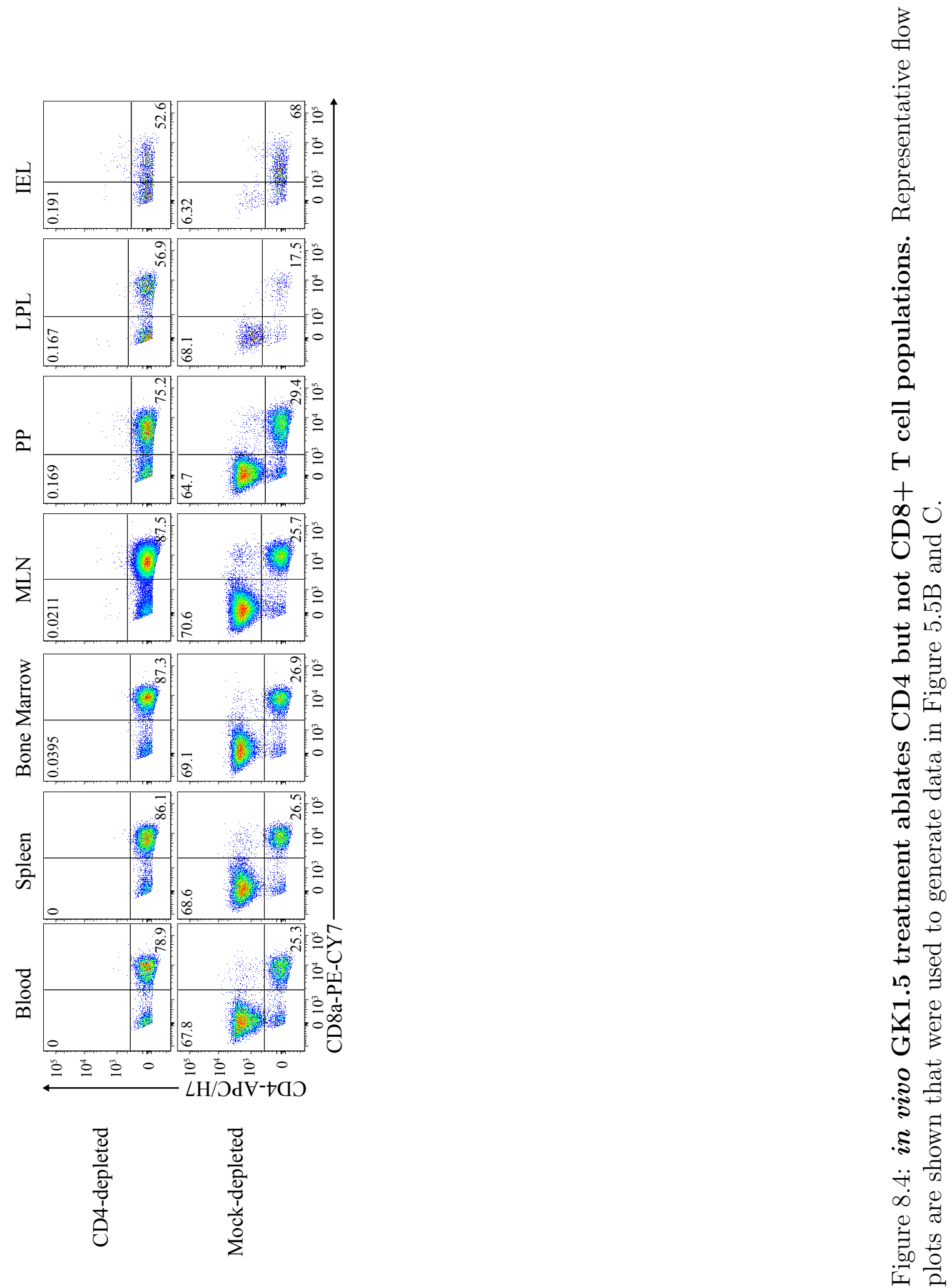

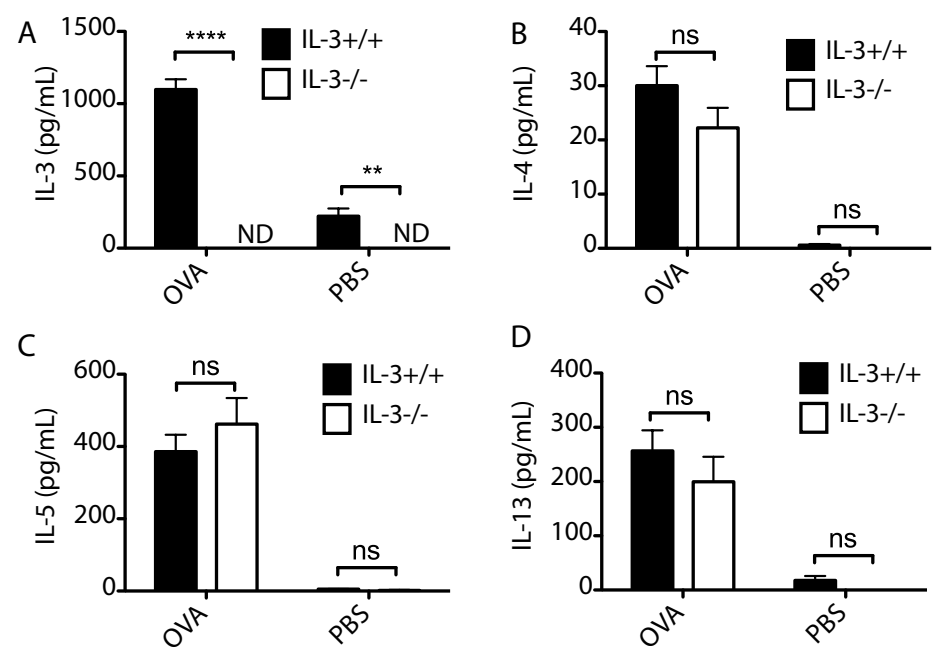

Figure 8.5: Cytokine production by OVA or PBS-stimulated splenocytes from IL-3 sufficient and deficient mice. Mice were sensitized to OVA plus alum twice by ip injection and then were mock-challenged with PBS 10 times. Spleens were excised and cultured with OVA (OVA) or medium alone (PBS). After four days supernatants were collected and examined for (A) IL-3, (B) IL-4, (C) IL-5 and (D) IL-13 production using a bioplex assay. Data bars show mean + SEM of individually cultured spleens ( $\mathrm{n}=5$ /group). ${ }^{* *} \mathrm{p}<0.01,{ }^{* * * *} \mathrm{p}<0.0001$ by Two-way ANOVA with Bonferroni's post-test. 

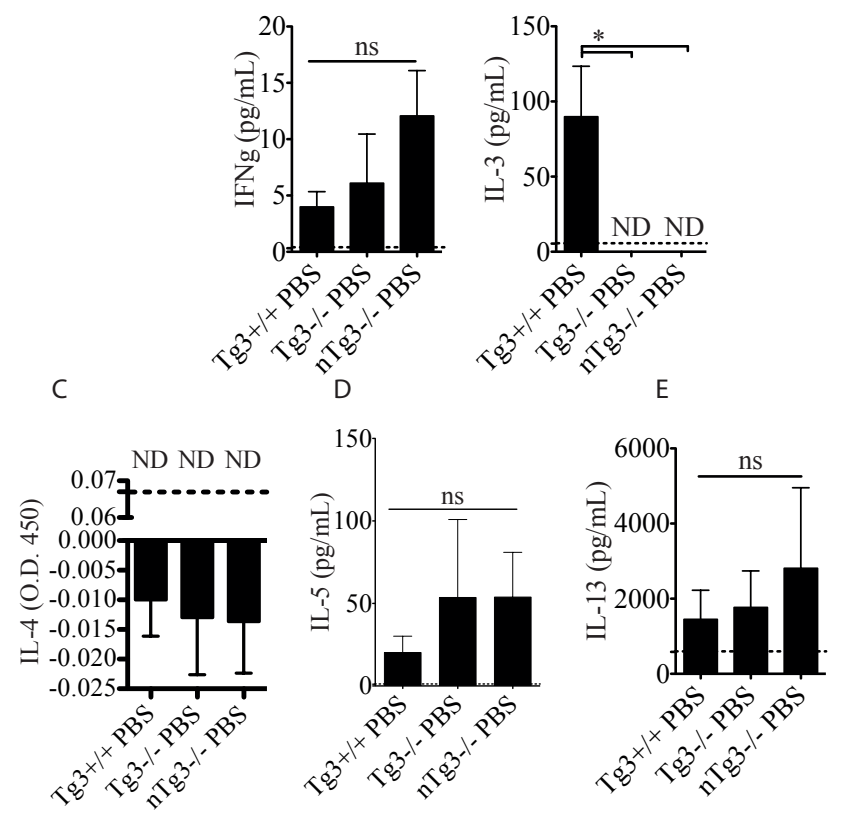

Figure 8.6: Cytokine production by OVA or PBS-stimulated mesenteric lymph node cells from $\mathbf{T g}+/+, \mathrm{Tg}-/-$ and $\mathbf{n T g}-/-$ mice. Mice were sensitized to OVA plus alum twice by ip injection and then were mock-challenged with PBS 11 times. Mesenteric lymph nodes were $28 x$ cised and cells were cultured with OVA. After three days supernatants were collected and examined for (A) IFN- $\gamma$, (B) IL-3, (C) IL-4 and (D) IL-5 and (E) IL-13 production by ELISA. Data bars show mean + SEM of individually cultured MLNs ( $\mathrm{n}=3$ /group). Data were compared by One-way ANOVA with Tukey's post-test. Dashed lines represent the assay limit. ND, none detected. 


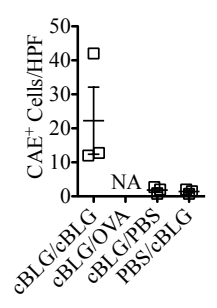

Figure 8.7: Mastocytosis in caprine BLG sensitized mice. Jejunal mast cell frequencies were determined by CAE staining at the experimental endpoint in mice from Figure 6.8. NA not analysed. 


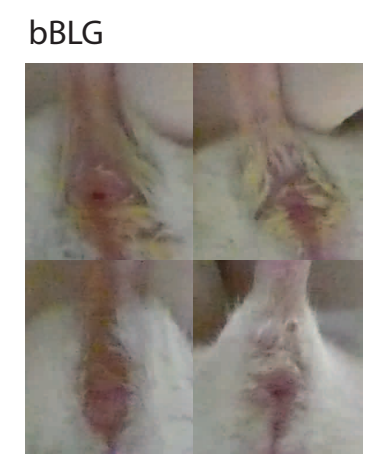

BSA

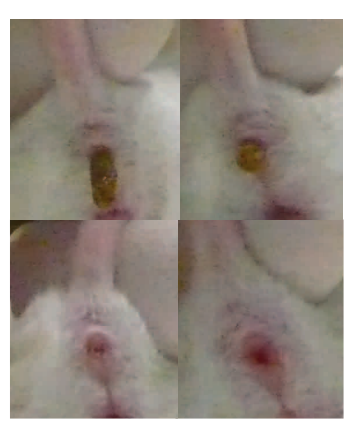

CBLG

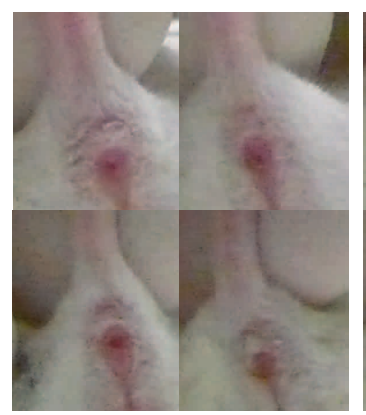

PBS

OVA
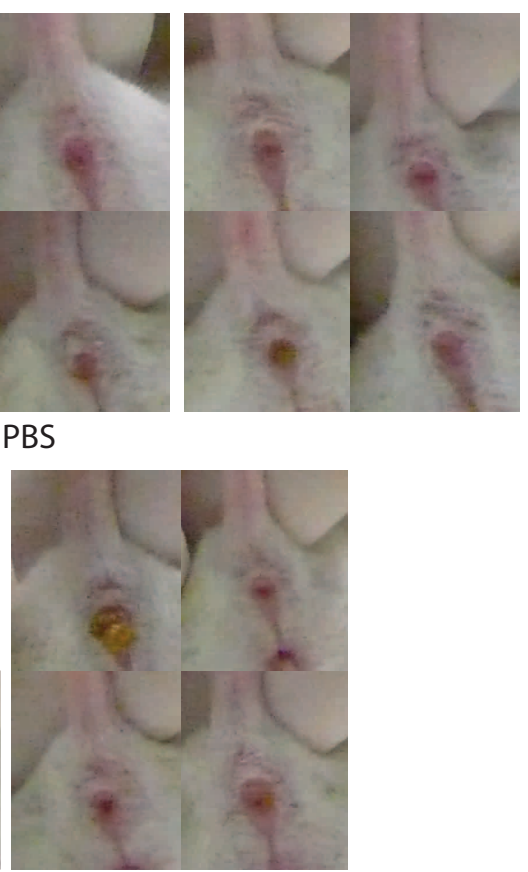

Figure 8.8: Liquid stool at 60 minutes post-challenge three in intestinally atopic mice fed different allergens. 60 minutes after the third oral gavage with sensitizing antigen (day 13), iIFABP p-IL-9 mice were photographed. Diarrhoea was not observed in any mice in a second experiment in which sensitized or naive mice were fed three times with $50 \mathrm{mg}$ cow BLG. 